\title{
GEORG-AUGUST-UNIVERSITÄT
} GÖTTINGEN

\section{Microlocal Analysis of Tempered Distributions}

\author{
DisSERTATION \\ Zur Erlangung des
}

mathematisch-naturwissenschaftlichen Doktorgrades

"Doctor rerum naturalium"

im Promotionsprogramm "School of Mathematical Sciences"

der Georg-August University School of Science (GAUSS)

vorgelegt von

RenÉ M. SCHulz

aus Ingolstadt

Göttingen, 2014 


\section{Betreunngsausschuss}

Erstbetreuerin: Prof. Dr. Dorothea Bahns

Mathematisches Institut

Georg-August-Universität Göttingen

Zweitbetreuer: Prof. Dr. Ingo Witt

Mathematisches Institut

Georg-August-Universität Göttingen

Mitglieder DeR PRÜFungskommission

Referentin: Prof. Dr. Dorothea Bahns

Mathematisches Institut

Georg-August-Universität Göttingen

Ko-Referent: Prof. Dr. Ingo Witt

Mathematisches Institut

Georg-August-Universität Göttingen

Weitere Mitglieder DeR PrÜFungskommission

Prof. Dr. Gert Lube

Institut für Numerische und Angewandte Mathematik

Georg-August-Universität Göttingen

apl. Prof. Dr. Karl-Henning Rehren

Institut für Theoretische Physik

Georg-August-Universität Göttingen

Prof. Dr. Thomas Schick

Mathematisches Institut

Georg-August-Universität Göttingen

Prof. Dr. Max Wardetzky

Institut für Numerische und Angewandte Mathematik

Georg-August-Universität Göttingen

TAG Der mündlichen Prüfung: 12. September 2014 


\title{
MICROLOCAL ANALYSIS OF TEMPERED DISTRIBUTIONS
}

\author{
RENÉ M. SCHULZ
}

\begin{abstract}
In this dissertation we study tempered distributions from the microlocal point of view. The fundamental notion of microlocal analysis, the wave front set, is replaced by two analogues, the SG-wave front set and the G-wave front set associated to the global pseudo-differential calculi with SG- and Shubin symbols respectively. Properties of these global wave front sets are collected and we establish various ways to characterize them, in particular in terms of the FBI-transform.

We generalize constructions that involve the classical wave front set to the global setting, in particular operations on tempered distributions, such as pull-backs, (twisted) products and pairings, for which we give microlocal existence criteria.

As an application, we introduce a class of tempered oscillatory integrals, parametrized by inhomogeneous phase functions and amplitudes from SG-symbol spaces. We study the SG-wave front set of such distributions, which turns out to be bounded by a generalization of the notion of stationary phase points.

In this framework, we establish the notion of SG-Lagrangian, which generalizes the classical notion of conic Lagrangian submanifolds of $T^{*} \mathbb{R}^{d}$. In particular, we study parametrization properties of these objects and it turns out that locally, each such SG-Lagrangian is realized as the stationary points of a SG-phase function.

As further applications, we revisit certain constructions involving distributions from axiomatic quantum field theory and show how these may be realized in the tempered setting.
\end{abstract}

2010 Mathematics Subject Classification. 35A18, 35S05, 35S30, 46F10, 46F12, 53D12, $81 \mathrm{~T} 15$. Key words and phrases. Convolution, extensions of distributions, FBI transform, Gelfand-Shilov spaces, Lagrangian, microlocal analysis, oscillatory integral, product, Pseudo-differential operator, scaling degree, short-time Fourier transform, tempered distribution, time frequency analysis, two-point function, wave front set, Weyl product. 


\section{ZUSAMMENFASSUNG}

Diese Dissertation ist dem Studium temperierter Distributionen mittels mikrolokaler Methoden gewidmet. Die fundamentale Größe der mikrolokalen Analysis, die Wellenfrontmenge, wird durch zwei analoge Konzepte ersetzt, die den pseudodifferentiellen SG- und Shubin-Kalkülen zugeordnet sind. Die Eigenschaften dieser globalen Wellenfrontmengen werden studiert und ferner werden unterschiedliche Möglichkeiten, diese globalen Singularitäten zu charakterisieren, untersucht, insbesondere mittels der FBI-Transformation.

Zahlreiche Konstruktionen, die den klassischen Wellenfrontmengenbegriff beinhalten, werden in den globalen Kontext übersetzt, insbesondere Rechenoperationen mit temperierten Distributionen wie etwa (getwistete) Produkte, Pull-backs und Paarungen, für die mikrolokale Existenzkriterien angegeben werden.

Als eine Anwendung wird eine Klasse von temperierten Oszillatorintegralen eingeführt, welche durch inhomogene Phasenfunktionen und Amplituden aus SGSymbolklassen parametrisiert werden. Die SG-Wellenfrontmengen dieser Distributionen werden untersucht und es stellt sich heraus, dass diese durch eine Verallgemeinerung der Menge stationärer Punkte der Phasenfunktionen beschränkt werden.

In diesem Kontext wird eine Verallgemeinerung des klassischen Begriffs einer konischen Lagrange-Untermannifaltigkeit des $T^{*} \mathbb{R}^{d}$ vorgenommen und diese Objekte werden auf ihre Parametrisierungseigenschaften untersucht. Es stellt sich heraus, dass jedes solche Objekt lokal als die Menge der stationären Punkte einer SGPhasenfunktion realisiert werden kann.

Als weitere Anwendung werden einige Konstruktionen der axiomatischen Quantenfeldtheorie, die Distributionen beinhalten, im temperierten Kontext realisiert. 


\section{Contents}

Introduction 1

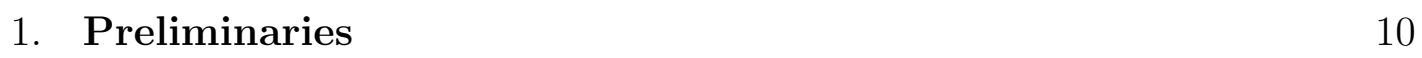

1.1. Compactification of Euclidean space 10

\begin{tabular}{lll}
\hline 1.1.1. Radial compactification of Euclidean space & 10
\end{tabular}

$\begin{array}{lll}1.1 .2 . & \text { Cut-offs and cut-offs "at infinity" } & 14\end{array}$

$\begin{array}{lll}\text { 1.1.3. Singularities at infinity } & 18\end{array}$

\begin{tabular}{lll}
\hline 1.1 .4 . Two ways to compactify the cotangent space & 19
\end{tabular}

1.2. Global symbol classes on Euclidean spaces 22

1.2.1. Introduction and symbols in one set of variables 22

$\begin{array}{lll}1.2 .2 \text {. The space of Shubin G-symbols } 24 & 24\end{array}$

$\begin{array}{lll}1.2 .3 . & \text { The space of SG-symbols } & 25\end{array}$

$\begin{array}{lll}\text { 1.2.4. Classical SG-symbols } & 27\end{array}$

2. Microlocal singularities and operations 32

$\begin{array}{lll}2.1 . & \text { Introduction } & 32\end{array}$

2.2. The SG-wave front set 35

$\begin{array}{lll}2.2 .1 \text {. Introduction, basic properties and some first examples } & 35\end{array}$

$\begin{array}{lll}2.2 .2 . & \text { Existence of distributions with assigned singularities } & 39\end{array}$

$\begin{array}{lll}2.3 \text {. Characterization of } \mathrm{WF}_{\mathrm{SG}} \text { in the time-frequency plane } 46 & 46\end{array}$

2.3.1. Characterization of $\mathrm{WF}_{\mathrm{SG}}^{\psi}$ and $\mathrm{WF}_{\mathrm{SG}}^{e}$ in terms of FBI transforms 46

2.3.2. Characterization of the corner component 48

2.4. Operations on tempered distributions using $\mathrm{WF}_{\mathrm{SG}} \quad 53$

$\begin{array}{lll}2.4 .1 . \quad \text { (Multi-)linear operations } & 53\end{array}$

$\begin{array}{lll}2.4 .2 . & \text { Composition with operators } & 61\end{array}$

$\begin{array}{lll}2.4 .3 . \quad \text { Affine maps and translation invariant distributions } & 62\end{array}$

2.5. The G-wave front set 65

2.5.1. Introduction, basic properties and some first examples 65

2.6. Characterization of $\mathrm{WF}_{\mathrm{G}}$ in the time-frequency plane 68

2.6.1. Characterization in terms of the STFT 68

$\begin{array}{lll}2.6 .2 . & \text { G-microlocality of operators } & 71\end{array}$

$\begin{array}{lll}2.6 .3 . & \text { Existence of distributions with assigned singularities } & 72\end{array}$

$\begin{array}{lll}2.6 .4 . & \text { A semi-classical description } & 76\end{array}$

2.6.5. A comparison between $\mathrm{WF}_{\mathrm{SG}}$ and $\mathrm{WF}_{\mathrm{G}}$. 81

2.7. Operations on tempered distributions using $\mathrm{WF}_{\mathrm{G}} \quad 84$ 
2.7.1. Multilinear operations and composition with operators 84

$2.7 .2 . \quad$ Affine maps 86

$\begin{array}{lll}3 . & \text { Special constructions and applications } & 88\end{array}$

3.1. Outline 88

3.2. Tempered oscillatory integrals 90

3.2.1. Introduction and elements of the classical theory 90

3.2.2. Definition of tempered oscillatory integrals 93

$\begin{array}{lll}3.2 .3 . & \text { Singularities of tempered oscillatory integrals } & 98\end{array}$

$\begin{array}{lll}3.2 .4 . \quad \text { A study of the underlying geometry } & 104\end{array}$

3.3. Scaling properties of tempered distributions 123

3.3.1. Weinstein's order and symbol of a (tempered) distribution 123

$\begin{array}{lll}3.3 .2 \text {. The Steinmann scaling degree and extensions of distributions } & 127\end{array}$

3.4. Weyl-quantized operators and the Weyl product 139

$\begin{array}{lll}3.4 .1 . & \text { Weyl products of tempered distributions } & 139\end{array}$

3.4.2. Weyl-quantization of tempered distributions 141

$\begin{array}{lll}3.5 . & \text { Applications to quantum field theory } & 144\end{array}$

3.5.1. Introduction 144

3.5.2. The two-point function as a tempered oscillatory integral $\quad 145$

3.5.3. Well-definedness of distributional products in QFT 150

4. Outlook 155

$\begin{array}{lll}4.1 . \quad \text { Microlocal analysis on Gelfand-Shilov spaces } & 155\end{array}$

4.2. General outlook 161

Appendix A. Collection of auxiliary results 166

$\begin{array}{lll}\text { A.1. } & \text { Notation, spaces of test functions and distributions } & 166\end{array}$

\begin{tabular}{lll}
\hline A.2. Schwartz functions and tempered distributions & 167
\end{tabular}

$\begin{array}{lll}\text { A.2.1. Basics of time-frequency analysis on tempered distributions } & 169\end{array}$

$\begin{array}{lll}\text { A.3. Elements of pseudodifferential calculus } & 173\end{array}$

$\begin{array}{lll}\text { A.3.1. Pseudodifferential operators and parametrices } & 173\end{array}$

\begin{tabular}{lll}
\hline A.3.2. Sobolev spaces and $L^{2}$-boundedness & 177
\end{tabular}

A.3.3. The action of pseudo-differential operators and the STFT 181

$\begin{array}{ll}\text { Appendix B. List of recurring symbols and abbreviations } & 183\end{array}$

$\begin{array}{ll}\text { References } & 186\end{array}$ 
$\begin{array}{lll}1 & \text { Radial compactification of } \mathbb{R}^{d} & 11\end{array}$

$\begin{array}{lll}2 & \text { A model cut-off at infinity } & 15\end{array}$

\begin{tabular}{|lll}
\hline 3 & Construction of an asymptotic cut-off by summation and dilation & 17
\end{tabular}

4 Construction of a function $a_{M=1}^{+\infty}$ for $\lambda=2, N=1 \quad 18$

5 Two compactifications of $T^{*} \mathbb{R}$ with "neighbourhoods at infinity" 21

6 The construction of $u^{\psi}$, schematic 41

7 The construction of $u^{\psi e}$, schematic 43

8 The graph of $v(\cdot ; 1,1)($ up to $k=4)$

\begin{tabular}{|ll|l|}
\hline 9 & The STFTs of the distributions in Example & 2.59 \\
\hline
\end{tabular}

10 The graph of $\left|\mathscr{V}_{\psi_{0}} v(\cdot ; 1,1)\right|($ up to $k=4)$

11 Inclusion results of $\mathrm{WF}_{\mathrm{SG}}$ and $\mathrm{WF}_{\mathrm{G}}$. 82

12 Intersection of $\tilde{\Lambda}_{\varphi}^{\psi} \subset \widetilde{\mathrm{W}}_{\mathrm{SG}}^{\psi}$ and $\tilde{\Lambda}_{\varphi}^{e} \subset \widetilde{\mathrm{W}}_{\mathrm{SG}}^{e}$ at the corner $\widetilde{\mathrm{W}}_{\mathrm{SG}}^{\psi e} \quad 111$

13 The counterexample of Weinstein, schematic 124

14 Schematic comparison between extensions by scaling 138

\begin{tabular}{|ll}
\hline 15 The cone singular support of the two-point function & 149
\end{tabular}

$16 \widetilde{\mathrm{WF}}_{\mathrm{SG}}^{\psi}\left(\Delta_{+}\right) \cup \widetilde{\mathrm{WF}}_{\mathrm{SG}}^{\psi e}\left(\Delta_{+}\right)$and $\widetilde{\mathrm{WF}}_{\mathrm{SG}}^{\psi}\left(\mathcal{F} \Delta_{+}\right) \quad 150$

17 A bound for $\widetilde{\mathrm{WF}}_{\mathrm{SG}}^{\psi}\left(\Delta_{+}^{k}\right) \cup \widetilde{\mathrm{WF}}_{\mathrm{SG}}^{\psi e}\left(\Delta_{+}^{k}\right) \quad 152$

18 The short-time Fourier transform: localization by a window function $\begin{array}{ll}\text { (schematic) } & 171\end{array}$ 


\section{Introduction}

„Je weiter eine mathematische Theorie ausgebildet wird, desto harmonischer und einheitlicher gestaltet sich ihr Aufbau und ungeahnte Beziehungen zwischen bisher getrennten Wissenszweigen werden entdeckt. So kommt es, daß mit der Ausdehnung der Mathematik ihr einheitlicher Charakter nicht verloren geht, sondern desto deutlicher offenbar wird."

- David Hilbert 72$]$

\section{General Introduction}

This thesis treats the topic of microlocal analysis of tempered distributions. In the following, we will give a broad exposition of the topic and of our approach. We refer to the individual sections for more specific introductions for the topics treated therein.

Microlocal analysis is a tool to study (ultra-)distributions on some manifold $X$ in terms of a resolution of their singularities in the cotangent space $T^{*} X$. It has many applications in the theory of partial differential equations and spectral theory, as well as in other fields of study, such as constructive quantum field theory. The term "local" stands for localization by some means in the underlying space $X$ and the "micro" refers to the taking-into-account of the behaviour the covariable, or frequency, such as via the application of Fourier transformation. We give a brief historic exposition of the history of this notion, with reference to the historic notes in [81, 83] and [95].

The first application of the concept of microlocalization was in the framework of analytic singularities of hyperfunctions (i.e. analytic functionals) see Sato [124, 125], as well as Sato, Kawai and Kashiwara [126], making thorough use of methods from complex analysis and sheaf theory. In a different approach, Bros and Iagolnitzer [15] introduced an integral transform, the FBI-transform and studied analytic singularities in terms of decay with respect to a scaling parameter. Finally, Hörmander introduced a notion of wave front set (which we denote by $\mathrm{WF}_{\mathrm{cl}}(u)$ ) suitable for the microlocal study of $\mathscr{C}^{\infty}$-singularities of Schwartz distributions $u$ by pseudo-differential methods in [77], consider also [78, 779, 81, 83], which also has an analytic counterpart, see [81, Sect. 9.3.]. In particular he used these techniques to study the class of Lagrangian distributions, which arise as kernels of Fourier Integral operators and fundamental solutions of partial differential equations, consider also [56]. 
The beauty of microlocal analysis is that it provides a clear geometric picture for certain constructions involving distributions. In particular it is possible

- to prove propagation results (with emphasis on how singularities propagate along bicharacteristics, which yields a geometric interpretation in the spirit of Huygens),

- to prove existence and uniqueness results for solutions to partial differential equations,

- to generalize, whenever certain microlocal existence criteria are fulfilled, operations such as pull-backs by smooth maps and multiplication to distributions,

- to establish a calculus of Lagrangian distributions and Fourier integral operators, based on geometric assumptions.

In this thesis, we will mainly adopt the $\mathscr{C}^{\infty}$-approach, and we will define our objects of study in the spirit of Hörmander [79, 81, 82, 83]. In particular, we study singularities of tempered distributions in terms of generalizations and analogues to Hörmander's classical wave front set. Our main focus will be on how to generalize constructions from the local theory to the global one, which we will motivate in the following paragraph.

By now, microlocal analysis provides a well-established tool to analyse singularities of distributions in various contexts. The original framework in which these were introduced is local, meaning the classical notions are particularly suited to study $\mathscr{C}^{\infty}$-singularities at finite distances, that is the derivation of a distribution $u$ from being locally $\mathscr{C}^{\infty}$, and most constructions are suitable to treat problems on (small) open subsets of $\mathbb{R}^{d}$ and compact manifolds $X$.

In order to generalize these concepts to non-compact spaces, we need to make several adaptations.

- By introducing pseudo-differential calculi suitable for the analysis of tempered distributions, consider [9, 114, 135] and in particular the HörmanderWeyl calculus [83, 80, 94], it is possible to carry over the ideas of microlocal analysis to the global setting and to control also "growth" of distributions, such as with respect to weighted Sobolev spaces. We are going to Shubin G-calculus [135] as well as the SG-calculus introduced by Parenti [114], studied by many authors such as [44, 45, 46, 157, 131], and also under the name of sc-calculus, see e.g. [102, 107]. 
- In order to generalize microlocalization, it is important to impose a notion of "localization at infinity". We are going to focus on the case where $X=\mathbb{R}^{d}$. Even in this case, there are several reasonable choices for a microlocal structure "at infinity", and we are going to treat in detail two notions suitable for the two calculi used. For an efficient formulation of this underlying geometry, we are going to adopt the train of thoughts in [36, 57, 102] to consider suitable compactifications of $T^{*} X$, which amounts to equipping $X$ with an "asymptotically flat" structure, see also [128.

- Another development in microlocal analysis is the employment of methods of harmonic analysis, time-frequency analysis and semi-classical analysis, see e.g. [35, 40, 41, 51, 64, 94, 99, 157]. These methods often involve integral transforms that yield representations of a given distribution in the time-frequency plane and encode the behaviour in both the variable as well as the covariable. When the range of such transforms restricted to smooth functions is known, it is possible to use them to characterize smoothness. Using suitable means of localization, it is then clear that this may be used to characterize the wave front set in several ways.

In the global setting, these ideas provide various means to study global singularities, by combining (localizable) means to characterize smoothness with one of the many ways to study decay properties.

We recall that in [12], Bony has established that in the analytic setting the previously introduced "local" notions of analytic wave front set coincide, indeed all such notions admitting certain reasonable properties one would expect of an analytic wave front. In the global setting, however, there are several reasonable notions one might impose, depending on the "rate of oscillation" one is interested in, consider e.g. [102, 153]. One of the reasons to do so is the fact that the classical wave front set does not propagate in a "conventional fashion" under evolution of the Schrödinger equation, whereas singularities caused by "quadratic oscillations" indeed propagate nicely, as was shown in [111, 153].

We mention some of the several notions of wave front set suitable for the treatment of global singularities, such as the $\mathscr{S}$-wave front set [44] - which was seen to be equivalent to the sc-wave front set of [102] - the homogeneous wave front set of [111], a global wave front set in terms of the Shubin calculus in [82], named Gabor wave front set in [121], and many others, like the aforementioned qsc-wave front 
set of [153] and the metaplectic wave front set [149].

For some of these notions, it is unclear to make out how they relate to the others. In this thesis, we indicate how to relate some of these different approaches with which one characterizes global singularities and that several of these are indeed equivalent and may be traced back to two fundamental notions of global wave front set, which we denote by $\mathrm{WF}_{\mathrm{SG}}$ and $\mathrm{WF}_{\mathrm{G}}$.

We establish a firm understanding, with reference to the underlying geometry, of these two notions, which capture microlocal deviation of tempered distributions from being $\mathscr{S}$-regular, meaning from being rapidly decreasing and smooth. We revisit certain constructions known for the classical wave front set, and relate these to known properties of the global wave front sets. We also establish (to the best of our knowledge) new properties of these wave front sets, such as by constructing for any possible given wave front set a distribution which admits precisely these singularities. We achieve this by suitably generalizing classical constructions.

The construction that may be considered our main result is that we establish a theory of tempered distributions given by oscillatory integrals, which arise as (micro-) local expressions of Lagrangian distributions, and characterize their singularities. In the classical theory, these classes of distributions are actually associated to their wave front set, which may be given in terms of geometric objects, that is conic Lagrangian submanifolds of $T^{*} X$. We establish in great detail how to generalize this class of manifolds to the global setting, which is particularly challenging since the structure of the compactification of $T^{*} X$ used is that of a manifold with corners. This theory has been achieved in collaboration with Coriasco in [49, 50]. We then relate these to the known classes of Lagrangian (see [79]) and Legendrian (see [70, 71, 107]) submanifolds used to obtain classes of distributions.

As another application of our study of global singularities, we establish for both $\mathrm{WF}_{\mathrm{SG}}$ and $\mathrm{WF}_{\mathrm{G}}$ microlocal existence criteria for the extension of operations on functions and of operators $\mathscr{S} \mapsto \mathscr{S}^{\prime}$ to tempered distributions, generalizing and complementing results of [81, 82]. In particular, we study "classic" operations such as the pairing and the pull-back of distributions, but also give a - to the best of our knowledge - novel treatment of the Weyl product and the action of Weyl-quantized operators with distributional symbols on distributions.

We note that the microlocal approach to geneneralize operations to distributions has been employed to great success in recent local constructions from constructive 
perturbative quantum field theory, see e.g. [4, 16, 74, 75, 112, 152]. We discuss some of these expressions, in particular the two-point function and (twisted) powers thereof, and show how these may be defined from the microlocal point of view. Since our methods take the global nature of singularities into account, they may be applicable, in the future, to problems involving growth singularities or even both frequency as well as growth singularities, such as the adiabatic limit, see e.g. [59], as well as the IR/UV-mixing problem, see [4].

We also generalize another notion that arises in these constructions, the Steinmann scaling degree, to the global setting. This notion is useful to extend distributions defined on some subspace of test functions vanishing at a submanifold to general tempered distributions and to classify the amount of possible extensions. In the aforementioned constructions, this method is used to renormalize - that is extend - products of distributions that are not canonically defined by microlocal existence criteria.

We establish this way of extending distributions, which is a generalization of Hadamard's method of finite parts, see [16, 108, 112], for tempered distributions. We complement this analysis by discussing in detail how these scaling techniques relate to some of the methods used to study global singularities, with reference to a symbol map defined for general distribution as introduced by Weinstein [148].

In the course of our analysis, we establish several techniques that may be employed to study global singularities of tempered distributions. It is a natural question if and how these methods translate to different functional settings, such as modulation spaces - and in particular (weighted) Sobolev spaces - as well as Gelfand-Shilov spaces $\mathscr{S}_{\theta}$. We address this along the lines of our analysis, and in the latter case include a discussion on which of these microlocal methods may be generalized to the quasi-analytic, or hyper-analytic context, that is for $\frac{1}{2} \leq \theta<1$.

\section{Outline and main results}

The thesis is organised as follows:

In Section 1 we establish the geometric set-up of radial compactification of $\mathbb{R}^{d}$ and $T^{*} \mathbb{R}^{d}$ in Section 1.1.2 and indicate how cut-offs "at infinity" may be constructed. In particular, we carry out Construction 1.5, which resembles a dyadic decomposition or Littlewood-Paley partition of unity and may be used to produce symbols elliptic at a point.

In Section 1.2 we introduce the symbol classes needed for our purpose of analysing 
tempered distributions. We introduce Shubin-type symbols in Section 1.2.2 and SG-symbols in Section 1.2.3 from a unified viewpoint with reference to the aforementioned compactifications, with special emphasis on the subclass of polyhomogeneous SG-symbols in Section 1.2.4. We list elements of the associated pseudodifferential calculi in an appendix, that is Section A.3. We continue this in Section A.3.3 by recalling the notion of localization operator and by indicating the interplay between pseudo-differential analysis and time-frequency analysis.

In Section 2 we analyse the notions of wave front sets associated to the calculi under study. We begin by introducing the SG-wave front set in Section 2.2. We establish that it may be understood as a generalization of Hörmander's classical wave front set, discuss examples and study systematically how constructions known in the classical setting may be converted to the global one. In particular we construct, in Proposition 2.11, a tempered distribution with arbitrary assigned wave front set. We continue this train of thoughts and establish, in Section 2.3.1, how the SG-wave front set may be characterized in terms of a generalized FBI-transform. The the main theorem of this section is the characterization of the corner component $\mathrm{WF}_{\mathrm{SG}}^{\psi e}$ in Theorem 2.22. In Section 2.4.1 we make use of this characterization and show how the SG-wave front set may be used to generalize operations from functions in a canonical way to distributions. Our main results of this section are the characterization of the product, Corollary 2.47 1 and a construction to establish general pull-backs by linear maps. We also study translation invariant distributions and remark that the SG-wave front set may be used to prove a microlocal version of the Theorem 0 of Epstein and Glaser for tempered distributions.

We establish a similar analysis for the G-wave front set in Section 2.5. After introducing the notion and listing basic properties of it, we proceed to give a time-frequency theoretical description of it in Section 2.6, in particular by use of localization operators and a parameter-dependent version of the short-time Fourier transform. As a main result we establish the equality of the G-wave front set with Nakamura's notion of homogeneous wave front set in Theorem 2.74, which may be seen as a semi-classical description of G-type singularities. Again, we construct

\footnotetext{
${ }^{1}$ We mention already at this point that this result has already been stated in some unpublished lecture notes by R.B. Melrose, [106]. Our method of proof, however, differs from the one used therein. Our treatment of tensor products is to the best of our knowledge new.
} 
a distribution with assigned wave front set in Theorem 2.68. Most of these results were obtained in collaboration with Wahlberg in [130]. We conclude by studying operations in terms of the G-wave front set, recalling and complementing the results of Hörmander [82], in Section 2.7.

Having introduced these notions of wave front sets, we proceed to study applications in Section 3. In Section 3.2 we present the results of a joint project with Coriasco, based on [49, 50]. We establish a theory of tempered oscillatory integrals and SG-Lagrangians with suitable behaviour at infinity. The results contained in this section may be considered the main results of this thesis. The analysis is carried out by first introducing the "local picture", that is the definition of tempered oscillatory integrals in Theorem 3.9 and a classification of their singularities in Theorem 3.18. We then proceed to discuss this from a geometric point of view and define SG-Lagrangians in Section 3.2.4. We establish in Theorem 3.34 that the structures arising in Theorem 3.18 for non-degenerate $\mathrm{SG}_{\mathrm{cl}}^{1,1}$-phase functions are precisely of this type and that conversely, locally, every SG-Lagrangian may be parametrized by such a phase function in Theorem 3.35.

In Section 3.3 we discuss how Weinstein's construction of a symbol for general distributions may be formulated from the point of view of our analysis and may be generalized to the SG-setting. We also generalize the notion of Steinmann's scaling degree known from quantum field theory to the global setting of tempered distributions and study extensions of tempered distributions defined "everywhere except at a subspace" in Proposition $\mathbf{3 . 5 2}$.

We continue our study of operations on tempered distributions in terms of the G-wave front set in Section 3.4 and show how the notion may be used to define twisted products, in particular the Weyl product, of distributions in Proposition 3.58. We also discuss Weyl operators with distributional symbols and extensions of such to distributions in Proposition 3.63 .

In Section 3.5, we discuss applications to the theory of quantum fields. For that, we first study, in Section 3.5.2, a distribution known as the two-point function $2^{2}$ as an oscillatory integral and obtain its SG-wave front set in Corollary 3.67. In Section 3.5.3, we mention how certain (twisted) products of distributions known

\footnotetext{
${ }^{2}$ To be precise: we study the two-point function from scalar bosonic free quantum field theory on flat Minkowski spacetime.
} 
from perturbative quantum field theory may be defined and studied from the microlocal viewpoint.

In Section 4.1, we discuss, as an outlook, how and under which assumptions the previous constructions may be translated to the functional setting of ultradistributions of type $\mathscr{S}$ and highlight some of the features that arise in these spaces, depending on the imposed type of regularity. In particular we mention an analogue of the G-wave front set suitable for the analysis of singularities of ultradistributions in the quasi-analytic case, based on a joint project with Cappiello [22]. This naturally leads over to a detailed outlook in Section 4.2, where several possible directions into which our analysis may be pursued further are discussed.

Some preliminaries are collected in Appendix A.1. Therein, we fix our basic notation and recall established facts on tempered distributions known from the literature in Section A.2. We put special emphasis on their behaviour under transformations known from time-frequency analysis in Section A.2.1.

\section{Acknowledgements}

Many people supported me while writing this thesis and I would like to express my gratitude to all of them. Though I cannot name them all here, I would still like to single out a few.

Special thanks go out to my supervisor Dorothea Bahns, who was always there for any question I might have had - academic and non-academic. I am grateful for many valuable discussions, for her trust in me and for her constant encouragement. I would also like to thank my secondary supervisor, Ingo Witt, especially for his many seminars and lectures, in which I first learned of pseudo-differential analysis and for introducing me to the research community, in particular to the group of Torino. I would also like to express my gratitude towards the remaining members of the commission, to Gert Lube, Karl-Henning Rehren, Thomas Schick and Max Wardetzky.

I would further like to thank several other people whom I met in Göttingen for the very enjoyable time and atmosphere, in particular Annika, Chiara, Fabian, Gennaro, Gerrit, Jochen, Luca, Michal, Philipp and Thang. Special thanks go out to Matthias, to whom I am very grateful for pointing out several typos during the proof-reading stage of this thesis. Towards my co-authors and guests Daniel, Marco, Massimo, Nenad, Patrik, Sandro and Viet I feel deep gratitude. It was a 
great pleasure to work with them, and I am particularly grateful for their introducing me to many concepts that later became parts of this thesis.

To all of my dear friends and colleagues whom I met in Torino, I'd like to express my deepest gratitude for their hospitality and friendliness, during my stays and in between. Apart from the ones I already mentioned I would like to single out Luigi Rodino, for invaluable advice and making my stay possible, as well as Chiara, Giovanni, Luigi Riba and Ubertino. Grazie mille!

I would also like to thank my new colleagues in Hannover, in particular Elmar Schrohe and his work group, for the warm welcome I received there.

Last but not least my thanks go out to all of my other friends, my family and above all others Katja for their patient, unconditional and simply invaluable support.

This work was supported by the German Research Foundation (Deutsche Forschungsgemeinschaft) through the Institutional Strategy of the Georg-August Universität Göttingen, in particular through the research training group GRK 1493 and the Courant Research Center "Higher Order Structures in Mathematics", as well as by the German Academic Exchange Service (Deutscher Akademischer Austauschdienst) within the framework of a "DAAD Doktorandenstipendium". It was further supported by the German National Academic Foundation "Studienstiftung des deutschen Volkes". All the support, both financial and non-financial, is gratefully appreciated.

This document was type-set in $\mathrm{LT}_{\mathrm{E}} \mathrm{X}$ using texmaker, the figures were created in TikZ and gnuplot. 


\section{Preliminaries}

\subsection{Compactification of Euclidean space.}

This thesis is devoted to the microlocal analysis of tempered distributions. As such, we assume familiarity of the reader with Schwartz functions, tempered distributions and the concepts of classical microlocal analysis. However, for the reader's convenience and to be able to clearly refer to them in the following, some basic facts on Schwartz functions and tempered distributions are collected in the Appendix, see Section A.2, where we also fix notation. We strongly advise the reader to briefly familiarize himself with the concepts included therein, since we have also included some maybe lesser-known concepts, such as those from time-frequency analysis in Section A.2.1. We have further collected a list of recurring symbols in Appendix B.

In the following, we will now introduce the tools needed to study singularities of tempered distributions. A distribution $u \in \mathscr{S}^{\prime}\left(\mathbb{R}^{d}\right)$ is globally $\mathscr{S}$-regular if its action on all test functions $f \in \mathscr{S}\left(\mathbb{R}^{d}\right)$ may be represented by the pairing with some $g \in \mathscr{S}\left(\mathbb{R}^{d}\right)$, that we have $u(f)=\langle g, f\rangle$. As such, a singularity of $u$ is any deviation from being smooth and rapidly decaying. Since rapid decay cannot be measured in some bounded neighbourhoods of a point, we have to first introduce means to describe and localize "points at infinity". This will be achieved in the next section.

\subsubsection{Radial compactification of Euclidean space.}

In order to properly encode "points at infinity", we compactify $\mathbb{R}^{d}$, i.e. embed it into a compact manifold (with boundary or even corners) and call elements of the boundary points at infinity. Here, we use the compactification of [57, Chap. 8.2], which is closely related to the stereographic, or also radial, compactification (cf. [102, 107]), see also [89]. The difference between this compactification and the directional compactification used in [36, Chap. 4]: $:^{3}$ is that they impose a different differential structure at infinity, consult also [153.

Construction 1.1 (Radial compactification of $\mathbb{R}^{d}$ ).

(1) Embedding of $\mathbb{R}^{d}$ into $\mathbb{B}^{d}$ :

We embed $\mathbb{R}^{d} \hookrightarrow \mathbb{B}^{d}$ (see Figure 1) by use of some $\iota$, a diffeomorphism

${ }^{3}$ Consult also [128]. In some unpublished lecture notes by Melrose [106] it is called quadratic radial compactification. 
$\mathbb{R}^{d} \rightarrow\left(\mathbb{B}^{d}\right)^{o}$ that is given by

$$
x \mapsto \frac{x}{|x|}\left(1-\frac{1}{|x|}\right) \text { for }|x|>3 .
$$

Consequently, the inverse $\iota^{-1}:\left(\mathbb{B}^{d}\right)^{o} \rightarrow \mathbb{R}^{d}$ is given, for $2 / 3<|y|<1$, by $y \mapsto \frac{y}{|y|}(1-|y|)^{-1}$.

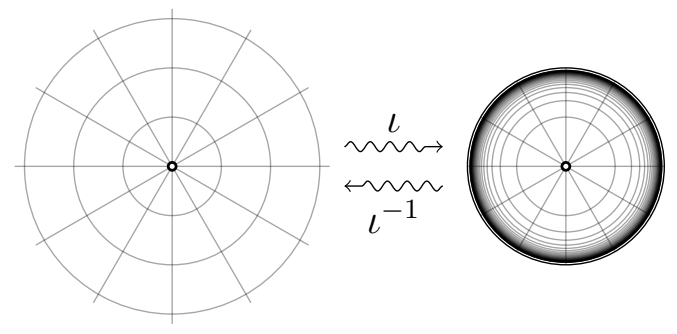

FiguRE 1. Radial compactification of $\mathbb{R}^{d}$

(2) "Points at infinity":

We then identify $\partial \mathbb{B}^{d}=\mathbb{S}^{d-1}$ as the "points at infinity". We note that each element of $x_{0} \in \mathbb{R}^{d} \backslash\{0\}$ can be assigned a corresponding boundary element "in the direction of $x_{0}$ " via $\frac{x_{0}}{\left|x_{0}\right|} \in \mathbb{S}^{d-1}$. Elements on the same ray in $\mathbb{R}^{d}$ are identified with the same boundary element, and this may be seen as a (canonical) representative of the equivalence class of $x$ in $\left(\mathbb{R}^{d} \backslash\{0\}\right) / \sim$, which we will denote in the following by the symbolic expression ${ }^{4} x_{0} \infty$, where the equivalence relation $\sim$ is given by

$$
x \sim \lambda x \text { for } \lambda>0 .
$$

When working on asymptotic problems in $\mathbb{R}^{d}$, one may thus choose to either work on $\mathbb{B}^{d}$ itself or, in light of the isomorphism $\iota$, on $\mathbb{R}^{d} \sqcup \mathbb{S}^{d-1} \cong \mathbb{R}^{d} \sqcup\left(\left(\mathbb{R}^{d} \backslash\{0\}\right) / \sim\right)$. In the following, we will often make implicit use of this identification $\mathbb{R}^{d} \cong\left(\mathbb{B}^{d}\right)^{o}$, $\left(\left(\mathbb{R}^{d} \backslash\{0\}\right) / \sim\right) \cong \partial \mathbb{B}^{d}$.

(3) Associated conifications:

Instead of considering subsets of $\left(\left(\mathbb{R}^{d} \backslash\{0\}\right) / \sim\right) \cong \mathbb{S}^{d-1}$, we often simply consider conic subsets of $\mathbb{R}^{d} \backslash\{0\}$. To identify these with subsets of $\partial \mathbb{B}^{d}$, we use the following notation. Let $U \subset \partial \mathbb{B}^{d}$. Then the cone with base $U$,

\footnotetext{
${ }^{4}$ We have essentially adapted the notation of [88] for "points at infinity".
} 
$\Gamma(U)$ is defined as

$$
\Gamma(U):=\left\{\lambda y \mid y \in U \subset \mathbb{S}^{d-1}, \lambda>0\right\} .
$$

The inverse of this map is then the projection onto the sphere in $\mathbb{R}^{d} \backslash\{0\}$.

(4) Admissible neighbourhoods:

We may view $\mathbb{B}^{d}$ as a subset of a "bigger" $\mathbb{R}^{d}$, which is not the "initial" $\mathbb{R}^{d}$ we have compactified, but we have $\mathbb{R}^{d} \cong\left(\mathbb{B}^{d}\right)^{o} \subset \mathbb{B}^{d} \subset \mathbb{R}^{d}$ a "trivial continuation across infinity". As such, $\mathbb{B}^{d}$ inherits the usual relative topology. We may use this to define fundamental systems of neighbourhoods on $\mathbb{R}^{d} \sqcup\left(\mathbb{R}^{d} \backslash\{0\}\right)$, using the identification $\iota$. A subset $U$ of $\mathbb{R}^{d} \sqcup\left(\mathbb{R}^{d} \backslash\{0\}\right)$ containing some $x_{0}$ is an admissible open neighbourhood of $x_{0}$ if either

(a) $U \cap \mathbb{R}^{d}$ is open and bounded, $U \cap\left(\left(\mathbb{R}^{d} \backslash\{0\}\right) / \sim\right)=\emptyset$

(b) $U \cap\left(\mathbb{R}^{d} \backslash\{0\}\right)$ is open, $U \cap \mathbb{R}^{d} \backslash\{0\}$ is conic and

$$
\left[\iota\left(U \cap \mathbb{R}^{d}\right)\right] \cup\left[\Gamma^{-1}\left(U \cap\left(\mathbb{R}^{d} \backslash\{0\}\right)\right)\right] \subset \mathbb{B}^{d} \text { is open. }
$$

Note that in the second case all possible boundary points included in an open set (the "exit points"), are determined as limit points of the interior points, in view of $\Gamma^{-1}\left(U \cap\left(\mathbb{R}^{d} \backslash\{0\}\right)\right) \subset \overline{\iota\left(\operatorname{pr}_{\mathbb{R}^{d}}(U)\right)}$. In light of the angle-preserving definition of $\iota$, this amounts to equipping $\mathbb{R}^{d}$ with a conic structure at infinity.

In the following, when we localize in an open neighbourhood around a given point, we always mean that we pick an appropriate admissible neighbourhood around it.

We may view neighbourhoods of a point at infinity simply as subsets of $\mathbb{R}^{d}$ : an open neighbourhood of a point at infinity given by some representative $x_{0} \in \mathbb{R}^{d} \backslash\{0\}$ is then the restriction of an open subset of type $4 \mathrm{~b}$, , containing $x_{0}$, to $\mathbb{R}^{d}$. Usually, it is enough to consider conic subsets of $\mathbb{R}^{d}$ containing the ray represented by $x_{0}$, intersected with $\left(\mathbb{B}_{R}^{d}\right)^{c}=\left\{x \in \mathbb{R}^{d}|| x \mid>R\right\}$ for some $R>0$.

In Section 1.1.2, this will allow us to localize around "infinite arguments" using functions on $\mathbb{R}^{d}$.

(5) Smooth structure:

As a subset of $\mathbb{R}^{d}$, the unit ball $\mathbb{B}^{d}$ may even be equipped with a $\mathscr{C}^{\infty}$ structure and viewed as a smooth manifold with corners, cf. [98, Chap. 1]. 
The reason why we work in the category of manifolds with corners and not simply manifolds with boundary, is that we will later also study products of balls and the category of manifolds with boundaries is not closed under direct products.

A smooth function on this manifold $\mathbb{B}^{d}$ is then a function that is smooth in the interior, with the usual notion of smoothness on open subsets of $\mathbb{R}^{d}$, and whose derivatives are bounded on all compact subset of $\left(\mathbb{B}^{d}\right)^{o}$.

(6) Boundary defining function:

We now choose in addition to $\iota$ a smooth function $h: \mathbb{B}^{d} \rightarrow[0, \infty)$ satisfying

$$
\left\{\begin{array}{ll}
h(y)=|y| & \text { for } \frac{2}{3}<|y|<1 \\
1-h(y) \neq 0 & \text { for }|y|<\frac{2}{3}
\end{array} .\right.
$$

Then $\tilde{y}=1-h(y)$ is a boundary defining function on $\mathbb{B}^{d}$, meaning a positive smooth function that vanishes at the boundary and only there.

(7) Schwartz functions:

The Schwartz function space of rapid decay $\mathscr{S}\left(\mathbb{R}^{d}\right)$ becomes, under the above identification $\iota$, the smooth functions on $\mathbb{B}^{d}$ vanishing of infinite order on the boundary of $\mathbb{B}^{d}$, denoted by $\dot{\mathscr{C}}^{\infty}\left(\mathbb{B}^{d}\right)$, see also [36, Prop. 4.1.1] and [102, Sect. 4]. This means there is an isomorphism $\iota_{\mathscr{S}}: \mathscr{S}\left(\mathbb{R}^{d}\right) \rightarrow$ $\dot{\mathscr{C}}^{\infty}\left(\mathbb{B}^{d}\right)$ given by $f \mapsto\left(\iota^{-1}\right)^{*} f$ and

$$
\dot{\mathscr{C}}^{\infty}\left(\mathbb{B}^{d}\right)=\bigcap_{j \in \mathbb{N}_{0}} \tilde{y}^{j} \mathscr{C}^{\infty}\left(\mathbb{B}^{d}\right) .
$$

If we view $\mathbb{B}^{d}$ as a subset of $\mathbb{R}^{d}$, any element of $\dot{\mathscr{C}}^{\infty}\left(\mathbb{B}^{d}\right)$ admits a trivial smooth extension (by zero) to all of $\mathbb{R}^{d}$. More generally, for functions that do not vanish on the boundary, we have Whitney's ([150], see also [81, Thm. 2.3.6]) and Seeley's extension theorem [134], which treat the extension of a function from a closed subset and in particular a half-space to all of $\mathbb{R}^{d}$. These extension theorems may be generalized to sectors, that is sets of the form $\mathbb{R}_{+}^{k} \times \mathbb{R}^{d-k}$, or even manifolds with corners such as $\mathbb{B}^{d} \times \mathbb{B}^{d}$. We quote the version of Seeley's extension theorem from the unpublished book [105, Thm. 1.4.1], see also [98, Chap. 2].

Proposition 1.2 (Seeley's extension theorem). Let $\Omega \subset \mathbb{R}^{d}$ open, $k \in \mathbb{N}_{0}$ with $k \leq d$ and let $\Omega_{0}:=\Omega \cap\left(\overline{\mathbb{R}_{+}^{k}} \times \mathbb{R}^{d-k}\right)$. Then there exists a linear continuous 
extension map $E: \mathscr{C}^{\infty}\left(\Omega_{0}\right) \rightarrow \mathscr{C}^{\infty}(\Omega)$, i.e. we have

$$
\left.E(\dot{f})\right|_{\Omega_{0}}=\dot{f} \quad \forall \dot{f} \in \mathscr{C}^{\infty}\left(\Omega_{0}\right) .
$$

(8) Tempered distributions:

Tempered distributions on $\mathbb{B}^{d}$ can therefore be identified with the extendibl $\complement^{5}$ distributions, which means elements in $\left(\dot{\mathscr{C}}^{\infty}\right)^{\prime}\left(\mathbb{B}^{d}\right)$ (cf. e.g. [83, App. B.2], [102]), meaning such $\dot{u}$ that may be obtained from a restriction $\dot{u}=\operatorname{pr}_{\mathbb{B}^{d}}^{*} u$ of a distribution $u \in \mathscr{D}^{\prime}\left(\mathbb{R}^{d}\right)$ via $\operatorname{pr}_{\mathbb{B}^{d}}: \mathbb{R}^{d} \rightarrow \mathbb{B}^{d}$ or equivalently $\langle\dot{u}, \dot{f}\rangle=\langle u, E \dot{f}\rangle$ for all $\dot{f} \in \dot{\mathscr{C}}^{\infty}\left(\mathbb{B}^{d}\right)$. Any such $u$ is called an extension of $\dot{u}$.

\subsubsection{Cut-offs and cut-offs "at infinity".}

Having introduced smooth functions on the compactification of $\mathbb{R}^{d}$, we now establish a notion of partition of unity or cut-offs respecting the structure "at infinity". These will be one of our main tools to (micro-)localize in the following. We note that notions of cut-offs at infinity and structure-preserving diffeomorphisms have been introduced in the more general context of SG-manifolds and in particular socalled manifolds with conic ends (of which $\mathbb{R}^{d}$ is the simplest example), see [128] and also [36, Chap. 4.2]. In the course of our analysis, we will use two ways of looking at these localizers, and therefore establish both in detail.

Construction 1.3 (Cut-offs (at infinity)).

Definition in terms of the isomorphism $\iota$ :

A cut-off around a point $y_{0}$ in $\mathbb{B}^{d}$, denoted $\phi^{y_{0}}$, is a positive element of $\mathscr{C}^{\infty}\left(\mathbb{B}^{d}\right)$ with $\left\|\phi^{y_{0}}\right\|_{L^{\infty}\left(\mathbb{B}^{d}\right)}=1$ that is equal to 1 in an open neighbourhood $U$ of $y_{0}$ and vanishes outside a bigger neighbourhood $V$ (with $U \subset V)$.

Let $x_{0}$ the corresponding point in $\mathbb{R}^{d} \sqcup\left(\left(\mathbb{R}^{d} \backslash\{0\}\right) / \sim\right)$, that is $x_{0}=\iota^{-1}\left(y_{0}\right)$ in case $y_{0} \in\left(\mathbb{B}^{d}\right)^{o}$ and if $y_{0} \in \partial \mathbb{B}^{d}$, then $x_{0}=y_{0} \infty \in\left(\mathbb{R}^{d} \backslash\{0\}\right) / \sim$.

- If $y_{0} \in\left(\mathbb{B}^{d}\right)^{o}$, and $V \cap \partial \mathbb{B}^{d}=\emptyset$, we see that $\iota^{*} \phi^{y_{0}}=\phi^{x_{0}} \in \mathscr{C}_{c}^{\infty}\left(\mathbb{R}^{d}\right)$ is a "standard" cut-off around $x_{0}$.

- If $y_{0} \in \partial \mathbb{B}^{d}$, then $\phi^{x_{0}}=\iota^{*} \phi^{y_{0}}$ is an asymptotic cut-off or cut-off "at infinity" around $x_{0}$.

The latter case is highlighted in Figure 2. More generally, given arbitrary open subsets $U, V \subset \mathbb{B}^{d}$ such that $\bar{U} \subset V$, we can construct a positive smooth function

${ }^{5}$ Using e.g. the Hahn-Banach Theorem. 
$\phi_{U}: \mathbb{B}^{d} \rightarrow \mathbb{R}$ such that $\left.\left(\phi_{U}\right)\right|_{U} \equiv 1$ and $\left.\left(\phi_{U}\right)\right|_{V^{c}} \equiv 0$. As a special case we obtain excision functions $\chi$, meaning positive functions satisfying $\chi \equiv 1$ in a neighbourhood of $\partial \mathbb{B}^{d}$ and $\chi \equiv 0$ in a neighbourhood of 0 .

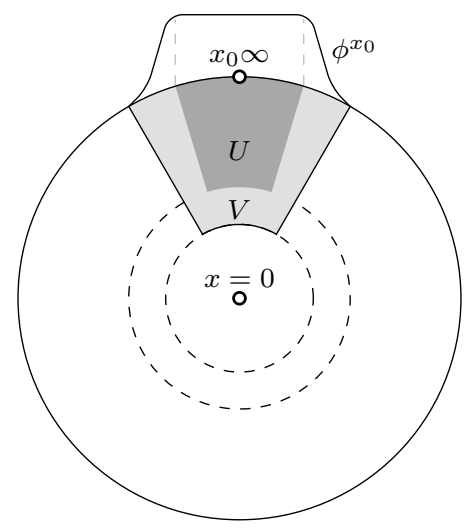

Figure 2. A model cut-off at infinity

In the previous approach, we have introduced these cut-offs with reference to the smooth structure on the compactification. Conversely (as in [106]) one may introduce these on $\mathbb{R}^{d}$ without reference to $\iota$, which we will do in the following.

Definition in terms of model cases:

Cut-offs and asymptotic cut-offs can also be constructed on $\mathbb{R}^{d}$ by considering some model cases:

- For a finite point $x_{0} \in \mathbb{R}^{d}$, we consider "standard" cut-offs, that is positive functions $\phi^{x_{0}} \in \mathscr{C}_{c}^{\infty}\left(\mathbb{R}^{d}\right)$ with $\left\|\phi^{x_{0}}\right\|_{\infty}=1$ such that $\phi^{x_{0}} \equiv 1$ in a neighbourhood of $x_{0}$ and $\phi^{x_{0}} \equiv 0$ for $\left|x-x_{0}\right|>R$ for some $R>0$.

- From cut-offs $\phi^{0}$, excision functions $\chi \in \mathscr{C}^{\infty}\left(\mathbb{R}^{d}\right)$ are constructed via $\chi=$ $1-\phi^{0}$.

- More generally, for any point at infinity given by a representative $x_{0} \infty$ in $\mathbb{S}^{d-1} \cong\left(\left(\mathbb{R}^{d} \backslash\{0\}\right) / \sim\right)$, we can consider a positive function $\psi \in \mathscr{C}^{\infty}\left(\mathbb{S}^{d-1}\right)$, which satifies $\|\psi\|_{\infty}=1$ and $\psi \equiv 1$ in a neighbourhood of the representative $x_{0} \infty$. We extend such a function homogeneously to all of $\mathbb{R}^{d} \backslash\{0\}$ and denote it by the same symbol $\psi$. Applying some excision function $\chi$, we 
obtain a smooth function $\phi^{x_{0}}: \mathbb{R}^{d} \rightarrow \mathbb{R}$ via

$$
x \mapsto \begin{cases}\chi(x) \psi\left(\frac{x}{|x|}\right) & x \neq 0 \\ 0 & x=0,\end{cases}
$$

which provides another approach of constructing a cut-off at infinity or asymptotic cut-offs.

We notice that we may guarantee during the construction that an asymptotic cutoff vanishes on any chosen bounded neighbourhood of 0 . By (locally finite) convex sums of such cut-offs we may then localize any open set as in $4 \mathrm{~b}$ ) of Construction 1.1 .

In either approach, cut-offs at infinity viewed as functions on $\mathbb{R}^{d}$ satisfy ${ }^{6} \forall \alpha \in \mathbb{N}_{0}^{d}$

$$
\left|\partial_{x}^{\alpha} \phi^{x_{0}}(x)\right| \lesssim\langle x\rangle^{-|\alpha|}
$$

which may be used to prove that they yield multipliers for the Schwartz space. $7^{7}$ Furthermore, in some admissible neighbourhood $U$ of $x_{0}$ they satisfy the ellipticity condition

$$
\left|\phi^{x_{0}}(x)\right| \gtrsim\langle x\rangle^{0} \quad \forall x \in U .
$$

Being able to localize distributions in different domains via cut-offs, it is often of importance to estimate the distance of their "arguments" from a given set. For that, one uses a standard estimate, obtained by scaling:

Lemma 1.4. Let $U, V \subset\left(\mathbb{B}^{d}\right)^{o}$ s.t. the closures of $U$ and $V$ in $\mathbb{B}^{d}$ satisfy $\bar{U} \cap \bar{V}=\emptyset$. Then for all $x_{1} \in \mathbb{R}^{d} \cap \iota^{-1}(U)$ and $x_{2} \in \mathbb{R}^{d} \cap \iota^{-1}(V)$ we have $\left|x_{1}-x_{2}\right| \gtrsim\left|x_{1}\right|+\left|x_{2}\right|$.

Sometimes it is necessary to impose precise bounds on (derivatives) of cut-offs at infinity with respect to the distance to the origin. In the following, we will establish another construction of how to relate cut-offs at infinity and such at finite arguments. We first work with $d=1$. The construction is (schematically) shown in Figure 3 and is similar to a dyadic decomposition, meaning the cutoffs used in Littlewood-Paley analysis, the prototype of wavelet analysis (see e.g. [108, 146]).

\footnotetext{
${ }^{6}$ For the first approach, this will become evident is Section 1.2 .3

${ }^{7}$ In the first construction, the multiplier property may be also concluded from the fact that $\mathscr{C}^{\infty}\left(\mathbb{B}^{d}\right) \cdot \dot{\mathscr{C}}^{\infty}\left(\mathbb{B}^{d}\right) \subset \dot{\mathscr{C}}^{\infty}\left(\mathbb{B}^{d}\right)$.
} 


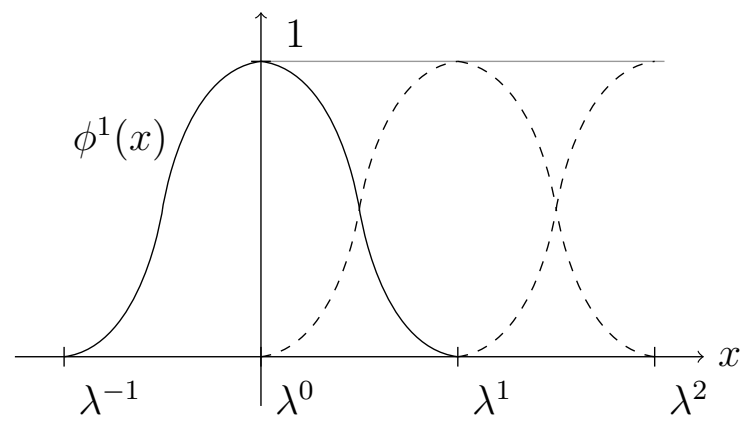

Figure 3. Construction of an asymptotic cut-off by summation and dilation

Construction 1.5. Pick a positive cut-off around $0 \in \mathbb{R}, \phi^{0}$, supported in $[-1,1]$, such that $\forall x \in[-1,0]$ we have $\phi^{0}(x)+\phi^{0}(x+1)=1$ and in particular $\phi^{0}(0)=1$ is satisfied $]^{8}$ We can then, for any $\lambda>1$ consider $\phi^{1}(x)=\phi^{0}\left(\log _{\lambda}(x)\right)$, which yields (trivially extended by 0 to all of $\mathbb{R}$ ) a smooth function, compactly supported in $\left[\lambda^{-1}, \lambda\right]$, which is positive and satisfies $\phi^{1}(1)=1$. We can now, for any $N \in \mathbb{N}$, construct the sum

$$
\phi_{R}^{+\infty}(x)=\sum_{j=N}^{+\infty} \phi^{1}\left(\lambda^{-j} x\right) .
$$

This sum is locally finite and yields a smooth function supported in $\left[\lambda^{N-1}, \infty\right)$. We can even construct, for any $M \in \mathbb{R}$, a function $a_{M}^{+\infty}$ via

$$
a_{M}^{+\infty}(x)=\sum_{j=N}^{\infty} \lambda^{j M} \phi^{1}\left(\lambda^{-j} x\right),
$$

that satisfies (using the support properties of $\phi^{1}$ )

- $a_{M}^{+\infty}$ is smooth and supported in $[R, \infty)$,

- $\left|\partial_{x}^{\alpha} a_{M}^{+\infty}(x)\right| \lesssim\langle x\rangle^{M-\alpha}$ for $\alpha \in \mathbb{N}_{0}$ and

${ }^{8}$ The existence of such a function can be demonstrated as follows:

pick a smooth positive function $f$ supported in $[-1,0]$, such as $x \mapsto \mathbb{1}_{[-1,0]} e^{-\frac{1}{1-4|x+1 / 2|^{2}}}$ (the function used in the classic demonstration of the existence of test functions, see [81, Lem. 1.2.3]). With $C:=\int_{-1}^{0} f(x) d x$ we define

$$
\phi^{0}(x)= \begin{cases}C^{-1} \int_{-1}^{x} f(y) d y & x \leq 0 \\ 1-\phi^{0}(x-1) & 0<x \leq 1 \\ 0 & 1<x\end{cases}
$$

which satisfies all claimed properties. 
- For $x>\lambda^{N}$ we have $\lambda^{-1} x^{M}<\left|a_{M}^{+\infty}\right|<\lambda x^{M}$ and in particular we obtain $\left|a_{M}^{+\infty}(x)\right| \gtrsim\langle x\rangle^{M}$ for $x>\lambda^{N-1}$.

Figure 4 shows a (schematic) example for this construction in the case $\lambda=2$, $M=1, N=1$, where the grey "bumps" represent the functions $2^{j} \phi^{1}\left(2^{-j} x\right)$. Note that for $M=0$ this construction yields an asymptotic cut-off $a_{0}^{\infty}=\phi^{\infty}$ with the prescribed bounds from below and above around $+\infty$.

By reflection, we can obtain functions with the same properties around $-\infty$.

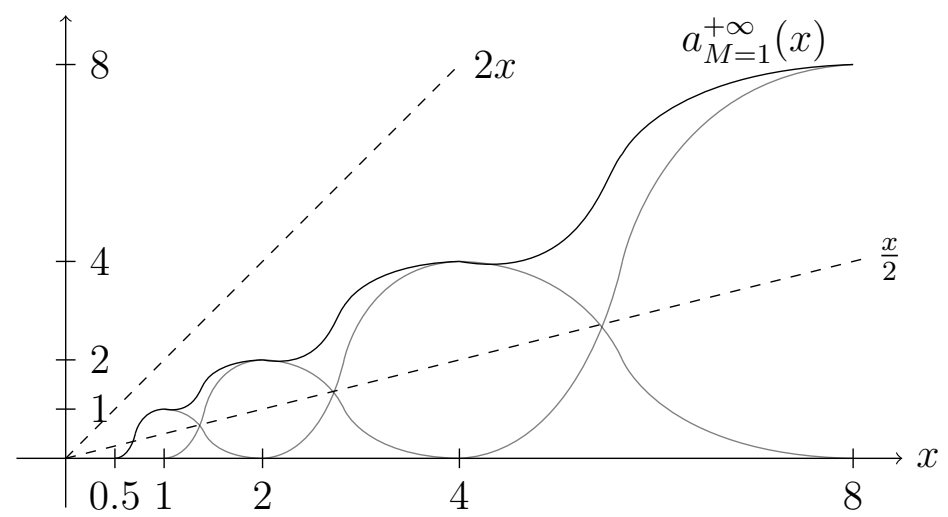

Figure 4. Construction of a function $a_{M=1}^{+\infty}$ for $\lambda=2, N=1$

More generally, we can repeat the construction in higher dimensions by using polar coordinates $x=r \cdot \eta$, where $\eta \in \mathbb{S}^{d-1}$ and $r \in[0, \infty)$, and setting

$$
a_{M}^{\eta_{0} \infty}(x)=\sum_{j=N}^{\infty} \lambda^{j M} \phi^{1}\left(\lambda^{-j} r\right) \phi^{\eta_{0}}(\eta),
$$

where $\phi^{\eta_{0}}$ is some cut-off around $\eta_{0}$ as an element of $\mathbb{R}^{d}$ (i.e. $\phi^{\eta_{0}}$ is compactly supported).

\subsubsection{Singularities at infinity.}

The notions of cut-offs at infinity and the compactification of $\mathbb{R}^{d}$ can be used to give an extension of the notion of (singular) support to tempered distributions, see also [106].

Definition 1.6 (Cone (singular) support). The cone support of $u \in \mathscr{S}^{\prime}\left(\mathbb{R}^{d}\right)$ is defined, using (asymptotic) cut-offs, in terms of its complement. We first give the 
definition of $\operatorname{Csp}(u)$ as a subset of $\mathbb{R}^{d} \sqcup \mathbb{R}^{d} \backslash\{0\}$ in terms of its complement:

$$
\operatorname{Csp}(u)^{c}=\left\{x_{0} \in \mathbb{R}^{d} \sqcup\left(\mathbb{R}^{d} \backslash\{0\}\right) \mid \exists \phi^{x_{0}} \text { s.t. } \phi^{x_{0}} u \equiv 0\right\}
$$

Correspondingly, we define the cone singular support in $\mathbb{R}^{d} \sqcup \mathbb{R}^{d} \backslash\{0\}$, Css $(u)$,

$$
\operatorname{Css}(u)^{c}=\left\{x_{0} \in \mathbb{R}^{d} \sqcup\left(\mathbb{R}^{d} \backslash\{0\}\right) \mid \exists \phi^{x_{0}} \text { s.t. } \phi^{x_{0}} u \in \mathscr{S}\left(\mathbb{R}^{d}\right)\right\}
$$

We denote the equivalent notions on $\mathbb{B}^{d}$ by $\widetilde{\operatorname{Csp}}(u)$ and $\widetilde{\operatorname{Css}}(u)$. In their definition, we make use of the fact that $\left(\iota^{-1}\right)^{*} u$ is an element of $\left(\dot{C}^{\infty}\right)^{\prime}\left(\mathbb{B}^{d}\right)$ :

$$
\begin{aligned}
& \widetilde{\operatorname{Csp}}(u)=\left\{y_{0} \in \mathbb{B}^{d} \mid \exists \phi^{y_{0}} \text { s.t. } \phi^{y_{0}}\left(\left(\iota^{-1}\right)^{*} u\right) \equiv 0\right\}^{c} \\
& \widetilde{\operatorname{Css}}(u)=\left\{y_{0} \in \mathbb{B}^{d} \mid \exists \phi^{y_{0}} \text { s.t. } \phi^{y_{0}}\left(\left(\iota^{-1}\right)^{*} u\right) \in \dot{\mathscr{C}}^{\infty}\left(\mathbb{B}^{d}\right)\right\}^{c} .
\end{aligned}
$$

We then have

$$
\begin{aligned}
\operatorname{Csp}(u) \cap \mathbb{R}^{d} & =\iota^{-1}\left(\widetilde{\operatorname{Csp}}(u) \cap\left(\mathbb{B}^{d}\right)^{o}\right) \\
\operatorname{Csp}(u) \cap\left(\mathbb{R}^{d} \backslash\{0\}\right) & =\Gamma\left(\widetilde{\operatorname{Csp}}(u) \cap \partial \mathbb{B}^{d}\right) \\
\operatorname{Css}(u) \cap \mathbb{R}^{d} & =\iota^{-1}\left(\widetilde{\operatorname{Css}}(u) \cap\left(\mathbb{B}^{d}\right)^{o}\right) \\
\operatorname{Css}(u) \cap\left(\mathbb{R}^{d} \backslash\{0\}\right) & =\Gamma\left(\widetilde{\operatorname{Css}}(u) \cap \partial \mathbb{B}^{d}\right)
\end{aligned}
$$

Remark 1.7. While $\widetilde{\operatorname{Csp}}(u)$ coincides with the closure of $\iota(\operatorname{supp}(u))$ in $\mathbb{B}^{d}$, this is not the case for $\widetilde{\operatorname{Css}}(u)$ and $\operatorname{singsupp}(u)$, since for the example $u=\mathbb{1}_{\mathbb{R}^{d}}$ we have $\operatorname{singsupp}(u)=\emptyset$, but $\widetilde{\operatorname{Css}}(u)=\partial \mathbb{B}^{d}$. Indeed, $\operatorname{Css}(u)$ measures "deviation from being smooth and rapidly decaying", meaning also "growth singularities".

It is in fact straight-forward to show, using a partition of unity argument, that $\operatorname{Css}(u)=\emptyset$ if and only if $u \in \mathscr{S}\left(\mathbb{R}^{d}\right)$.

To achieve more information on the nature of singularities in various functional settings, one studies microlocal resolutions of singularities, which is achieved by localizing in the spacial as well as in the frequency domain. This means that one obtains a resolution of singularities in the time-frequency plane. The space with the right transformation properties in which the resulting classical wave front set is a subset, is $T^{*} \mathbb{R}^{d}$. In the tempered setting, singularities arising at infinity also need to be accounted for. The choice for such a space is then a compactification of $T^{*} \mathbb{R}^{d}$.

\subsubsection{Two ways to compactify the cotangent space.}

The procedure of directional compactification outlined in Section 1.1.1 yields two 
natural choices to compactify the time-frequency plane $T^{*} \mathbb{R}^{d} \cong \mathbb{R}^{d} \times \mathbb{R}^{d}$, i.e. the model space used for microlocal analysis:

- one choice is $\mathbb{B}^{2 d}$, which is a compact manifold with a smooth boundary,

- the other natural choice is $\mathbb{B}^{d} \times \mathbb{B}^{d}$. Since $\mathbb{B}^{d}$ is a compact manifold with boundary, it is possible to carry out the direct product construction in the category of manifolds with corners, which yields a natural "symmetric" choice of constructing the differential structure of a compact manifold with corners of codimension 2, see [98, Chap. 2.3], on $\mathbb{B}^{d} \times \mathbb{B}^{d}$.

The boundaries of these spaces are then the G-wave front space given by

$$
\widetilde{\mathrm{W}}_{\mathrm{G}}:=\partial \mathbb{B}^{2 d}=\mathbb{S}^{2 d-1} \cong\left(\mathbb{R}^{2 d} \backslash\{0\}\right) / \sim=: \mathrm{W}_{\mathrm{G}}
$$

and the SG-wave front space, $\widetilde{\mathrm{W}}_{\mathrm{SG}}:=\partial\left(\mathbb{B}^{d} \times \mathbb{B}^{d}\right)$, cf. [36, Chap. 2.3.]. The latter may be decomposed into the union of three components and we introduce attach names to each of these, using the notation of [57, 131] in which

- the index $e$ is attached to objects associated with large behaviour in the spacial variables ("exit behaviour"),

- the index $\psi$ is attached to objects associated with large behaviour in the covariables,

- the index $\psi e$ is attached to objects associated with large behaviour in both sets of variables and covariables.

We may decompose $\widetilde{\mathrm{W}}_{\mathrm{SG}}$ as follows.

$$
\begin{aligned}
\widetilde{\mathrm{W}}_{\mathrm{SG}}= & \partial\left(\mathbb{B}^{d} \times \mathbb{B}^{d}\right) \\
= & \underbrace{\left.\left(\mathbb{B}^{d}\right)^{o} \times \mathbb{S}^{d-1}\right)}_{=: \widetilde{\mathrm{W}}_{\mathrm{SG}}^{\psi}} \cup \underbrace{\left(\mathbb{S}^{d-1} \times\left(\mathbb{B}^{d}\right)^{o}\right)}_{=: \widetilde{\mathrm{W}}_{\mathrm{SG}}^{e}} \cup \underbrace{\left(\mathbb{S}^{d-1} \times \mathbb{S}^{d-1}\right)}_{=: \widetilde{\mathrm{W}}_{\mathrm{SG}}^{\psi e}} \\
\cong \underbrace{\mathbb{R}^{d} \times\left(\left(\mathbb{R}^{d} \backslash\{0\}\right) / \sim\right)}_{=: \mathrm{W}_{\mathrm{SG}}^{\psi}} \sqcup \underbrace{\left(\left(\mathbb{R}^{d} \backslash\{0\}\right) / \sim\right) \times \mathbb{R}^{d}}_{=: \mathrm{W}_{\mathrm{SG}}^{e}} & =: \mathrm{W}_{\mathrm{SG}}^{\psi e}
\end{aligned}
$$

In the following, we will again identify subsets of $\left(\mathbb{R}^{d} \backslash\{0\}\right) / \sim$ with conic subsets of $\mathbb{R}^{d} \backslash\{0\}$, dropping the $\sim$ from the notation. Both choices impose different topologies "at infinity" on $T^{*} \mathbb{R}^{d}$, which are highlighted in Figure 5 .

Note that the classical space for microlocal analysis, $T^{*} \mathbb{R}^{d} \backslash\left(\mathbb{R}^{d} \times\{0\}\right)=\mathbb{R}^{d} \times$ 


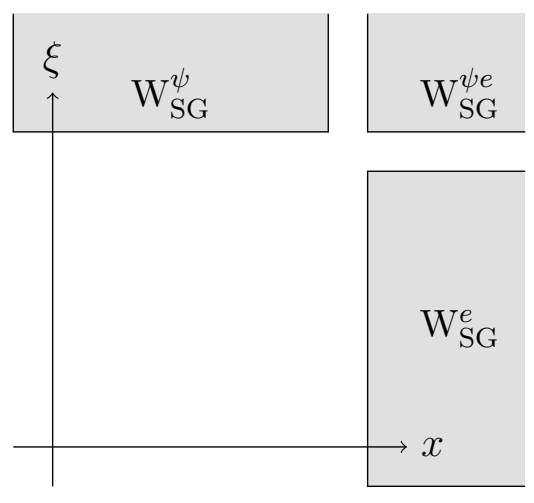

The SG-wave front space $\mathrm{W}_{\mathrm{SG}}$

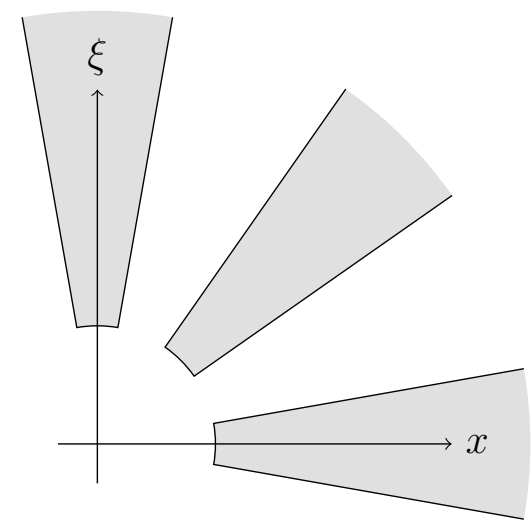

The G-wave front space $\mathrm{W}_{\mathrm{G}}$

Figure 5. Two compactifications of $T^{*} \mathbb{R}$ with "neighbourhoods at infinity" $\mathbb{R}^{d} \backslash\{0\}$ (as a set), may therefore be identified with the $\mathrm{W}_{\mathrm{SG}^{\psi}}^{\psi}$-component of the SG-wave front space.

We will now introduce symbol spaces corresponding to these compactifications. 


\subsection{Global symbol classes on Euclidean spaces.}

\subsubsection{Introduction and symbols in one set of variables.}

In this section, the basic pseudodifferential calculi that are used throughout this document are introduced. We recall, see [81, Def. 7.8.1], that the Hörmander symbols of order $m \in \mathbb{R}$ and type $(1,0)$ on $\mathbb{R}^{d}$, denoted by $\mathrm{S}_{1,0}^{m}\left(\mathbb{R}^{d} \times \mathbb{R}^{s}\right)$ for $s \in \mathbb{N}_{0}$, are characterized by those smooth functions on $\mathbb{R}^{d} \times \mathbb{R}^{s}$ such that for every compact set $K \Subset \mathbb{R}^{d}$ and all multi-indices $\alpha \in \mathbb{N}_{0}^{d}, \beta \in \mathbb{N}_{0}^{s}$ we have

$$
\left|\partial_{x}^{\alpha} \partial_{\theta}^{\beta} a(x, \theta)\right| \lesssim\langle\theta\rangle^{m-|\alpha|} \quad x \in K, \theta \in \mathbb{R}^{s} .
$$

Notation. We call the first set of variables $x \in \mathbb{R}^{d}$ the (spacial) variable and the second set $\theta \in \mathbb{R}^{s}$ the co-variable. If $s=d$, we usually denote the co-variable by the letter $\xi$ instead of by $\theta$.

In order to study tempered distributions using pseudo-differential methods, in particular to obtain pseudo-differential operators mapping $\mathscr{S} \rightarrow \mathscr{S}$, it is necessary to impose growth bounds on the symbols with respect to the (spacial) variable..$^{9}$ For that purpose, various global symbol classes have been introduced, foremost the very general Hörmander-Weyl calculus (see [80], [83, Sect. 18.5.], [94, Chap. 2]). Here, we mainly consider two types of symbols:

- SG-symbols, ${ }^{10}$ see also [36, 57, 114, 128, 131],

- as well as Shubin G-symbols ${ }^{11}$ as in [135, Chap. IV] and the references therein.

For the the definition of these SG- and G-classes of symbols in full detail, embedded in a very general calculus of global symbols on $\mathbb{R}^{d}$ suitable for the analysis of tempered distributions, the reader is referred to [113], see also [9] ${ }^{12}$ Since the attention here is limited to just the two symbol classes, they are introduced independently. We introduce both classes with reference to a symbol class "in only

\footnotetext{
${ }^{9}$ This necessity can be easily seen from the symbol $a(x, \xi)=e^{x}$.

${ }^{10}$ The SG-symbols form the class associated to the metric $h_{\mathrm{SG}}=\frac{|d x|^{2}}{\langle x\rangle^{2}}+\frac{|d \xi|^{2}}{\langle\xi\rangle^{2}}$ in the HörmanderWeyl calculus. In the approach by Melrose [102 they form (in their polyhomogeneous version) the scattering calculus.

${ }^{11}$ The G-symbols form the class associated to the metric $h_{\mathrm{G}}=\frac{|d x|^{2}+|d \xi|^{2}}{\langle(x, \xi)\rangle^{2}}$ in the Hörmander-Weyl calculus and form the isotropic calculus in the nomenclature of Melrose.

${ }^{12}$ To avoid confusion: in [113, the SG-calculus is called G-calculus, and the Shubin-calculus is denoted by $\Gamma$-calculus.
} 
one set of (co-)variables". For such a class $S^{m}\left(\mathbb{R}^{s}\right) \cong S^{m}\left(\mathbb{R}^{0} \times \mathbb{R}^{s}\right)$, the definition of Hörmander symbols class suggest the following definition, see [151].

Definition 1.8. Denote for $m \in \mathbb{R}$ by $\mathrm{S}^{m}\left(\mathbb{R}^{s}\right)$ the space of smooth functions $a \in \mathscr{C}^{\infty}\left(\mathbb{R}^{s}\right)$ that satisfy $\forall \beta \in \mathbb{N}_{0}^{s}$, called the symbols, or amplitudes, of order $m$

$$
\left|\partial_{\theta}^{\beta} a(\theta)\right| \lesssim\langle\theta\rangle^{m-|\beta|}
$$

A family of semi-norms on this space is given by

$$
\rho_{N}^{m}(a)=\sup _{\theta \in \mathbb{R}^{s}} \sum_{|\beta| \leq N}\langle\theta\rangle^{-m+|\beta|}\left|\partial_{\theta}^{\beta} a(\theta)\right| .
$$

and the induced topology turns $\mathrm{S}^{m}\left(\mathbb{R}^{s}\right)$ into a Fréchet space.

For $m^{\prime}<m$ we have that $\mathrm{S}^{m^{\prime}}\left(\mathbb{R}^{s}\right) \subset \mathrm{S}^{m}\left(\mathbb{R}^{s}\right)$ and symbol of order $m$ is called of genuine order $m$ if it is not an element of any such $\mathrm{S}^{m^{\prime}}\left(\mathbb{R}^{s}\right)$. Using this, one defines $\mathrm{S}\left(\mathbb{R}^{s}\right)=\bigcup_{m \in \mathbb{R}} \mathrm{S}^{m}\left(\mathbb{R}^{s}\right)$ as the space of all symbols and notes $\mathrm{S}^{-\infty}\left(\mathbb{R}^{s}\right):=$ $\bigcap_{m \in \mathbb{R}} \mathrm{S}^{m}\left(\mathbb{R}^{s}\right)=\mathscr{S}\left(\mathbb{R}^{s}\right)$ with the inclusion $\mathscr{S}\left(\mathbb{R}^{s}\right) \hookrightarrow \mathrm{S}^{m}\left(\mathbb{R}^{s}\right)$ being continuous.

Given a decreasing sequence $\left\{m_{j}\right\}_{j \in \mathbb{N}_{0}}$ such that $m_{j} \rightarrow-\infty$ as $j \rightarrow-\infty$ and a corresponding sequence of $a_{j} \in \mathrm{S}^{m_{j}}\left(\mathbb{R}^{s}\right)$ we write that

$$
a \sim \sum_{j=0}^{\infty} a_{j}
$$

if for all $n \in \mathbb{R}$ there exists $N \in \mathbb{N}_{0}$ such that $a-\sum_{j=0}^{N} a_{j} \in \mathrm{S}^{n}\left(\mathbb{R}^{s}\right)$. In fact we can construct (see [151, Prop. 2.1.]) for any such a given sequence $\left\{a_{j}\right\}_{j \in \mathbb{N}_{0}}$ a symbol $a \in \mathrm{S}^{m_{0}}\left(\mathbb{R}^{s}\right)$, uniquely determined modulo $\mathscr{S}\left(\mathbb{R}^{s}\right)$, such that $a \sim \sum_{j=0}^{\infty} a_{j}$.

The subspace $\mathrm{S}_{\mathrm{cl}}^{m}\left(\mathbb{R}^{s}\right)$ is formed by all classical, or polyhomogeneous, symbols, meaning those symbols that admit asymptotic expansions in terms of homogeneous functions. For its definition, denote by $\mathscr{P}^{m}\left(\mathbb{R}^{s}\right)$ the space of homogeneous functions $a \in \mathscr{C}^{\infty}\left(\mathbb{R}^{s} \backslash\{0\}\right)$ of degree $m$, that is $a(\lambda \theta)=\lambda^{m} a(\theta)$ for all $\lambda>0$.

Definition 1.9. A symbol $a \in \mathrm{S}^{m}\left(\mathbb{R}^{s}\right)$ is called classical or polyhomogeneous, if it admits a polyhomogeneous expansion, that is if there exists a sequence $\left\{m_{j}\right\}_{j \in \mathbb{N}_{0}}$ such that $m_{j} \rightarrow-\infty$ as $j \rightarrow \infty$ and a corresponding sequence of $a_{j} \in \mathscr{P}^{m_{j}}\left(\mathbb{R}^{s}\right)$ such that for any excision function $\chi^{0} \in \mathscr{C}^{\infty}\left(\mathbb{R}^{s}\right)$ we have

$$
a \sim \sum_{j=0}^{\infty}\left(\chi^{0} a_{j}\right) .
$$


The space of all classical symbols of a fixed order $m \in \mathbb{R}$ is denoted by $\mathrm{S}_{\mathrm{cl}}^{m}\left(\mathbb{R}^{s}\right)$.

It turns out that this space is a nuclear Fréchet space, cf. [151, Prop. 2.3.].

Example 1.10. Consider the cut-offs of Section 1.1.2.

- For $\theta_{0} \in \mathbb{R}^{s}$ we have $\phi^{\theta_{0}} \in \mathscr{C}_{c}^{\infty}\left(\mathbb{R}^{s}\right) \subset \mathrm{S}^{-\infty}\left(\mathbb{R}^{s}\right)$.

- A cut-off around a point at infinity $\theta_{0} \infty$ is an element of $S_{\mathrm{cl}}^{0}\left(\mathbb{R}^{s}\right)$, by 1.1 .

Example 1.11. The symbol $\theta \mapsto\langle\theta\rangle$ is polyhomogeneous, since we have

$$
\langle\theta\rangle=|\theta| \sqrt{1+\theta^{-2}}=|\theta| \sum_{j=0}^{\infty} \frac{(-1)^{j}(2 j) !}{(1-2 j)(j !)^{2}\left(4^{j}\right)} \theta^{-2 j},
$$

where the Taylor series converges for $|\theta|>1$.

The symbol space $\mathrm{S}\left(\mathbb{R}^{d}\right)$ is the natural choice of symbols on $\mathbb{R}^{0} \times \mathbb{R}^{s}$. In the following we will now introduce global symbol spaces on $\mathbb{R}^{d} \times \mathbb{R}^{s}$ for $d>0$ that correspond to the compactifications in Section 1.1.4. We have collected elements of the associated pseudodifferential calculus in Appendix A.3. The reader is strongly advised to familiarize himself with these constructions, since we have also included some maybe lesser-known facts on "local parametrices" and interplay between pseudodifferential calculi and time-frequency analysis, in particular the Weyl-Wick connection.

\subsubsection{The space of Shubin G-symbols.}

Definition 1.12 (G-symbols). A function $a \in \mathscr{C}^{\infty}\left(\mathbb{R}^{d} \times \mathbb{R}^{d}\right)$ is called G-symbol, or Shubin symbol, of order $m \in \mathbb{R}$ if it fulfils $\forall \alpha, \beta \in \mathbb{N}_{0}^{d}$ estimates of the form

$$
\left|\partial_{x}^{\alpha} \partial_{\xi}^{\beta} a(x, \xi)\right| \lesssim\langle(x, \xi)\rangle^{m-|\alpha|-|\beta|}
$$

As such, writing $\mathbb{R}^{d} \times \mathbb{R}^{d} \ni(x, \xi)=z \in \mathbb{R}^{2 d}$, we have $\mathrm{G}^{m}\left(\mathbb{R}^{d} \times \mathbb{R}^{d}\right)=\mathrm{S}^{m}\left(\mathbb{R}^{2 d}\right)$. Consequently we obtain a family of semi-norms $\left\{\rho_{N}^{m}\right\}_{N \in \mathbb{N}_{0}}$ on $G^{m}\left(\mathbb{R}^{d} \times \mathbb{R}^{d}\right)$ which turns this space into is a Frechét space.

Again it holds for any $m^{\prime}<m$ that $\mathrm{G}^{m^{\prime}}\left(\mathbb{R}^{d} \times \mathbb{R}^{d}\right) \subset \mathrm{G}^{m}\left(\mathbb{R}^{d} \times \mathbb{R}^{d}\right)$ and

$$
\mathrm{G}\left(\mathbb{R}^{d} \times \mathbb{R}^{d}\right)=\bigcup_{m \in \mathbb{R}} G^{m}\left(\mathbb{R}^{d} \times \mathbb{R}^{d}\right)
$$

denotes the space of all G-symbols. We note that exactly as for $\mathrm{S}^{m}$ we have $\bigcap_{m \in \mathbb{R}} G^{m}\left(\mathbb{R}^{d} \times \mathbb{R}^{d}\right)=\mathscr{S}\left(\mathbb{R}^{2 d}\right)$ and we are able to introduce the subclass of classical 
symbols $\mathrm{G}_{\mathrm{cl}}^{m}\left(\mathbb{R}^{d} \times \mathbb{R}^{d}\right)$ in complete analogy.

A symbol $a$ of genuine order $m$ is called G-elliptic at a point $\left(x_{0}, \xi_{0}\right) \in \mathrm{W}_{\mathrm{G}}$ if it fulfils, in an open neighbourhood $U$ of $\left(x_{0}, \xi_{0}\right)$ (in the sense of Construction 1.1 (4b)

$$
|a(x, \xi)| \gtrsim\langle(x, \xi)\rangle^{m-|\alpha|-|\beta|} .
$$

The (open) set of all points in $\mathrm{W}_{\mathrm{G}}$ such that $a$ is G-elliptic at that point is denoted by ell $l_{\mathrm{G}}(a)$. If $a$ is G-elliptic at every point, $\operatorname{ell}_{\mathrm{G}}(a)=\mathrm{W}_{\mathrm{G}}$, it is called $G$-elliptic. The (closed) complement of $\operatorname{ell}_{\mathrm{G}}(a)$ in $\mathrm{W}_{\mathrm{G}}$ is denoted by $\operatorname{char}_{\mathrm{G}}(a)$, the $\mathrm{G}$-characteristic set.

Furthermore we have:

- The pointwise product induces a continuous bilinear product

$$
\mathrm{G}^{m}\left(\mathbb{R}^{d} \times \mathbb{R}^{d}\right) \times \mathrm{G}^{m^{\prime}}\left(\mathbb{R}^{d} \times \mathbb{R}^{d}\right) \rightarrow \mathrm{G}^{m+m^{\prime}}\left(\mathbb{R}^{d} \times \mathbb{R}^{d}\right) .
$$

and the map $a(x, \xi) \mapsto\langle(x, \xi)\rangle^{m^{\prime}} a(x, \xi)$ yields an isomorphism

$$
\mathrm{G}^{m}\left(\mathbb{R}^{d} \times \mathbb{R}^{d}\right) \rightarrow \mathrm{G}^{m+m^{\prime}}\left(\mathbb{R}^{d} \times \mathbb{R}^{d}\right) .
$$

- Differentiation yields continuous maps

$$
\partial_{x}^{\alpha} \partial_{\xi}^{\beta}: \mathrm{G}^{m}\left(\mathbb{R}^{d} \times \mathbb{R}^{d}\right) \rightarrow \mathrm{G}^{m-|\alpha|-|\beta|}\left(\mathbb{R}^{d} \times \mathbb{R}^{d}\right) .
$$

\subsubsection{The space of $S G$-symbols.}

In the following the space of SG-symbols is introduced. It has the property that ellipticity of SG-symbols may be formulated with respect to the SG-wave front space.

Notation. In order to introduce the SG-calculus in a clear and efficient manner it is of importance to distinguish if at a "point at infinity" the spacial variable or the covariable is large - or both, i.e. in which boundary face of $\mathrm{W}_{\mathrm{SG}}$ the point is located. We insert a placeholder $\bullet$ if a statement is valid if we replace all all such placeholders by the same element $\bullet \in\{e, \psi, \psi e\}$.

Definition 1.13 (SG-symbols). A function $a \in \mathscr{C}^{\infty}\left(\mathbb{R}^{d} \times \mathbb{R}^{d}\right)$ is called SG-symbol or SG-amplitude of order $\left(m_{e}, m_{\psi}\right) \in \mathbb{R} \times \mathbb{R}$ if it fulfils estimates of the form

$$
\left|\partial_{x}^{\alpha} \partial_{\xi}^{\beta} a(x, \xi)\right| \lesssim\langle x\rangle^{m_{e}-|\alpha|}\langle\xi\rangle^{m_{\psi}-|\beta|} .
$$


A family of semi-norms $\left\{\rho_{N}^{m_{e}, m_{\psi}}\right\}_{N \in \mathbb{N}_{0}}$ on the space of all SG-symbols of fixed order $\left(m_{e}, m_{\psi}\right)$, denoted by $\mathrm{SG}^{m_{e}, m_{\psi}}\left(\mathbb{R}^{d} \times \mathbb{R}^{d}\right)$, is given by

$$
\rho_{N}^{m_{e}, m_{\psi}}(a)=\sup _{(x, \xi) \in \mathbb{R}^{d} \times \mathbb{R}^{d}} \sum_{|\alpha|+|\beta| \leq N}\left|\partial_{x}^{\alpha} \partial_{\xi}^{\beta} a(x, \xi)\right|\langle x\rangle^{-m_{e}+|\alpha|}\langle\xi\rangle^{-m_{\psi}+|\beta|}
$$

and $\mathrm{SG}^{m_{e}, m_{\psi}}\left(\mathbb{R}^{d} \times \mathbb{R}^{d}\right)$ equipped with these is a Frechét space.

In the following, we list some properties of these symbol spaces:

- We have the continuous inclusion $\mathrm{SG}^{m_{e}^{\prime}, m_{\psi}^{\prime}}\left(\mathbb{R}^{d} \times \mathbb{R}^{d}\right) \subset \mathrm{SG}^{m_{e}, m_{\psi}}\left(\mathbb{R}^{d} \times \mathbb{R}^{d}\right)$ for $m_{e}^{\prime} \leq m_{e}$ and $m_{\psi}^{\prime} \leq m_{\psi}$ and a symbol of order $\left(m_{e}, m_{\psi}\right)$ is called of genuine order $\left(m_{e}, m_{\psi}\right)$ if it is not in any such $\mathrm{SG}^{m_{e}^{\prime}, m_{\psi}^{\prime}}\left(\mathbb{R}^{d} \times \mathbb{R}^{d}\right)$ for $\left(m_{e}^{\prime}, m_{\psi}^{\prime}\right) \neq\left(m_{e}, m_{\psi}\right)$.

- The pointwise product induces a continuous bilinear product

$$
\mathrm{SG}^{m_{e}, m_{\psi}}\left(\mathbb{R}^{d} \times \mathbb{R}^{d}\right) \times \mathrm{SG}^{m_{e}^{\prime}, m_{\psi}^{\prime}}\left(\mathbb{R}^{d} \times \mathbb{R}^{d}\right) \rightarrow \mathrm{SG}^{m_{e}+m_{e}^{\prime}, m_{\psi}+m_{\psi}^{\prime}}\left(\mathbb{R}^{d} \times \mathbb{R}^{d}\right) .
$$

The map

$$
a(x, \xi) \mapsto\langle x\rangle^{m_{e}^{\prime}}\langle\xi\rangle^{m_{\psi}^{\prime}} a(x, \xi)
$$

yields an isomorphism

$$
\mathrm{SG}^{m_{e}, m_{\psi}}\left(\mathbb{R}^{d} \times \mathbb{R}^{d}\right) \rightarrow \mathrm{SG}^{m_{e}+m_{e}^{\prime}, m_{\psi}+m_{\psi}^{\prime}}\left(\mathbb{R}^{d} \times \mathbb{R}^{d}\right) .
$$

- Differentiation yields continuous maps

$$
\partial_{x}^{\alpha} \partial_{\xi}^{\beta}: \mathrm{SG}^{m_{e}, m_{\psi}}\left(\mathbb{R}^{d} \times \mathbb{R}^{d}\right) \rightarrow \mathrm{SG}^{\left(m_{e}-|\alpha|, m_{\psi}-|\beta|\right)}\left(\mathbb{R}^{d} \times \mathbb{R}^{d}\right) .
$$

- We denote by $\mathrm{SG}\left(\mathbb{R}^{d}\right)$ the algebra of all symbols, i.e.

$$
\mathrm{SG}\left(\mathbb{R}^{d} \times \mathbb{R}^{d}\right):=\mathrm{SG}^{\infty, \infty}\left(\mathbb{R}^{d} \times \mathbb{R}^{d}\right):=\bigcup_{\left(m_{e}, m_{\psi}\right) \in \mathbb{R}^{2}} \mathrm{SG}^{m_{e}, m_{\psi}}\left(\mathbb{R}^{d} \times \mathbb{R}^{d}\right) .
$$

We note that

$$
\mathrm{SG}^{-\infty,-\infty}\left(\mathbb{R}^{d} \times \mathbb{R}^{d}\right):=\bigcap_{\left(m_{e}, m_{\psi}\right) \in \mathbb{R}^{2}} \mathrm{SG}^{m_{e}, m_{\psi}}\left(\mathbb{R}^{d} \times \mathbb{R}^{d}\right)=\mathscr{S}\left(\mathbb{R}^{2 d}\right)
$$

Definition 1.14 (Elliptic SG-symbols). A symbol $a$ of genuine order $\left(m_{e}, m_{\psi}\right)$ is called $S G$-elliptic at a point $\left(x_{0}, \xi_{0}\right) \in \mathrm{W}_{\mathrm{SG}}$ if it also fulfils, in an open neighbourhood $U \times V \subset \mathbb{R}^{d} \times \mathbb{R}^{d}$ of $\left(x_{0}, \xi_{0}\right)$ in $\mathrm{W}_{\mathrm{SG}}$ (in the sense of Construction 1.1 (4b)),

$$
|a(x, \xi)| \gtrsim\langle x\rangle^{m_{e}-|\alpha|}\langle\xi\rangle^{m_{\psi}-|\beta|} \quad \forall(x, \xi) \in U \times V .
$$


The set of all points in $\mathrm{W}_{\mathrm{SG}}$ such that $a$ is SG-elliptic at that point is denoted by ell $_{\mathrm{SG}}(a)$. Equivalently, we may also define the notion of ellipticity at a corresponding point of $\widetilde{\mathrm{W}}_{\mathrm{SG}}$ and the set $\widetilde{\text { ell }} \mathrm{SG}_{\mathrm{G}}(a)$ as the subset of points of ellipticity $\widetilde{\mathrm{W}}_{\mathrm{SG}}$. If $a$ is SG-elliptic at every point, it is called SG-elliptic. The complement of ell $\mathrm{SG}_{\mathrm{S}}(a)$ in $\mathrm{W}_{\mathrm{SG}}$ is denoted by $\operatorname{char}_{\mathrm{SG}}(a)$, the $\mathrm{SG}$-characteristic set.

Example 1.15. Let $\left(x_{0}, \xi_{0}\right) \in \mathrm{W}_{\mathrm{SG}}$. Then, using the notation of Section 1.1.2, $a=\phi^{x_{0}} \otimes \phi^{\xi_{0}}$ is a $(0,0)$-order symbol elliptic at $\left(x_{0}, \xi_{0}\right)$ for any (asymptotic) cut-offs at $x_{0}$ and $\xi_{0}$ respectively. In particular this is true for the cut-offs of Construction 1.5 .

If $\left(x_{0}, \xi_{0}\right) \in \mathrm{W}_{\mathrm{SG}}^{\psi e}$, then such an $a$ is of genuine order $(0,0)$. Otherwise, due to its support properties, in case $\left(x_{0}, \xi_{0}\right) \in \mathrm{W}_{\mathrm{SG}}^{\psi}$ it is of order $(-\infty, 0)$, or if $\left(x_{0}, \xi_{0}\right) \in \mathrm{W}_{\mathrm{SG}}^{e}$, of order $(0,-\infty)$.

\subsubsection{Classical SG-symbols.}

A SG-symbol is called polyhomogeneous, or classical, if it admits a polyhomogeneous expansion. The shortest way to characterize this is by [151, Prop. 2.7.], where it is observed that $\mathrm{SG}_{\mathrm{cl}}\left(\mathbb{R}^{d} \times \mathbb{R}^{d}\right) \cong \mathrm{S}_{\mathrm{cl}}^{m_{e}}\left(\mathbb{R}^{d}\right) \otimes \mathrm{S}_{\mathrm{cl}}^{m_{\psi}}\left(\mathbb{R}^{d}\right)$ with the unique Fréchet tensor product, granted by nuclearity of $\mathrm{S}^{m_{e}}$.

Remark 1.16. The fact that $\mathrm{G}_{\mathrm{cl}}^{m}\left(\mathbb{R}^{d} \times \mathbb{R}^{d}\right) \cong \mathrm{S}^{m}\left(\mathbb{R}^{2 d}\right)$ corresponds to $\widetilde{\mathrm{W}}_{\mathrm{G}}=\partial \mathbb{B}^{2 d}$ and $\mathrm{SG}_{\mathrm{cl}}\left(\mathbb{R}^{d} \times \mathbb{R}^{d}\right) \cong \mathrm{S}_{\mathrm{cl}}^{m_{e}}\left(\mathbb{R}^{d}\right) \otimes \mathrm{S}_{\mathrm{cl}}^{m_{\psi}}\left(\mathbb{R}^{d}\right)$ corresponds to $\widetilde{\mathrm{W}}_{\mathrm{SG}}=\partial\left(\mathbb{B}^{d} \times \mathbb{B}^{d}\right)$.

In order to make such a polyhomogeneous expansion more explicit, we first need to introduce a number of spaces of homogeneous functions, for each component individually. In its polyhomogeneous version, the SG-calculus was mainly developed by Schulze, see [131, Chap. 1.4]. We follow the similar outline of [113, Chap. 3.1.], with slight changes in notation.

Definition 1.17 (Polyhomogeneous symbols).

- Let $\mathscr{P}_{e}^{m_{e}, m_{\psi}}$ denote the space of $a \in \mathscr{C}^{\infty}\left(\mathbb{R}^{d} \backslash\{0\} \times \mathbb{R}^{d}\right)$ such that $a$ is homogeneous of degree $m_{e}$ in the first set of variables, i.e. $\forall \lambda>0$ we have $a(\lambda x, \xi)=\lambda^{m_{e}} a(x, \xi)$ and such that for any excision function $\chi^{e} \in \mathscr{C}^{\infty}\left(\mathbb{R}^{d}\right)$ we have

$$
\left((x, \xi) \mapsto \chi^{e}(x) a(x, \xi)\right) \in \mathrm{SG}^{m_{e}, m_{\psi}}\left(\mathbb{R}^{d} \times \mathbb{R}^{d}\right)
$$


- Similarly, let $\mathscr{P}_{\psi}^{m_{e}, m_{\psi}}$ denote the space of $a \in \mathscr{C}^{\infty}\left(\mathbb{R}^{d} \times \mathbb{R}^{d} \backslash\{0\}\right)$ such that $a$ is homogeneous of degree $m_{\psi}$ in the second set of variables and such that for any excision function $\chi^{\psi} \in \mathscr{C}^{\infty}\left(\mathbb{R}^{d}\right)$ we have

$$
\left((x, \xi) \mapsto \chi^{\psi}(\xi) a(x, \xi)\right) \in \mathrm{SG}^{m_{e}, m_{\psi}}\left(\mathbb{R}^{d} \times \mathbb{R}^{d}\right) .
$$

- Finally, let $\mathscr{P}_{\psi e}^{m_{e}, m_{\psi}}$ denote the space of $a \in \mathscr{C}^{\infty}\left(\mathbb{R}^{d} \backslash\{0\} \times \mathbb{R}^{d} \backslash\{0\}\right)$ such that $a$ is separately homogeneous of degree $m_{e}$ in the first and $m_{\psi}$ in the second set of variables respectively ${ }^{13}$ and such that for any pair of excision functions $\chi^{e}, \chi^{\psi} \in \mathscr{C}^{\infty}\left(\mathbb{R}^{d}\right)$ we have

$$
\left((x, \xi) \mapsto \chi^{e}(x) \chi^{\psi}(\xi) a(x, \xi)\right) \in \mathrm{SG}^{m_{e}, m_{\psi}}\left(\mathbb{R}^{d} \times \mathbb{R}^{d}\right) .
$$

Let $a \in \mathscr{P}_{e}^{m_{e}, m_{\psi}}$. We say $a$ is $\psi$-polyhomogeneous if $a$ admits a polyhomogeneous expansion with respect to the $\xi$-variable. That means there exists some excision function $\chi^{\psi} \in \mathscr{C}^{\infty}\left(\mathbb{R}^{d}\right)$ and a sequence $\left\{a_{j}\right\}_{j \in \mathbb{N}_{0}}$ with $a_{j} \in \mathscr{P}_{\psi e}^{m_{e}, m_{\psi}-j}$ such that $a \sim \sum_{j} a_{j}$, meaning for all $N \in \mathbb{N}_{0}$

$$
\left(a-\sum_{j=0}^{N} \chi^{\psi}(\xi) a_{j}\right) \in \mathscr{P}_{e}^{m_{e}, m_{\psi}-N-1} .
$$

We call $a_{j}=\sigma_{\psi}^{m_{\psi}-j}(a)$ the $\psi$-Symbol of order $m_{\psi}-j$. We define $e$-polyhomogeneity and e-Symbol of order $m_{e}-j$, i.e. $\sigma_{e}^{m_{e}-j}(b)$ similarly, for an element of $b$ of $\mathscr{P}_{\psi}^{m_{e}, m_{\psi}}$ by interchanging the roles of the variables.

Finally, let $a \in \mathrm{SG}^{m_{e}, m_{\psi}}\left(\mathbb{R}^{d} \times \mathbb{R}^{d}\right)$ a general SG-symbol. We say $a$ is $(\psi e-)$ polyhomogeneous, or classical, if there exists some excision functions $\chi^{e}, \chi^{\psi} \in \mathscr{C}^{\infty}\left(\mathbb{R}^{d}\right)$ and two sequences $\left\{a_{j}\right\}_{j \in \mathbb{N}_{0}},\left\{b_{j}\right\}_{j \in \mathbb{N}_{0}}$ with $a_{j} \in \mathscr{P}_{\psi}^{m_{e}, m_{\psi}-j} e$-polyhomogeneous, $b_{j} \in \mathscr{P}_{e}^{m_{e}-j, m_{\psi}} \psi$-polyhomogeneous, such that $a \sim \sum_{j} a_{j}$ and $a \sim \sum_{j} b_{j}$, meaning for all $N \in \mathbb{N}_{0}$

$$
\begin{aligned}
& \left(a-\sum_{j=0}^{N} \chi^{\psi}(\xi) a_{j}\right) \in \mathrm{SG}^{m_{e}, m_{\psi}-N-1}\left(\mathbb{R}^{d} \times \mathbb{R}^{d}\right) \\
& \left(a-\sum_{j=0}^{N} \chi^{e}(x) b_{j}\right) \in \mathrm{SG}^{m_{e}-N-1, m_{\psi}}\left(\mathbb{R}^{d} \times \mathbb{R}^{d}\right)
\end{aligned}
$$

For $\psi e$-polyhomogeneous symbols, we may now define the corresponding symbols as well, setting $\sigma_{\psi}^{m_{\psi}-j}(a)=a_{j}$ and $\sigma_{e}^{m_{e}-j}(a)=b_{j}$. As the $a_{j}$ and $b_{j}$ are required to $\overline{{ }^{13} \text { This means }} \forall \lambda, \mu>0 a(\lambda x, \mu \xi)=\lambda^{m_{e}} \mu^{m_{\psi}} a(x, \xi)$. 
be polyhomogeneous, they admit expansions with to the remaining variable and we have the correspondence condition $\forall j, k \in \mathbb{N}_{0}$ :

$$
\sigma_{\psi}^{m_{\psi}-k} a_{j}=\sigma_{e}^{m_{e}-j} b_{k}=: \sigma_{\psi e}^{m_{e}-j, m_{\psi}-k}(a) \in \mathscr{P}_{\psi e}^{m_{e}-j, m_{\psi}-k} .
$$

The space of classical symbols is denoted by $\mathrm{SG}_{\mathrm{cl}}^{m_{e}, m_{\psi}}\left(\mathbb{R}^{d} \times \mathbb{R}^{d}\right)$.

By the previous discussion, we obtain a triple called the SG-principal symbol of $a$

$$
\left(\sigma_{e}^{m_{e}}(a), \sigma_{\psi}^{m_{\psi}}(a), \sigma_{\psi e}^{m_{e}, m_{\psi}}(a)\right) \in \mathscr{P}_{e}^{m_{e}, m_{\psi}} \times \mathscr{P}_{\psi}^{m_{e}, m_{\psi}} \times \mathscr{P}_{\psi e}^{m_{e}, m_{\psi}},
$$

such that (from 1.10 and $(1.9)$ ):

$$
a-\chi(x) \sigma_{e}^{m_{e}}(a)-\chi(\xi) \sigma_{\psi}^{m_{\psi}}(a)+\chi(x) \chi(\xi) \sigma_{\psi e}^{m_{e}, m_{\psi}}(a) \in \mathrm{SG}_{\mathrm{cl}}^{m_{e}-1, m_{\psi}-1}\left(\mathbb{R}^{d} \times \mathbb{R}^{s}\right) .
$$

We call

$$
\chi(x) \sigma_{e}^{m_{e}}(a)+\chi(\xi) \sigma_{\psi}^{m_{\psi}}(a)-\chi(x) \chi(\xi) \sigma_{\psi e}^{m_{e}, m_{\psi}}(a) \in \mathrm{SG}_{\mathrm{cl}}^{m_{e}, m_{\psi}}\left(\mathbb{R}^{d} \times \mathbb{R}^{s}\right)
$$

the principal part of $a$. Obviously it is defined only up to terms of lower order, or on principal level. On principal level, we may drop the orders from the notation and simply write

$$
\sigma(a)=\left(\sigma_{e}(a), \sigma_{\psi}(a), \sigma_{\psi e}(a)\right) .
$$

We observe that $\sigma_{e}(a)=\sigma_{\psi}(a)=0$ implies $a \in \mathrm{SG}_{\mathrm{cl}}^{m_{e}-1, m_{\psi}-1}\left(\mathbb{R}^{d} \times \mathbb{R}^{d}\right)$.

We note that a classical symbol $a$ is elliptic at $\left(x_{0}, \xi_{0}\right) \in \mathrm{W}_{\mathrm{SG}}$ if and only the corresponding component of its principal symbol is non-vanishing at $\left(x_{0}, \xi_{0}\right)$. We also note that the previous operations such as multiplication by another symbol and differentiation preserve classicality, if all involved symbols are classical. In particular, we have:

Proposition 1.18. Let $a \in \mathrm{SG}_{\mathrm{cl}}^{m_{e}, m_{\psi}}\left(\mathbb{R}^{d} \times \mathbb{R}^{d}\right)$. Then, for $\alpha, \beta \in \mathbb{N}_{0}$, we have

$$
\sigma\left(\partial_{x}^{\alpha} \partial_{\xi}^{\beta} a(x, \xi)\right)=\partial_{x}^{\alpha} \partial_{\xi}^{\beta} \sigma(a(x, \xi)),
$$

where we differentiate the right-hand side component-wise.

For a proof of this fact consider [50]. We also have a converse construction, that is from such a triple of functions we may define a corresponding "principal part", which will be an SG-amplitude that admits this triple as principal symbol:

Proposition 1.19. Let $\left(a^{e}, a^{\psi}\right)$ be a couple of functions satisfying the following assumptions: 
- $a^{e} \in \mathscr{P}_{e}^{m_{e}, m_{\psi}}$,

- $a^{\psi} \in \mathscr{P}_{\psi}^{m_{e}, m_{\psi}}$,

- $\sigma_{e}^{m_{e}}\left(a^{\psi}\right)=\sigma_{\psi}^{m_{\psi}}\left(a^{e}\right)=a^{\psi e}$.

Then, there exists $a \in \mathrm{SG}_{\mathrm{cl}}^{m_{e}, m_{\psi}}\left(\mathbb{R}^{d} \times \mathbb{R}^{d}\right)$ such that $\sigma(a)=\left(a^{e}, a^{\psi}, a^{\psi e}\right)$.

There is an additional way of characterizing classical SG-symbols and their principal symbols in terms of the isomorphism $\mathbb{R}^{d} \sqcup\left(\left(\mathbb{R}^{d} \backslash\{0\}\right) / \sim\right) \cong \mathbb{B}^{d}$ in terms of the map $\iota$ used in Construction 1.1, see [57, Chap. 8.2]:

Proposition 1.20. The map

$$
a(x, \xi) \mapsto b(y, \eta)=(1-h(y))^{m_{e}}(1-h(\eta))^{m_{\psi}}\left(\left(\iota^{-1} \times \iota^{-1}\right)^{*} a\right)(y, \eta)
$$

is an isomorphism $\iota_{\mathrm{SG}}^{m_{e}, m_{\psi}}: \mathrm{SG}_{\mathrm{cl}}^{m_{e}, m_{\psi}}\left(\mathbb{R}^{d} \times \mathbb{R}^{d}\right) \rightarrow \mathscr{C}^{\infty}\left(\mathbb{B}^{d} \times \mathbb{B}^{d}\right)$.

In light of this isomorphism, we can obtain the principal symbols of a symbol a via the restriction of $b$ to the boundary and homogeneous continuation to the interior, i.e. near the respective boundary

$$
\begin{aligned}
\left.\sigma_{\psi}^{m_{\psi}}(a)\right|_{\mathbb{R}^{d} \times \mathbb{S}^{d-1}} & =(\iota \times \mathrm{id})^{*}\left(\left.(1-h(y))^{-m_{e}} b\right|_{\widetilde{\mathrm{W}}_{\mathrm{SG}}^{\psi}}\right) \\
\left.\sigma_{e}^{m_{e}}(a)\right|_{\mathbb{S}^{d-1} \times \mathbb{R}^{d}} & =(\mathrm{id} \times \iota)^{*}\left(\left.(1-h(\eta))^{-m_{\psi}} b\right|_{\widetilde{\mathrm{W}}_{\mathrm{SG}}^{e}}\right) \\
\left.\sigma_{\psi e}^{m_{e}, m_{\psi}}(a)\right|_{\mathbb{S}^{d-1} \times \mathbb{S}^{d-1}} & =(\mathrm{id} \times \mathrm{id})^{*}\left(\left.b\right|_{\widetilde{\mathrm{W}}_{\mathrm{SG}}^{\psi e}}\right),
\end{aligned}
$$

and in particular the ellipticity of $a(x, \xi)$ at $\left(y_{0}, \eta_{0}\right) \in \widetilde{\mathrm{W}}_{\mathrm{SG}}$ - that is ellipticity at $\left(x_{0}, \xi_{0}\right) \in \mathrm{W}_{\mathrm{SG}}$ - is equivalent to the non-vanishing of $\iota_{\mathrm{SG}}^{m_{e}, m_{\psi}}(a)$ at $\left(y_{0}, \eta_{0}\right)$.

Remark 1.21. We note that the values of the restrictions of $\sigma_{\bullet}^{m \bullet}(a)$ to the above spaces (i.e. to $\mathbb{S}^{d-1} \subset \mathbb{R}^{d} \backslash\{0\}$ ) determine these, by homogeneity, on their full domain of definition.

To phrase it differently: taking the boundary defining functions $\tilde{y}=1-h(y)$ and $\tilde{\eta}=1-h(\eta)$ of Construction 1.1 (6) we have an isomorphism

$$
\mathrm{SG}_{\mathrm{cl}}^{m_{e}, m_{\psi}}\left(\mathbb{R}^{d} \times \mathbb{R}^{d}\right) \cong \tilde{y}^{-m_{e}} \tilde{\eta}^{-m_{\psi}} \mathscr{C}^{\infty}\left(\mathbb{B}^{d} \times \mathbb{B}^{d}\right) .
$$

Remark 1.22. This allows us to compare the previous setting on $\mathbb{R}^{d}$ with Melrose's scattering calculus, or sc-calculus, consider [102, Sect. 4] and [103, 104, 107]. Therein $\mathbb{B}^{d}$ is replaced by the upper half sphere $\overline{\mathbb{S}_{+}^{d}}$ and the map $\iota$ used to identify the interior with $\mathbb{R}^{d}$ is replaced by stereographic projection. The symbols are then 
introduced on the compactified space (for some boundary defining functions $\tilde{y}, \tilde{\eta}$ on the two factors)

$$
\tilde{y}^{-m_{e}} \tilde{\eta}^{-m_{\psi}} \cdot \mathscr{C}^{\infty}\left(\mathbb{S}_{+}^{d} \times \mathbb{S}_{+}^{d}\right)
$$

and pulled back to $\mathbb{R}^{d}$ using the stereographic projection, yielding precisely the classical SG-symbols.

Remark 1.23. We mention that the class of SG symbols is formally very similar to the class of bisingular symbols as introduced in [119]. Microlocal properties of this calculus are studied in [14], and therein, these similarities are investigated in more detail. 


\section{Microlocal singularities and operations}

2.1. Introduction. The (classical) wave front set $\mathrm{WF}_{\mathrm{cl}}$ on an open subset $X \subset \mathbb{R}^{d}$ was introduced by Hörmander in [77], see also [79, 81] on $T^{*} X$ as an invariant generalization of the singular support for distributions $u \in \mathscr{D}^{\prime}\left(\mathbb{R}^{d}\right)$. The wave front set encodes where a distribution fails to be "microlocally equal to a test function", in particular we have the essential feature of $\mathrm{WF}_{\mathrm{cl}}$ that

$$
\mathrm{WF}_{\mathrm{cl}}(u)=\emptyset \Leftrightarrow u \in \mathscr{C}^{\infty}\left(\mathbb{R}^{d}\right)
$$

That means $\mathrm{WF}_{\mathrm{cl}}$ encodes all types of singularities that keep a distribution from being smooth.

In this section two notions of wave front sets, $\mathrm{WF}_{\mathrm{SG}}$ and $\mathrm{WF}_{\mathrm{G}}$, suitable for the treatment of tempered distributions $u \in \mathscr{S}^{\prime}\left(\mathbb{R}^{d}\right)$ are introduced. In particular they have the global regularity property that

$$
\mathrm{WF}_{\mathrm{SG}}(u)=\emptyset \Leftrightarrow \mathrm{WF}_{\mathrm{G}}(u)=\emptyset \Leftrightarrow u \in \mathscr{S}\left(\mathbb{R}^{d}\right) .
$$

In the following we will study if and how properties and ways to characterize $\mathrm{WF}_{\mathrm{cl}}$ as well as constructions known in the classical theory of microlocal analysis can be transferred to the global setting of tempered distributions. As a first application, the two wave front sets are used to indicate microlocal existence criteria for the extension of certain operations on test functions, such as products and pairings, to tempered distributions.

Let us first provide some motivation for this: asking for the existence of the pairing of distributions is a natural question. It arises when one wants to extend operators to distributions, in particular when calculating $\langle u, A v\rangle$ for some distributions $u, v$ and an operator $A$. These expressions arise in quantum physics, scattering theory and many more constructions. The standard way of interpreting $\langle u, A v\rangle$ is via the Schwartz kernel $\mathcal{K}_{A}$ of $A$, see Theorem A.4. If $u$ and $v$ were test functions, we could write $\langle u, A v\rangle=\left\langle\mathcal{K}_{A}, u \otimes v\right\rangle$. Thus one obtains an extension of $A$ if one is able to extend the latter pairing.

A related question is that of the existence of the product of two distributions (or equivalently, by the Fourier transform and the convolution theorem (see [81, Thm 7.1.15]), of their convolution). A lot of constructions in physics involving distributions, as well as the theory of inhomogeneous differential equations, for instance Burger's equation, call for a generalization of the product of functions to 
distributions.

There is, however, a fundamental theorem by Schwartz, see [133], which can be used to state the following, 14

Theorem 2.1 (Schwartz impossibility theorem). There can be no associative multiplication $\cdot \mathscr{S}^{\prime}$ on $\mathscr{S}^{\prime}\left(\mathbb{R}^{d}\right)$ that

(1) extends the product of continuous, polynomially bounded functions, ${ }_{15}^{15}$

(2) and is compatible with the differential structure, i.e. satisfies the Leibniz rule.

One way to overcome this apparent problem in order to obtain an algebra of generalized functions is to modify the notion of product, essentially relaxing the condition (1) to only coincide with the usual product on smooth functions. For this rich field of study, with many applications, we only name some fundamental references [25, 26, 27], containing also tempered versions of the generalized functions and applications to mathematical physics.

Another approach to this problem is to find suitable generalizations of the product which do not cover all pairs $u, v \in \mathscr{S}^{\prime}\left(\mathbb{R}^{d}\right)$ but only such that satisfy certain existence criteria for the product. Following this train of thoughts, there are several ways to proceed. We confer to [87] for a survey of results known under the assumptions that

- $u$ and $v$ lie in certain "compatible spaces" 16

- $u$ and $v$ (or $\hat{u}$ and $\hat{v}$ ) have "compatible supports" 17

or a combination of the two.

In this section, we follow the microlocal approach, which has the following guiding principle:

Guiding principle. Two distributions $u$ and $v$ are microlocally compatible if there exists a family of cut-offs $\left\{\phi_{i}\right\}_{i \in I}$ that form a partition of unity such that the (cone) singular supports of $\widehat{\phi_{i} u}$ and $\widehat{\phi_{i} v}$ are "compatible".

\footnotetext{
${ }^{14}$ In the (more general) classic formulation it is assumed that the space has to contain all continuous functions. This assumption can be relaxed to fit the case of tempered distributions.

${ }^{15}$ That is if $u, v \in \mathscr{C}_{\mathrm{pol}}\left(\mathbb{R}^{d}\right)$ we have $u \cdot \mathscr{S}^{\prime} v=u \cdot \mathscr{C} v$.

${ }^{16} \mathrm{~A}$ trivial example would be that of $u \in \mathscr{S}\left(\mathbb{R}^{d}\right)$ and $v \in \mathscr{S}^{\prime}\left(\mathbb{R}^{d}\right)$.

${ }^{17}$ The trivial example being that of $u$ and $v$ with disjoint supports, yielding 0 as a product.
} 
This idea is realized in the classical Theorem [81, Thm 8.2.10] that yields the existence criterion for the product of $u, v \in \mathscr{D}^{\prime}\left(\mathbb{R}^{d}\right)$ under the assumption that $\mathrm{WF}_{\mathrm{cl}}(u) \cap \mathrm{WF}_{\mathrm{cl}}(\bar{v})=\emptyset$.

In [118, Chap. IX.10], this is reproduced in a different approach and an example is given that if $u \cdot v \in \mathscr{D}^{\prime}\left(\mathbb{R}^{d}\right)$ exists, for $u, v \in \mathscr{S}^{\prime}\left(\mathbb{R}^{d}\right)$, it is not necessarily tempered. This can be seen as follows:

Example 2.2. Consider $u=e^{i e^{x}}$ and $v=\partial_{x} \bar{u}$. Then both distributions are (distributional derivatives of) smooth bounded functions and thus tempered and we have $\mathrm{WF}_{\mathrm{cl}}(u)=\mathrm{WF}_{\mathrm{cl}}(v)=\emptyset$. However, their product can be seen to equal $-i e^{x}$, which is not tempered.

This failure to be tempered is caused by high oscillations "at infinity" of $u$ and $v$. The two kinds of wave front sets introduced in the following - associated to the SG and the G-calculus - also encode information on singularities "at infinity" caused by lack of decay and oscillations, which allows to (micro-)locally exclude such phenomena. We start with the SG-wave front set, which may be more accessible to the reader, since it is a generalization of the classical Hörmander wave front set, whereas $\mathrm{WF}_{\mathrm{G}}$ is an independent notion. 


\subsection{The SG-wave front set.}

2.2.1. Introduction, basic properties and some first examples. The SG-wave front set is a generalization of the classical wave front set of Hörmander and has been introduced under several names to study tempered distributions. ${ }^{18}$

Definition 2.3 (SG-wave front set). Let $u \in \mathscr{S}^{\prime}\left(\mathbb{R}^{d}\right)$. Then $u$ is SG-microlocally $\mathscr{S}$-regular at $\left(x_{0}, \xi_{0}\right) \in \mathrm{W}_{\mathrm{SG}}$ if there exists $A \in \mathrm{LSG}_{\mathrm{cl}}^{0,0}\left(\mathbb{R}^{d}\right)$, SG-elliptic at $\left(x_{0}, \xi_{0}\right)$, such that $A u \in \mathscr{S}\left(\mathbb{R}^{d}\right)$.

The complement $\mathrm{WF}_{\mathrm{SG}}(u)$ of all such points in $\mathrm{W}_{\mathrm{SG}}$ where $u$ is SG-microlocally $\mathscr{S}$-regular is called the SG-wave front set of $u$.

Equivalently, we may define the SG-wave front set (using Construction 1.1 and Proposition 1.20 as a subset of $\widetilde{\mathrm{W}}_{\mathrm{SG}}$, denoted by $\widetilde{\mathrm{WF}}_{\mathrm{SG}}(u)$, wherein any point in $\widetilde{\mathrm{WF}}_{\mathrm{SG}}(u)$ is in $1: 1$-correspondence to a point in $\mathrm{WF}_{\mathrm{SG}}(u)$.

In the following, we list some properties of the SG-wave front set, cf. [44]:

Lemma 2.4 (Properties of the SG-wave front set). Let $u, v \in \mathscr{S}^{\prime}\left(\mathbb{R}^{d}\right)$.

(1) Using the structure of $\widetilde{\mathrm{W}}_{\mathrm{SG}}$ and $\mathrm{W}_{\mathrm{SG}}$ respectively, we may define

$$
\begin{aligned}
\widetilde{\mathrm{WF}}_{\mathrm{SG}} & =\widetilde{\mathrm{WF}}_{\mathrm{SG}}^{\psi} \cup \widetilde{\mathrm{WF}}_{\mathrm{SG}}^{e} \cup \widetilde{\mathrm{WF}}_{\mathrm{SG}}^{\psi e} \text { and } \\
\mathrm{WF}_{\mathrm{SG}} & =\mathrm{WF}_{\mathrm{SG}}^{\psi} \cup \mathrm{WF}_{\mathrm{SG}}^{e} \cup \mathrm{WF}_{\mathrm{SG}}^{\psi e} .
\end{aligned}
$$

$\widetilde{\mathrm{WF}}_{\mathrm{SG}}(u)$ is a closed subset of $\widetilde{\mathrm{W}}_{\mathrm{SG}}$ and in particular each of the components $\mathrm{WF}_{\mathrm{SG}}^{\bullet}$ of $\mathrm{WF}_{\mathrm{SG}}$ is a closed subset of the corresponding subcomponent $\mathrm{W}_{\mathrm{SG}}^{\bullet}$. The first component coincides, under this identification, with the classical Hörmander wave front set $\mathrm{WF}_{\mathrm{cl}}(u)$, i.e.

$$
\mathrm{WF}_{\mathrm{SG}}^{e}(u)=\mathrm{WF}_{\mathrm{cl}}(u) \text {. }
$$

(2) Fourier symmetry: $(x, \xi) \in \mathrm{WF}_{\mathrm{SG}}(u) \Leftrightarrow(\xi,-x) \in \mathrm{WF}_{\mathrm{SG}}(\mathcal{F} u)$.

\footnotetext{
${ }^{18} \mathrm{~A}$ note about nomenclature: in [102, Sect. 7] the SG-wave front set appears in the context of the scattering calculus, under the name scattering-wave front set and is depicted by $\mathrm{WF}_{\mathrm{sc}}$. The equality between that definition and the one used here is checked in [44, Sect. 6]. Equivalently, in [36. Sect. 6.7.], it appears as the set ZF and is obtained via a $C^{*}$-algebraic formulation of the

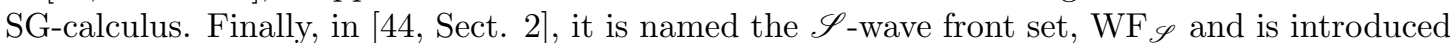
via asymptotic cut-offs and SG-operators on $\mathbb{R}^{d}$, i.e. in the same approach as taken in this document. Since both kinds of wave front sets that are studied in detail in this thesis are suited for the treatment of tempered distributions, and to avoid confusion, we are going to attach the calculus used to the respective wave front set, thus calling this one SG-wave front set.
} 
(3) $\operatorname{pr}_{1}\left(\mathrm{WF}_{\mathrm{SG}}(u)\right)=\operatorname{Css}(u), \operatorname{pr}_{2}\left(\mathrm{WF}_{\mathrm{SG}}(u)\right)=\operatorname{Css}(\mathcal{F} u)$ where $\operatorname{pr}_{i}$ denotes the projection on the $i$-th set of $d$ variables.

In particular we have global regularity: $\mathrm{WF}_{\mathrm{SG}}(u)=\emptyset \Leftrightarrow u \in \mathscr{S}\left(\mathbb{R}^{d}\right)$.

(4) Microlocality and microellipticity of SG-operators: let $A \in \mathrm{LSG}^{m_{e}, m_{\psi}}\left(\mathbb{R}^{d}\right)$. Then $\mathrm{WF}_{\mathrm{SG}}(A u) \subset \mathrm{WF}_{\mathrm{SG}}(u) \subset \mathrm{WF}_{\mathrm{SG}}(A u) \cup \operatorname{char}_{\mathrm{SG}}(A)$.

(5) $\mathrm{WF}_{\mathrm{SG}}(u+v) \subset \mathrm{WF}_{\mathrm{SG}}(u) \cup \mathrm{WF}_{\mathrm{SG}}(v)$.

We have formulated the previous properties mostly in terms of $\mathrm{WF}_{\mathrm{SG}}$. Of course, there are corresponding statements for $\widetilde{\mathrm{WF}}_{\mathrm{SG}}$. We will mainly make advantage of the formulation in terms of $\widetilde{W F}_{\mathrm{SG}}$ whenever we make statements about topological properties, such as the closedness of $\widetilde{W F}_{\mathrm{SG}}$ in the previous lemma. This will become even more useful in Section 3.2.4.

As for the classical wave front set, there exists a formulation equivalent to the one in terms of $\Psi$ DOs that uses cut-offs, essentially obtained by a local parametrix construction, that is Theorem A.23. Depending on whether $x_{0}$ and $\xi_{0}$ are points at infinity or not, corresponding cut-offs have to be used (cf. [44]):

Lemma 2.5. Let $\left(x_{0}, \xi_{0}\right)$ in $\mathrm{W}_{\mathrm{SG}}, u \in \mathscr{S}^{\prime}\left(\mathbb{R}^{d}\right)$. Then $\left(x_{0}, \xi_{0}\right) \notin \mathrm{WF}_{\mathrm{SG}}(u)$ if and only if there exist cut-offs $\phi^{x_{0}}, \phi^{\xi_{0}}$ such that

$$
\phi^{\xi_{0}} \mathcal{F}\left(\phi^{x_{0}} u\right) \in \mathscr{S}\left(\mathbb{R}^{d}\right) .
$$

Remark 2.6. Proposition A.17 yields that the notion of $\mathrm{WF}_{\mathrm{SG}}(u)$ does not depend on the choice of quantization used, since (A.17) yields that a symbol $a$ is elliptic at $\left(x_{0}, \xi_{0}\right)$ if and only if $e^{i t D_{x} D_{\xi}} a$ is elliptic there.

The following Lemma has as consequence that in the definition of $\mathrm{WF}_{\mathrm{SG}}$, the assumption on $A$ being classical may be dropped, which may also be deduced by the arguments in 42 .

Lemma 2.7. Let $A \in \mathrm{LSG}^{0,0}\left(\mathbb{R}^{d}\right)$, SG-elliptic at $\left(x_{0}, \xi_{0}\right)$, such that $A u \in \mathscr{S}\left(\mathbb{R}^{d}\right)$. Then there exists $A^{\prime} \in \mathrm{LSG}_{\mathrm{cl}}^{0,0}, \mathrm{SG}$-elliptic at $\left(x_{0}, \xi_{0}\right)$, such that $A^{\prime} u \in \mathscr{S}\left(\mathbb{R}^{d}\right)$, i.e. we can replace $A$ by a classical operator.

Proof. This is again a consequence of a localized parametrix construction, i.e. Theorem A.23:

Let $\phi^{x_{0}}, \phi^{\xi_{0}}$ cut-offs such that $\left(x_{0}, \xi_{0}\right) \in \operatorname{Csp}\left(\phi^{x_{0}} \otimes \phi^{\xi_{0}}\right)$ and $\operatorname{Csp}\left(\phi^{x_{0}} \otimes \phi^{\xi_{0}}\right) \cap$ $\left(\left(\mathbb{R}^{d} \backslash\{0\}\right) \times\left(\mathbb{R}^{d} \backslash\{0\}\right)\right) \subset \operatorname{ell}_{\mathrm{SG}}(A)$. Then we can find $B \in \mathrm{LSG}^{0,0}\left(\mathbb{R}^{d}\right)$ such that 
$B A=\left(\phi^{x_{0}} \phi^{x_{0}}\right)^{R}(x, D)+R$. Consequently we have

$$
A u \in \mathscr{S}\left(\mathbb{R}^{d}\right) \Rightarrow B A u \in \mathscr{S}\left(\mathbb{R}^{d}\right) \Rightarrow\left(\phi^{x_{0}} \otimes \phi^{\xi_{0}}\right)^{R}(x, D) u \in \mathscr{S}\left(\mathbb{R}^{d}\right) .
$$

Therefore $\phi^{x_{0}} \mathcal{F}^{-1}\left\{\phi^{\xi_{0}} \mathcal{F} u\right\} \in \mathscr{S}\left(\mathbb{R}^{d}\right)$ and thus, by Lemma 2.5. $\left(\xi_{0},-x_{0}\right)$ is not in $\mathrm{WF}_{\mathrm{SG}}(\mathcal{F} u)$, which by Lemma 2.4 is equivalent to $\left(x_{0}, \xi_{0}\right) \notin \mathrm{WF}_{\mathrm{SG}}(u)$, as claimed.

Recall that we have (with the notation of Appendix A.3.2 for the weighted Sobolev spaces)

$$
\bigcap_{\left(m_{e}, m_{\psi}\right) \in \mathbb{R}^{2}} \mathrm{H}_{\mathrm{SG}}^{m_{e}, m_{\psi}}\left(\mathbb{R}^{d}\right)=\mathscr{S}\left(\mathbb{R}^{d}\right) .
$$

It is a natural question whether one also has this equality "microlocally". This question was covered, in a more general setting, in [42, Sect. 4]. We state the particular case that is of interest here by introducing the SG-wave front set with respect to Sobolev regularity (see also [102]).

Definition 2.8 (SG-wave front set of Sobolev type). Let $u \in \mathscr{S}^{\prime}\left(\mathbb{R}^{d}\right)$. Then $u$ is $\mathrm{SG}$-microlocally $\mathrm{H}_{\mathrm{SG}}^{m_{e}, m_{\psi}}$-regular at $\left(x_{0}, \xi_{0}\right) \in \mathrm{W}_{\mathrm{SG}}$ if there exists $A \in \mathrm{LSG}_{\mathrm{cl}}^{0,0}\left(\mathbb{R}^{d}\right)$, SG-elliptic at $\left(x_{0}, \xi_{0}\right)$, such that $A u \in \mathrm{H}_{\mathrm{SG}}^{m_{e}, m_{\psi}}\left(\mathbb{R}^{d}\right)$.

The complement $\mathrm{WF}_{\mathrm{SG}}^{m_{e}, m_{\psi}}(u)$ in $\mathrm{W}_{\mathrm{SG}}$ of all such points in $\mathrm{W}_{\mathrm{SG}}$ where $u$ is $\mathrm{SG}$ microlocally $\mathrm{H}_{\mathrm{SG}}^{m_{e}, m_{\psi}}$-regular is called the $\mathrm{H}_{\mathrm{SG}}^{m_{e}, m_{\psi}}$-wave front set of $u$.

Using this definition and the results of [42, Sect. 4], we can state:

Lemma 2.9 (Sobolev resolution of $\left.\mathrm{WF}_{\mathrm{SG}}\right)$. Let $u \in \mathscr{S}^{\prime}\left(\mathbb{R}^{d}\right)$. Then

$$
\bigcap_{\left(m_{e}, m_{\psi}\right) \in \mathbb{R}^{2}} \mathrm{WF}_{\mathrm{SG}}^{m_{e}, m_{\psi}}(u)=\mathrm{WF}_{\mathrm{SG}}(u)
$$

Having established some basic properties of $\mathrm{WF}_{\mathrm{SG}}$, we proceed with some examples. The following model distributions provide an interpretation of the different components of $\mathrm{WF}_{\mathrm{SG}}$ cf. the Examples 2.5, 2.6 and (with a slight correction, see the proof below) 2.7 in [44]. 
Example 2.10. Let $x_{0} \in \mathbb{R}, \xi_{0} \in \mathbb{R}, \lambda>0$. Then we can list the SG-wave front sets of the following tempered distributions on $\mathbb{R}$ :

"Dirac delta"

"Plane wave"

"Dirac comb" $\operatorname{WF}_{\mathrm{SG}}\left(\sum_{j \in \mathbb{N}_{0}} \mathcal{T}_{\lambda j} \delta_{0}\right)=\left(\bigcup_{j \in \mathbb{N}_{0}}\{(\lambda j, \pm \infty)\}\right) \cup\{(+\infty, \pm \infty)\}$,

"Chirp"

$$
\mathrm{WF}_{\mathrm{SG}}\left(\delta_{x_{0}}\right)=\left\{\left(x_{0}, \pm \infty\right)\right\}
$$$$
\mathrm{WF}_{\mathrm{SG}}\left(e^{i \xi_{0} \cdot}\right)=\left\{\left( \pm \infty, \xi_{0}\right)\right\} \text {, }
$$

$$
\mathrm{WF}_{\mathrm{SG}}\left(e^{\frac{i}{2} \lambda(\cdot)^{2}}\right)=\{(+\infty,+\infty)\} \cup\{(-\infty,-\infty)\} \text {. }
$$

Proof. The first three examples are discussed in [44]. The fourth one needs some attention, since the claim here differs from the one in Example 2.7 in [44].

We first use Lemma 2.4 to reduce the possible wave front set of $u=e^{\frac{i}{2} \lambda x^{2}}$. First we note that $u$ is smooth, and so is $\mathcal{F} u=\sqrt{\frac{2 \pi i}{\lambda}} e^{-\frac{i}{2 \lambda}(\cdot)^{2}}$. Therefore $\mathrm{WF}_{\mathrm{SG}}^{\psi}(u)=\emptyset$ and $\mathrm{WF}_{\mathrm{SG}}^{e}(u)=\emptyset$. Since $u \notin \mathscr{S}(\mathbb{R}), \mathrm{WF}_{\mathrm{SG}}(u)$, and consequently the remaining component $\mathrm{WF}_{\mathrm{SG}}^{\psi e}(u)$, is however non-empty. Applying $\frac{1}{2 \pi} \mathcal{F}^{2} u=u$, we have $(x, \xi) \in \mathrm{WF}_{\mathrm{SG}}(u) \Leftrightarrow(-x,-\xi) \in \mathrm{WF}_{\mathrm{SG}}(u)$. It therefore remains to show that $\{(+\infty,-\infty)\} \notin \mathrm{WF}_{\mathrm{SG}}(u)$.

We prove this in terms of the characterization of $\mathrm{WF}_{\mathrm{SG}}$ using cut-offs, Lemma 2.5. Pick a mollifier ${ }^{19} \phi_{\epsilon}^{0}$ as well as a pair of asymptotic cut-offs $\phi^{ \pm \infty}$ around $\pm \infty$ supported in $[ \pm R, \infty)$ for some $R>0$ respectively. We may compute, using the continuity of the Fourier transform, in the sense of an oscillatory integral

$$
\phi^{-\infty}(\xi)\left(\mathcal{F} \phi^{+\infty} u\right)(\xi)=\lim _{\epsilon \rightarrow 0} \phi^{-\infty}(\xi) \int_{\mathbb{R}} e^{-i x \xi} \phi_{\epsilon}^{0}(x) \phi^{+\infty}(x) e^{\frac{i}{2} \lambda x^{2}} d x .
$$

For $\xi \neq x$, which is fulfilled on the support of $\phi^{+\infty} \otimes \phi^{-\infty}$, we have

$$
\underbrace{(\xi-x)^{-1} i \partial_{x}}_{=: t_{L}} e^{-i x \xi+\frac{i}{2} \lambda x^{2}}=e^{-i x \xi+\frac{i}{2} \lambda x^{2}} .
$$

Therefore, by repeated partial integration, one obtains for arbitrary $N \in \mathbb{N}_{0}$

$$
\phi^{-\infty}(\xi)\left(\mathcal{F} \phi^{+\infty} u\right)(\xi)=\lim _{\epsilon \rightarrow 0} \int_{\mathbb{R}} \phi^{-\infty}(\xi) e^{-i x \xi-\frac{i}{2} \lambda x^{2}} L^{2 N}\left(\phi_{\epsilon}^{0}(x) \phi^{+\infty}(x)\right) d x .
$$

Using the support properties of $\phi^{ \pm \infty}$, we have $|\xi-x| \gtrsim\langle\xi\rangle+\langle x\rangle$ wherever the integrand does not vanish. Thus the modulus of the integrand is bounded by

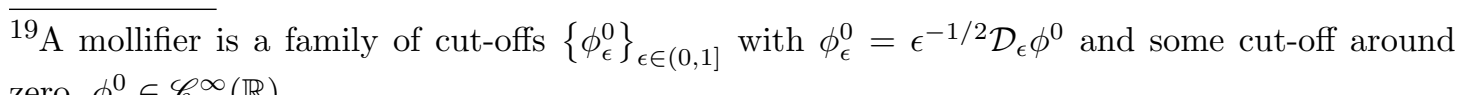
zero, $\phi^{0} \in \mathscr{C}_{c}^{\infty}(\mathbb{R})$. 
$C_{N, \epsilon}\langle\xi\rangle^{-N+d+1}\langle x\rangle^{-d-1}$, with $C_{N, \epsilon}>0$ bounded in $\epsilon$. By picking $N$ suitably large, we have arbitrary high decay in $\xi$ for $\xi \rightarrow-\infty$, which proves the claim.

The previous example attaches the following interpretations to the different components of $\mathrm{WF}_{\mathrm{SG}}(u)$, see [44]:

- $\mathrm{WF}_{\mathrm{SG}}(u) \cap\left(\mathbb{R}^{d} \times \mathbb{S}^{d-1}\right)$ encodes lack of smoothness at finite distances,

- $\mathrm{WF}_{\mathrm{SG}}(u) \cap\left(\mathbb{S}^{d-1} \times \mathbb{R}^{d}\right)$ encodes lack of decay and slow oscillations at infinity,

- $\mathrm{WF}_{\mathrm{SG}}(u) \cap\left(\mathbb{S}^{d-1} \times \mathbb{S}^{d-1}\right)$ encodes lack of smoothness and lack of decay accompanied by high oscillations at infinity.

The Dirac comb example underlines the closedness of $\mathrm{WF}_{\mathrm{SG}}(u)$ as a subset of the full wave front space $\mathrm{W}_{\mathrm{SG}}$ which corresponds to the closedness of $\widetilde{\mathrm{WF}}_{\mathrm{SG}}(u)$ in $\widetilde{\mathrm{W}}_{\mathrm{SG}}$ as in Lemma 2.4 (1). In fact, if we have a sequence of singularities in either face

$$
\mathrm{W}_{\mathrm{SG}}^{\psi}=\mathbb{R}^{d} \times\left(\left(\mathbb{R}^{d} \backslash\{0\}\right) / \sim\right) \text { or } \mathrm{W}_{\mathrm{SG}}^{e}=\left(\left(\mathbb{R}^{d} \backslash\{0\}\right) / \sim\right) \times \mathbb{R}^{d}
$$

"going to infinity" in the $\mathbb{R}^{d}$-component, there occurs a corresponding limit point in the corner component $\mathrm{WF}_{\mathrm{SG}}^{\psi e}(u)$. The example of the chirp, however, shows that not all such singularities are obtained in this manner.

2.2.2. Existence of distributions with assigned singularities. With the interpretation provided by the examples in mind, it is possible to construct a tempered distribution with any given possible wave front set, that is any closed subset of $\mathrm{W}_{\mathrm{SG}}$. Such a distribution may be constructed explicitly, which was first carried out in collaboration with Coriasco in [49], based on the classical construction in [81, Thm. 8.1.4] for $\mathrm{WF}_{\mathrm{cl}}$.

Proposition 2.11. Let $\widetilde{\Gamma}_{0} \subset \widetilde{\mathrm{W}}_{\mathrm{SG}}$ closed. Then there exists a tempered distribution $u \in \mathscr{S}^{\prime}\left(\mathbb{R}^{d}\right)$ with $\widetilde{\mathrm{WF}_{\mathrm{SG}}}(u)=\widetilde{\Gamma}_{0}$

The proof of Proposition 2.11 is divided into several steps. We first divide $\mathrm{WF}_{\mathrm{SG}}$ into its three subcomponents and then carry out the construction in each individual component. It is clear, however, that it is in general impossible to construct for instance $u^{\psi}$ such that $\widetilde{\mathrm{WF}}_{\mathrm{SG}}\left(u^{\psi}\right)=\widetilde{\mathrm{WF}}_{\mathrm{SG}}^{\psi}(u)=\widetilde{\Gamma} \cap\left(\left(\left(\mathbb{B}^{d}\right)^{o}\right) \times \mathbb{S}^{d-1}\right)$ since the latter set might not be closed in all of $\widetilde{\mathrm{W}}_{\mathrm{SG}}$. We therefore split $\widetilde{\Gamma}_{0}$ into three parts $\left(\widetilde{\Gamma}_{0}^{\psi}, \widetilde{\Gamma}_{0}^{e}, \widetilde{\Gamma}_{0}^{\psi e}\right)$ and introduce the sets $\widetilde{\Gamma}_{0}^{\bullet}$, where the closure is carried out in $\widetilde{\mathrm{W}}_{\mathrm{SG}}=\partial\left(\mathbb{B}^{d} \times \mathbb{B}^{d}\right)$. 
We obtain, using the map $\iota$ and the conification map $\Gamma$ of Construction 1.1 associated sets $\left.\Gamma_{0}=\Gamma_{0}^{\psi} \cup \Gamma_{0}^{e} \cup \Gamma_{0}^{\psi e}\right)$ in $\mathrm{W}_{\mathrm{SG}}$ and the closures

$$
\begin{array}{rlrl}
\overline{\Gamma_{0}^{\psi}} & :=\left(\iota^{-1} \times \Gamma\right)\left(\overline{\widetilde{\Gamma}_{0}^{\psi}} \cap \widetilde{\mathrm{W}}_{\mathrm{SG}}^{\psi}\right) \sqcup(\Gamma \times \Gamma)\left(\overline{\widetilde{\Gamma}_{0}^{\psi}} \cap \widetilde{\mathrm{W}}_{\mathrm{SG}}^{\psi e}\right) & & \subset \mathrm{W}_{\mathrm{SG}}^{\psi} \cup \mathrm{W}_{\mathrm{SG}}^{\psi e} \\
\overline{\Gamma_{0}^{e}}:=\left(\Gamma \times \iota^{-1}\right)\left(\overline{\widetilde{\Gamma}_{0}^{e}} \cap \widetilde{\mathrm{W}}_{\mathrm{SG}}^{e}\right) \sqcup(\Gamma \times \Gamma)\left(\overline{\widetilde{\Gamma}_{0}^{e}} \cap \widetilde{\mathrm{W}}_{\mathrm{SG}}^{\psi e}\right) & & \subset \mathrm{W}_{\mathrm{SG}}^{e} \cup \mathrm{W}_{\mathrm{SG}}^{\psi e} \\
\overline{\Gamma_{0}^{\psi e}}:=(\Gamma \times \Gamma) \overline{\widetilde{\Gamma}_{0}^{\psi e}} & & \subset \mathrm{W}_{\mathrm{SG}}^{\psi e} .
\end{array}
$$

The outline of the construction is then as follows:

(1) The classical construction is repeated (with adaptations to fit in the tempered framework) to construct a tempered distribution $u^{\psi}$ with

$$
\mathrm{WF}_{\mathrm{SG}}\left(u^{\psi}\right)=\overline{\Gamma_{0}^{\psi}} \text {. }
$$

(2) Then the Fourier symmetry of $\mathrm{WF}_{\mathrm{SG}}$ is used to obtain, by the same construction, a tempered distribution $u^{e}$ such that

$$
\mathrm{WF}_{\mathrm{SG}}\left(u^{e}\right)=\overline{\Gamma_{0}^{e}} \text {. }
$$

(3) Lastly, a tempered distribution $u^{\psi e}$ is constructed, such that

$$
\mathrm{WF}_{\mathrm{SG}}\left(u^{\psi e}\right)=\overline{\Gamma_{0}^{\psi e}}
$$

Then one defines the distribution $u=u^{\psi}+u^{e}+u^{\psi e}$, which is tempered and satisfies $\mathrm{WF}_{\mathrm{SG}}(u) \subset \Gamma_{0}$ by Lemma 2.4. The converse inclusion, in particular that no singularities can cancel, stems from the fact that $\mathrm{WF}_{\mathrm{SG}}(u)$ is a closed set. For the completion of the proof, it remains to carry out the constructions of $u^{\psi}, u^{e}$ and $u^{\psi e}$, which is achieved in a series of lemmas. The first one is essentially a version of [81, Thm. 8.1.4], with additional bounds on the coefficients, proved in [49].

Lemma 2.12 (Existence of $u^{\psi}$ ). Let $\widetilde{\Gamma}_{0}$ be closed in $\widetilde{\mathrm{W}}_{\mathrm{SG}}$. Then, there exists $u^{\psi} \in \mathscr{S}^{\prime}\left(\mathbb{R}^{d}\right)$ such that $\mathrm{WF}_{\mathrm{SG}}\left(u^{\psi}\right)=\overline{\Gamma_{0}^{\psi}}$.

Proof. Choose a dense sequence $\left(x_{k}, \xi_{k}\right) \in \Gamma_{0}$ such that $\left|\left(x_{k}, \xi_{k}\right)\right|$ is bounded by $\log k$. Let $\phi^{0} \in \mathscr{C}_{c}^{\infty}\left(\mathbb{R}^{d}\right)$ a cut-off around 0 and set

$$
u^{\psi}(x):=\sum_{k=1}^{\infty} k^{-2-d / 2} \mathcal{D}_{k} \mathcal{M}_{k^{3} \xi_{k}} \mathcal{T}_{x_{k}} \phi^{0}(x) .
$$


In fact, (2.1) is precisely the function defined in the proof of [81, Thm. 8.1.4]. The idea behind the construction is to sum up higher and higher frequency contributions around the points of singularity. In Figure 6, its representation in the timefrequency plane is sketched, for the model case where $\Gamma_{0}=\{(-1,+\infty),(1,+\infty)\}$. $u^{\psi}$ is continuous and bounded, thus it is a tempered distribution. We claim that

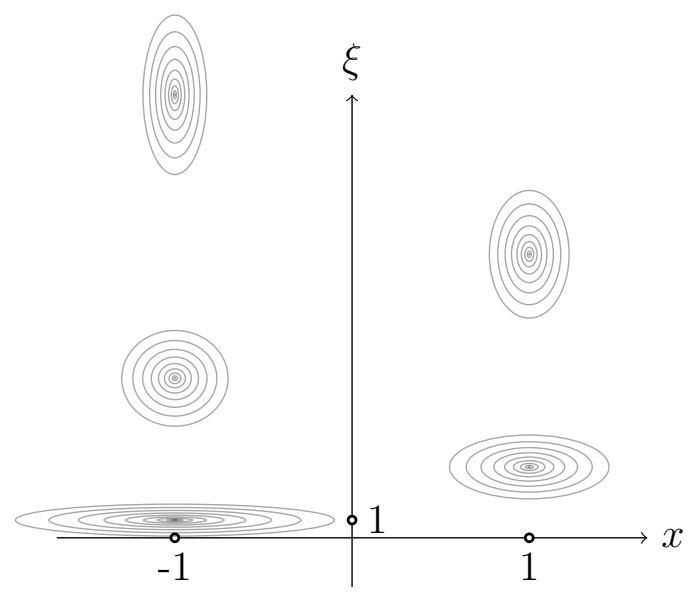

Figure 6. The construction of $u^{\psi}$, schematic

it fulfils all the required properties:

(1) $\mathrm{WF}_{\mathrm{SG}}^{\psi}\left(u^{\psi}\right)=\Gamma_{0}^{\psi}$, which is the statement of [81, Thm. 8.1.4].

(2) $\mathrm{WF}_{\mathrm{SG}}^{e}\left(u^{\psi}\right)=\emptyset$, which is, by Lemma 2.4 , equivalent to the assertion that $\widehat{u^{\psi}}$ is smooth. For that, we first note that

$$
\widehat{u^{\psi}}(\xi)=\sum_{k=1}^{\infty} k^{-2-d / 2} \mathcal{D}_{k^{-1}} \mathcal{T}_{k^{3} \xi_{k}} \mathcal{M}_{-x_{k}} \widehat{\phi^{0}} .
$$

The series 2.2 converges absolutely and uniformly, giving $\mathcal{F} u^{\psi} \in \mathscr{C}\left(\mathbb{R}^{d}\right)$, since $\widehat{\phi}^{0}$ is rapidly decaying.

We now show that $\mathcal{F} u^{\psi}$ is smooth: for any multiindex $\alpha \in \mathbb{N}_{0}^{d}$, we have

$$
\begin{aligned}
\partial_{\xi}^{\alpha} & {\left[k^{-2-d} \widehat{\phi^{0}}\left(\left(\xi-k^{3} \xi_{k}\right) / k\right) e^{i x_{k} \cdot\left(k^{3} \xi_{k}-\xi\right)}\right] \in } \\
& \in \operatorname{span}\left[k^{-2-d}\left(\partial^{\beta} \widehat{\phi^{0}}\right)\left(\left(\xi-k^{3} \xi_{k}\right) / k\right) e^{i x_{k} \cdot\left(k^{3} \xi_{k}-\xi\right)} k^{-|\beta|} x_{k}^{\gamma}\right]_{\beta+\gamma=\alpha} .
\end{aligned}
$$


Since the sequence $\left(x_{k}, \xi_{k}\right)$ is bounded in norm by $\log k$ and $(\log k)^{q} / k$, $q \in \mathbb{R}$, is a bounded sequence, it follows that the $L^{\infty}$-norm of each term in the arising sum which defines $\partial^{\alpha}\left(\mathcal{F} u^{\psi}\right)$ is bounded by the terms of the sequence

$$
C_{\alpha} \rho_{|\alpha|}^{\mathscr{S}}\left(\widehat{\phi}^{0}\right) k^{-1-d} \max _{\gamma \leq \alpha} \sup _{k}\left[k^{-1}(\log k)^{|\gamma|}\right] \leq k^{-1-d} C_{\alpha}^{\prime} \rho_{|\alpha|}^{\mathscr{S}}(\widehat{\phi}),
$$

where $C_{\alpha}, C_{\alpha}^{\prime}>0$ are suitable constants, depending only on $\alpha$. Thus $\widehat{u^{\psi}}$ yields a smooth function, as claimed, and consequently $\mathrm{WF}_{\mathrm{SG}}^{e}\left(u^{\psi}\right)=\emptyset$.

(3) $\mathrm{WF}_{\mathrm{SG}}^{\psi e}\left(u^{\psi}\right)=\overline{\Gamma_{0}^{\psi}} \cap\left(\mathbb{S}^{d-1} \times \mathbb{S}^{d-1}\right)$.

Since $\widetilde{\mathrm{WF}}_{\mathrm{SG}}\left(u^{\psi}\right)$ is a closed set in $\mathbb{B}^{d} \times \mathbb{B}^{d}$ which contains $\widetilde{\Gamma}_{0}^{e}$, the inclusion

$$
\overline{\widetilde{\Gamma}_{0}^{\psi}} \subseteq \mathrm{WF}_{\mathrm{SG}}\left(u^{\psi}\right)
$$

is immediate.

If conversely $\left(\mathbb{R}^{d} \backslash\{0\}\right) \times\left(\mathbb{R}^{d} \backslash\{0\}\right) \ni\left(x_{0}, \xi_{0}\right) \notin \overline{\Gamma_{0}^{\psi}}$, then it is possible to find admissible neighbourhoods at infinity $U, V \subset \mathbb{R}^{d}$ of $x_{0}$ and $\xi_{0}$, respectively, such that $\overline{(\iota \times \iota)(U \times V)} \cap \overline{\widetilde{\Gamma}_{0}}=\emptyset$. Choosing asymptotic cutoffs $\phi_{U}, \phi_{V}$, with supports contained in in $U$ and $V$, respectively, as in Construction 1.3 , it is possible to show, by the same reasoning as in 81 , Thm. 8.1.4], that $\psi_{V} \mathcal{F}\left\{\psi_{U} u^{\psi}\right\} \in \mathscr{S}\left(\mathbb{R}^{d}\right)$, that is, $\left(x_{0}, \xi_{0}\right) \notin \mathrm{WF}_{\mathrm{SG}}\left(u^{\psi}\right)$.

The proof is complete.

By the Fourier symmetry of $\mathrm{WF}_{\mathrm{SG}}$ as stated in Lemma 2.4, we immediately obtain the existence of $u^{e}$ :

Corollary 2.13 (Existence of $u^{e}$ ). Let $\widetilde{\Gamma}_{0}$ be closed in $\widetilde{\mathrm{W}}_{\mathrm{SG}}$. Then, there exists $u^{e} \in \mathscr{S}^{\prime}\left(\mathbb{R}^{d}\right)$ such that $\mathrm{WF}_{\mathrm{SG}}\left(u^{e}\right)=\overline{\Gamma_{0}^{e}}$.

We now turn to the construction of $u^{\psi e}$. As already mentioned, we first carried out this construction in [49] and it is repeated here with slight modifications. It relies on the same idea as in the proof of Lemma 2.12 to obtain a distribution with prescribed boundedness from below in certain directions by summing up shifted copies of the same function in the time-frequency plane. The construction is shown schematically for the case $\Gamma_{0}=\{+\infty,+\infty\}$ in Figure 7. The building block of the construction is the normalized Gaussian $\psi_{0}(x) \in \mathscr{S}\left(\mathbb{R}^{d}\right)$. 


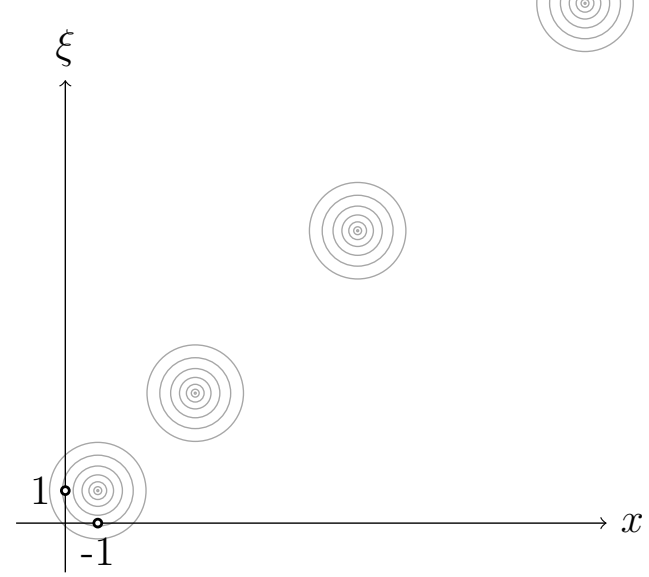

FIgURE 7. The construction of $u^{\psi e}$, schematic

Definition 2.14 (Shifted Gaussians). Let $x_{0}, \xi_{0} \in \mathbb{S}^{d-1}, k \in \mathbb{N}_{0}$. We define $\psi_{k}\left(. ; x_{0}, \xi_{0}\right) \in \mathscr{S}\left(\mathbb{R}^{d}\right)$ as

$$
\psi_{k}\left(x ; x_{0}, \xi_{0}\right):=e^{-\frac{i}{2} k^{4} x_{0} \cdot \xi_{0}} \mathcal{M}_{k^{2} \xi_{0}} \mathcal{T}_{k^{2} x_{0}} \psi_{0} .
$$

The function $\psi_{k}$ satisfies

$$
\mathcal{F}_{x \rightarrow \xi}\left\{\psi_{k}\left(x ; x_{0}, \xi_{0}\right)\right\}=(2 \pi)^{d / 2} \psi_{k}\left(\xi ; \xi_{0},-x_{0}\right) .
$$

Using the shifted Gaussians, it is possible to define a tempered distribution given by a smooth function $v\left(. ; x_{0}, \xi_{0}\right)$, which is rapidly decreasing everywhere along the ray through $x_{0}$, and whose Fourier transform is rapidly decreasing everywhere except along the ray through $\xi_{0}$.

Lemma 2.15. The series $\sum_{k=1}^{\infty} \psi_{k}\left(\cdot ; x_{0}, \xi_{0}\right)$ converges absolutely and uniformly on each compact set of $\mathbb{R}^{d}$. Its limit $v\left(\cdot ; x_{0}, \xi_{0}\right)$ is a smooth polynomially bounded function. Considering $u$ as an element of $\mathscr{S}^{\prime}\left(\mathbb{R}^{d}\right)$ we have

$$
\operatorname{Css}\left(v\left(\cdot ; x_{0}, \xi_{0}\right)\right)=\left\{x_{0} \infty\right\}
$$

The graph of $v(\cdot ; 1,1)$ is shown in Figure 8, which is fashioned after [49, Fig. 1].

Proof. The sum converges absolutely and uniformly on any compact set $K$, since for any for $k$ larger then for some $R>0$ and all $x \in K$ it holds that $e^{-\frac{1}{2}\left|x-k^{2} x_{0}\right|^{2}} \lesssim$ 


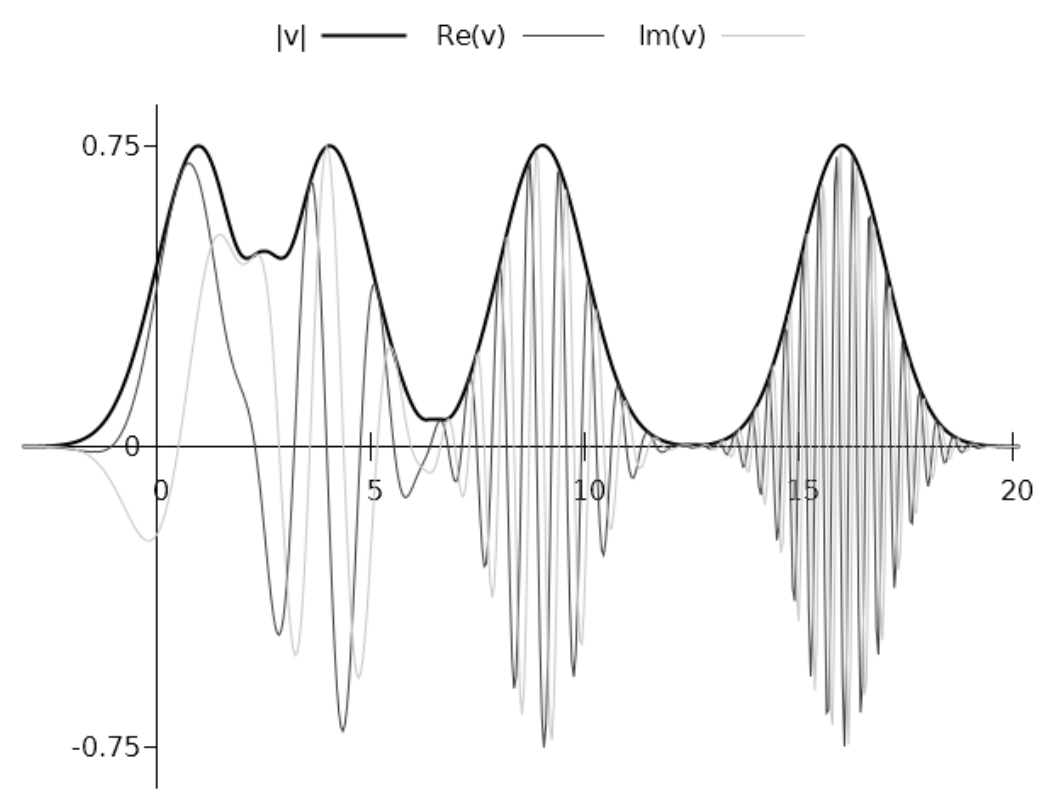

Figure 8 . The graph of $v(\cdot ; 1,1)$ (up to $k=4$ )

$e^{-\frac{1}{2} k^{4}}$ and thus

$$
\left|\sum_{k=1}^{N} \psi_{k}\left(x ; x_{0}, \xi_{0}\right)\right| \lesssim \sum_{k=1}^{N} e^{-\frac{1}{2} k^{4}} \quad x \in K .
$$

Consequently, the sum converges to a continuous function $v\left(\cdot ; x_{0}, \xi_{0}\right)$. Next, rapid decay in any other direction than that given by $x_{0}$ is shown:

Let $\Gamma \subset \mathbb{R}^{d}$ be an open cone such that $x_{0} \notin \Gamma$. By Lemma $1.4 \exists c>0$ such that for all $x \in \Gamma$ it holds that $\left|x-k^{2} x_{0}\right| \geq c\left(|x|+k^{2}\right)$.

Therefore, for arbitrary $x \in \Gamma$,

$$
\begin{aligned}
\left|x^{\alpha} v\left(x ; x_{0}, \xi_{0}\right)\right| & \leq \lim _{N \rightarrow \infty} \sum_{k=1}^{N}\left|x^{\alpha} \psi_{k}\left(x ; x_{0}, \xi_{0}\right)\right| \\
& \leq \sum_{k=1}^{N}|x|^{\alpha} \exp \left(-\frac{c}{2}\left(|x|^{2}+k^{4}\right)\right)<\infty
\end{aligned}
$$


and thus $x \infty \notin \operatorname{Css}\left(v\left(\cdot ; x_{0}, \xi_{0}\right)\right)$.

In any open cone around $x$, it holds that

$$
\left|\sum_{k=1}^{N} \psi_{k}\left(x ; x_{0}, \xi_{0}\right)\right| \leq \sum_{k=1}^{N} \exp \left(-\frac{1}{2}\left(|x|-k^{2}\right)^{2}\right),
$$

which is bounded with respect to $x$. Therefore $v\left(\cdot ; x_{0}, \xi_{0}\right)$ is bounded. Its derivatives can be estimated similarly. Consequently $v\left(\cdot ; x_{0}, \xi_{0}\right)$ is smooth and polynomially bounded in all derivatives and decreases rapidly in every direction other than that given by $x_{0}$.

Additionally, it is straight-forward to show $\left|v\left(k^{2} x_{0}, x_{0}, \xi_{0}\right)\right| \geq \frac{1}{2} \pi^{d / 4} \forall k \in \mathbb{N}_{0}$ suitably large, and therefore $x_{0} \infty \in \operatorname{Css}\left(v\left(\cdot, x_{0}, \xi_{0}\right)\right)$. This implies $v\left(\cdot ; x_{0}, \xi_{0}\right) \in$ $\left(\mathscr{C}^{\infty}\left(\mathbb{R}^{d}\right) \cap \mathscr{S}^{\prime}\left(\mathbb{R}^{d}\right)\right)$ and $\operatorname{Css}\left(v\left(\cdot ; x_{0}, \xi_{0}\right)\right)=\left\{x_{0} \infty\right\}$

Corollary 2.16. Let $v\left(x ; x_{0}, \xi_{0}\right)$ as defined in Lemma 2.15. Then,

$$
\mathrm{WF}_{\mathrm{SG}}\left(v\left(\cdot ; x_{0}, \xi_{0}\right)\right)=\left\{\left(x_{0} \infty, \xi_{0} \infty\right)\right\} \text {. }
$$

Proof. Since, by Lemma 2.4 .

$$
\begin{aligned}
& \operatorname{pr}_{1}\left(\mathrm{WF}_{\mathrm{SG}}\left(v\left(\cdot ; x_{0}, \xi_{0}\right)\right)\right)=\operatorname{Css}\left(v\left(\cdot ; x_{0}, \xi_{0}\right)\right) \\
& \operatorname{pr}_{2}\left(\mathrm{WF}_{\mathrm{SG}}\left(v\left(\cdot ; x_{0}, \xi_{0}\right)\right)\right)=\operatorname{Css}\left(\mathcal{F}\left(v\left(\cdot ; x_{0}, \xi_{0}\right)\right)\right),
\end{aligned}
$$

the claim follows from Lemma 2.15 and 2.4 .

Corollary 2.17. For any closed set $\Gamma_{0}^{\psi e} \subset \mathrm{W}_{\mathrm{SG}}^{\psi e}$, conic w.r.t. variable and covariable independently, there exists $u^{\psi e} \in \mathscr{S}^{\prime}\left(\mathbb{R}^{d}\right)$ such that $\mathrm{WF}_{\mathrm{SG}}\left(u^{\psi e}\right)=\Gamma_{0}^{\psi e}$.

Proof. Take a dense sequence without repetitions $\left\{\left(x_{l}, \xi_{l}\right)\right\}_{l \in \mathbb{N}_{0}} \subset \Gamma_{0}$ and define

$$
u^{\psi e}:=\sum_{l=0}^{\infty} 2^{-l} v\left(\cdot ; x_{l}, \xi_{l}\right) .
$$

By the properties of $v\left(\cdot ; x_{l}, \xi_{l}\right)$ and by the Weierstrass M-test, the sum (2.5) yields a smooth function that fulfils the claimed properties.

This concludes the proof of Proposition 2.11. In the construction, we have used the time-frequency plane to illustrate the position of the singularities of the involved distributions. This concept will be made precise in the next section. 


\subsection{Characterization of $\mathrm{WF}_{\mathrm{SG}}$ in the time-frequency plane.}

\subsubsection{Characterization of $\mathrm{WF}_{\mathrm{SG}}^{\psi}$ and $\mathrm{WF}_{\mathrm{SG}}^{e}$ in terms of FBI transforms.}

We have discussed that $\mathrm{WF}_{\mathrm{SG}}$ is a generalization of $\mathrm{WF}_{\mathrm{cl}}\left(=\mathrm{WF}_{\mathrm{SG}}^{\psi}\right)$. As such, it is reasonable to assume that some constructions involving $\mathrm{WF}_{\mathrm{cl}}$ may be adapted to the global setting, which we have already seen in the previous construction. We will now address that similarly to $\mathrm{WF}_{\mathrm{cl}}, \mathrm{WF}_{\mathrm{SG}}$ may be obtained in terms of a (generalized) FBI-transform.

In order to motivate this, we revisit the definition of $\mathrm{WF}_{\mathrm{SG}}^{\psi}$ using cut-offs, that is Lemma 2.5. Therein, for $\left(x_{0}, \xi_{0}\right) \in \mathbb{R}^{d} \times\left(\mathbb{R}^{d} \backslash\{0\}\right), u$ is SG-microlocally $\mathscr{S}$-regular at $\left(x_{0}, \xi_{0} \infty\right)$ if the Fourier transform of $u$, smoothly localized in an arbitrarily small open set around $x_{0}$, is rapidly decaying in a conic neighbourhood of $\xi_{0}$. This can be also restated as: there exists an open set $U \times V \ni\left(x_{0}, \xi_{0}\right)$ such that for any cut-off $\phi^{x_{0}}$ with $\operatorname{supp}\left(\phi^{x_{0}}\right) \subset U$ we have some $R>0$ such that $\forall \xi \in V$ we have

$$
\left|\left(\mathcal{F}\left\{\phi^{x_{0}} u\right\}\right)(\lambda \xi)\right|=\mathcal{O}\left(\lambda^{-\infty}\right) \quad \lambda \in[R, \infty) .
$$

The idea behind characterizing singularities in the time-frequency plane using the FBI-transform is to replace the cut-off by the normalized Gaussian standard window $\psi_{0}$. Instead of picking a small support of the localizing $\phi^{x_{0}}$ to achieve an arbitrarily fine localization, this Gaussian is rescaled and estimates in terms of the scaling parameter are used to encode microlocal regularity. We thus recall the definition (see (A.6) ) of the (global) FBI-transform of $u \in \mathscr{S}^{\prime}\left(\mathbb{R}^{d}\right)$ :

$$
\mathscr{F}_{\lambda} u(x, \xi)=C_{\psi_{0}} \mathcal{D}_{\lambda^{1 / 2}}\left(u, \mathcal{D}_{\lambda^{1 / 2}} \mathcal{M}_{\xi} \mathcal{T}_{x} \psi_{0}\right) .
$$

Studying microlocal singularities of distributions using this transform dates back to the original works of Bros and Iagolnitzer [15], consider also [37]. Such characterizations of singularities in terms of the decay properties of $\mathscr{F}_{\lambda} u$ can be achieved with respect to various function spaces (consider [23]). Since we are interested in tempered distributions and not analytical functionals here, we use the following characterization of $\mathscr{C}^{\infty}$-singularities (consider e.g. [52], [61, Chap. 3.2], [65], 155]).20

\footnotetext{
${ }^{20}$ Note that the exact conventions for the FBI-transform vary from source to source.
} 
Proposition 2.18. Let $u \in \mathscr{S}^{\prime}\left(\mathbb{R}^{d}\right)$. For some point $\left(x_{0}, \xi_{0}\right) \in \mathbb{R}^{d} \times \mathbb{S}^{d-1}$ we have $\left(x_{0}, \xi_{0} \infty\right) \notin \mathrm{WF}_{\mathrm{SG}}^{\psi}(u)$ if and only if there is an open neighbourhood $U \subset \mathbb{R}^{d} \times \mathbb{R}^{d}$ of $\left(x_{0}, \xi_{0}\right)$ for which $\exists R>0$ such that $\left|\mathscr{F}_{\lambda}(u)\right|_{U}(x, \xi) \mid=\mathcal{O}\left(\lambda^{-\infty}\right)$ for $\lambda>R$.

In the following, we will generalize this concept to the remaining components of $\mathrm{W}_{\mathrm{SG}}$. Before we present the details of this analysis, we mention that there are additional ways to characterize $\mathrm{WF}_{\mathrm{SG}}$ in the time frequency plane, see Section 4 for more details.

We can achieve a characterization of $\mathrm{WF}_{\mathrm{SG}}^{e}$ by an analogous transform using the Fourier symmetry of $\mathrm{WF}_{\mathrm{SG}}$. For that we express the FBI-transform of $\mathcal{F} u$ by that of $u$. In order to do so we introduce a generalized FBI transform, cf. also [99, 100], $\mathscr{F}_{\lambda, \mu}$ by

$$
\begin{aligned}
\mathscr{F}_{\lambda, \mu}(u)(x, \xi) & :=C_{\psi_{0}} \mathcal{D}_{(\mu \lambda)^{1 / 2}}\left(u, \mathcal{D}_{\left(\frac{\lambda}{\mu}\right)^{1 / 2}} \mathcal{T}_{x} \mathcal{M}_{\xi} \psi_{0}\right) \\
& =\left(\mathcal{D}_{\mu}\left(u * k_{\lambda, \mu, \xi}\right)\right)(x)
\end{aligned}
$$

where $k_{\lambda, \mu, \xi}$ is given by

$$
k_{\lambda, \mu, \xi}(y):=C_{\psi_{0}} \mu^{-d / 4} \lambda^{3 d / 4} \psi_{0}\left((\lambda / \mu)^{1 / 2} y\right) e^{-i \lambda y \xi}=C_{\psi_{0}} \lambda^{d / 2} \mathcal{M}_{\lambda \xi} \mathcal{D}_{\left(\frac{\lambda}{\mu}\right)^{1 / 2}} \psi_{0}(y) .
$$

In particular we can see from the first identity that $\left|\mathscr{F}_{\lambda, 1}(u)(x, \xi)\right|=\left|\mathscr{F}_{\lambda}(u)(x, \xi)\right|$. For $u=f \in \mathscr{S}\left(\mathbb{R}^{d}\right)$ this reads as

$$
\mathscr{F}_{\lambda, \mu}(f)(x, \xi)=C_{\psi_{0}} \mu^{d / 4} \lambda^{3 d / 4} \int f(y) \psi_{0}\left((\lambda / \mu)^{1 / 2}(y-\mu x)\right) e^{-i \lambda(y-\mu x) \xi} d y
$$

It can be seen as in [99] that the characterization in Proposition 2.18 does not depend on the scaling:

Lemma 2.19. Let $u \in \mathscr{S}^{\prime}\left(\mathbb{R}^{d}\right)$. For some point $\left(x_{0}, \xi_{0}\right) \in \mathbb{R}^{d} \times \mathbb{S}^{d-1}$ we have $\left(x_{0}, \xi_{0} \infty\right) \notin \mathrm{WF}_{\mathrm{SG}}^{\psi}(u)$ if and only if there exists an open neighbourhood $U \subset \mathbb{R}^{d} \times \mathbb{R}^{d}$ of $\left(\mu^{-1} x_{0}, \xi_{0}\right)$ for which $\exists R>0$ such that $\left|\mathscr{F}_{\lambda, \mu}(u)\right|_{U}(x, \xi) \mid=\mathcal{O}\left(\lambda^{-\infty}\right)$ for $\lambda>R$ and $\mu>0$ fixed.

Lemma 2.20 (Properties of the generalized FBI transform). Let $u \in \mathscr{S}^{\prime}\left(\mathbb{R}^{d}\right)$.

(1) (Continuity) $\mathscr{F}_{\lambda, \mu}(u)$ is continuous in $(x, \xi)$ and for any bounded open set $U \subset \mathbb{R}^{d} \times \mathbb{R}^{d}$ there exists $M, N \in \mathbb{N}_{0}$ such that $\left|\mathscr{F}_{\lambda, \mu}(u)\right|_{U} \mid=\mathcal{O}\left(\mu^{M} \lambda^{N}\right)$.

(2) (Fourier Symmetry) $\mathscr{F}_{\lambda, \mu}(\widehat{u})(x, \xi)=(2 \pi)^{d / 2} e^{i \lambda \mu x \xi} \mathscr{F}_{\mu, \lambda}(u)(\xi,-x)$ 
(3) (Fourier multiplier representation) We have, in the sense of tempered distributions written as integral kernels,

$$
\begin{aligned}
\left(\mathcal{F}_{x \rightarrow \eta} \mathscr{F}_{\lambda, \mu}(u)\right)(\eta, \xi) & =\left(\mathcal{D}_{\mu^{-1}}\left(\widehat{u} \cdot \widehat{k_{\lambda, \mu, \xi}}\right)\right)(\eta) \\
& =(2 \pi \lambda)^{d / 2} C_{\psi_{0}}\left(\left(\mathcal{D}_{\mu^{-1}} \widehat{u}\right) \cdot\left(\mathcal{T}_{\lambda \mu \xi} \mathcal{D}_{(\lambda \mu)^{-1 / 2}} \psi_{0}\right)\right)(\eta) .
\end{aligned}
$$

(4) (L $L^{2}$-Isometry) If $u \in L^{2}\left(\mathbb{R}^{d}\right)$, then $\mathscr{F}_{\lambda, \mu}(u) \in L^{2}\left(\mathbb{R}^{2 d}\right)$ and $\left\|\mathscr{F}_{\lambda, \mu}(u)\right\|_{2}=$ $\|u\|_{2}$.

Proof. (1) follows by applying the temperedness of $u$, i.e. $|\langle u, f\rangle| \leq \rho_{N}^{\mathscr{S}}(f)$ for some $N \in \mathbb{N}_{0}$ to A.6), see also the proof of Lemma 2.24 below.

The proof of the remaining identities is an application of the identities in Lemma A.6, the identity $\mathcal{F} \psi_{0}=(2 \pi)^{d / 2} \psi_{0}$ and $\left\|C_{\psi_{0}} \psi_{0}\right\|_{2}=(2 \pi)^{-d / 2}$ to A.6.

Corollary 2.21. Let $u \in \mathscr{S}^{\prime}\left(\mathbb{R}^{d}\right)$. For some point $\left(x_{0}, \xi_{0}\right) \in \mathbb{S}^{d-1} \times \mathbb{R}^{d}$, we have that $\left(x_{0} \infty, \xi_{0}\right) \notin \mathrm{WF}_{\mathrm{SG}}^{e}(u)$ if and only if there exists an open neighbourhood $U \subset \mathbb{R}^{d} \times \mathbb{R}^{d}$ of $\left(x_{0}, \lambda^{-1} \xi_{0}\right)$ for which $\exists R>0$ such that $\left|\mathscr{F}_{\lambda, \mu}(u)\right|_{U}(x, \xi) \mid=\mathcal{O}\left(\mu^{-\infty}\right)$ for $\mu>R$ and $\lambda>0$ fixed.

Proof. By Lemma 2.4 we have $\left(x_{0} \infty, \xi_{0}\right) \notin \mathrm{WF}_{\mathrm{SG}}(u)$ if and only if $\left(\xi_{0},-x_{0} \infty\right) \notin$ $\mathrm{WF}_{\mathrm{SG}}(\mathcal{F} u)$. By Lemma 2.19 this is equivalent to the existence of an open neighbourhood $U \subset \mathbb{R}^{d} \times \mathbb{R}^{d}$ of $\left(\mu^{-1} \xi_{0}, x_{0}\right)$ such that $\left|\mathscr{F}_{\lambda, \mu}(\mathcal{F} u)\right|_{U}(x, \xi) \mid=\mathcal{O}\left(\lambda^{-\infty}\right)$ for $\lambda>R$ for some $R>0$ and $\mu>0$ fixed. Property (2) of Lemma 2.20 yields the result.

\subsubsection{Characterization of the corner component.}

It is now only natural to look for a characterization of $\mathrm{WF}_{\mathrm{SG}}^{\psi e}$ by sending $\lambda$ and $\mu$ jointly to infinity. The main theorem of this section will be

Theorem 2.22. Let $u \in \mathscr{S}^{\prime}\left(\mathbb{R}^{d}\right),\left(x_{0}, \xi_{0}\right) \in \mathbb{S}^{d-1} \times \mathbb{S}^{d-1}$. Then $\left(x_{0} \infty, \xi_{0} \infty\right) \notin$ $\mathrm{WF}_{\mathrm{SG}}(u)$ if and only if there exists an open neighbourhood $U \times V \subset \mathbb{R}^{d} \times \mathbb{R}^{d}$ of $\left(x_{0}, \xi_{0}\right)$ such that $\left|\mathscr{F}_{\lambda, \mu} u\right|_{U \times V} \mid=\mathcal{O}\left(\lambda^{-N} \mu^{-M}\right)$ for all $N, M \in \mathbb{N}_{0}, \mu>R$ and $\lambda>R$ for some constant $R>1$.

For the implication, we proceed by proving a number of lemmas. The first one corresponds to the fact that if $u \in \mathscr{S}\left(\mathbb{R}^{d}\right)$ then $\mathrm{WF}_{\mathrm{SG}}^{\psi e}(u)=\emptyset$.

Lemma 2.23. Let $u \in \mathscr{S}\left(\mathbb{R}^{d}\right)$. Then for all $\left(x_{0}, \xi_{0}\right) \in\left(\mathbb{R}^{d} \times \mathbb{R}^{d}\right)$ with $x_{0} \neq 0$ and $\xi_{0} \neq 0$, there exists an open neighbourhood $U \times V \subset \mathbb{R}^{d} \times \mathbb{R}^{d}$ of such that 
$\left|\mathscr{F}_{\lambda, \mu} u\right|_{U \times V} \mid=\mathcal{O}\left(\lambda^{-N} \mu^{-M}\right)$ for all $N, M \in \mathbb{N}_{0}, \mu>R$ and $\lambda>R$ for some constant $R>1$.

Idea of the proof. Estimate $(2.9)$ by the use of stationary phase methods.

Our second lemma corresponds to $x_{0} \infty \notin \operatorname{Csp}(u) \Rightarrow \forall \xi \infty \in \mathbb{S}^{d-1}\left(x_{0} \infty, \xi \infty\right) \notin$ $\mathrm{WF}_{\mathrm{SG}}(u)$.

Lemma 2.24. Let $u \in \mathscr{S}^{\prime}\left(\mathbb{R}^{d}\right)$ and $x_{0} \in \mathbb{S}^{d-1}$ such that $x_{0} \infty \notin \operatorname{Csp}(u)$. Then for all $\xi_{0} \in \mathbb{R}^{d}$ with $\xi_{0} \neq 0$, there exists an open neighbourhood $U \times V \subset \mathbb{R}^{d} \times \mathbb{R}^{d}$ of $\left(x_{0}, \xi_{0}\right)$ such that $\left|\mathscr{F}_{\lambda, \mu} u\right|_{U \times V} \mid=\mathcal{O}\left(\lambda^{-N} \mu^{-M}\right)$ for all $N, M \in \mathbb{N}_{0}, \mu>R$ and $\lambda>R$ for some constant $R>1$.

Proof. Let $\tilde{\Gamma} \subset \mathbb{B}^{d}$ an open neighbourhood of $x_{0} \in \partial \mathbb{B}^{d}$ such that $\widetilde{\operatorname{Csp}}(u) \cap \overline{\tilde{\Gamma}}=\emptyset$. We then have, for some $N \in \mathbb{N}_{0}$ and $C>0$ and all $f \in \mathscr{S}\left(\mathbb{R}^{d}\right)$,

$$
|\langle u, f\rangle| \leq C \sup _{y \in \operatorname{Csp}(u)} \sum_{|\alpha|+|\beta| \leq N}\langle y\rangle^{|\alpha|}\left|\partial_{y}^{\beta} f(y)\right| .
$$

Now pick a bounded open neighbourhood $U \subset \mathbb{R}^{d}$ of $x_{0}$ open such that $\iota^{-1}(t U) \subset \Gamma$ for all $t>R>0$, some $R>0$. We thus find for $x \in U, \xi \in V$ some bounded neighbourhood of $\xi_{0}$ such that

$$
C_{\psi_{0}} \mu^{d / 4} \lambda^{3 d / 4} C \sup _{y \in \operatorname{Csp}(u)} \sum_{|\alpha|+|\beta| \leq N}\langle y\rangle^{|\alpha|}\left|\partial_{y}^{\beta}\left(\psi_{0}\left((\lambda / \mu)^{1 / 2}(y-\mu x)\right) e^{-i \lambda(y-\mu x) \xi}\right)\right| .
$$

Using Lemma 1.4 in the form $|y-\mu x|^{2} \gtrsim|y|^{2}+|\mu x|^{2}$, it is well-known how to estimate these dilated, translated and modulated Gaussians, consider i.e. [63].

By linearity of the FBI-transform and the definition of $\operatorname{Css}(u)$, we can combine the previous two lemmas into one Corollary:

Corollary 2.25. Let $u \in \mathscr{S}^{\prime}\left(\mathbb{R}^{d}\right)$ and $x_{0} \in \mathbb{S}^{d-1}$ such that $x_{0} \infty \notin \operatorname{Css}(u)$. Then for all $\xi_{0} \in \mathbb{R}^{d}$ with $\xi_{0} \neq 0$, there exists an open neighbourhood $U \times V \subset \mathbb{R}^{d} \times \mathbb{R}^{d}$ of $\left(x_{0}, \xi_{0}\right)$ such that $\left|\mathscr{F}_{\lambda, \mu} u\right|_{U \times V} \mid=\mathcal{O}\left(\lambda^{-N} \mu^{-M}\right)$ for all $N, M \in \mathbb{N}_{0}, \mu>R$ and $\lambda>R$ for some constant $R>1$.

We have now established the means to prove Theorem 2.22 
Proof of Theorem 2.22.

(" $\Rightarrow$ "): Let $\left(x_{0}, \xi_{0}\right) \in \mathbb{S}^{d-1} \times \mathbb{S}^{d-1}$ such that $\left(x_{0} \infty, \xi_{0} \infty\right) \notin \mathrm{WF}_{\mathrm{SG}}(u)$. By Lemma 2.5 we can find cut-offs $\phi^{x_{0} \infty}, \phi^{\xi_{0} \infty}$ such that $\phi^{\xi_{0} \infty} \mathcal{F}\left(\phi^{x_{0} \infty} u\right) \in \mathscr{S}\left(\mathbb{R}^{d}\right)$. But then we can find a neighbourhood $U \times V$ with the desired properties, since

$$
u=\underbrace{\left(1-\phi^{x_{0} \infty} u\right)}_{x_{0} \infty \notin \operatorname{Csp}}+\phi^{x_{0} \infty} u \text { and } \mathcal{F} \phi^{x_{0} \infty} u=\underbrace{\left(1-\phi^{\xi_{0} \infty}\right) \mathcal{F}\left(\phi^{x_{0} \infty} u\right)}_{x_{0} \infty \notin \mathrm{Csp}}+\underbrace{\phi^{\xi_{0} \infty} \mathcal{F}\left(\phi^{x_{0} \infty} u\right)}_{\in \mathscr{S}\left(\mathbb{R}^{d}\right)}
$$

such that the assertion follows by Corollary 2.25 as well as Fourier symmetry. (" $\Leftarrow$ "): Let us first calculate, $M, N \in \mathbb{N}_{0}$ and for some cut-offs $\phi^{x_{0}}$ and $\phi^{\xi_{0}}$, which are supported in $V$ and $U$ as given in the statement of the theorem, the expression $\left\langle\mathscr{F}_{\lambda, \mu}(u)(x, \cdot), \lambda^{N} \mu^{M} \phi^{x_{0}}(x) \phi^{\xi_{0}}\right\rangle_{\xi}$, meaning the pairing with respect to the $\xi$ variable. The integral is well-defined by the support properties of the cut-offs and the continuity of $\mathscr{F}_{\lambda, \mu}(u)$.

$$
\begin{gathered}
\left\langle\mathscr{F}_{\lambda, \mu}(u)(x, \cdot), \lambda^{N} \mu^{M} \phi^{x_{0}}(x) \phi^{\xi_{0}}\right\rangle_{\xi}=\lambda^{N} \mu^{M} \int \mathscr{F}_{\lambda, \mu}(u)(x, \xi) \phi^{\xi_{0}}(\xi) \phi^{x_{0}}(x) d \xi \\
=\lambda^{N} \mu^{M} \int \mathcal{F}_{\eta \rightarrow x}^{-1}\left\{\mathcal{F}_{y \rightarrow \eta} \mathscr{F}_{\lambda, \mu}(u)(y, \xi)\right\} \phi^{\xi_{0}}(\xi) \phi^{x_{0}}(x) d \xi
\end{gathered}
$$

$(\operatorname{Lemma} 2.16(2) \Rightarrow)=\lambda^{N} \mu^{M} \int \mathcal{F}_{\eta \rightarrow x}^{-1}\left(\mathcal{D}_{\mu^{-1}}\left(\widehat{u} \cdot \widehat{k_{\lambda, \mu, \xi}}\right)\right)(\eta) \phi^{\xi_{0}}(\xi) \phi^{x_{0}}(x) d \xi$

$$
\begin{aligned}
& =\lambda^{N} \mu^{M} \int \mathcal{D}_{\mu}\left(\phi^{x_{0}}\left(\mu^{-1} x\right) \mathcal{F}_{\eta \rightarrow x}^{-1}\left(\widehat{u} \cdot \widehat{k_{\lambda, \mu, \xi}}\right)(\eta)\right) \phi^{\xi_{0}}(\xi) d \xi \\
& =: \mathcal{D}_{\mu}\left(\mathcal{F}_{\eta \rightarrow x}^{-1}\left(a_{\lambda, \mu}^{N, M}(x, \eta) \widehat{u}(\eta)\right)\right)
\end{aligned}
$$

The latter expression is a (dilated) right-quantized pseudo-differential operator applied to $u$ that is given by the amplitude (by (2.8)

$$
\begin{aligned}
a_{\lambda, \mu}^{N, M}(x, \eta) & =\lambda^{N} \mu^{M} \int \phi^{x_{0}}\left(\mu^{-1} x\right) \widehat{k_{\lambda, \mu, \xi}} \phi^{\xi_{0}}(\xi) d \xi \\
& =C_{\psi_{0}} \lambda^{N} \mu^{M} \int \phi^{x_{0}}\left(\mu^{-1} x\right) \mathcal{F}\left(\lambda^{d / 2} \mathcal{M}_{-\lambda \xi} \mathcal{D}\left(\frac{\lambda}{\mu}\right)^{1 / 2} \psi_{0}\right)(\eta) \phi^{\xi_{0}}(\xi) d \xi \\
(2.13) & =(2 \pi)^{d / 2} C_{\psi_{0}} \lambda^{N-d / 2} \mu^{M} \int \phi^{x_{0}}\left(\mu^{-1} x\right) \psi_{0}\left(\left(\frac{\mu}{\lambda}\right)^{1 / 2}(\eta-\xi)\right) \phi^{\xi_{0}}\left(\lambda^{-1} \xi\right) d \xi
\end{aligned}
$$

If we now insert $\lambda=2^{l}$ and $\mu=2^{m}$, and let $m$ and $l$ suitably large, then we may sum up this expression, using the assumption

$$
\left|\left\langle\mathscr{F}_{\lambda, \mu}(u)(x, \cdot), \phi^{x_{0}}(x) \phi^{\xi_{0}}\right\rangle_{\xi}\right|=\mathcal{O}\left(\lambda^{-M} \mu^{-N}\right)
$$


for all $M, N \in \mathbb{N}_{0}$ :

$$
\begin{aligned}
\infty & >\sum_{m=R}^{\infty} \sum_{l=R}^{\infty}\left(\int\left|\left\langle\mathscr{F}_{2^{l}, 2^{m}}(u)(x, \cdot), 2^{l N} 2^{m M} \phi^{x_{0}}(x) \phi^{\xi_{0}}\right\rangle_{\xi}\right|^{2} d x\right)^{1 / 2} \\
& \stackrel{2.12}{=} \sum_{m=R}^{\infty} \sum_{l=R}^{\infty}\left(\int\left|\mathcal{D}_{2^{m}}\left(\mathcal{F}_{\eta \rightarrow x}^{-1}\left(a_{2^{l}, 2^{m}}^{N, M}(x, \eta) \widehat{u}(\eta)\right)\right)\right|^{2} d x\right)^{1 / 2} \\
& =\sum_{m=R}^{\infty} \sum_{l=R}^{\infty}\left(\int\left|\mathcal{F}_{\eta \rightarrow x}^{-1}\left(a_{2^{l}, 2^{m}}^{N, M}(x, \eta) \widehat{u}(\eta)\right)\right|^{2} d x\right)^{1 / 2} \\
& \geq\left(\int\left|\sum_{m=R}^{\infty} \sum_{l=R}^{\infty} \mathcal{F}_{\eta \rightarrow x}^{-1}\left(a_{2^{2}, 2^{m}}^{N, M}(x, \eta) \widehat{u}(\eta)\right)\right|^{2} d x\right)^{1 / 2}
\end{aligned}
$$

We are thus led to study $\sum_{\mu=R_{\mu}}^{\infty} \sum_{\lambda=R_{\lambda}}^{\infty} \mathcal{F}_{\eta \rightarrow x}^{-1}\left(a_{\lambda, \mu}^{N, M}(x, \eta) \widehat{u}(\eta)\right)$. This is a pseudodifferential operator applied to $u$ with (right) symbol (consider (2.13))

$$
\begin{aligned}
& a^{N, M}(x, \eta):= \\
& (2 \pi)^{d / 2} C_{\psi_{0}} \sum_{m=R}^{\infty} \sum_{l=R}^{\infty} 2^{l N-l d / 2+m M} \int \phi^{x_{0}}\left(\frac{x}{2^{m}}\right) \psi_{0}\left(2^{\frac{m-l}{2}}(\eta-\xi)\right) \phi^{\xi_{0}}\left(\frac{\xi}{2^{l}}\right) d \xi
\end{aligned}
$$

If we now choose $\phi^{x_{0}}$ and $\phi^{\xi_{0}}$ as in Construction 1.5, we have on the support of $\phi^{x_{0}}\left(\frac{x}{2^{m}}\right) \phi^{\xi_{0}}\left(\frac{\xi}{2^{l}}\right)$ the bound $2^{m} \asymp\langle x\rangle$ and $2^{l} \asymp\langle\xi\rangle$ jointly over all $(l, m)$. Using this and following the outline in Construction 1.5, we can estimate 2.15) from above and below (for different constants $C, c>0$ ) by

$$
\tilde{a}^{N, M}(x, \eta):=C \int\langle x\rangle^{N+d / 2}\langle\xi\rangle^{N+d / 2} \phi^{x_{0} \infty}(x) \psi_{0}\left(c\left(\frac{\langle x\rangle}{\langle\xi\rangle}\right)^{1 / 2}(\eta+\xi)\right) \phi^{\xi_{0} \infty}(\xi) d \xi
$$

for some cut-offs $\phi^{x_{0} \infty}$ and $\phi^{\xi_{0} \infty}$. We can proceed to estimate this using decay properties of the Gaussian and Lemma 1.4 to estimate $|\eta-\xi|$ in case $\eta$ and $\xi$ lie in disjoint cones to show that $a^{N, M}$ is an element of $\mathrm{SG}^{M, N-d / 2}\left(\mathbb{R}^{d} \times \mathbb{R}^{d}\right)$ and is elliptic at $\left(x_{0} \infty,-\xi_{0} \infty\right)$. For that, we may estimate the derivatives of $a^{N, M}$ in a 
similar fashion to 2.15), since, by partial integration, we have

$$
\begin{aligned}
\left|\partial_{x}^{\alpha} \partial_{\eta}^{\beta} a^{N, M}(x, \eta)\right| \lesssim \sum_{m=R}^{\infty} & \sum_{l=R}^{\infty}(-1)^{|\alpha|} 2^{l(N-d / 2-|\beta|)+m(M-|\alpha|)} \times \\
& \left|\int\left(\partial^{\alpha} \phi^{x_{0}}\right)\left(\frac{x}{2^{m}}\right) \psi_{0}\left(2^{\frac{m-l}{2}}(\eta-\xi)\right)\left(\partial^{\beta}\right) \phi^{\xi_{0}}\left(\frac{\xi}{2^{l}}\right) d \xi\right|
\end{aligned}
$$

By (2.14) we have that $a^{N, M}(x, \eta) u \in L^{2}\left(\mathbb{R}^{d}\right)$. Since $M$ and $N$ are arbitrary, we have $\left(x_{0} \infty, \xi_{0} \infty\right) \notin \mathrm{WF}_{\mathrm{SG}}(u)$, by Lemma 2.9 . 


\subsection{Operations on tempered distributions using $\mathrm{WF}_{\mathrm{SG}}$.}

\subsection{1. (Multi-)linear operations.}

So far, we have only discussed properties of the SG-wave front set and different ways to obtain the notion. As it was announced and motivated in the introduction, Section 1.2.1, we will now study a series of applications, in particular how the concept of $\mathrm{WF}_{\mathrm{SG}}$ can be used to extend operations on test functions to tempered distributions. In this, we will make use of the various characterizations of $\mathrm{WF}_{\mathrm{SG}}(u)$ (i.e. via $\Psi$ DOs, (asymptotic) cut-offs and the FBI-transform), underlining how each concept has its advantages.

In order to extend operations to distributions, we follow the principle of [82]: we first discuss elementary (multi-)linear operations on distributions, in particular tensor products, restrictions, changes of coordinates and (trivial) extensions. For these, we establish existence criteria in terms of the SG-wave front set and its propagation under such operations. Then, since every (multi-)linear map may be decomposed into such operations, we obtain a general framework to analyse the pull-back of distributions by multilinear maps.

When dealing with tempered distributions with singularities lying in some given set $\Gamma$ and studying (sequential) continuity of operations, it is often useful to use approximating sequences that "behave nicely outside $\Gamma$ ". For that, one introduces spaces of tempered distributions with prescribed wave front set and imposes a notion of convergence with respect to the singularity structure. In the following, when an operation is called sequentially continuous it is with reference to this notion of convergence.

Definition 2.26. Let $\Gamma$ a closed subset of $\mathrm{W}_{\mathrm{SG}}$. Define

$$
\mathscr{S}_{\Gamma}^{\prime}\left(\mathbb{R}^{d}\right)=\left\{u \in \mathscr{S}^{\prime}\left(\mathbb{R}^{d}\right) \mid \mathrm{WF}_{\mathrm{SG}}(u) \subset \Gamma\right\}
$$

equipped with the notion of convergence given by $u \stackrel{\mathscr{S}_{\Gamma}^{\prime}}{\longrightarrow} 0$ if

(1) $u \stackrel{\mathscr{S}^{\prime}}{\rightarrow} 0$

(2) for all $a \in \mathrm{SG}^{m_{e}, m_{\psi}}\left(\mathbb{R}^{d}\right)$ vanishing on all of $\Gamma$ we have $a(x, D) u \stackrel{\mathscr{S}}{\rightarrow} 0$.

We then have the following result, which may be achieved by carefully approximating the $e-, \psi-$ and $\psi e$-parts of a distribution seperately, using cut-offs.

Lemma 2.27. $\mathscr{S}\left(\mathbb{R}^{d}\right)$ is dense in $\mathscr{S}_{\Gamma}^{\prime}\left(\mathbb{R}^{d}\right)$. 
Remark 2.28. In this section we regard $\mathrm{WF}_{\mathrm{SG}}$ as conic set in

$$
\left(\mathbb{R}^{d} \times\left(\mathbb{R}^{d} \backslash\{0\}\right)\right) \sqcup\left(\left(\mathbb{R}^{d} \backslash\{0\}\right) \times \mathbb{R}^{d}\right) \sqcup\left(\left(\mathbb{R}^{d} \backslash\{0\}\right) \times\left(\mathbb{R}^{d} \backslash\{0\}\right)\right),
$$

allowing us to define sums in (co-)variables in $\mathbb{R}^{d} \backslash\{0\}$.

We start by examining the tensor product of tempered distributions. It is a well-known fact that the tensor product may be defined in a canonical way on $\mathscr{S}^{\prime}\left(\mathbb{R}^{d}\right) \times \mathscr{S}^{\prime}\left(\mathbb{R}^{d^{\prime}}\right)$ (consider e.g. [117, Thm. V.12]) by setting $u \otimes v$ to be the unique distribution (using nuclearity of $\mathscr{S}\left(\mathbb{R}^{d}\right)$ ) that satisfies

$$
\langle u \otimes v, f \otimes g\rangle=\langle u, f\rangle\langle v, g\rangle \quad \forall(f, g) \in \mathscr{S}\left(\mathbb{R}^{d}\right) \times \mathscr{S}\left(\mathbb{R}^{d^{\prime}}\right)
$$

It is another well-known fact ([81, Thm. 8.2.9.]), that the classical wave-front set admits a simple behaviour under tensor products: ${ }^{21}$

(2.16) $\mathrm{WF}_{\mathrm{cl}}(u \otimes v) \subset$

$$
\left(\mathrm{WF}_{\mathrm{cl}}(u) \times \mathrm{WF}_{\mathrm{cl}}(v)\right) \cup\left(\left(\mathbb{R}^{d} \times\{0\}\right) \times \mathrm{WF}_{\mathrm{cl}}(v)\right) \cup\left(\mathrm{WF}_{\mathrm{cl}}(u) \times\left(\mathbb{R}^{d^{\prime}} \times\{0\}\right)\right),
$$

where the factors are reordered according to

$$
\left(\mathbb{R}^{d} \times\left(\mathbb{R}^{d} \backslash\{0\}\right)\right) \times\left(\mathbb{R}^{d^{\prime}} \times\left(\mathbb{R}^{d^{\prime}} \backslash\{0\}\right)\right) \cong \mathbb{R}^{d+d^{\prime}} \times\left(\mathbb{R}^{d+d^{\prime}} \backslash\{0\}\right) .
$$

This can be reformulated into

Lemma 2.29. Let $u \in \mathscr{S}^{\prime}\left(\mathbb{R}^{d}\right)$ and $v \in \mathscr{S}^{\prime}\left(\mathbb{R}^{d^{\prime}}\right)$. Then $(x, \xi) \notin \mathrm{WF}_{\mathrm{SG}}^{\psi}(u)$ implies

$$
(x, y ; \xi, \eta) \notin \mathrm{WF}_{\mathrm{SG}}^{\psi}(u \otimes v) \quad \forall(y, \eta) \in \mathbb{R}^{d} \times \mathbb{R}^{d} .
$$

This can immediately be used, via Fourier symmetry of $\mathrm{WF}_{\mathrm{SG}}$, to obtain

Lemma 2.30. Let $(u, v) \in \mathscr{S}^{\prime}\left(\mathbb{R}^{d}\right) \times \mathscr{S}^{\prime}\left(\mathbb{R}^{d^{\prime}}\right)$. Then $(x, \xi) \notin \mathrm{WF}_{\mathrm{SG}}^{e}(u)$ implies

$$
(x, y ; \xi, \eta) \notin \mathrm{WF}_{\mathrm{SG}}^{e}(u \otimes v) \quad \forall(y, \eta) \in \mathbb{R}^{d} \times \mathbb{R}^{d} .
$$

The restatement in Lemma 2.29 may seem tedious at first. However, the following example shows how all the different components of the SG-wave front contribute to the corner component in the tensor product, which therefore has to be analysed carefully, and the above formulation may be used to do so.

\footnotetext{
${ }^{21}$ We neglect here the fact that it can be further reduced considering the supports of the involved distributions by the fact that $\forall u \in \mathscr{S}^{\prime}\left(\mathbb{R}^{d}\right)$ we have $x \notin \operatorname{supp}(u) \Rightarrow(x, \xi) \notin \mathrm{WF}_{\mathrm{cl}}(u) \forall \xi \in$ $\mathbb{R}^{d} \backslash\{0\}$.
} 
Example 2.31. Let $x_{0} \in \mathbb{R}$. Consider the distribution $u=\delta_{x_{0}} \in \mathscr{S}^{\prime}(\mathbb{R})$ and $v=\mathbb{1} \in \mathscr{S}^{\prime}(\mathbb{R})$. Then (recall Example 2.10) we have

$$
\begin{aligned}
& \mathrm{WF}_{\mathrm{SG}}(u)=\left\{x_{0}\right\} \times(\mathbb{R} \backslash\{0\}) \subset \mathrm{W}_{\mathrm{SG}}^{e} \\
& \mathrm{WF}_{\mathrm{SG}}(v)=(\mathbb{R} \backslash\{0\}) \times\{0\} \subset \mathrm{W}_{\mathrm{SG}}^{\psi}
\end{aligned}
$$

and

$$
\begin{aligned}
\mathrm{WF}_{\mathrm{SG}}^{\psi}(u \otimes v) & =\left\{\left(x_{0}, y ; \xi, 0\right) \mid y \in \mathbb{R}, \xi \in \mathbb{R} \backslash\{0\}\right\} \\
\mathrm{WF}_{\mathrm{SG}}^{e}(u \otimes v) & =\{(0, y ; \xi, 0) \mid y \in \mathbb{R} \backslash\{0\}, \xi \in \mathbb{R}\} \\
\mathrm{WF}_{\mathrm{SG}}^{\psi e}(u \otimes v) & =\{(0, y ; \xi, 0) \mid y \in \mathbb{R} \backslash\{0\}, \xi \in \mathbb{R} \backslash\{0\}\} .
\end{aligned}
$$

This shows that the different components of $\mathrm{WF}_{\mathrm{SG}}(u)$ and $\mathrm{WF}_{\mathrm{SG}}(v)$ mix up in $\mathrm{WF}_{\mathrm{SG}}^{\psi e}(u \otimes v)$. Obtaining a statement similar to Lemma 2.29 for the remaining component $\mathrm{WF}_{\mathrm{SG}}^{\psi e}$ is therefore more complicated, and addressing this issue using the definition of the SG-wave front set in terms of $\Psi$ DOs is technically involved. The main issue behind that is $\mathrm{SG}^{0,0}\left(\mathbb{R}^{d}\right) \otimes \mathrm{SG}^{0,0}\left(\mathbb{R}^{d^{\prime}}\right) \not \subset \mathrm{SG}^{0,0}\left(\mathbb{R}^{d+d^{\prime}}\right)$. While this may be overcome by techniques as in [82], the characterization of the corner component of $\mathrm{WF}_{\mathrm{SG}}$ via the FBI-transform in Theorem 2.22 proves to be a suitable tool to study this problem, since we have the identity

$$
\mathscr{F}_{\lambda, \mu}(u \otimes v)=\mathscr{F}_{\lambda, \mu}(u) \otimes \mathscr{F}_{\lambda, \mu}(v) .
$$

Proposition 2.32. Let $(u, v) \in \mathscr{S}^{\prime}\left(\mathbb{R}^{d}\right) \times \mathscr{S}^{\prime}\left(\mathbb{R}^{d^{\prime}}\right)$.

(1) If $(x, \xi) \notin \mathrm{WF}_{\mathrm{SG}}^{\psi e}(u)$, then

$$
(x, y ; \xi, \eta) \notin \mathrm{WF}_{\mathrm{SG}}^{\psi e}(u \otimes v) \quad \forall(y, \eta) \in \mathbb{R}^{d} \times \mathbb{R}^{d} .
$$

(2) Let $(x, \eta) \in\left(\left(\mathbb{R}^{d} \backslash\{0\}\right) \times\left(\mathbb{R}^{d^{\prime}} \backslash\{0\}\right)\right)$ and $(x, \xi) \notin \mathrm{WF}_{\mathrm{SG}}(u)$ for all $\xi \in$ $\left(\mathbb{R}^{d} \sqcup\left(\mathbb{R}^{d} \backslash\{0\}\right)\right)$ as well as $(y, \eta) \notin \mathrm{WF}_{\mathrm{SG}}(v)$ for all $y \in\left(\mathbb{R}^{d^{\prime}} \sqcup \mathbb{R}^{d^{\prime}} \backslash\{0\}\right)$. Then $(x, 0 ; 0, \eta) \notin \mathrm{WF}_{\mathrm{SG}}^{\psi e}(u \otimes v)$.

Proof. (1): This follows immediately from (2.17), Theorem 2.22 and (1) of Lemma 2.20 .

(2): The assumptions imply $x \notin \operatorname{Css}(u)$ and $\eta \notin \operatorname{Css}(\mathcal{F} v)$. Consequently, we have asymptotic cut-offs $\phi^{x}$ and $\phi^{\eta}$ s.t. $\phi^{x} u \in \mathscr{S}\left(\mathbb{R}^{d}\right)$ and $\phi^{\eta} \mathcal{F} u \in \mathscr{S}\left(\mathbb{R}^{d^{\prime}}\right)$. Consequently may find

$$
\left(1 \otimes \phi^{\eta}\right) \mathcal{F}\left\{\left(\phi^{x} \otimes 1\right)(u \otimes v)\right\}=\left(\mathcal{F} \phi^{x} u\right) \otimes\left(\phi^{\eta} \mathcal{F} v\right) \in \mathscr{S}\left(\mathbb{R}^{d+d^{\prime}}\right),
$$


which proves the claim.

Revisiting Example 2.31, we see that the map $u \mapsto u \otimes \mathbb{1}_{\mathbb{R}}$ is nothing but a generalization of the pull-back of test functions via the map $\operatorname{pr}_{d}: \mathbb{R}^{d+1} \rightarrow \mathbb{R}^{d}$ defined by $\left(x, x^{\prime}\right) \mapsto x$ to tempered distributions, since we have for $g \in \mathscr{S}\left(\mathbb{R}^{d}\right)$ and $f \in \mathscr{S}\left(\mathbb{R}^{d+1}\right)$

$$
\left\langle\operatorname{pr}_{d}^{*} g, f\right\rangle=\int\left(\operatorname{pr}_{d}^{*} g\right)\left(x, x^{\prime}\right) f\left(x, x^{\prime}\right) d x d x^{\prime}=\int g(x) f\left(x, x^{\prime}\right) d x d x^{\prime}=\left\langle g(x) \otimes \mathbb{1}_{\mathbb{R}}, f\right\rangle .
$$

We can thus establish, using the previous discussion and $\mathrm{WF}_{\mathrm{SG}}\left(\mathbb{1}_{\mathbb{R}}\right)=(\mathbb{R} \backslash\{0\}) \times$ $\{0\}$ the following lemma.

Lemma 2.33. Let $u \in \mathscr{S}^{\prime}\left(\mathbb{R}^{d}\right)$. Then we can define the pull-back of $u$ by $\mathrm{pr}_{d}$ in $\mathscr{S}^{\prime}\left(\mathbb{R}^{d+1}\right)$. We have the following inclusion:

$$
\mathrm{WF}_{\mathrm{SG}}\left(\operatorname{pr}_{d}^{*} u\right) \subset \operatorname{pr}_{d}^{*} \mathrm{WF}_{\mathrm{SG}}(u),
$$

where

$$
\begin{aligned}
& \operatorname{pr}_{d}^{*} \mathrm{WF}_{\mathrm{SG}}^{\psi}(u)=\left\{\left(x, x^{\prime}, \xi, 0\right) \mid(x, \xi) \in \mathrm{WF}_{\mathrm{SG}}^{\psi}(u), x^{\prime} \in \mathbb{R}\right\} \\
& \operatorname{pr}_{d}^{*} \mathrm{WF}_{\mathrm{SG}}^{e}(u)=\left\{\left(x, x^{\prime}, \xi, 0\right) \mid(x, \xi) \in \mathrm{WF}_{\mathrm{SG}}^{e}(u), x^{\prime} \in \mathbb{R}\right\} \cup\left\{\left(x, x^{\prime}, 0,0\right) \mid x \in \mathbb{R}^{d}, x^{\prime} \in(\mathbb{R} \backslash\{0\})\right\} \\
& \operatorname{pr}_{d}^{*} \mathrm{WF}_{\mathrm{SG}}^{\psi e}(u)=\left\{\left(x, x^{\prime}, \xi, 0\right) \mid(x, \xi) \in \mathrm{WF}_{\mathrm{SG}}^{\psi e}(u), x^{\prime} \in \mathbb{R}^{d}\right\} \\
& \cup\left\{\left(0, x^{\prime}, \xi, 0\right) \mid \exists x \in \mathbb{R}^{d} \text { s.t. }(x, \xi) \in \mathrm{WF}_{\mathrm{SG}}^{\psi}(u), x^{\prime} \in \mathbb{R} \backslash\{0\}\right\} .
\end{aligned}
$$

Remark 2.34. While the previous formulae may seem complicated at first, they are a natural extension of the classical (see e.g. [81]) formula 2.18), which can be reformulated in terms of the map ${ }^{t} \mathrm{pr}_{d}: \mathbb{R}^{d} \rightarrow \mathbb{R}^{d+1}$ given by $x \mapsto(x, 0)$ to

$$
\mathrm{WF}_{\mathrm{cl}}\left(\operatorname{pr}_{d}^{*} u\right) \subset\left\{\left(x,{ }^{t} \mathrm{pr}_{d} \xi\right) \mid\left(\operatorname{pr}_{d} x, \xi\right) \in \mathrm{WF}_{\mathrm{cl}}(u)\right\} .
$$

The behaviour in the corner component may be interpreted in the following sense: all components of the wave front sets of the factors contribute to the corner component - as in Example 2.31- and points of the form " $\left(x, x^{\prime}, \xi, \xi^{\prime} \infty\right)$ " are "condensed" into points of the form $\left(x, x^{\prime}, 0, \xi^{\prime} \infty\right)$, since $\xi$ is "infinitesimally small" compared to $\xi^{\prime} \infty .2$

\footnotetext{
${ }^{22}$ Geometrically, this corresponds to the fact that the images of parallel rays under radial compactification intersect at the boundary.
} 
Next we examine how a linear change of coordinates, i.e. the dilation operation of Lemma A.6 influence the SG-wave front set.

Lemma 2.35. Let $u \in \mathscr{S}^{\prime}\left(\mathbb{R}^{d}\right), A \in \mathrm{GL}(d, \mathbb{R})$. The $\mathrm{SG}$-wave front set of the distribution $A^{*} u:=\mathcal{D}_{A^{-1}} u \in \mathscr{S}^{\prime}\left(\mathbb{R}^{d}\right)$ fulfils

$$
\mathrm{WF}_{\mathrm{SG}}\left(A^{*} u\right)=A^{*} \mathrm{WF}_{\mathrm{SG}}(u):=\left\{\left(x,^{t} A \xi\right) \mid(A x, \xi) \in \mathrm{WF}_{\mathrm{SG}}(u)\right\} .
$$

For each $\Gamma \subset \mathrm{W}_{\mathrm{SG}}$ closed, we have that $A^{*}$ is a sequentially continuous map $\mathscr{S}_{\Gamma}^{\prime}\left(\mathbb{R}^{d}\right) \rightarrow \mathscr{S}_{A^{*} \Gamma}^{\prime}\left(\mathbb{R}^{d}\right)$.

Proof. This can be obtained using the characterization of $\mathrm{WF}_{\mathrm{SG}}$ in terms of cutoffs, Lemma 2.5 .

Remark 2.36. We could have considered more general changes of coordinates, in particular the $\mathscr{S}$-admissible coordinate transforms of [36, Chap. 4.2]. Since the focus in this document lies on the linear theory, we omit such a discussion.

We now turn to study pairings of tempered distributions using the SG-wave front set:

Theorem 2.37 (Pairings of tempered distributions via $\mathrm{WF}_{\mathrm{SG}}$ ). Let $\Gamma_{1}, \Gamma_{2} \subset \mathrm{W}_{\mathrm{SG}}$ closed subsets satisfying (in each component)

$$
(x, \xi) \in \Gamma_{1} \Rightarrow(x, \xi) \notin \Gamma_{2} .
$$

Then the map given by the pairing of two elements of $\mathscr{S}\left(\mathbb{R}^{d}\right)$ can be extended in a canonical way to $\mathscr{S}_{\Gamma_{1}}^{\prime}\left(\mathbb{R}^{d}\right) \times \mathscr{S}_{\Gamma_{2}}^{\prime}\left(\mathbb{R}^{d}\right)$. The resulting map is sequentially continuous with respect to the notions of convergence in $\mathscr{S}_{\Gamma}^{\prime}{ }^{23}$

To prove Theorem 2.37, we need to be able to choose the pseudo-differential operator used in the definition of $\mathrm{WF}_{\mathrm{SG}}$ freely, as long as the support of its (full) symbol is suitably bounded:

Lemma 2.38. Let $u \in \mathscr{S}^{\prime}\left(\mathbb{R}^{d}\right), A, B \in \mathrm{LSG}^{0,0}\left(\mathbb{R}^{d}\right)$ and let their respective symbols (modulo $\mathrm{SG}^{-\infty,-\infty}$ ) fulfil $\operatorname{Csp}(b) \subset \operatorname{ell}_{\mathrm{SG}}(a)$. Then $A u \in \mathscr{S}\left(\mathbb{R}^{d}\right)$ implies $B u \in \mathscr{S}\left(\mathbb{R}^{d}\right)$.

${ }^{23}$ That means if $\left(u_{n}, v_{n}\right) \stackrel{\mathscr{S}_{\Gamma_{1}}^{\prime} \times \mathscr{S}_{\Gamma_{2}}^{\prime}}{\longrightarrow}(u, v)$ then $\left\langle u_{n}, v_{n}\right\rangle \rightarrow\langle u, v\rangle$. 
Proof. This is a consequence of the local parametrix construction in Theorem A.23. pick $P \in \mathrm{LSG}^{0,0}$ such that $P A=B+R$ with a residual element $R: \mathscr{S}^{\prime}\left(\mathbb{R}^{d}\right) \rightarrow$ $\mathscr{S}\left(\mathbb{R}^{d}\right)$. Then $B u=P A u-R u \in \mathscr{S}\left(\mathbb{R}^{d}\right)$.

Remark 2.39. In particular, Lemma 2.38 allows us to choose a $b$ that satisfies $b \equiv 1$ in a neighbourhood of $\left(x_{0}, \xi_{0}\right) \in \operatorname{Csp}(a)$.

Proof of Theorem 2.37. Using the assumption (2.19), it is possible to use Lemma 2.38 and A.19 to construct a microlocal partition of unity:

For each point $\left(x_{i}, \xi_{i}\right) \in \mathrm{W}_{\mathrm{SG}}$ we can find an operator $A_{i} \in \mathrm{LSG}^{0,0}\left(\mathbb{R}^{d}\right)$ such that either $A_{i} u \in \mathscr{S}\left(\mathbb{R}^{d}\right)$ or ${ }^{t} A_{i} v \in \mathscr{S}\left(\mathbb{R}^{d}\right)$ and such that its symbol $a(x, \xi)$ is positive and identically one in a neighbourhood (in the sense of Construction 1.1) of $\left(x_{i}, \xi_{i}\right)$. Using the identification $\iota$ and compactness of $\widetilde{\mathrm{W}}_{\mathrm{SG}}$, we may assume a finite set $\left\{A_{i}\right\}_{i=1 . . N}$ such that $\sum_{i=1}^{N} \sigma\left(A_{i}\right)=\mathbb{1}_{\mathbb{R}^{d}}$ and $A_{i} u \in \mathscr{S}\left(\mathbb{R}^{d}\right)$ for $1 \leq i \leq k$ and ${ }^{t} A_{i} v \in \mathscr{S}\left(\mathbb{R}^{d}\right)$ for $k+1 \leq i \leq N$.

Since $\sum_{i=1}^{N} A_{i}$ is SG-elliptic, it admits a parametrix $P$ by Theorem A.21, i.e. an operator $P \in \mathrm{LSG}^{0,0}\left(\mathbb{R}^{d}\right)$ such that $P A=1+R$ with a residual element $R: \mathscr{S}^{\prime} \rightarrow$ $\mathscr{S}$. Using Lemma 2.38 once more we can conclude that even ${ }^{t} A_{i}{ }^{t} P v \in \mathscr{S}$ for $k+1 \leq i \leq N$.

We now define

$$
\langle u, v\rangle:=\sum_{i=1}^{k}\langle P \underbrace{A_{i} u}_{\in \mathscr{S}}, v\rangle+\sum_{i=k+1}^{N}\langle u, \underbrace{{ }^{t} A_{i}{ }^{t} P v}_{\in \mathscr{S}}\rangle-\langle\underbrace{R u}_{\in \mathscr{S}}, v\rangle .
$$

By a refinement argument it is straightforward to prove independence of this notion on the choice of partition of unity and the continuity statement. That the map is an extension of the product on test functions follows by approximation of $u$ and $v$ in terms of test functions as granted by Lemma 2.27 and continuity.

Lemma 2.40. Let $u \in \mathscr{S}^{\prime}\left(\mathbb{R}^{d}\right), A \in \mathrm{LSG}^{m,-\infty}\left(\mathbb{R}^{d}\right)$. Then $\mathrm{WF}_{\mathrm{SG}}^{\psi}(A u)=\emptyset$. Similarly, let $B \in \mathrm{LSG}^{-\infty, m}\left(\mathbb{R}^{d}\right)$. Then $\mathrm{WF}_{\mathrm{SG}}^{e}(A u)=\emptyset$.

Proof. Consider the case $A \in \mathrm{LSG}^{m,-\infty}\left(\mathbb{R}^{d}\right)$ and let $\left(x_{0}, \xi_{0}\right) \in \mathrm{W}_{\mathrm{SG}}^{\psi}$. Take any $B \in \operatorname{LSG}^{0,0}\left(\mathbb{R}^{d}\right)$ elliptic at $\left(x_{0}, \xi_{0}\right)$ whose symbol is compactly supported in the first set of variables, thus satisfying $B \in \mathrm{LSG}^{-\infty, 0}\left(\mathbb{R}^{d}\right)$. Then the composition $B A$ is a residual element, $B A \in \mathrm{LSG}^{-\infty,-\infty}\left(\mathbb{R}^{d}\right)$, and consequently $B(A u)=(B A) u \in$ $\mathscr{S}\left(\mathbb{R}^{d}\right)$, which proves $\left(x_{0}, \xi_{0}\right) \notin \mathrm{WF}_{\mathrm{SG}}(A u)$. 
The case where $A \in \mathrm{LSG}^{-\infty, m}\left(\mathbb{R}^{d}\right)$ follows by the same argument with exchanged variables.

Remark 2.41. As a trivial corollary we obtain the well-known statement that $u \in$ $\mathscr{S}^{\prime}\left(\mathbb{R}^{d}\right)$ and $f \in \mathscr{S}\left(\mathbb{R}^{d}\right)$ implies $u * f \in \mathscr{C}^{\infty}\left(\mathbb{R}^{d}\right) \cap \mathscr{S}^{\prime}\left(\mathbb{R}^{d}\right)$.

Proposition 2.42 (Products of tempered distributions via $\mathrm{WF}_{\mathrm{SG}}$ ). Let $\Gamma_{1}, \Gamma_{2} \subset$ $\partial\left(\mathbb{B}^{d} \times \mathbb{B}^{d}\right)$ closed subsets satisfying

$$
(x, \xi) \in\left(\Gamma_{1} \cap\left(\mathbb{B}^{d} \times \mathbb{S}^{d-1}\right)\right) \Rightarrow(x, \xi) \notin \Gamma_{2} .
$$

Then the product map $\mathscr{S}\left(\mathbb{R}^{d}\right) \times \mathscr{S}\left(\mathbb{R}^{d}\right) \rightarrow \mathscr{S}^{\prime}\left(\mathbb{R}^{d}\right)$ can be extended to a sequentially continuous map $\mathscr{S}_{\Gamma_{1}}^{\prime}\left(\mathbb{R}^{d}\right) \times \mathscr{S}_{\Gamma_{2}}^{\prime}\left(\mathbb{R}^{d}\right) \rightarrow \mathscr{S}^{\prime}\left(\mathbb{R}^{d}\right)$.

Proof. This result is a consequence of Theorem 2.37 when defining $\langle u \cdot v, f\rangle:=$ $\langle u f, v\rangle$ and using Lemma 2.40 combined with the fact that multiplication with a test function can be reformulated as the action of the $\Psi$ DO with symbol $f \otimes 1 \in$ $\mathrm{SG}^{-\infty, 0}\left(\mathbb{R}^{d} \times \mathbb{R}^{d}\right)$.

Remark 2.43. This wave front set condition for the existence of a product is far from being a necessary condition. We will discuss this in the Outlook, that is Section 4 .

Remark 2.44. The previous results about pairings and products of tempered distributions are to the best of our knowledge unpublished so far, although some have appeared in unpublished lecture notes of R.B. Melrose, [106]. Therein, the results are achieved by estimating convolutions and products directly, in particular by breaking down a general product (by use of cut-offs and Fourier transformation) into the cases $\mathscr{S}^{\prime} \cdot \mathscr{S}, \mathscr{S}^{\prime} * \mathscr{E}^{\prime}$ and $\mathscr{S}^{\prime} * \mathscr{S}$. These constructions have also been complemented by some additional details in [129]. Here we pursued a pseudodifferential approach.

Before we analyse the behaviour of the wave-front sets under such products, let us examine the case of a special product which can be used to construct an elementary pull-back. We note that this way of thinking about a pull-back has already appeared in the appendix to [118, Sect. IX.10] and the aforementioned unpublished lecture notes 106. 
Example 2.45. The distribution $v=\delta_{0} \otimes \mathbb{1}_{\mathbb{R}^{d}}$, consider Example 2.31, fulfils $\mathrm{WF}_{\mathrm{SG}}^{\psi}(v) \cup \mathrm{WF}_{\mathrm{SG}}^{\psi e}(v)=\left\{(0, y, \xi, 0) \mid y \in\left(\mathbb{R}^{d} \sqcup\left(\mathbb{R}^{d} \backslash\{0\}\right)\right), \xi \in(\mathbb{R} \backslash\{0\})\right\}$

The product of $u \in \mathscr{S}^{\prime}\left(\mathbb{R}^{d+1}\right)$ with $v$ is thus defined, in terms of Proposition 2.42 , if

$$
\mathrm{WF}_{\mathrm{SG}}(u) \cap\left\{(0, y, \xi, 0) \mid y \in\left(\mathbb{R}^{d} \sqcup\left(\mathbb{R}^{d} \backslash\{0\}\right)\right),(\xi \in \mathbb{R} \backslash\{0\})\right\}=\emptyset
$$

This product provides a way to define the pull-back of a distribution by the map $\iota_{d}: \mathbb{R}^{d} \rightarrow \mathbb{R}^{d+1}$ defined by $x \mapsto(0, x)$ :

Consider any cut-off $\phi^{0}$ around 0 and define $\iota_{d}^{*} u(f)=(u \cdot v)\left(\phi^{0} \otimes v\right)$. This map does not depend on the cut-off. We then have

$$
\left\langle u \cdot v, \phi^{0} \otimes f\right\rangle \stackrel{\frac{\text { Prop }}{2.42}}{=}\left\langle u, v \cdot\left(\phi^{0} \otimes f\right)\right\rangle=\left\langle u, v \cdot(\underbrace{\phi^{0}(0)}_{=1} \otimes f)\right\rangle,
$$

For $u=g \in \mathscr{S}\left(\mathbb{R}^{d}\right)$ this yields

$$
\left\langle g \cdot v, \phi^{0} \otimes f\right\rangle=\left\langle g, v \cdot\left(\phi^{0} \otimes f\right)\right\rangle=\left\langle g\left(\phi^{0} \otimes f\right), v\right\rangle=\langle g(0, \cdot) \cdot f, \mathbb{1}\rangle=\left\langle\iota_{d}^{*} g, f\right\rangle,
$$

so this map really extends the pull-back by $\iota_{d}^{*}$.

Now we may examine the wave-front set of the resulting distribution. This is easily done in terms of the FBI-transform, since we have for $f \in \mathscr{S}\left(\mathbb{R}^{d+1}\right)$ (with $\left(y^{\prime}, y\right)$ denoting an element in $\mathbb{R}^{d+1}$ ) after a quick calculation: ${ }^{24}$

$$
\begin{gathered}
\mathscr{F}_{\lambda, \mu}\left\{\iota_{d}^{*} f\right\}(x, \xi)=C_{\psi_{0}} \mu^{d / 4} \lambda^{3 d / 4} \int f(0, y) \psi_{0}\left(\left(\frac{\lambda}{\mu}\right)^{\frac{1}{2}}(y-\mu x)\right) e^{-i \lambda(y-\mu x) \xi} d y \\
=\int \mathscr{F}_{\lambda, \mu}(f)\left(\left(x^{\prime}, x\right),\left(\xi^{\prime}, \xi\right)\right) e^{-i \lambda \mu x^{\prime} \xi^{\prime}} d x^{\prime} d \xi^{\prime} .
\end{gathered}
$$

By approximation, this relation carries over to tempered distributions, provided the integral is defined. Using the characterization of $\mathrm{WF}_{\mathrm{SG}}$ via $\mathscr{F}_{\lambda, \mu}$, i.e. Lemma 2.19 . Corollary 2.21 and Theorem 2.22 it is thus possible to prove

Lemma 2.46. Let $u \in \mathscr{S}^{\prime}\left(\mathbb{R}^{d+1}\right)$ such that

$$
\mathrm{WF}_{\mathrm{SG}}(u) \cap\left\{(0, y, \xi, 0) \mid y \in \mathbb{R}^{d} \sqcup\left(\mathbb{R}^{d} \backslash\{0\}\right), \xi \in(\mathbb{R} \backslash\{0\})\right\}=\emptyset .
$$

Then we can define the pull-back $\iota_{d}^{*} u \in \mathscr{S}^{\prime}\left(\mathbb{R}^{d}\right)$ and we have

$$
\mathrm{WF}_{\mathrm{SG}}\left(\iota_{d}^{*} u\right) \subset \iota_{d}^{*} \mathrm{WF}_{\mathrm{SG}}(u):=\left\{(x, \xi), \mid \exists \xi^{\prime} \in \mathbb{R} \text { s.t. }\left(\iota_{d} x,\left(\xi^{\prime}, \xi\right)\right) \in \mathrm{WF}_{\mathrm{SG}}(u)\right\} .
$$

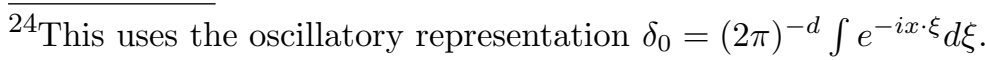


Pullbacks by general linear maps:

Since general linear maps may be decomposed into the elementary maps of the form pr, $\iota$ and a change of basis $A$, we can obtain pull-backs by general linear maps, using Lemma 2.33, 2.35 and 2.46. This follows the train of thoughts in the proof of [82, Prop. 2.9], where a similar analysis is done in the framework of the G-calculus. However, we often use different methods, in particular because the structure of the SG-wave front set makes the analysis more complicated.

This analysis may also be used to obtain a wave front inclusion for products that has (to the best of our knowledge) previously only been stated without proof in the aforementioned unpublished lecture notes [106].

Corollary 2.47. Let $u, v \in \mathscr{S}^{\prime}\left(\mathbb{R}^{d}\right)$. If the assumptions of Proposition 2.42 are fulfilled, then we have the inclusion

$$
\begin{aligned}
& \mathrm{WF}_{\mathrm{SG}}(u \cdot v) \subset \\
& \quad \mathrm{WF}_{\mathrm{SG}}(u) \cup \mathrm{WF}_{\mathrm{SG}}(v) \cup\left\{(x, \xi+\eta) \mid(x, \xi) \in \mathrm{WF}_{\mathrm{SG}}(u),(x, \eta) \in \mathrm{WF}_{\mathrm{SG}}(v)\right\}
\end{aligned}
$$

Idea of the proof. We can rewrite the product $u \cdot v=\delta^{*}(u \otimes v)$ in terms of the map $\delta: \mathbb{R}^{d} \hookrightarrow \mathbb{R}^{d} \times \mathbb{R}^{d}$ given by $\delta(x)=(x, x)$, which can be decomposed into

$$
x \stackrel{\iota_{1}}{\longmapsto}(0, x) \stackrel{x \mapsto A x}{\longmapsto}\left(\begin{array}{cc}
1 & 1 \\
-1 & 1
\end{array}\right)(0, x)=(x, x) .
$$

Using the preceding discussion on how to define general pull-backs by linear maps, we obtain the wave front set inclusion.

Remark 2.48. Proposition 2.42 and Corollary 2.47 enables us to extend convolutions to tempered distributions and estimate the resulting SG-wave front sets. This is done by using Lemma 2.4 and $f * g=\mathcal{F}^{-1}\{\mathcal{F}\{f\} \cdot \mathcal{F}\{g\}\}$. As a special case, this reproduces the wave front inclusion results known for the distributions of compact support, see e.g. [10, Prop. 3.1].

\subsubsection{Composition with operators.}

Next, we briefly discuss actions of general linear operators, following the outline in [82, Prop. 2.10.] where the analysis is carried out in the framework of the G-calculus.

Consider $A: \mathscr{S}\left(\mathbb{R}^{d}\right) \rightarrow \mathscr{S}^{\prime}\left(\mathbb{R}^{d^{\prime}}\right)$. Then, by the Schwartz kernel theorem, Theorem A.4. $A$ has a distributional kernel $\mathcal{K}_{A} \in \mathscr{S}^{\prime}\left(\mathbb{R}^{d+d^{\prime}}\right)$ defined via $\langle A f, g\rangle=$ 
$\left\langle\mathcal{K}_{A}, f \otimes g\right\rangle$. Formally, we have

$$
\begin{aligned}
A f(x) & =\int \mathcal{K}_{A}(x, y) f(y) d y \\
& =\mathcal{F}_{\xi \rightarrow x}^{-1}\left(\iota_{\xi \rightarrow(\xi, 0)}^{*} \mathcal{F}_{(x, y) \rightarrow(\xi, \eta)}\left(\mathcal{K}_{A} \cdot(\mathbb{1} \otimes f)\right)\right)
\end{aligned}
$$

The expression (2.21) is composed of the elementary maps we have already encountered:

- the tensor product by $\mathbb{1}$ (Lemma 2.33),

- the multiplication by $\mathcal{K}_{A}$ (Corollary 2.47),

- the (inverse) Fourier transformation (Lemma 2.4),

- the restriction to $\eta=0$ (Lemma 2.46).

and using these results, one may extend $A$ to tempered distributions. In particular this allows to study the propagation of singularities under partial differential equations if the distributional kernel of a solution map is known.

\subsubsection{Affine maps and translation invariant distributions.}

Having studied the composition of distributions with linear maps, we can turn to affine maps. This amounts to the study of the translation operation, which was already extended to distributions in Lemma A.6. We now state how it acts on the SG-wave front set.

Lemma 2.49. Let $u \in \mathscr{S}^{\prime}\left(\mathbb{R}^{d}\right), x_{0} \in \mathbb{R}^{d}$. Then we have

$$
\begin{aligned}
\mathrm{WF}_{\mathrm{SG}}^{\psi}\left(\mathcal{T}_{x_{0}} u\right) & =\left\{\left(x+x_{0}, \xi\right) \mid(x, \xi) \in \mathrm{WF}_{\mathrm{SG}}^{\psi}(u)\right\} \\
\mathrm{WF}_{\mathrm{SG}}\left(\mathcal{T}_{x_{0}} u\right) \backslash \mathrm{WF}_{\mathrm{SG}}^{\psi}\left(\mathcal{T}_{x_{0}} u\right) & =\mathrm{WF}_{\mathrm{SG}}(u) \backslash \mathrm{WF}_{\mathrm{SG}}^{\psi}(u) .
\end{aligned}
$$

This means that a translation by a finite element $x_{0}$ shifts $\mathrm{WF}_{\mathrm{SG}}^{\psi}(u)$, that is the differential singularities at finite distances of $u$, by $x_{0}$ in the variable and leaves the part of $\mathrm{WF}_{\mathrm{SG}}(u)$ "at infinity" invariant. We may take this analysis one step further and discuss translation invariant distributions.

Definition 2.50. Let $k \in \mathbb{N}_{0}$. A tempered distribution $u \in \mathscr{S}^{\prime}\left(\mathbb{R}^{d}\right)^{\otimes k}=\mathscr{S}^{\prime}\left(\mathbb{R}^{k d}\right)$ is translation invariant if for any $x_{0} \in \mathbb{R}^{d}$ we have $\mathcal{T}_{x_{0}}^{\otimes k} u=u$, where $\mathcal{T}_{x_{0}}^{\otimes k}$ is the map $\underbrace{\mathcal{T}_{x_{0}} \otimes \cdots \otimes \mathcal{T}_{x_{0}}}_{k \text {-fold }}$ extended to $\mathscr{S}^{\prime}\left(\mathbb{R}^{k d}\right)$.

We now analyze these kinds of tempered distributions. We first note that we have $\mathcal{F} u=\mathcal{F} \circ \mathcal{T}_{x_{0}}^{\otimes k} u=\mathcal{M}_{-x_{0}}^{\otimes k} \mathcal{F} u$, by Lemma A.6. meaning the Fourier transform 
of a translation invariant distribution is modulation invariant.

Using this, it is possible to show the following characterization of translation invariant distributions:

Lemma 2.51. Let $u \in \mathscr{S}^{\prime}\left(\mathbb{R}^{d}\right)^{\otimes k}$ translation-invariant. Then

$$
\operatorname{supp}(\widehat{u}) \subset\left\{\left(\xi_{1}, \ldots, \xi_{k}\right) \in\left(\mathbb{R}^{d}\right)^{\times k} \mid \sum_{i=1}^{k} \xi_{i}=0\right\} .
$$

There is a Theorem due to L. Schwartz, [132, Thm. 36], see also [81, Thm. 2.3.5], which characterizes these kinds of distributions supported on a subspace 25

Theorem 2.52. Let $\mathbb{R}^{d}=X \times Y$ a decomposition into subspaces. Then each distribution $u \in \mathscr{S}^{\prime}\left(\mathbb{R}^{d}\right)$ supported on $X \times\{0\}$ admits a unique decomposition into a finite sum of the form

$$
u=\sum_{|\alpha| \leq N} u_{\alpha} \otimes D_{y}^{\alpha} \delta_{Y}
$$

where $\delta_{Y}(f)=f(0)$ for all $f \in \mathscr{S}(Y), u_{\alpha} \in \mathscr{S}^{\prime}(X)$, and the $D_{y}^{\alpha_{j}}$ indicate derivatives transversal to $X \times\{0\}$.

By modulation invariance, we may even rule out the transversal derivatives in our setting and thus the Fourier transform of a translation invariant distribution is of the form $\widehat{u}=v \otimes \delta_{Y}$ where $X$ is the subspace given by the solutions to $\sum \xi_{i}=0$. This subspace is $(k-1) d$-dimensional and we can express $\delta_{Y}$ in formal notation $\delta^{d}\left(\sum_{i=1}^{k} \xi_{i}\right)$.

We can now determine the SG-wave front set of such $u$. Since $\operatorname{supp}(\widehat{u}) \subset X$, is easily seen to be contained in the set

$$
\mathrm{WF}_{\mathrm{SG}}(\widehat{u}) \subset \underbrace{X \times\left(\mathbb{R}^{d k} \backslash\{0\}\right)}_{\supset \mathrm{WF}_{\mathrm{SG}}(\widehat{u}) \cap \mathrm{W}_{\mathrm{SG}}^{\psi}} \sqcup \underbrace{(X \backslash\{0\}) \times\left(\mathbb{R}^{d k} \backslash\{0\}\right)}_{\supset \mathrm{WF}_{\mathrm{SG}}(\widehat{u}) \cap \mathrm{W}_{\mathrm{SG}}^{\psi e}} \sqcup \underbrace{(X \backslash\{0\}) \times \mathbb{R}^{d k}}_{\supset \mathrm{WF}_{\mathrm{SG}}(\widehat{u}) \cap \mathrm{W}_{\mathrm{SG}}^{e}}
$$

By the Fourier symmetry of $\mathrm{WF}_{\mathrm{SG}}$, that is Lemma 2.4, we thus have for translation invariant distributions

$$
\mathrm{WF}_{\mathrm{SG}}(u) \subset \underbrace{\mathbb{R}^{d k} \times(X \backslash\{0\})}_{\supset \mathrm{WF}_{\mathrm{SG}}(u) \cap \mathrm{W}_{\mathrm{SG}}^{\psi}} \sqcup \underbrace{\left(\mathbb{R}^{d k} \backslash\{0\}\right) \times(X \backslash\{0\})}_{\supset \mathrm{WF}_{\mathrm{SG}}(u) \cap \mathrm{W}_{\mathrm{SG}}^{\psi e}} \sqcup \underbrace{\left(\mathbb{R}^{d k} \backslash\{0\}\right) \times X}_{\supset \mathrm{WF}_{\mathrm{SG}}(u) \cap \mathrm{W}_{\mathrm{SG}}^{e}}
$$

We deduce from Proposition 2.42 .

\footnotetext{
${ }^{25}$ We state a tempered version of the Theorem, which follows trivially from the original one.
} 
Corollary 2.53. Let $u \in \mathscr{S}^{\prime}\left(\mathbb{R}^{d k}\right)$ translation invariant, $v \in \mathscr{S}^{\prime}\left(\mathbb{R}^{d k}\right)$ such that

$$
(x, \xi) \in\left(\mathrm{WF}_{\mathrm{SG}}^{\psi}(v) \cup \mathrm{WF}_{\mathrm{SG}}^{\psi e}\right) \Rightarrow \sum_{k} \xi_{k} \neq 0 .
$$

Then $u \cdot v$ yields a well-defined tempered distribution.

In particular Condition 2.22 is met if $\operatorname{pr}_{2}\left(\mathrm{WF}_{\mathrm{SG}}(v) \cap\left(\mathrm{W}_{\mathrm{SG}}^{\psi} \cup \mathrm{W}_{\mathrm{SG}}^{\psi e}\right)\right)=\Gamma$ for some convex cone $\Gamma \subset \mathbb{R}^{d k} \backslash\{0\}$.

Remark 2.54 (A remark about applicability in quantum field theory). This corollary can be used to give a simple proof of the "Theorem 0" of Epstein and Glaser [58] by microlocal methods. A microlocal proof of this in terms of $\mathscr{D}^{\prime}(X)$ (on manifolds $X$ ) has already been given in [16]. Here, however, we also take the temperedness of the involved distributions into the account, as in the original formulation of the result.

In Section 3.5, we will study several other applications of these global methods to quantum field theory.

Having established some first applications of $\mathrm{WF}_{\mathrm{SG}}$, we now turn to a different global notion of singularities on $\mathbb{R}^{d}$, associated to the $G$-calculus and the compactification of $T^{*} \mathbb{R}^{d}$ by $\mathrm{W}_{\mathrm{G}}$. 


\subsection{The G-wave front set.}

\subsubsection{Introduction, basic properties and some first examples.}

A second, maybe lesser known, wave front set suitable for the analysis of tempered distributions on $\mathbb{R}^{d}$ is the G-wave front set. It was originally defined by Hörmander [82] to study propagation of singularities for quadratic hyperbolic operators. Recently, the notion has attracted attention due to its applicability to Schrödinger equations and (almost) metaplectic operators, see e.g. [30, 34].

In the following, we will point out several common features and differences between the two notions $\mathrm{WF}_{\mathrm{SG}}$ and $\mathrm{WF}_{\mathrm{G}}$ and other wave front sets. In particular, we already mention that in a recent approach, Nakamura defined a similar notion called homogeneous wave front set, $\operatorname{HWF}(u)$, in [111] by methods of semi-classical analysis, see also [100, 101, 109]. The equality $\operatorname{HWF}(u)=\mathrm{WF}_{\mathrm{G}}(u)$ and the relation between $\mathrm{WF}_{\mathrm{G}}$ and other notions of wave front set will be discussed in more detail in Section 2.6 ${ }^{26}$ For now we mention that the main difference with respect to the SG-wave front set (or in that matter the classical one) is that in $\mathrm{WF}_{\mathrm{G}}$ any point outside $\{0\} \times\left(\mathbb{R}^{d} \backslash\{0\}\right)$ encodes both high frequency contributions as well as large spacial arguments. This may be interpreted from a geometrical viewpoint by considering the different compactification and of $T^{*} \mathbb{R}^{d}$ used, i.e. $\mathbb{B}^{2 d}$ as opposed to $\mathbb{B}^{d} \times \mathbb{B}^{d}$, and the different induced notion of neighbourhoods at infinity, as shown in Figure 5 .

We begin by introducing $\mathrm{WF}_{\mathrm{G}}$ using Weyl-quantized pseudodifferential operators with Shubin symbols. The following definition and basic properties are due to [82].

Definition 2.55 (G-wave front set). Let $u \in \mathscr{S}^{\prime}\left(\mathbb{R}^{d}\right)$. Then $u$ is G-microlocally $\mathscr{S}$-regular at $\left(x_{0}, \xi_{0}\right) \in \mathrm{W}_{\mathrm{G}}$ if there exists $a \in \mathrm{G}^{0}\left(\mathbb{R}^{d} \times \mathbb{R}^{d}\right)$, G-elliptic at $\left(x_{0}, \xi_{0}\right)$, such that $a^{W}(x, D) u \in \mathscr{S}\left(\mathbb{R}^{d}\right)$.

The complement $\mathrm{WF}_{\mathrm{G}}(u)$ in $\mathrm{W}_{\mathrm{G}}$ of all such points at which $u$ is G-microlocally $\mathscr{S}$-regular is called the G-wave front set of $u$.

Lemma 2.56. Let $u \in \mathscr{S}^{\prime}\left(\mathbb{R}^{d}\right)$.

\footnotetext{
${ }^{26} \mathrm{~A}$ note about nomenclature: In $\left[22\right.$, Hörmander simply calls this notion $\mathscr{C}^{\infty}$-wave front set, WF, thus not distinguishing from the classical wave front set. Nakamura 111 uses the symbol HWF for his homogeneous wave front set. In the recent [121, Hörmander's original object is called Gabor wave front set, $\mathrm{WF}_{\mathrm{G}}$, due to the newly found characterization in terms of Gabor frames. In this document, we also use the symbol $\mathrm{WF}_{\mathrm{G}}$, but simply call it G-wave front set to emphasize the calculus used.
} 
(1) $\mathrm{WF}_{\mathrm{G}}(u)$ is a closed conic (in both variable and covariable jointly) subset of $\mathrm{W}_{\mathrm{G}}=\left(\mathbb{R}^{2 d} \backslash\{0\}\right)$.

(2) Microlocality of G-operators: let $A \in \mathrm{LG}^{m}\left(\mathbb{R}^{d}\right)$. Then $\mathrm{WF}_{\mathrm{G}}(A u) \subset$ $\mathrm{WF}_{\mathrm{G}}(u)$.

(3) Global regularity: $\mathrm{WF}_{\mathrm{G}}(u)=\emptyset \Leftrightarrow u \in \mathscr{S}\left(\mathbb{R}^{d}\right)$.

Remark 2.57. We may again introduce the analogous object $\widetilde{\mathrm{WF}}_{G}$ as a subset of $\widetilde{\mathrm{W}}_{\mathrm{G}}=\mathbb{S}^{2 d-1}$ by identifying $\left(x_{0}, \xi_{0}\right)$ with the ray $\mathbb{R}_{+} \cdot\left(x_{0}, \xi_{0}\right)$. We will not make much use of this, however. The reason to use $\widetilde{W}_{\mathrm{SG}}$ in the SG-context is that therein, statements about topological properties "at infinity" are easily formulated. In the G-setting, the topological structure is much simpler (since there is no corner component), allowing us to simply work in $\mathrm{W}_{\mathrm{G}}$.

We also note that it is straight-forward to introduce Sobolev-analogons of $\mathrm{WF}_{\mathrm{G}}$ as in Definition 2.8 for the SG setting, see also [111].

The following proposition underlines one of the main features of $\mathrm{WF}_{\mathrm{G}}$, its behaviour under symplectic transformations.

Proposition 2.58 (Behaviour of $\mathrm{WF}_{\mathrm{G}}$ under metaplectic transformations). Recall that for each linear symplectic map $\chi$ in $T^{*} \mathbb{R}^{d}$, there exists a (unique up to a phase factor) unitary operator $U_{\chi}$ on $L^{2}\left(\mathbb{R}^{d}\right)$, such that

$$
U_{\chi}^{*} a^{W}(x, D) U_{\chi}=(a \circ \chi)^{W}(x, D) .
$$

Let $u \in \mathscr{S}^{\prime}\left(\mathbb{R}^{d}\right)$ and let $\chi$ be a linear symplectic map. Then

$$
\mathrm{WF}_{\mathrm{G}}\left(U_{\chi} u\right)=\left\{\chi(x, \xi) \mid(x, \xi) \in \mathrm{WF}_{\mathrm{G}}(u)\right\} .
$$

In particular we have for the generators of such linear symplectic transformations

- Fourier symmetry: Consider for some $k \in \mathbb{N}_{0}$ with $0<k<d$ the splitting $T^{*} \mathbb{R}^{d} \cong T^{*}\left(\mathbb{R}^{d-k} \times \mathbb{R}^{k}\right)$ and denote its elements by $(x, y ; \xi, \eta)$. Then we have Fourier Symmetry:

$$
(x, y ; \xi, \eta) \in \mathrm{WF}_{\mathrm{G}}(u) \Leftrightarrow(\xi, y ;-x, \eta) \in \mathrm{WF}_{\mathrm{G}}\left(\mathcal{F}_{x \rightarrow \xi} u\right) .
$$

- Covariance: Let $A \in \mathrm{GL}(d, \mathbb{R})$. Then

$$
(x, \xi) \in \mathrm{WF}_{\mathrm{G}}(u) \Leftrightarrow\left(A^{-1} x,{ }^{t} A \xi\right) \in \mathrm{WF}_{\mathrm{G}}\left(\mathcal{D}_{A} u\right) .
$$

\footnotetext{
${ }^{27} \mathrm{~A}$ proof for this fact may be found in [83, Thm. 18.5.9.], see also [82] and [94, Thm. 2.1.2.].
} 
- Multiplication by a chirp: Let $A$ a real, symmetric $d \times d$ matrix. Then

$$
(x, \xi) \in \mathrm{WF}_{\mathrm{G}}(u) \Leftrightarrow(x, \xi+A x) \in \mathrm{WF}_{\mathrm{G}}\left(e^{\frac{i}{2}\langle A x, x\rangle} u\right) .
$$

We can now review the distributions of Example 2.10 in the context of the G-wave front set:

Example 2.59. Let $x_{0} \in \mathbb{R}, \xi_{0} \in \mathbb{R}, \lambda>0$. Then we can list the G-wave front sets of the following tempered distributions on $\mathbb{R}$, cf. [121]:

$$
\begin{aligned}
\mathrm{WF}_{\mathrm{G}}\left(\delta_{x_{0}}\right) & =\{0\} \times(\mathbb{R} \backslash\{0\}), \\
\mathrm{WF}_{\mathrm{G}}\left(e^{i \xi_{0} \cdot}\right) & =(\mathbb{R} \backslash\{0\}) \times\{0\}, \\
\mathrm{WF}_{\mathrm{G}}\left(\sum_{j \in \mathbb{N}_{0}} \mathcal{T}_{\lambda j} \delta_{0}\right) & =\left\{(x, y) \in\left(\mathbb{R}^{2} \backslash\{0\}\right) \mid x \geq 0\right\}, \\
\mathrm{WF}_{\mathrm{G}}\left(e^{\frac{i}{2} \lambda(\cdot)^{2}}\right) & =\left(\mathbb{R}_{+} \cdot\{(1, \lambda)\}\right) \cup\left(\mathbb{R}_{+} \cdot\{(-1,-\lambda)\}\right) .
\end{aligned}
$$

Therefore the information about lack of smoothness at finite points is condensed into $\{0\} \times \mathbb{R} \backslash\{0\}$, lack of decay and slow oscillations at infinity are encoded at $\mathbb{R} \backslash\{0\} \times\{0\}$ and the rest of $\mathbb{R}^{2} \backslash\{0\}$ encodes higher oscillations, in particular quadratic ones.

We are now in the position for a first comparison between $\mathrm{WF}_{\mathrm{G}}$ and $\mathrm{WF}_{\mathrm{SG}}$, which we will continue in Section 2.6.5 (cf. also [121]). From the example of $\delta_{x_{0}}$ it is evident that $\mathrm{WF}_{\mathrm{G}}$ does not share the property of $\mathrm{WF}_{\mathrm{SG}}$ that $\operatorname{pr}_{1}\left(\mathrm{WF}_{\mathrm{SG}}(u)\right)=$ $\operatorname{Css}(u)$ and $\mathrm{WF}_{\mathrm{G}}$ is thus less useful to localize singularities at finite arguments. However, the asymptotic component gives us a better resolution of (quadratic) oscillations, which are condensed in the corner component in the SG case, as can be seen from the example of the chirp $e^{\frac{i}{2} \lambda x^{2}}$ and was visualized in Figure 5 . The example of the chirp also underlines how the SG-wave front set does not share the full invariance under all generators of the symplectic transformations of $\mathrm{WF}_{\mathrm{G}}$. The better resolution of the corner component will also be made evident when we construct a tempered distribution with assigned singularities for the G-wave front in Section 2.6.3. We have already seen in the SG-case that such a construction was highly accessible in the time-frequency plane. This time, we will first introduce how $\mathrm{WF}_{\mathrm{G}}$ can be characterized using the STFT, which will greatly facilitate the construction. 


\subsection{Characterization of $\mathrm{WF}_{\mathrm{G}}$ in the time-frequency plane.}

\subsubsection{Characterization in terms of the STFT.}

As may be expected from the analysis of $\mathrm{WF}_{\mathrm{SG}}, \mathrm{WF}_{\mathrm{G}}$ may be characterized in the time-frequency plane. Due to the simpler structure of $\mathrm{W}_{\mathrm{G}}$ in comparison with $\mathrm{W}_{\mathrm{SG}}$, this takes a simpler form than for $\mathrm{WF}_{\mathrm{SG}}$. In particular we do not need to employ an FBI-transform with two scaling parameters, but may simply use decay properties of the STFT or with reference to one scaling parameter. For Gaussian windows this was established in [82], for general Schwartz windows in [121].

Proposition 2.60 (Characterization of $\mathrm{WF}_{\mathrm{G}}$ via the short-time Fourier transform). Let $u \in \mathscr{S}^{\prime}\left(\mathbb{R}^{d}\right)$ and $\left(x_{0}, \xi_{0}\right) \in \mathrm{W}_{\mathrm{G}}$. Then we have $\left(x_{0}, \xi_{0}\right) \notin \mathrm{WF}_{\mathrm{G}}(u)$ if and only if for one (equivalently all) $\psi \in \mathscr{S}\left(\mathbb{R}^{d}\right) \backslash\{0\}$ there exists an open cone $\Gamma_{0} \subseteq \mathbb{R}^{2 d} \backslash\{0\}$ containing $\left(x_{0}, \xi_{0}\right)$ such that

$$
\sup _{(x, \xi) \in \Gamma_{0}}\langle(x, \xi)\rangle^{N}\left|\mathscr{V}_{\psi} u(x, \xi)\right|<\infty, \quad N \geq 0
$$

Ideas of the proof. Since this result was proved in [121] in great detail, we only list the main ideas of the proof to be able to compare these techniques to those of Section 2.3.1.

Let $a \in \mathrm{G}^{0}\left(\mathbb{R}^{d} \times \mathbb{R}^{d}\right)$ identically 1 for $|(x, \xi)|>R$ and $(x, \xi)$ in a conic neighbourhood of $\left(x_{0}, \xi_{0}\right)$ such that $A u=a^{W}(x, D) u \in \mathscr{S}\left(\mathbb{R}^{d}\right)$. Pick another symbol $b \in \mathrm{G}^{0}\left(\mathbb{R}^{d} \times \mathbb{R}^{d}\right)$ such that $a+b=\mathbb{1}_{\mathbb{R}^{2 d}}$. Then

$$
\mathscr{V}_{\psi_{0}}(u)=\mathscr{V}_{\psi_{0}} \underbrace{A u}_{\in \mathscr{S}}+\mathscr{V}_{\psi_{0}} B u
$$

where the latter term is estimable by using the support properties of $b, \mathrm{~A} .21$ and Lemma A.31.

Conversely, if we have

$$
\sup _{(x, \xi) \in \Gamma}\langle(x, \xi)\rangle^{N}\left|\mathscr{V}_{\psi_{0}} u(x, \xi)\right|<\infty, \quad N \geq 0
$$

then we may find an asymptotic cut-off $\phi^{\left(x_{0}, \xi_{0}\right)}$, equal to 1 for $|(x, \xi)|>R$ and $(x, \xi)$ in a conic neighbourhood $\Gamma^{\prime} \subset \Gamma_{0}$ of $\left(x_{0}, \xi_{0}\right)$ such that $\phi^{\left(x_{0}, \xi_{0}\right)} \mathscr{V}_{\psi_{0}} u \in \mathscr{S}\left(\mathbb{R}^{2 d}\right)$. Then $\mathscr{V}_{\psi_{0}}^{*} \phi^{\left(x_{0}, \xi_{0}\right)} \mathscr{V}_{\psi_{0}}=A_{\phi^{\left(x_{0}, \xi_{0}\right)}}^{\psi_{0}}$ is a localization operator and may, by Proposition A.30, be expressed as a $\Psi D O$ with Weyl symbol $b$ given by (A.19). By direct 
estimates on the convolution (using A.1D) and use of Proposition A.10 it is then possible to prove

- $b \in \mathrm{G}^{0}\left(\mathbb{R}^{d} \times \mathbb{R}^{d}\right)$,

- $b$ is G-elliptic at $\left(x_{0}, \xi_{0}\right)$,

- $b^{W}(x, D) u \in \mathscr{S}\left(\mathbb{R}^{d}\right)$,

which yields the claim for $\psi_{0}$. General windows are then estimated by (A.11).

The characterization of $\mathrm{WF}_{\mathrm{G}}$ in terms of the STFT gives us the opportunity to review Example 2.59, since the STFT for these model examples can be explicitly computed. The graphs of the (absolute values of the) resulting transformed distributions for parameters $\lambda=1$ for the chirp and $\lambda=5$ for the Dirac $\delta$-comb, $x_{0}=0$ and $\xi_{0}=3$ are depicted in Figure 9.28

Dirac $\delta$

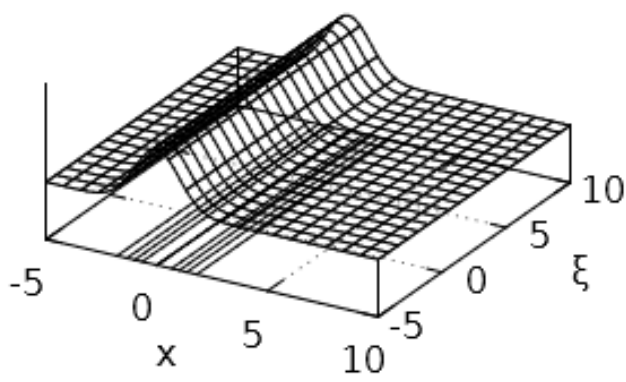

Dirac $\delta$ comb

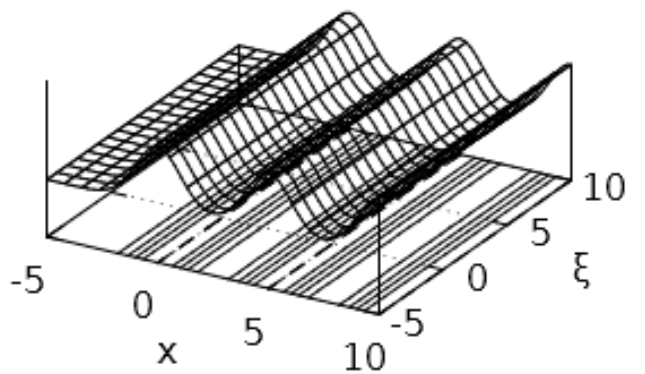

Plane wave

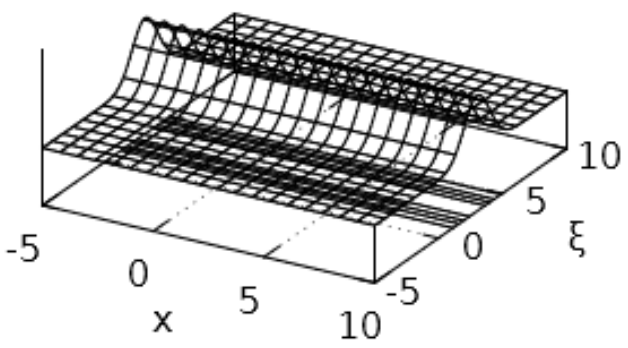

Chirp

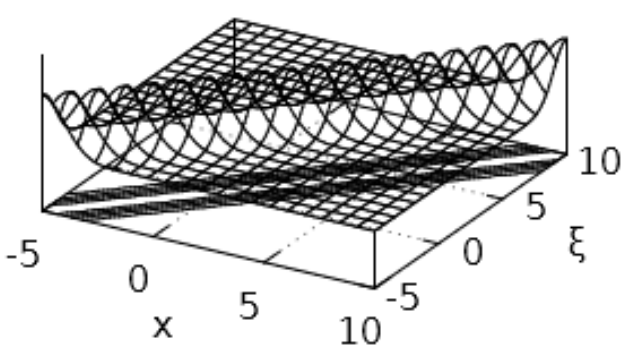

Figure 9. The STFTs of the distributions in Example 2.59

\footnotetext{
${ }^{28}$ The graphs were scaled in amplitude and include level set lines.
} 
With little adaptation, the proof of Proposition 2.60 has the consequence of a characterization of $\mathrm{WF}_{\mathrm{G}}$ in terms of localization operators.

Corollary 2.61. Let $u \in \mathscr{S}^{\prime}\left(\mathbb{R}^{d}\right),\left(x_{0}, \xi_{0}\right) \in \mathrm{W}_{\mathrm{G}}$. Then $\left(x_{0}, \xi_{0}\right) \notin \mathrm{WF}_{\mathrm{G}}(u)$ if and only if there exists $a \in G^{0}\left(\mathbb{R}^{d} \times \mathbb{R}^{d}\right)$ such that

(1) $a$ is an asymptotc cut-off around $\left(x_{0}, \xi_{0}\right)$,

(2) We have $A_{a}^{\psi_{0}} u \in \mathscr{S}\left(\mathbb{R}^{d}\right)$.

Proof. The characterization of $\mathrm{WF}_{\mathrm{G}}$ in terms of the STFT, see the proof of Proposition 2.60, yields the existence of $a$ if $\left(x_{0}, \xi_{0}\right) \notin \mathrm{WF}_{\mathrm{G}}(u)$.

Conversely, if $a$ as in the statement exists, we can use (A.19) to express $A_{a}^{\psi_{0}}$ as a $\Psi D O$ and estimate its Weyl symbol as in [121].

The previous characterization of $\mathrm{WF}_{\mathrm{G}}$ may also be given in terms of an FBI-like transform, or a rescaled STFT with (small) parameter $\hbar .{ }^{29}$ see [130]:

Definition 2.62. Let $u \in \mathscr{S}^{\prime}\left(\mathbb{R}^{d}\right)$. For $\hbar \in(0,1]$ and $x, \xi \in \mathbb{R}^{d}$, the $\hbar$-dependent STFT (short: $\hbar$ STFT) is defined by

$$
\mathscr{V}_{\hbar} u(x, \xi)=(2 \pi)^{-d / 2} \mathcal{D}_{\hbar^{-1}}\left(u, \mathcal{T}_{x} \mathcal{M}_{\xi} \psi_{0}\right)=\mathcal{D}_{\hbar^{-1}}\left(e^{-i \xi x} \mathscr{V}_{\psi_{0}} u\right)
$$

Remark 2.63. We have $\mathscr{V}_{\hbar} u(x, \xi)=\left(\mathscr{F}_{\hbar^{-1}, \hbar^{-1}} u\right)(x, \xi)$, meaning the conic (jointly in $x$ and $\xi$ ) structure of $\mathrm{W}_{\mathrm{G}}$ allows us to replace $\mu$ and $\lambda$ by a single scaling parameter.

Then we have (see [130]) the following characterization of $\mathrm{WF}_{\mathrm{G}}$ in the timefrequency plane:

Proposition 2.64. Let $u \in \mathscr{S}^{\prime}\left(\mathbb{R}^{d}\right)$. Then $\left(x_{0}, \xi_{0}\right) \in \mathrm{W}_{\mathrm{G}}$ is not contained in $\mathrm{WF}_{\mathrm{G}}(u)$ if and only if there exists an open set $U \ni\left(x_{0}, \xi_{0}\right)$ such that

$$
\left\|\left.\mathscr{V}_{\hbar} u\right|_{U}\right\|_{L^{\infty}\left(\mathbb{R}^{2 d}\right)}=\mathcal{O}\left(\hbar^{\infty}\right), \quad \hbar \in(0,1]
$$

Proof. This can be proved by scaling, i.e. taking $U$ as a base for the cone $\Gamma_{0}$ in Proposition 2.60 and using $\left|\mathscr{V}_{\hbar} u(x, \xi)\right|=h^{-d}\left|\mathscr{V}_{\psi_{0}}\left(\hbar^{-1} x, \hbar^{-1} \xi\right)\right|$, see [130].

\footnotetext{
${ }^{29}$ Often $\hbar$ stands for the reduced Planck-constant and the letter $h$ is used to denote the semiclassical parameter. To distinguish it from a test or window function, we instead use $\hbar$ here.
} 
As was shown in [121, the characterization of $\mathrm{WF}_{\mathrm{G}}$ of Proposition 2.60 may be tested on a lattice, using Gabor frames (cf. [68]). Recall that a Gabor frame (or Weyl-Heisenberg frame $) \mathcal{G}(\psi, \alpha, \beta)$ is given by a lattice with parameters $\alpha, \beta>0$, i.e. $\Lambda_{\alpha \beta}=\alpha \mathbb{Z}^{d} \times \beta \mathbb{Z}^{d} \subset \mathbb{R}^{2 d}$ and a (non-zero) window function $\psi \in \mathscr{S}\left(\mathbb{R}^{d}\right)$ such that the collection $\left\{\mathcal{M}_{\xi} \mathcal{T}_{x} \psi\right\}_{(x, \xi) \in \Lambda_{\alpha \beta}}$ is a fram $\varepsilon^{30}$ for $L^{2}\left(\mathbb{R}^{d}\right)$.

Proposition 2.65. Let $u \in \mathscr{S}^{\prime}\left(\mathbb{R}^{d}\right)$. Then $\left(x_{0}, \xi_{0}\right) \in \mathrm{W}_{\mathrm{G}}$ is not contained in $\mathrm{WF}_{\mathrm{G}}(u)$ if and only if for one (equivalently all) Gabor frame $\mathcal{G}(\psi, \alpha, \beta)$ there exists an open cone $\Gamma_{0} \subseteq \mathbb{R}^{2 d} \backslash\{0\}$ containing $\left(x_{0}, \xi_{0}\right)$ such that

$$
\sup _{(x, \xi) \in \Gamma_{0} \cap \Lambda_{\alpha \beta}}\langle(x, \xi)\rangle^{N}\left|\mathscr{V}_{\psi} u(x, \xi)\right|<\infty, \quad N \geq 0 .
$$

This result is important for determination of the G-wave front set by numerical means 31

\subsubsection{G-microlocality of operators.}

The previous results on the characterization of $\mathrm{WF}_{\mathrm{G}}$ in the time-frequency plane may be used to study G-microlocality of various classes of operators. For the simple case of pseudo-differential operators with symbols in $\mathrm{G}^{m}\left(\mathbb{R}^{d} \times \mathbb{R}^{d}\right)$, G-microlocality was stated in Lemma 2.56.

In [121] this was generalized further. We state their result for pseudo-differential operators with symbols in $\mathrm{G}_{0}^{0}\left(\mathbb{R}^{d} \times \mathbb{R}^{d}\right)$, meaning those satisfying

$$
\left|\partial_{x}^{\alpha} \partial_{\xi}^{\beta} a(x, \xi)\right| \lesssim C \quad \forall \alpha, \beta \in \mathbb{N}_{0}^{d} .
$$

Proposition 2.66. Let $a \in \mathrm{G}_{0}^{0}\left(\mathbb{R}^{d} \times \mathbb{R}^{d}\right), u \in \mathscr{S}^{\prime}\left(\mathbb{R}^{d}\right)$. Then $\mathrm{WF}_{\mathrm{G}}\left(a^{W}(x, D) u\right) \subset$ $\mathrm{WF}_{\mathrm{G}}(u)$, i.e. $a^{W}(x, D): \mathscr{S}^{\prime}\left(\mathbb{R}^{d}\right) \rightarrow \mathscr{S}^{\prime}\left(\mathbb{R}^{d}\right)$ is G-microlocal.

Using the Weyl-Wick connection, i.e. Proposition A.30, we can extend these results to localization operators.

Proposition 2.67. Let $u \in \mathscr{S}^{\prime}\left(\mathbb{R}^{d}\right)$ and

$$
a \in \mathrm{G}^{m}\left(\mathbb{R}^{d} \times \mathbb{R}^{d}\right) \text { or } a \in \mathrm{G}_{0}^{0}\left(\mathbb{R}^{d} \times \mathbb{R}^{d}\right) .
$$

${ }^{30}$ Recall: a sequence of $\left.v_{\alpha} \in \mathcal{H},\left(\mathcal{H},(\cdot, \cdot)_{\mathcal{H}}\right)\right)$ a separable Hilbert space, is called a frame, if $\exists B \geq A>0$ s.t. for all $w \in \mathcal{H}$ we have

$$
A\|w\|_{\mathcal{H}} \leq \sum_{\alpha}\left|\left(w, v_{\alpha}\right)_{\mathcal{H}}\right|^{2} \leq B\|w\|_{\mathcal{H}}
$$

${ }^{31}$ It further provides the original reason why this notion of singularities was called "Gabor wave front set", i.e. $\mathrm{WF}_{\mathrm{G}}$, in [121]. 
Then $A_{a}^{\psi_{0}}: \mathscr{S}^{\prime}\left(\mathbb{R}^{d}\right) \rightarrow \mathscr{S}^{\prime}\left(\mathbb{R}^{d}\right)$ is G-microlocal.

Proof. Use Peetre's inequality (A.1) (as in the proof of Proposition 2.60, see [121]) to show that $a$ either symbol space implies that A.19) yields an amplitude in the same symbol space. Then use the microlocality of such $\Psi$ DOs, i.e. Lemma 2.56 or Proposition 2.66 .

This further underlines that the G-wave front set is a notion of singularity well-adapted to problems of time-frequency analysis, in particular localization operators. We refer the reader to [30, 31] for a study of propagation of singularities under (almost) metaplectic operators.

\subsubsection{Existence of distributions with assigned singularities.}

In this section, we repeat ${ }^{32}$ the construction first obtained in collaboration with Wahlberg in [130] which yields, for any closed conic set $\Gamma_{0} \subseteq \mathbb{R}^{2 d} \backslash\{0\}$, a distribution $u \in \mathscr{S}^{\prime}\left(\mathbb{R}^{d}\right)$ with $\mathrm{WF}_{\mathrm{G}}(u)=\Gamma_{0}$. It is based on the construction used in the SG-setting to prove Corollary 2.17.

Again, $u$ is constructed in terms of modulated and translated Gaussian functions, and thus we can calculate its STFT in terms of elementary functions and simply read off its decay properties.

Recall the shifted Gaussians of Definition 2.14. Using (A.9) and performing a simple Gaussian integration, we may compute the STFT of $\psi_{k}\left(\cdot, x_{0}, \xi_{0}\right)$ for $\left(x_{0}, \xi_{0}\right) \in \mathrm{W}_{\mathrm{G}}$ :

$$
\begin{aligned}
\mathscr{V}_{\psi_{0}} & \left(\psi_{k}\left(\cdot ; x_{0}, \xi_{0}\right)\right)(x, \xi)=(2 \pi)^{-\frac{d}{2}} e^{-\frac{i}{2} k^{4} x_{0} \xi_{0}}\left(\psi_{0}, \mathcal{M}_{\xi-k^{2} \xi_{0}} \mathcal{T}_{x-k^{2} x_{0}} \psi_{0}\right) \\
& =\left(\frac{\pi}{2}\right)^{\frac{d}{2}} e^{-\frac{i}{2} k^{4} x_{0} \xi_{0}} e^{-\frac{i}{2}\left(x-k^{2} x_{0}\right)\left(\xi-k^{2} \xi_{0}\right)} \exp \left(-\frac{1}{4}\left(\left|x-k^{2} x_{0}\right|^{2}+\left|\xi-k^{2} \xi_{0}\right|^{2}\right)\right) .
\end{aligned}
$$

In particular we have

$$
\left|\mathscr{V}_{\psi_{0}}\left(\psi_{k}\left(\cdot ; x_{0}, \xi_{0}\right)\right)(x, \xi)\right|=\left(\frac{\pi}{2}\right)^{\frac{d}{2}} \exp \left(-\frac{1}{4}\left(\left|x-k^{2} x_{0}\right|^{2}+\left|\xi-k^{2} \xi_{0}\right|^{2}\right)\right)
$$

For any $f \in \mathscr{S}\left(\mathbb{R}^{d}\right)$ we further have, by direct comparison of the definition of the STFT and the $\psi_{k}$ :

$$
\left(\psi_{k}, f\right)=2^{d / 2} e^{-\frac{i}{2} k^{4} x_{0} \xi_{0}} \overline{\mathscr{V}_{\psi_{0}} f\left(k^{2} x_{0}, k^{2} \xi_{0}\right)} .
$$

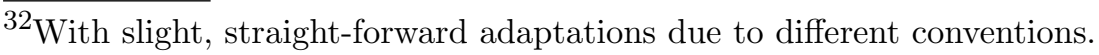


Since $\mathscr{V}_{\psi_{0}} f \in \mathscr{S}\left(\mathbb{R}^{2 d}\right)$, by Lemma A.9, and $\left(x_{0}, \xi_{0}\right) \neq 0$, the series

$$
v\left(\cdot ; x_{0}, \xi_{0}\right)=\sum_{k=1}^{\infty} \psi_{k}\left(\cdot ; x_{0}, \xi_{0}\right)
$$

converges in $\mathscr{S}^{\prime}\left(\mathbb{R}^{d}\right)$, which gives $v\left(\cdot ; x_{0}, \xi_{0}\right) \in \mathscr{S}^{\prime}\left(\mathbb{R}^{d}\right){ }^{33}$

We may summarize the upcoming construction in a few lines: several copies of $v\left(\cdot, x_{j}, \xi_{j}\right)$ are summed up to construct a tempered distribution whose STFT is rapidly decaying everywhere, except in the directions given by the closure of $\left\{x_{j}, \xi_{j}\right\} \subset \mathbb{S}^{2 d-1}$, i.e. $\Gamma_{0}$. That this procedure yields the claimed result can be understood by considering Figure 10 , where the graph of the STFT of $(v ; 1,1)$ is depicted 34

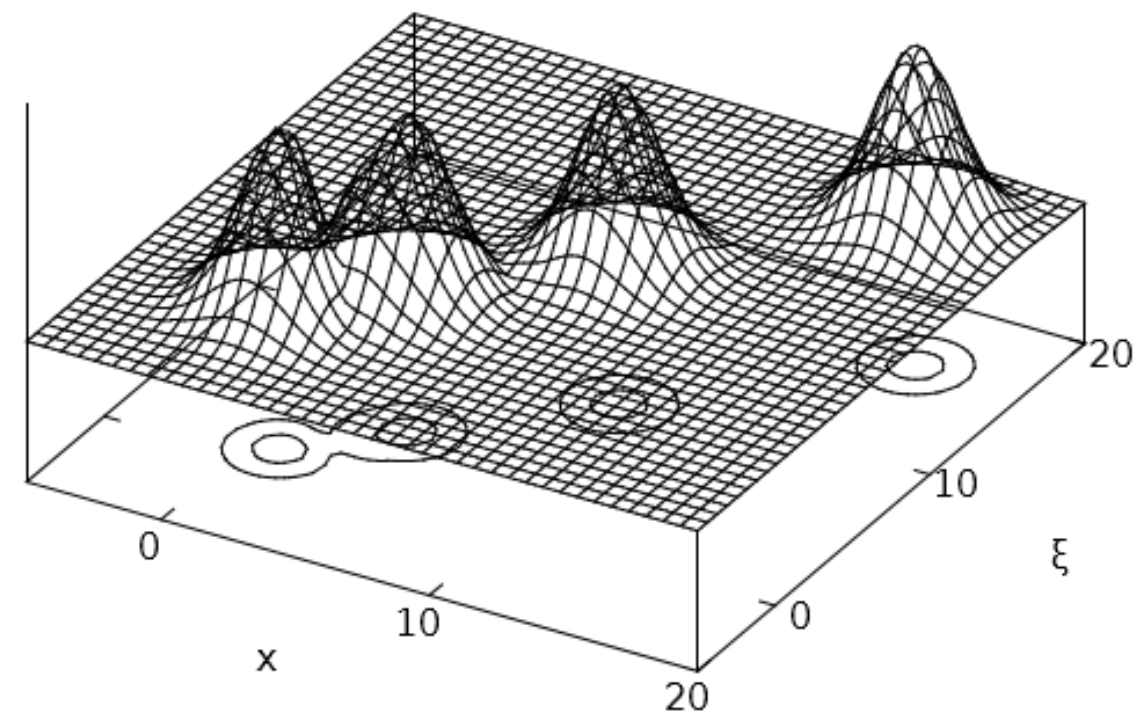

FiguRE 10. The graph of $\left|\mathscr{V}_{\psi_{0}} v(\cdot ; 1,1)\right|$ (up to $k=4$ )

Theorem 2.68. For any closed set $\Gamma \subseteq \mathrm{W}_{\mathrm{G}}$ there exists $u \in \mathscr{S}^{\prime}\left(\mathbb{R}^{d}\right)$ such that $\mathrm{WF}_{\mathrm{G}}(u)=\Gamma$.

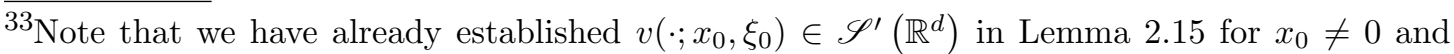
$\xi_{0} \neq 0$. Therein, we studied the cone singular support of $v$. Here we lay specific weight on the explicit form of its action in terms of the STFT, i.e. (2.25), and also address the cases where either $x_{0}$ or $\xi_{0}$ may vanish.

${ }^{34}$ Figure 10 may be seen as a non-schematic version of Figure 7 the STFT allows us to obtain a visualization of $v(x ; 1,1)$ in the time-frequency plane.
} 
Proof. Take a dense subset of distinct vectors $\left\{w_{j}\right\}_{j \in \mathbb{N}_{0}} \subseteq \Gamma_{0} \cap \mathbb{S}^{2 d-1}$. Then define, by means of 2.25 ,

$$
u:=\sum_{j=0}^{\infty} 2^{-j} v\left(\cdot ; w_{j}\right), \quad x \in \mathbb{R}^{d} .
$$

We have $u \in \mathscr{S}^{\prime}\left(\mathbb{R}^{d}\right)$, since for all $f \in \mathscr{S}\left(\mathbb{R}^{d}\right)$, by 2.25$)$ and 2.24$)$, it holds that

$$
\left|\left(v\left(\cdot ; w_{j}\right), f\right)\right| \leq \sum_{k=1}^{\infty}\left|\left(\psi_{k}\left(\cdot ; w_{j}\right), f\right)\right|=2^{d / 2} \sum_{k=1}^{\infty}\left|\left(\mathscr{V}_{\psi_{0}} f\right)\left(k^{2} w_{j}\right)\right| .
$$

Since $\mathscr{V}_{\psi_{0}} f \in \mathscr{S}\left(\mathbb{R}^{2 d}\right)$ and $\left|w_{j}\right|=1$ it follows that $\left|\left(v\left(\cdot ; w_{j}\right), f\right)\right|$ is bounded by a constant, uniformly over $j \in \mathbb{N}_{0}$. This in turn shows that the sum over $j$ in $(2.26)$ converges in $\mathscr{S}^{\prime}\left(\mathbb{R}^{d}\right)$.

We now show that $\mathrm{WF}_{\mathrm{G}} \subset \Gamma_{0}$. For that consider $z_{0} \in \mathbb{S}^{2 d-1}$ such that $z_{0} \notin \Gamma_{0}$. By Lemma 1.4 there exists an open conic set $\Gamma \subseteq \mathbb{R}^{2 d} \backslash\{0\}$ and $\epsilon>0$ such that $z_{0} \in \Gamma$, and for any $z \in \Gamma, k \geq 1, w \in \Gamma_{0}$ we have

$$
\left|z-k^{2} w\right| \geq \epsilon\left(|z|+k^{2}\right) .
$$

Using (2.23), this gives for $z \in \Gamma_{0}$ and $N \geq 0$ arbitrary

$$
\begin{aligned}
\langle z\rangle^{N}\left|\mathscr{V}_{\psi_{0}} u(z)\right| & \leq \sum_{j=0}^{\infty} 2^{-j} \sum_{k=1}^{\infty}\langle z\rangle^{N}\left|\mathscr{V}_{\psi_{0}} \psi_{k}\left(\cdot ; w_{j}\right)(z)\right| \\
& \lesssim \sum_{k=1}^{\infty}\langle z\rangle^{N} \exp \left(-\frac{\epsilon^{2}}{4}\left(|z|^{2}+k^{4}\right)\right)<\infty
\end{aligned}
$$

Thus $z_{0} \notin \mathrm{WF}_{\mathrm{G}}(u)$, which proves $\mathrm{WF}_{\mathrm{G}}(u) \subseteq \Gamma_{0}$ since $z_{0} \notin \Gamma_{0}$ was arbitrary.

On the other hand we show that $w_{m}$, for $m \in \mathbb{N}_{0}$ fixed is in $\mathrm{WF}_{\mathrm{G}}(u)$. Then, since $\left\{w_{j}\right\}_{j \in \mathbb{N}_{0}}$ is dense in $\Gamma_{0} \cap \mathbb{S}^{2 d-1}$, and since $\mathrm{WF}_{\mathrm{G}}(u) \subset \mathrm{W}_{\mathrm{G}}$ is a closed conic set, we have $\Gamma_{0} \subset \mathrm{WF}_{\mathrm{G}}(u)$. In order to show $w_{m} \in \mathrm{WF}_{\mathrm{G}}(u)$, we prove a lower bound on

$$
\exists \epsilon>0 \text { such that } \limsup _{\lambda \rightarrow \infty}\left|\mathscr{V}_{\psi_{0}} u\left(\lambda w_{m}\right)\right|>\epsilon .
$$

by showing that at a "peak" at $n^{2} w_{m}$ (consider Figure 10), all summands except one - originating from the Gaussian centred around it - become negligible for suitably large arguments. We dedicate the rest of the proof to detailed estimates

\footnotetext{
$\overline{{ }^{35} \text { I.e. the } w_{j}}$ are of the form $\left(x_{j}, \xi_{j}\right)$. If $\Gamma_{0} \cap \mathbb{S}^{2 d-1}$ happens to be finite, simply take all of its elements.
} 
to obtain the lower bound 2.27).

Let $m \in \mathbb{N}_{0}$ fixed and $n \geq 1$ be an integer to be specified later on. Formula 2.23) gives

$$
\left|\mathscr{V}_{\psi_{0}}\left(v\left(\cdot ; w_{m}\right)\right)\left(n^{2} w_{m}\right)\right| \geq\left(\frac{\pi}{2}\right)^{d / 2}-\sum_{k=1, k \neq n}^{\infty}\left|\mathscr{V}_{\psi_{0}}\left(\psi_{k}\left(\cdot ; w_{m}\right)\right)\left(n^{2} w_{m}\right)\right|
$$

The same formula yields

$$
\begin{aligned}
\left|\mathscr{V}_{\psi_{0}}\left(\psi_{k}\left(\cdot ; w_{j}\right)\right)\left(n^{2} w_{m}\right)\right| & =\left(\frac{\pi}{2}\right)^{d / 2} \exp \left(-\frac{1}{4}\left|n^{2} w_{m}-k^{2} w_{j}\right|^{2}\right) \\
& \leq\left(\frac{\pi}{2}\right)^{d / 2} \exp \left(-\frac{1}{4}(n+k)^{2}(n-k)^{2}\right), \quad j \in \mathbb{N}_{0}
\end{aligned}
$$

This gives

$$
\sum_{k \neq n}\left|\mathscr{V}_{\psi_{0}}\left(\psi_{k}\left(\cdot ; w_{j}\right)\right)\left(n^{2} w_{m}\right)\right| \longrightarrow 0, \quad n \rightarrow+\infty
$$

independently of $j \in \mathbb{N}_{0}$. Inserting $j=m$, we obtain from 2.28 the lower bound

$$
\left|\mathscr{V}_{\psi_{0}}\left(v\left(\cdot ; w_{m}\right)\right)\left(n^{2} w_{m}\right)\right| \geq \frac{1}{2}\left(\frac{\pi}{2}\right)^{d / 2}, \quad n \geq N,
$$

for some integer $N \geq 1$, which yields for $n \geq N$

$$
\begin{aligned}
\left|\left(\mathscr{V}_{\psi_{0}} u\right)\left(n^{2} w_{m}\right)\right| & =\left(\frac{\pi}{2}\right)^{d / 2}\left|\sum_{j=0}^{\infty} 2^{-j} \mathscr{V}_{\psi_{0}}\left(v\left(\cdot ; w_{j}\right)\right)\left(n^{2} w_{m}\right)\right| \\
& \geq\left(\frac{\pi}{2}\right)^{d / 2}\left(2^{-m-1}-\sum_{j=0, j \neq m}^{\infty} 2^{-j}\left|\mathscr{V}_{\psi_{0}}\left(v\left(\cdot ; w_{j}\right)\right)\left(n^{2} w_{m}\right)\right|\right) .
\end{aligned}
$$

We are now able to estimate the remainder. From (2.25), 2.29) and 2.30 we obtain

$$
\left|\mathscr{V}_{\psi_{0}}\left(v\left(\cdot ; w_{j}\right)\right)\left(n^{2} w_{m}\right)\right| \leq 2\left(\frac{\pi}{2}\right)^{d / 2}, \quad j \in \mathbb{N}_{0}, \quad n \geq N
$$

after possibly increasing $N$. Since this bound is uniform with respect to $j \in \mathbb{N}_{0}$, it implies that the existence of an integer $M \geq m+1$ such that

$$
\sum_{j=M}^{\infty} 2^{-j}\left|\mathscr{V}_{\psi_{0}}\left(v\left(\cdot ; w_{j}\right)\right)\left(n^{2} w_{m}\right)\right| \leq 2^{-m-3}\left(\frac{\pi}{2}\right)^{d / 2}, \quad n \geq N
$$


For $0 \leq j \leq M-1$ and $j \neq m$ we have $\left|w_{m}-w_{j}\right| \geq \delta$ for some $\delta>0$. Combining this fact with the previous (in-)equalities (2.23), 2.25) and 2.30), yields

$$
\begin{aligned}
& \left|\mathscr{V}_{\psi_{0}}\left(v\left(\cdot ; w_{j}\right)\right)\left(n^{2} w_{m}\right)\right| \\
& \leq\left|\mathscr{V}_{\psi_{0}}\left(\psi_{n}\left(\cdot ; w_{j}\right)\right)\left(n^{2} w_{m}\right)\right|+\sum_{k \neq n}\left|\mathscr{V}_{\psi_{0}}\left(\psi_{k}\left(\cdot ; w_{j}\right)\right)\left(n^{2} w_{m}\right)\right| \\
& \leq\left(\frac{\pi}{2}\right)^{d / 2}\left(\exp \left(-n^{4} \delta^{2} / 4\right)+2^{-m-5}\right) \leq 2^{-m-4}\left(\frac{\pi}{2}\right)^{d / 2}, \quad 0 \leq j \leq M-1, n \geq N,
\end{aligned}
$$

again after possibly increasing $N$. Summing up, we thus obtain

$$
\sum_{j=0, j \neq m}^{M-1} 2^{-j}\left|\mathscr{V}_{\psi_{0}}\left(v\left(\cdot ; w_{j}\right)\right)\left(n^{2} w_{m}\right)\right| \leq 2^{-m-3}\left(\frac{\pi}{2}\right)^{d / 2}, \quad n \geq N
$$

Combining the two estimates $(2.32)$ and $(2.33)$ for $j \neq m$ and inserting them into (2.31) we finally obtain the lower bound

$$
\left|\left(\mathscr{V}_{\psi_{0}} u\right)\left(n^{2} w_{m}\right)\right| \geq 2^{-m-2}\left(\frac{\pi}{2}\right)^{d / 2}
$$

for $n \geq N$. Consequently, $\mathscr{V}_{\psi_{0}} u$ does not decay rapidly in any conic neighbourhood of $w_{m}$, which concludes the proof.

\subsubsection{A semi-classical description.}

This section is devoted to another characterization of $\mathrm{WF}_{\mathrm{G}}$ in the time-frequency plane, via semiclassical methods. Consider first the characterization of $\mathrm{WF}_{\mathrm{G}}$ by means of the STFT and by the G-pseudo-differential calculus. Both approaches can be thought of as localizing $u$ in the time-frequency plane in a fixed cone and testing for rapid decay.

We have already seen in Definition 2.62 how equivalently a small semiclassical dilation parameter $\hbar$ may be introduced in both phase space variables simultaneously to characterize $\mathrm{WF}_{\mathrm{G}}$ by localizing in a finite neighbourhood of the time-frequency plane by scaling behaviour. The same picture on the side of pseudo-differential operators is achieved by the notion of homogeneous wave front set of Nakamura [111].

Definition 2.69. Let $u \in \mathscr{S}^{\prime}\left(\mathbb{R}^{d}\right)$. A point $\left(x_{0}, \xi_{0}\right) \in \mathrm{W}_{\mathrm{G}}$ is not in the homogeneous wave front set $\operatorname{HWF}(u)$ if there exists $a \in \mathscr{C}_{c}^{\infty}\left(\mathbb{R}^{2 d}\right)$ with $a\left(x_{0}, \xi_{0}\right)=1$ such 
that

$$
\left\|a_{\hbar}^{W}(x, D) u\right\|_{L^{2}}=\left\|a^{W}(\hbar x, \hbar D) u\right\|_{L^{2}}=\mathcal{O}\left(\hbar^{\infty}\right) \text { for } \hbar \in(0,1] .
$$

where $a^{W}(\hbar x, \hbar D)$ is the operator $\mathscr{S}^{\prime}\left(\mathbb{R}^{d}\right) \rightarrow \mathscr{S}\left(\mathbb{R}^{d}\right)$ that is obtained as the Weyl quantization of $a(\hbar x, \hbar \xi)$.

This notion of wave front set was used to study propagation of singularities for Schrödinger equations, see [111]. "Analogons" of it for various functional settings were already defined in terms of the STFT, see [100, 101, 109]. Here we focus on the smooth case. In this case, there is a connection with the quadratic scattering wave front set of [153, 154]. This notion is a modification ("blow up in the corner") of the scattering wave front set and may be defined in the qsc-calculus, which may be obtained by changing the boundary defining function of the compactification of $\mathbb{R}^{d}$. The wave front set then encodes the usual one and quadratic singularities at infinity.

The following theorem was established in [85, 86]:

Theorem 2.70. Let $\Psi: \mathbb{R}^{d} \backslash\{0\} \rightarrow \mathrm{GL}(d, \mathbb{R})$ defined via

$$
\Psi(x)=\left(\delta_{j k}+\frac{x^{j} x^{k}}{|x|^{2}}\right)_{j, k} .
$$

Then we have, for $u \in \mathscr{S}^{\prime}\left(\mathbb{R}^{d}\right)$, the equality

$$
\{(x, \Psi(x) \xi) \mid(x, \xi) \in \operatorname{HWF}(u), x \neq 0\}=\mathbb{R}_{+} \cdot\left({ }^{\mathrm{qsc}} \mathrm{WF}(u) \cap\left(\mathbb{S}^{d-1} \times \mathbb{R}^{d}\right)\right)
$$

Remark 2.71. Despite this connection, we will only make use of $\mathrm{WF}_{\mathrm{G}}$, since the $\{0\} \times\left(\mathbb{R}^{d} \backslash\{0\}\right)$-component of $\mathrm{WF}_{\mathrm{G}}$ carries essential information for our purposes, see Proposition 2.86.

Before discussing how $\mathrm{HWF}$ and $\mathrm{WF}_{\mathrm{G}}$ relate, we first review the classical setting. In semi-classical analysis, one introduces the so-called frequency set (consider [64, 99, 157]) in terms of its complement in $T^{*} \mathbb{R}^{d}$ as follows:

Definition 2.72. Let $\left(u_{\hbar}\right)_{\hbar \in(0,1]}$ a bounded family of elements in $\mathscr{S}^{\prime}\left(\mathbb{R}^{d}\right)$. Then $\left(x_{0}, \xi_{0}\right) \in T^{*} \mathbb{R}^{d}$ is not in $\operatorname{FS}\left(u_{\hbar}\right)$ if there exists $a \in \mathscr{C}_{c}^{\infty}\left(\mathbb{R}^{d}\right)$ with $a\left(x_{0}, \xi_{0}\right)=1$ such that

$$
\left\|a^{W}(x, \hbar D) u_{\hbar}\right\|_{2}=\mathcal{O}\left(\hbar^{\infty}\right) .
$$


When $u$ does not depend on $\hbar$, this essentially coincides with the classical wave front set:

Proposition 2.73. Let $u \in \mathscr{S}^{\prime}\left(\mathbb{R}^{d}\right)$. Then $\operatorname{FS}(u)=\mathrm{WF}_{\mathrm{cl}}(u) \sqcup(\operatorname{supp}(u) \times\{0\})$. Thus FS provides a description of $\mathrm{WF}_{\mathrm{cl}}$ by testing in a small (time-frequency) neighbourhood and scaling by the small parameter $\hbar$. This result is the initial motivation for the introduction of HWF, see [111].

Additionally, we have the description of $\mathrm{WF}_{\mathrm{cl}}$ by the FBI-transform $\mathscr{F}_{\lambda}$ in Proposition 2.18. Setting $\hbar=\lambda^{-1}$, we obtain a description of $\mathrm{WF}_{\mathrm{cl}}$ similar to the one of $\mathrm{WF}_{\mathrm{G}}$ of Proposition 2.64 (cf. [157, Thm. 13.14]).

It is therefore reasonable to assume that $\mathrm{HWF}$ and $\mathrm{WF}_{\mathrm{G}}$ should be related in the same way as $\mathrm{FS}$ and $\mathrm{WF}_{\mathrm{cl}}$ are. Indeed, we have the following equivalence, which was proven in [130]:

Theorem 2.74. Let $u \in \mathscr{S}^{\prime}\left(\mathbb{R}^{d}\right)$. Then $\mathrm{WF}_{\mathrm{G}}(u)=\operatorname{HWF}(u)$.

In the following, we repeat the proof for Theorem 2.74 obtained in collaboration with Wahlberg in [130]. First we need to state certain results about operators of the form $\mathrm{Op}_{t} a(\hbar x, \hbar D)$. While in the appendix of [130] a calculus for such operators is worked out in detail, here we just state the results needed in order to prove Theorem 2.74. They allow for us to choose the operator in the definition of HWF to have additional properties and to change quantization.

Lemma 2.75. Let $u \in \mathscr{S}^{\prime}\left(\mathbb{R}^{d}\right)$ and $a \in \mathscr{C}_{c}^{\infty}\left(\mathbb{R}^{2 d}\right),\left(x_{0}, \xi_{0}\right) \in\left(\mathbb{R}^{2 d} \backslash\{0\}\right)$, $a\left(x_{0}, \xi_{0}\right)=1$ and

$$
\left\|a^{W}(\hbar x, \hbar D) u\right\|_{L^{2}}=\mathcal{O}\left(\hbar^{\infty}\right), \quad \hbar \in(0,1]
$$

Then there exists a bounded neighbourhood $U$ of $\left(x_{0}, \xi_{0}\right)$, such that for any $b \in$ $\mathscr{C}_{c}^{\infty}\left(\mathbb{R}^{2 d}\right)$ with $\operatorname{supp}(b) \subseteq U$ we have

$$
\left\|b^{W}(\hbar x, \hbar D) u\right\|_{L^{2}}=\mathcal{O}\left(\hbar^{\infty}\right), \quad \hbar \in(0,1] .
$$

Furthermore, there exists $\tilde{a} \in \mathscr{C}_{c}^{\infty}\left(\mathbb{R}^{2 d}\right)$ supported in a neighbourhood of $z_{0}$ and equal to one in a (smaller) neighbourhood of $\left(x_{0}, \xi_{0}\right)$, such that

$$
\|\tilde{a}(\hbar x, \hbar D) u\|_{L^{2}}=\mathcal{O}\left(\hbar^{\infty}\right), \quad \hbar \in(0,1] .
$$

Next, we need two lemmas which can be used to estimate the action of an operator of the form $\mathrm{Op}_{t} a_{\hbar}(x, D)$ on modulated and translated Gaussians, i.e. on $\mathscr{V}_{\hbar} u$ 
in some neighbourhood when $a$ satisfies certain support properties. Both Lemmas can be obtained by integral regularization using stationary phase methods, i.e. repeated partial integration, consider [130, Sect. 3].

Lemma 2.76. Suppose $a \in \mathrm{G}^{0}\left(\mathbb{R}^{d} \times \mathbb{R}^{d}\right)$ vanishes in a neighbourhood of $\left(x_{0}, \xi_{0}\right) \in$ $\mathbb{R}^{2 d}$. Then there exists a (smaller) neighbourhood $V$ of $\left(x_{0}, \xi_{0}\right)$ such that for any $N, M \in \mathbb{N}_{0}$,

$$
\rho_{N}^{\mathscr{S}}\left(a^{R}\left(\hbar y, \hbar D_{y}\right)\left(\mathcal{T}_{x / \hbar} \mathcal{M}_{\xi / \hbar} \psi_{0}\right)\right) \leq C_{N, M} \hbar^{M}, \quad \hbar \in(0,1], \quad(x, \xi) \in V .
$$

Lemma 2.77. Let $\left(x_{0}, \xi_{0}\right) \in \mathbb{R}^{2 d}$ and $\epsilon>0$. Suppose $a \in \mathscr{C}_{c}^{\infty}\left(\mathbb{R}^{2 d}\right)$ with

$$
\operatorname{supp}(a) \subseteq \mathbb{B}_{\epsilon / 4}^{d}\left(x_{0}, \xi_{0}\right)=\left\{(x, \xi) \in \mathbb{R}^{d}||(x, \xi)-\left(x_{0}, \xi_{0}\right) \mid \leq \frac{\epsilon}{4}\right\} .
$$

For all $(x, \xi) \in \mathbb{R}^{2 d}$ such that

$$
\left|x-x_{0}\right|^{2}+\left|\xi-\xi_{0}\right|^{2} \geq \epsilon^{2}>0,
$$

there exists, for all $N \in \mathbb{N}_{0}$, a constant $C_{N}>0$ such that

$$
\left\|a^{W}\left(\hbar y, \hbar D_{y}\right)\left(\mathcal{T}_{x / \hbar} \mathcal{M}_{\xi / \hbar} \psi_{0}\right)\right\|_{L^{2}}^{2} \leq C_{N} \hbar^{2 N}\langle(x, \xi)\rangle^{-N}, \quad \hbar \in(0,1] .
$$

Proof of Theorem 2.74. $\mathrm{WF}_{\mathrm{G}}(u) \subset \operatorname{HWF}(u)$ :

Suppose $\left(x_{0}, \xi_{0}\right) \notin \operatorname{HWF}(u)$ and $\left(x_{0}, \xi_{0}\right) \neq(0,0)$. Then, by the Definition 2.69 of $\operatorname{HWF}(u)$ and Lemma 2.75, there exists $a \in \mathscr{C}_{c}^{\infty}\left(\mathbb{R}^{2 d}\right)$ with $a=1$ in a neighbourhood of $\left(x_{0}, \xi_{0}\right)$ such that

$$
\left\|a^{W}\left(\hbar y, \hbar D_{y}\right) u\right\|_{L^{2}\left(\mathbb{R}^{d}\right)} \lesssim \hbar^{N}, \quad \hbar \in(0,1], \quad N \geq 0 .
$$

By Lemma 2.75 we may instead take a different symbol $b$ and the left quantization, thus assuming

$$
\left\|b\left(\hbar y, \hbar D_{y}\right) u\right\|_{L^{2}\left(\mathbb{R}^{d}\right)} \lesssim \hbar^{N}, \quad \hbar \in(0,1], \quad N \geq 0 .
$$

Splitting $u=b\left(\hbar y, \hbar D_{y}\right) u+(1-b)\left(\hbar y, \hbar D_{y}\right) u$ we obtain, by use of Definition 2.62 and the Cauchy-Schwarz inequality, for any $(x, \xi) \in \mathbb{R}^{2 d}$

$$
\begin{aligned}
\left|\mathscr{V}_{\hbar}\left(b\left(\hbar y, \hbar D_{y}\right) u\right)(x, \xi)\right| & \leq(2 \pi)^{-d / 2} \hbar^{-d}\left\|b\left(\hbar y, \hbar D_{y}\right) u\right\|_{L^{2}\left(\mathbb{R}^{d}\right)}\left\|\psi_{0}\right\|_{L^{2}} \\
& \lesssim \hbar^{N-d}, \quad \hbar \in(0,1], \quad N \geq 0 .
\end{aligned}
$$


From Lemma A.19 i.e. $(b(x, D) u, g)=\left(u, \bar{b}^{R}(x, D) g\right)$ for $g \in \mathscr{S}\left(\mathbb{R}^{d}\right)$, and Lemma 2.76, we obtain for some $m, k \in \mathbb{N}_{0}$

$$
\begin{aligned}
\mid \mathscr{V}_{\hbar}((1-b) & \left.\left(\hbar y, \hbar D_{y}\right) u\right)(x, \xi) \mid \\
& =(2 \pi)^{-d / 2} \hbar^{-d}\left|\left((1-b)\left(\hbar y, \hbar D_{y}\right) u, \mathcal{T}_{x / \hbar} \mathcal{M}_{\xi / \hbar} \psi_{0}\right)\right| \\
& =(2 \pi)^{-d / 2} \hbar^{-d}\left|\left(u,(1-\bar{b})^{R}\left(\hbar y, \hbar D_{y}\right)\left(\mathcal{T}_{x / \hbar} \mathcal{M}_{\xi / \hbar} \psi_{0}\right)\right)\right| \\
& \lesssim \hbar^{-d} \rho_{N}\left((1-\bar{b})^{R}\left(\hbar y, \hbar D_{y}\right)\left(\mathcal{T}_{x / \hbar} \mathcal{M}_{\xi / \hbar} \psi_{0}\right)\right) \\
& \lesssim \hbar^{M-d}, \quad \hbar \in(0,1], \quad M \geq 0,
\end{aligned}
$$

for $(x, \xi)$ in a sufficiently small neighbourhood of $\left(x_{0}, \xi_{0}\right)$. Combining with 2.37) we may conclude for some small $\delta>0$

$$
\sup _{(x, \xi) \in \mathbb{B}_{\delta}^{d}\left(x_{0}, \xi_{0}\right)}\left|\mathscr{V}_{\hbar} u(x, \xi)\right| \lesssim \hbar^{N}, \quad \hbar \in(0,1], \quad N \geq 0,
$$

and by Proposition 2.64 it follows that $\left(x_{0}, \xi_{0}\right) \notin \mathrm{WF}_{\mathrm{G}}(u)$, which proves $\mathrm{WF}_{\mathrm{G}}(u) \subseteq$ $\operatorname{HWF}(u)$.

$\operatorname{HWF}(u) \subset \mathrm{WF}_{\mathrm{G}}(u):$

Suppose that $0 \neq\left(x_{0}, \xi_{0}\right) \notin \mathrm{WF}_{\mathrm{G}}(u)$. By means of the Moyal identity (A.8) and Lemma A.19 we obtain for $a \in \mathscr{C}_{c}^{\infty}\left(\mathbb{R}^{2 d}\right)$

$$
\begin{aligned}
& \left\|a^{W}\left(\hbar y, \hbar D_{y}\right) u\right\|_{L^{2}} \\
& \quad=\sup _{g \in \mathscr{S}\left(\mathbb{R}^{d}\right),\|g\|=1}\left|\left(a^{W}\left(\hbar y, \hbar D_{y}\right) u, g\right)\right| \\
& \quad=\sup _{g \in \mathscr{S}\left(\mathbb{R}^{d}\right),\|g\|=1}\left|\left(u, \bar{a}^{W}\left(\hbar y, \hbar D_{y}\right) g\right)\right| \\
& \quad \lesssim \sup _{g \in \mathscr{S}\left(\mathbb{R}^{d}\right),\|g\|=1} \hbar^{-d} \int_{\mathbb{R}^{2 d}}\left|\mathscr{V}_{\hbar} u(x, \xi)\left(a_{\hbar}^{W}\left(y, D_{y}\right)\left(\mathcal{T}_{x / \hbar} \mathcal{M}_{\xi / \hbar} \psi_{0}\right), g\right)_{L^{2}}\right| d x d \xi \\
& \quad \lesssim \hbar^{-d} \int_{\mathbb{R}^{2 d}}\left|\mathscr{V}_{\hbar} u(x, \xi)\right|\left\|a_{\hbar}^{W}\left(y, D_{y}\right)\left(\mathcal{T}_{x / \hbar} \mathcal{M}_{\xi / \hbar} \psi_{0}\right)\right\|_{L^{2}} d x d \xi .
\end{aligned}
$$

By Proposition 2.64 there exists a bounded open neighbourhood $U \ni\left(x_{0}, \xi_{0}\right)$ such that

$$
\sup _{(x, \xi) \in U}\left|\mathscr{V}_{\hbar} u(x, \xi)\right| \lesssim \hbar^{N}, \quad \hbar \in(0,1], \quad N \geq 0
$$


By Theorem A.24 we have for some $n \geq 0$ the estimate

$$
\left\|a_{\hbar}^{W}\left(y, D_{y}\right)\left(\mathcal{T}_{x / \hbar} \mathcal{M}_{\xi / \hbar} \psi_{0}\right)\right\|_{L^{2}} \lesssim\left\|\psi_{0}\right\|_{L^{2}} \sum_{|\alpha| \leq n} \hbar^{|\alpha|}\left\|\partial^{\alpha} a\right\|_{\infty} .
$$

This gives for $\hbar \in(0,1], \quad N \geq 0$

$$
\int_{U}\left|\mathscr{V}_{\hbar} u(x, \xi)\right|\left\|a_{\hbar}^{W}\left(y, D_{y}\right)\left(T_{x / \hbar} M_{\xi / \hbar} \psi_{0}\right)\right\|_{L^{2}} d x d \xi \lesssim \hbar^{N}
$$

It remains to estimate the integral over $\mathbb{R}^{2 d} \backslash U$ : since $u \in \mathscr{S}^{\prime}\left(\mathbb{R}^{d}\right)$, we have for some $M \in \mathbb{N}_{0}$ the polynomial bound

$$
\left|\mathscr{V}_{\hbar} u(x, \xi)\right| \lesssim h^{-d}\langle(x / \hbar, \xi / \hbar)\rangle^{M} \leq \hbar^{-d-M}\langle(x, \xi)\rangle^{M}, \quad \hbar \in(0,1] .
$$

Lemma 2.77 then yields for $a \in C_{c}^{\infty}\left(\mathbb{R}^{2 d}\right)$ supported in a suitably small neighbourhood of $\left(x_{0}, \xi_{0}\right), \hbar \in(0,1]$,

$$
\begin{aligned}
& \int_{\mathbb{R}^{2 d} \backslash U}\left|\mathscr{V}_{\hbar} u(x, \xi)\right|\left\|a_{\hbar}^{W}\left(y, D_{y}\right)\left(\mathcal{T}_{x / \hbar} \mathcal{M}_{\xi / \hbar} \varphi\right)\right\|_{L^{2}} d x d \xi \\
& \lesssim \hbar^{-d-M} \iint_{\mathbb{R}^{2 d} \backslash U}\langle(x, \xi)\rangle^{M} C_{N} \hbar^{N}\langle(x, \xi)\rangle^{-N / 2} d x d \xi \\
& \lesssim \hbar^{N-d-M},
\end{aligned}
$$

provided $N>2(M+2 d)$. Using (2.38) and (2.39) this shows that $\left(x_{0}, \xi_{0}\right) \notin$ $\operatorname{HWF}(u)$, which completes the proof of $\operatorname{HWF}(u) \subseteq \mathrm{WF}_{\mathrm{G}}(u)$ and thus of the Theorem.

2.6.5. A comparison between $\mathrm{WF}_{\mathrm{SG}}$ and $\mathrm{WF}_{\mathrm{G}}$.

We have already compared $\mathrm{WF}_{\mathrm{SG}}$ and $\mathrm{WF}_{\mathrm{G}}$ to some extent in the discussion following Example 2.59. Having Theorem 2.74 at hand, we may reformulate, using Fourier symmetry and the Sobolev spaces, some results that were given in [111, Prop. 1] for HWF in order to to compare $W_{G}$ and $W_{S G}$ further.

Proposition 2.78. Let $u \in \mathscr{S}^{\prime}\left(\mathbb{R}^{d}\right)$.

(1) Let $\xi_{0} \in \mathbb{R}^{d} \backslash\{0\}$. If $\left(0, \xi_{0}\right) \notin \mathrm{WF}_{\mathrm{G}}(u)$, then $\forall x \in \mathbb{R}^{d}$ we have $\left(x, \xi_{0}\right) \notin$ $\mathrm{WF}_{\mathrm{SG}}^{\psi}(u)$. In particular, if $(0, \xi) \notin \mathrm{WF}_{\mathrm{G}}(u)$ for all $\xi \in \mathbb{R}^{d} \backslash\{0\}$, then $u \in \mathscr{C}^{\infty}\left(\mathbb{R}^{d}\right)$.

(2) Let $x_{0} \in \mathbb{R}^{d} \backslash\{0\}$. If $\left(x_{0}, 0\right) \notin \mathrm{WF}_{\mathrm{G}}(u)$, then $\forall \xi \in \mathbb{R}^{d}$ we have $\left(x_{0}, \xi\right) \notin$ $\mathrm{WF}_{\mathrm{SG}}^{e}(u)$. In particular, if $(x, 0) \notin \mathrm{WF}_{\mathrm{G}}(u)$ for all $x \in \mathbb{R}^{d} \backslash\{0\}$, then $\widehat{u} \in \mathscr{C}^{\infty}\left(\mathbb{R}^{d}\right)$. 
(3) Let $x_{0} \in \mathbb{R}^{d} \backslash\{0\}$. If there is a cut-off $\phi^{x_{0} \infty}$ such that $\phi^{x_{0} \infty} u \in H_{\mathrm{SG}}^{\infty, 0}\left(\mathbb{R}^{d}\right)$, then $\forall \xi \in \mathbb{R}^{d}$ we have $\left(x_{0}, \xi\right) \notin \mathrm{WF}_{\mathrm{G}}(u)$. In particular, if $u \in H_{\mathrm{SG}}^{\infty, 0}\left(\mathbb{R}^{d}\right)$, then $\mathrm{WF}_{\mathrm{G}}(u) \subset\{0\} \times \mathbb{R}^{d} \backslash\{0\}$.

(4) Let $\xi_{0} \in \mathbb{R}^{d} \backslash\{0\}$. If there is a cut-off $\phi^{\xi_{0} \infty}$ such that $\phi^{\xi_{0} \infty} \widehat{u} \in H_{\mathrm{SG}}^{\infty, 0}\left(\mathbb{R}^{d}\right)$, then $\forall x \in \mathbb{R}^{d}$ we have $\left(x_{0}, \xi_{0}\right) \notin \mathrm{WF}_{\mathrm{G}}(u)$. In particular, if $u \in H_{\mathrm{SG}}^{0, \infty}\left(\mathbb{R}^{d}\right)$, then $\mathrm{WF}_{\mathrm{G}}(u) \subset \mathbb{R}^{d} \backslash\{0\} \times\{0\}$.

We remark that these results may be visualized in the time-frequency plane. We do so for (1) in Figure 11.

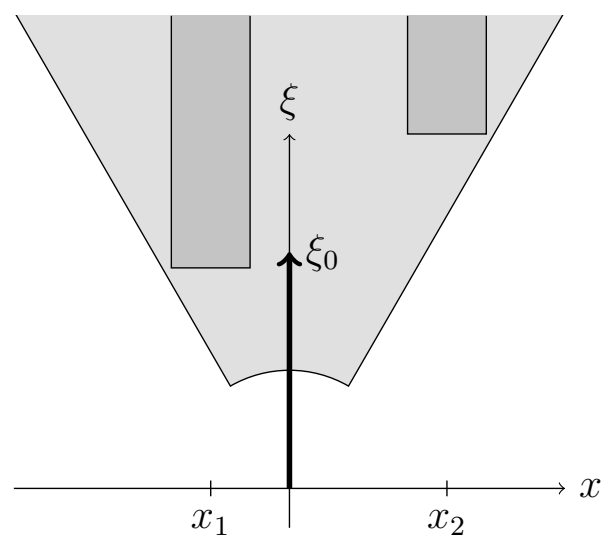

FigURE 11. Inclusion results of $\mathrm{WF}_{\mathrm{SG}}$ and $\mathrm{WF}_{\mathrm{G}}$.

The following example (see also [22]) underlines the usefulness of the $\mathrm{WF}_{\mathrm{G}}$ :

Example 2.79. Let $u_{0} \in \mathscr{S}^{\prime}\left(\mathbb{R}^{d}\right)$. Then the family of distributions

$$
\begin{aligned}
u:[0, \infty) & \rightarrow \mathscr{S}^{\prime}\left(\mathbb{R}^{d}\right) \\
t & \mapsto \mathcal{F}^{-1}\left(e^{i \xi^{2} t} \mathcal{F}\left(u_{0}\right)\right)
\end{aligned}
$$

is a solution to the homogeneous initial value problem for the Schrödinger equation

$$
\left\{\begin{aligned}
-i \partial_{t} u+\Delta u & =0 \\
\left.u\right|_{t=0} & =u_{0} .
\end{aligned}\right.
$$

By Lemma 2.56 we have

$$
(x, \xi) \in \mathrm{WF}_{\mathrm{G}}\left(u_{0}\right) \Leftrightarrow(x+t \xi, \xi) \in \mathrm{WF}_{\mathrm{G}}(u(t, \cdot)) .
$$


In particular this may be used to visualize (using of Proposition 2.78), with reference to the time-frequency plane, the well-known facts (see e.g. the introduction in [153]) that

- $u_{0}$ is compactly supported, then $u(t, \cdot)$ is smooth for all $t>0$,

- $u_{0}=e^{-\frac{i}{2} \lambda(\cdot)^{2}}$, then $u(t, \cdot)$ develops a singularity at $t=\frac{1}{\lambda}$.

For more on propagation of these kind of singularities under Schrödinger operators in various functional settings consider [30, 31, 85, 86, 100, 101, 109, 111, 153].

Remark 2.80. We mention that in [149], another global notion of singularity structure suitable for the analysis of Schrödinger equations, the metaplectic wave front set, MWF, is introduced with reference to yet another symbol class. It combines features of $\mathrm{WF}_{\mathrm{G}}(u)$ and $\mathrm{WF}_{\mathrm{SG}}(u)$ in that it contains the classical wave front set, but at the same time transforms adequately under the full metaplectically group. However, it has several draw-backs, which were already pointed out in [149], such as the facts that it is not defined as a closed set and does not possess the feature of global $\mathscr{S}$-regularity. 


\subsection{Operations on tempered distributions using $\mathrm{WF}_{\mathrm{G}}$.}

\subsubsection{Multilinear operations and composition with operators.}

It would be possible to produce the result about the pairing of distributions using the G-wave front set in a similar fashion as before in the SG-setting, i.e. Theorem 2.37

However, due to the simpler structure of $\mathrm{WF}_{\mathrm{G}}$, it is more convenient to obtain the result using pull-backs by linear maps and the tensor product of distributions, since these results take a much simpler form in the G-setting. This has already been achieved by Hörmander [82]. We present some of these results here to be able to use them for later applications and to be able to compare them with our findings in the SG-setting. For that, again, we first introduce the space of all distributions with wave front set in a given cone. By abuse of notation, we give it the same symbol as before, noting that this time $\Gamma$ is a subset of $W_{G}$ instead of $\mathrm{W}_{\mathrm{SG}}$.

Definition 2.81. Let $\Gamma \subset \mathrm{W}_{\mathrm{G}}$ closed subset. Define

$$
\mathscr{S}_{\Gamma}^{\prime}\left(\mathbb{R}^{d}\right)=\left\{u \in \mathscr{S}^{\prime}\left(\mathbb{R}^{d}\right) \mid \mathrm{WF}_{\mathrm{G}}(u) \subset \Gamma\right\}
$$

with a notion of convergence given by $u \stackrel{\mathscr{S}^{\prime}}{\rightarrow} 0$ if

(1) $u \stackrel{\mathscr{S}^{\prime}}{\rightarrow} 0$,

(2) for all $a \in G^{m}\left(\mathbb{R}^{d}\right)$ characteristic on all of $\Gamma$ we have $a^{W}(x, D) u \stackrel{\mathscr{S}}{\rightarrow} 0$.

Again, when we speak about sequential continuity in the following, we do so with reference to this notion of convergence. Once more we have denseness of $\mathscr{S}$ in $\mathscr{S}_{\Gamma}^{\prime}$, see [82]:

Lemma 2.82. $\mathscr{S}\left(\mathbb{R}^{d}\right)$ is dense in $\mathscr{S}_{\Gamma}^{\prime}\left(\mathbb{R}^{d}\right)$.

By analysing the elementary maps $\iota_{d}: \mathbb{R}^{d} \rightarrow \mathbb{R}^{d+1}, \mathrm{pr}_{d}: \mathbb{R}^{d+1} \rightarrow \mathbb{R}^{d}$ and $\mathcal{D}_{A}$ of Section 2.4.1. Hörmander 82] obtains:

Theorem 2.83 (Behaviour of $\mathrm{WF}_{\mathrm{G}}$ under pull-backs by linear maps). Let $A$ be $a$ linear map $\mathbb{R}^{d} \rightarrow \mathbb{R}^{s}, \Gamma \subset \mathrm{W}_{\mathrm{G}}$ closed. Then for all $u \in \mathscr{S}_{\Gamma}^{\prime}\left(\mathbb{R}^{d}\right)$ such that

$$
\Gamma \cap\left\{(0, \xi) \mid{ }^{t} A \xi=0\right\}=\emptyset
$$


the pull-back $A^{*} u$ can be uniquely defined, such that it is a sequentially continuous map from $\mathscr{S}_{\Gamma}^{\prime}\left(\mathbb{R}^{s}\right)$ to $\mathscr{S}^{\prime}\left(\mathbb{R}^{d}\right)$. In fact we have the inclusion

$$
\mathrm{WF}_{\mathrm{G}}\left(A^{*} u\right) \subset A^{*} \mathrm{WF}_{\mathrm{G}}(u)=\left\{\left(x,{ }^{t} A \xi\right) \mid(A x, \xi) \in \mathrm{WF}_{\mathrm{G}}(u)\right\} .
$$

We further have the following behaviour under tensor products

Lemma 2.84 (Behaviour of $\mathrm{WF}_{\mathrm{G}}$ under tensor products). Let $u_{i} \in \mathscr{S}^{\prime}\left(\mathbb{R}^{d_{i}}\right)$. Then we have

$$
\mathrm{WF}_{\mathrm{G}}\left(u_{1} \otimes u_{2}\right) \subset\left\{\left(x_{1}, x_{2} ; \xi_{1}, \xi_{2}\right) \mid\left(x_{i}, \xi_{i}\right) \in\left(\mathrm{WF}_{\mathrm{G}}\left(u_{i}\right) \cup\{0\}\right)\right\} \backslash\{0\}
$$

Remark 2.85. Both of the previous results can be proved in two ways: either by pseudo-differential calculations, or, in a more straight-forward calculation-based approach, by use of Proposition 2.60 and by calculating $\mathscr{V}_{\psi_{0}}\left(\operatorname{pr}_{d}^{*} u\right), \mathscr{V}_{\psi_{0}}\left(\iota_{d}^{*} u\right)$, $\mathscr{V}_{\psi_{0}}\left(A^{*} u\right)$ and $\mathscr{V}_{\psi_{0}}\left(u_{1} \otimes u_{2}\right)$. The latter is particularly easy, since

$$
\mathscr{V}_{\psi_{0}}\left(u_{1} \otimes u_{2}\right)=\mathscr{V}_{\psi_{0}}\left(u_{1}\right) \otimes \mathscr{V}_{\psi_{0}}\left(u_{2}\right),
$$

while it is quite complicated for pseudo-differential operators, see [82, Prop. 2.8], since 36

$$
\mathrm{G}^{0}\left(\mathbb{R}^{d} \times \mathbb{R}^{d}\right) \otimes \mathrm{G}^{0}\left(\mathbb{R}^{d^{\prime}} \times \mathbb{R}^{d^{\prime}}\right) \not \subset \mathrm{G}^{0}\left(\mathbb{R}^{d+d^{\prime}} \times \mathbb{R}^{d+d^{\prime}}\right) .
$$

With Theorem 2.83 and Lemma 2.84, we are once more able to characterize the product of two tempered distributions in terms of the diagonal embedding $\delta$ via $\delta^{*}(u \otimes v)$. Hörmander obtained the pairing from the fact that $\mathcal{F}\{f\}(0)=$ $\int_{\mathbb{R}^{d}} f(x) d x$ and thus by applying Fourier transformation and the pull-back by $0 \hookrightarrow \mathbb{R}^{2 d}$ to $u \otimes v$. Alternatively (to avoid the Fourier transform) it is possible to repeat the construction of 2.37 for the G-calculus. We summarize the two operations in the following Proposition. We do not give a proof here, since the pairing is already covered in [82]. A proof for the product, which is not treated in [82], is a special case of the construction in Section 3.4 .

Proposition 2.86. Let $u_{1}, u_{2} \in \mathscr{S}^{\prime}\left(\mathbb{R}^{d}\right)$. Then

(1) If $(0, \xi) \in \mathrm{WF}_{\mathrm{G}}\left(u_{1}\right) \Rightarrow(0,-\xi) \notin \mathrm{WF}_{\mathrm{G}}\left(u_{2}\right)$, then their product is welldefined. The $\mathrm{G}$-wave front set of the product is contained in

$$
\mathrm{WF}_{\mathrm{G}}\left(u_{1}\right) \cup \mathrm{WF}_{\mathrm{G}}\left(u_{2}\right) \cup\left\{\left(x, \xi_{1}+\xi_{2}\right) \mid\left(x, \xi_{i}\right) \in \mathrm{WF}_{\mathrm{G}}\left(u_{i}\right)\right\} .
$$

\footnotetext{
${ }^{36}$ Recall that we have already used this to overcome the same difficulties in the SG-setting.
} 
The product map is a sequentially continuous map

$$
\mathscr{S}_{\mathrm{WF}_{\mathrm{G}}\left(u_{1}\right)}^{\prime}\left(\mathbb{R}^{d}\right) \times \mathscr{S}_{\mathrm{WF}_{\mathrm{G}}\left(u_{2}\right)}^{\prime}\left(\mathbb{R}^{d}\right) \rightarrow \mathscr{S}^{\prime}\left(\mathbb{R}^{d}\right) .
$$

(2) If $(x, \xi) \in \mathrm{WF}_{\mathrm{G}}\left(u_{1}\right) \Rightarrow(x,-\xi) \notin \mathrm{WF}_{\mathrm{G}}\left(u_{2}\right)$, then their pairing is welldefined. The pairing is a sequentially continuous map

$$
\mathscr{S}_{\mathrm{WF}_{\mathrm{G}}\left(u_{1}\right)}^{\prime}\left(\mathbb{R}^{d}\right) \times \mathscr{S}_{\mathrm{WF}_{\mathrm{G}}\left(u_{2}\right)}^{\prime}\left(\mathbb{R}^{d}\right) \rightarrow \mathbb{R}^{d}
$$

Remark 2.87. Again, Proposition 2.86 enables us to define convolutions as well.

This can be used to define composition with operators in terms of their kernels, by revisiting (2.21), as in [82, Prop. 2.11]:

Proposition 2.88 (Composition with operators). Let $u \in \mathscr{S}^{\prime}\left(\mathbb{R}^{d}\right)$ and $\mathcal{K}_{A} \in$ $\mathscr{S}^{\prime}\left(\mathbb{R}^{d^{\prime}+d}\right)$ the Schwartz kernel of an operator $A: \mathscr{S}\left(\mathbb{R}^{d}\right) \rightarrow \mathscr{S}^{\prime}\left(\mathbb{R}^{d^{\prime}}\right)$. Let

$$
\begin{aligned}
\mathrm{WF}_{\mathrm{G}}^{\prime}\left(\mathcal{K}_{A}\right) & =\left\{(x, y ; \xi,-\eta) \mid(x, y ; \xi, \eta) \in \mathrm{WF}_{\mathrm{G}}\left(\mathcal{K}_{A}\right)\right\} \\
\operatorname{pr}_{1}^{0} \mathrm{WF}_{\mathrm{G}}^{\prime}\left(\mathcal{K}_{A}\right) & =\left\{(x, \xi) \mid(x, 0, \xi, 0) \in \mathrm{WF}_{\mathrm{G}}^{\prime}\left(\mathcal{K}_{A}\right)\right\} \\
\operatorname{pr}_{2}^{0} \mathrm{WF}_{\mathrm{G}}^{\prime}\left(\mathcal{K}_{A}\right) & =\left\{(y, \eta) \mid(0, y, 0, \eta) \in \mathrm{WF}_{\mathrm{G}}^{\prime}\left(\mathcal{K}_{A}\right)\right\}
\end{aligned}
$$

Then we have, for $f \in \mathscr{S}\left(\mathbb{R}^{d}\right)$,

$$
\mathrm{WF}_{\mathrm{G}}(A f) \subset \operatorname{pr}_{1}^{0}\left(\mathrm{WF}_{\mathrm{G}}^{\prime}\left(\mathcal{K}_{A}\right)\right) .
$$

More generally, A may be extended in a canonical fashion to all $u \in \mathscr{S}^{\prime}\left(\mathbb{R}^{d}\right)$ with

$$
\mathrm{WF}_{\mathrm{G}}(u) \cap \operatorname{pr}_{2}^{0}\left(\mathrm{WF}_{\mathrm{G}}^{\prime}\left(\mathcal{K}_{A}\right)\right)=\emptyset .
$$

For those we have

$$
\mathrm{WF}_{\mathrm{G}}(A u) \subset \operatorname{pr}_{1}^{0}\left(\mathrm{WF}_{\mathrm{G}}^{\prime}\left(\mathcal{K}_{A}\right)\right) \cup\left(\mathrm{WF}_{\mathrm{G}}^{\prime}\left(\mathcal{K}_{A}\right) \circ \mathrm{WF}_{\mathrm{G}}(u)\right),
$$

where $\mathrm{WF}_{\mathrm{G}}^{\prime}\left(\mathcal{K}_{A}\right)$, the $\mathrm{G}$-wavefront relation is regarded as a set theoretical relation $\mathrm{WF}_{\mathrm{G}}^{\prime}\left(\mathcal{K}_{A}\right): T^{*} \mathbb{R}^{d} \rightarrow T^{*} \mathbb{R}^{d^{\prime}}$.

\subsubsection{Affine maps.}

To conclude this section, one might again study affine maps. In the G-setting these are treated in a simple Lemma:

Lemma 2.89. For $x_{0}, \xi_{0} \in \mathbb{R}^{d}$ we have

$$
\mathrm{WF}_{\mathrm{G}}(u)=\mathrm{WF}_{\mathrm{G}}\left(\mathcal{T}_{x_{0}} u\right)=\mathrm{WF}_{\mathrm{G}}\left(\mathcal{M}_{\xi_{0}} u\right)
$$


Proof. This was concluded in [121] from the fact that translations and modulations may be written as pseudo-differential operators with symbol in $G_{0}^{0}$ and by applying Proposition 2.66. It may also be obtained from the characterization of $\mathrm{WF}_{\mathrm{G}}$ in terms of the STFT and A.9.

Thus finite translations are not "seen" by $\mathrm{WF}_{\mathrm{G}}$. One may, however, apply Theorem 2.52 and Lemma 2.84 to characterize the wave front set of translationinvariant distributions. 


\section{Special constructions and applications}

\subsection{Outline.}

On a formal level, the SG- and G-wave front sets are defined in a very similar manner, but we have already seen that they differ quite significantly in some details. The main features that separate them are their different resolutions of the time-frequency plane:

- $\mathrm{WF}_{\mathrm{SG}}$ contains local information, in particular $\operatorname{pr}_{1}\left(\mathrm{WF}_{\mathrm{SG}}\right)(u)=\operatorname{Css}(u)$ and $\mathrm{WF}_{\mathrm{SG}}^{\psi}=\mathrm{WF}_{\mathrm{cl}}$.

- $\mathrm{WF}_{\mathrm{G}}$, however, transforms in a known way under symplectic transformations, but is not as "localizable".

We will take advantage of these features during the next sections, for which we indicate the following outline.

- The property of $\mathrm{WF}_{\mathrm{SG}}^{\psi}=\mathrm{WF}_{\mathrm{cl}}$ enables us to ask how constructions involving the classical wave front set can be "globalized" to such involving $\mathrm{WF}_{\mathrm{SG}}$. Some of the most important applications of microlocal methods are within the theory of Fourier integral operators and Lagrangian distributions, see Hörmander [79]. Oscillatory integrals, which (micro-)locally represent these objects, form a class of distributions whose classical wave front sets are well-understood. In fact the arising wave front sets are of a special type, namely (conic) Lagrangian submanifolds known from symplectic geometry, parametrised in terms of the phase functions associated to the corresponding families of oscillatory integrals. Conversely, all such conic Lagrangian submanifolds can (locally) be parametized by some phase function and give rise to a class of distributions.

We may therefore ask if such a theory has a generalization to the tempered SG-microlocal setting. This is discussed in Section 3.2. We give a brief summary of the classical theory in Section 3.2.1. In Section 3.2.2 and 3.2.3 we present results, obtained in collaboration with Coriasco [49], on how to define tempered oscillatory integrals and how their singularities are bounded by the stationary phase points of the associated phase function. Continuing this analysis with recent results from our ongoing collaboration [49], in Section 3.2.4, we study the underlying geometry "at infinity". In accordance with the classical theory, we characterize, for certain non-degenerate 
SG-phase functions, the aforementioned geometric bounds on the SG-wave front sets as SG-Lagrangians. We also obtain a a converse characterization, meaning all such SG-Lagrangians are again locally parametrizable by non-degenerate SG-phase functions.

- We have already seen how dilations, i.e. scaling of distributions, may be used to obtain (microlocal) information about this distribution. It is also easy to see that the scaling behaviour of a distribution is linked to its order. In Section 3.3.1, we follow the ideas of Weinstein [148] to discuss how these considerations may be used to give a notion of "microlocal order" at a point and how to define the symbol of a tempered distribution.

In Section 3.3.2, bounds on the scaling behaviour of a distribution are used to extend distributions defined outside of a subspace to the whole space.

- In Section 3.4, the train of thought of Section 2.7.1 is continued, taking into account the full symplectic invariance of $\mathrm{WF}_{\mathrm{G}}$ to study, G-microlocally, the Weyl product and Weyl quantization of distributions.

- In Section 3.5 we discuss how our previous techniques may be employed to define certain distributions arising in the perturbative approach to axiomatic constructive quantum field theory. In particular, we discuss the two-point function as a tempered oscillatory integral and discuss its singularities in Section 3.5.2. We use this to define products with other distributions as well as (twisted) powers of it in Section 3.5.3. 


\subsection{Tempered oscillatory integrals.}

\subsubsection{Introduction and elements of the classical theory.}

Oscillatory integrals arise in many subjects of mathematics and physics. They take the form

$$
I_{\varphi}(a)=\int_{\mathbb{R}^{s}} e^{i \varphi(x, \theta)} a(x, \theta) d \theta,
$$

where $\varphi$ is called the phase function and $a$ is called the amplitude. Even if the integral does not converge to a function in $x$, we are, under the right assumptions on $\varphi$ and $a$, able to treat these objects as distributions, meaning via their action

$$
f \mapsto \iint_{X \times \mathbb{R}^{s}} e^{i \varphi(x, \theta)} a(x, \theta) f(x) d \theta d x
$$

Oscillatory integrals were studied by Hörmander as the (micro-)local representations of Lagrangian distributions in [79], see also [55, 56, 67, 81, 83, 84, 145]. These distributions arise in particular as kernels of Fourier integral operators (FIO), which often appear as solution operators to hyperbolic partial differential equations. His theory is well suited to study problems in open neighbourhoods of $\mathbb{R}^{d}$ and compact manifolds. In order to treat global problems on non-compact spaces (or on their compactifications, that is spaces with boundaries) it is necessary to impose bounds on these distributions, such as temperedness. This translates to studying objects of the form (3.1) where we impose bounds on the (derivatives of the) symbols and phase functions. Several authors have adapted to work with SGor G-symbols ${ }^{37}$ We are going to consider the SG-setting here.

While the subject of $L^{p}$-continuity of Fourier integral operators on $\mathbb{R}^{d}$ has been studied in many global classes of FIOs, see e.g. [2], [3], [32, 33] and [123], to the best of our knowledge propagation of SG-singularities were only studied in the approach using SG-Fourier Integral operators on $\mathbb{R}^{d}$ of Coriasco ([38, 39, 44, 45, 46], see also [1]) and in the formalism of Legendrian distributions on scattering manifolds, by Melrose and Zworski [102], see also [107, 70, 71]. We also note that in recent works of Battisti, Coriasco and Schrohe [7, 8], a Boutet de Monvel-type calculs of FIOs associated to boundary-preserving symplectomorphisms is established, which is also suitable for the study of propagation of singularities, but does

\footnotetext{
$\overline{37}$ The Fourier Integral operators studied that may be represented by quadratic phase functions and admit G-symbols are often so-called "Metaplectic operators", see e.g. [30, 31, 32, 33, 34.
} 
not allow for propagation into and out of the boundary.

In the following we will present a theory of generalized oscillatory integrals and Lagrangians as developed in collaboration with Coriasco [49, 50]. The advantage of our approach is that we are able to formulate it in terms manifestly similar to the classical ones, while still admitting a broad class of phase functions that give rise to "singularities at infinity". In particular we allow for corner-type, that is $\psi e$-contributions to the wave front set 38

In order to properly motivate the upcoming results and definitions and for the convenience of the reader, we are first going to recall some elements of the classical theory of oscillatory integrals and Lagrangian distributions.

In the following, let $X \subset \mathbb{R}^{d}$. We begin by introducing classical (homogeneous) phase functions $\varphi$, see [81, Sect. 7.8.] and [67, Def. 1.10]. These are elements of $\mathscr{C}^{\infty}\left(X \times\left(\mathbb{R}^{s} \backslash\{0\}\right)\right)$ such that for all $(x, \theta) \in X \times\left(\mathbb{R}^{s} \backslash\{0\}\right)$ we have

- $\Im \varphi \geq 0$,

- $\varphi(x, \lambda \theta)=\lambda \varphi(x, \theta)$,

- $d \varphi \neq 0$.
(Positivity of the imaginary part)

(Homogeneity)

(Absence of critical points)

The following Theorem, see [79, Prop. 1.2.2], allows us to then define oscillatory integrals as distributions.

Theorem 3.1. Let $\varphi$ a phase function. Then $\sqrt{3.2}$ can be extended ${ }^{39}$ in a unique way to all Hörmander symbols a such that $a \mapsto I_{\varphi}(a) \in \mathscr{D}^{\prime}\left(\mathbb{R}^{d}\right)$ is linear and continuou\& in a.

${ }^{38}$ This is the main difference with respect to the previously mentioned approaches:

- In [38, see also [1, the phase functions (or phase components) under consideration fulfil

$$
\begin{aligned}
\left\langle\nabla_{x} \varphi(x, \theta)\right\rangle & \asymp\langle\theta\rangle \\
\left\langle\nabla_{\theta} \varphi(x, \theta)\right\rangle & \asymp\langle x\rangle
\end{aligned}
$$

which enforces, by the upcoming analysis, $\operatorname{Css}\left(I_{\varphi}(a)\right) \subset\{0\}$.

- The singularities of the Fourier transforms of Legendrian distributions on Euclidean spaces are contained in compact sets, by [107, Prop. 10], a feature that is not true for our class of distributions.

\footnotetext{
${ }^{39}$ The expression is obviously well-defined if $a \in \mathscr{S}\left(\mathbb{R}^{d} \times \mathbb{R}^{s}\right)$ and $u \in \mathscr{D}\left(\mathbb{R}^{d}\right)$.

${ }^{40}$ Continuity is meant with respect to the Frechét topology on the space of all Hörmander symbols.
} 
Consequently we have, for any phase function, an associated family of distributions. We will now turn to the characterization of the classical singularities of such distributions, which are bounded by geometric objects associated to the phase function.

To any phase function one associates the set

$$
\mathcal{C}_{\varphi}:=\left\{(x, \theta) \in X \times\left(\mathbb{R}^{s} \backslash\{0\}\right) \mid \nabla_{\theta} \varphi=0\right\}
$$

and the map

$$
\lambda_{\varphi}: X \times \mathbb{R}^{s} \backslash\{0\} \rightarrow X \times \mathbb{R}^{d} \quad(x, \theta) \mapsto\left(x, \nabla_{x} \varphi(x, \theta)\right) .
$$

By the homogeneity assumption on $\varphi, \mathcal{C}_{\varphi}$ is conic in the second set of variables. By the assumption on the absence of critical points, we have that $\lambda_{\varphi}$ maps $\mathcal{C}_{\varphi} \rightarrow$ $X \times\left(\mathbb{R}^{d} \backslash\{0\}\right)$ and the conic (in the covariable) target set

$$
\Lambda_{\varphi}:=\lambda_{\varphi}\left(\mathcal{C}_{\varphi}\right)=\left\{\left(x, \nabla_{x} \varphi(x, \theta)\right) \mid(x, \theta) \in \mathcal{C}_{\varphi}\right\}
$$

is called the set of stationary points associated to $\varphi$. Then the following inclusion (see [55, Thm. 2.2.2], [79, Prop. 2.5.7.], [81, Thm. 8.1.9], [67, Chap. 7]) holds:

Theorem 3.2. For the distribution defined in Theorem 3.1 we have

$$
\begin{aligned}
\operatorname{singsupp}\left(I_{\varphi}(a)\right) & \subset \operatorname{pr}_{1}\left(\mathcal{C}_{\varphi}\right) \\
\operatorname{WF}_{\mathrm{cl}}\left(I_{\varphi}(a)\right) & \subset \Lambda_{\varphi} .
\end{aligned}
$$

A phase function is called non-degenerate, see [83, Def. 21.2.15.], if

- $\mathcal{C}_{\varphi}$ is a $d$-dimensional $\mathscr{C}^{\infty}$-manifold with tangent plane defined by the equations $d\left(\nabla_{\theta} \varphi\right)=0$ and the differentials $\left\{d_{(x, \theta)} \partial_{\theta_{j}} \varphi(x, \theta)\right\}_{j \in\{1 . . s\}}$ are linearly independent.

(Non-degeneracy)

The symplectic properties of manifolds of the form $\Lambda_{\varphi}$ are recalled in the following result (see e.g. [55, Prop 3.7.3.])

Proposition 3.3. For a non-degenerate phase function $\varphi$ on $X \times\left(\mathbb{R}^{s} \backslash\{0\}\right)$, the set of stationary points $\Lambda_{\varphi}$ is an immersed conic Lagrangian submanifold of $T^{*} X$, which means $\Lambda_{\varphi}$ is a $\mathscr{C}^{\infty}$-manifold of dimensiond that satisfies $\left.\alpha^{\psi}\right|_{\Lambda_{\varphi}} \equiv 0$, where $\alpha^{\psi}$ is the tautological one-form on $T^{*} X$.

We then also have the converse characterization, see e.g. Theorem 21.2.16. and 21.2.17. in [83]: 
Theorem 3.4. Let $\Lambda \subset T^{*} \mathbb{R}^{d} \backslash\{0\}$ a conic Lagrangian submanifold.

Let $z_{0}=\left(x_{0}, \xi_{0}\right) \in \Lambda$ and $\operatorname{dim}\left(T_{z_{0}} \Lambda \cap T_{x_{0}}^{*} \mathbb{R}^{d}\right)=s$. Then there exists a neighbourhood $U$ of $x_{0}$, a cone $\Gamma \subset\left(\mathbb{R}^{s} \backslash\{0\}\right)$ and a non-degenerate phase function $\varphi \in \mathscr{C}^{\infty}(U \times \Gamma){ }^{41}$ such that $\Lambda$ can be locally parametrized as $\Lambda_{\varphi}$ in the sense of (3.5).

The following Theorem, [79, Thm. 6], guarantees the existence of a diffeomorphism mapping two such phase functions $\varphi_{1}, \varphi_{2}$ into each other, which means that they are equivalent.

Theorem 3.5. Two phase functions $\varphi_{1}$ and $\varphi_{2}$ in conic neighbourhoods of $\left(x_{0}, \theta_{0,1}\right) \in \mathbb{R}^{d} \times \mathbb{R}^{s}$ and $\left(x_{0}, \theta_{0,2}\right) \in \mathbb{R}^{d} \times \mathbb{R}^{s}$ respectively are equivalent in some conic neighbourhood of these points, under a (local) diffeomorphism mapping $\left(x_{0}, \theta_{0,1}\right)$ to $\left(x_{0}, \theta_{0,2}\right)$, if and only if

- $\varphi_{1}$ and $\varphi_{2}$ locally parametrize the same Lagrangian,

- $\left(\partial_{\theta_{j}} \partial_{\theta_{k}} \varphi_{1}\left(x_{0}, \theta_{0,1}\right)\right)_{j, k}$ and $\left(\partial_{\theta_{j}} \partial_{\theta_{k}} \varphi_{2}\left(x_{0}, \theta_{0,2}\right)\right)_{j, k}$ have the same signature.

We conclude this brief recap of the classical theory by coming back to the initial oscillatory integrals and how they relate to the previous geometric objects. We have recalled how any (non-degenerate) phase function gives rise to an associated family of distributions and a conic Lagrangian. Conversely, any conic Lagrangian gives rise (locally) to a phase function. The so-called Lagrangian distributions (or Lagrangian distributional densities) associated to $\Lambda$ are then those distributions that are (micro-)locally given by oscillatory integrals for phase functions locally parametrizing $\Lambda$, see [79, Def. 3.2.2.].

In the following we will now present our findings in [49, 50], which generalize the previous notions to the global setting on $\mathbb{R}^{d}$ in an outline as above ${ }^{42}$ We first define tempered oscillatory integrals and study their singularities and then discuss the geometric objects associated to the class of phase functions used in the analysis.

\subsubsection{Definition of tempered oscillatory integrals.}

We begin by introducing a very broad class of SG-phase functions. We focus on real-valued phase functions, the extension to such with positive imaginary part is straight-forward.

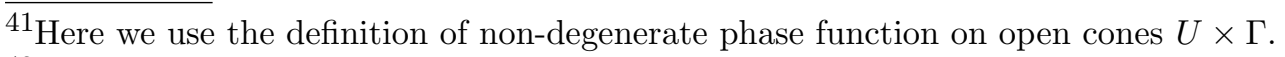

${ }^{42}$ For that, as in [50], we have updated our notation with respect to [49] to a notation closer to [79, 83, for the convenience of the reader.
} 
Remark 3.6. In the following, we generalize the notion of $\mathrm{SG}^{m_{e}, m_{\psi}}$ to $\mathbb{R}^{d} \times \mathbb{R}^{s}$, which is a straight-forward modification of the definitions in Section 1.2.3. In particular, we have $\mathrm{SG}_{\mathrm{cl}}^{m_{e}, m_{\psi}}\left(\mathbb{R}^{d} \times \mathbb{R}^{s}\right) \cong \mathrm{S}_{\mathrm{cl}}^{m_{e}}\left(\mathbb{R}^{d}\right) \otimes \mathrm{S}_{\mathrm{cl}}^{m_{\psi}}\left(\mathbb{R}^{s}\right)$.

Definition 3.7. Let $\left(n_{e}, n_{\psi}\right) \in \mathbb{R}_{+}^{2}$. An admissible inhomogeneous SG-phase function of order $\left(n_{e}, n_{\psi}\right)$ is a real-valued element of $\mathrm{SG}^{n_{e}, n_{\psi}}\left(\mathbb{R}^{d} \times \mathbb{R}^{s}\right)$ such that

$$
\Phi(x, \theta):=\langle x\rangle^{2}\left|\nabla_{x} \varphi(x, \theta)\right|^{2}+\langle\theta\rangle^{2}\left|\nabla_{\theta} \varphi(x, \theta)\right|^{2},
$$

as an element of $\mathrm{SG}^{2 n_{e}, 2 n_{\psi}}\left(\mathbb{R}^{d} \times \mathbb{R}^{s}\right)$, is elliptic, i.e. satisfies for some $R>0$

$$
\Phi(x, \theta) \gtrsim\langle x\rangle^{2 n_{e}}\langle\theta\rangle^{2 n_{\psi}} \text { when }|x|+|\theta| \geq R
$$

Remark 3.8. We note that these SG-phase functions do not form a subset of the classical phases that occurred in Section 3.2.1, since the are in general inhomogeneous. It has been already remarked by Hörmander [79], see also [67], that the assumption of homogeneity may be relaxed. Indeed our theory is based on the works of [156], where a local theory of oscillatory integrals with inhomogeneous phase functions was developed.

Indeed, there is a connection to the classical theory: assuming $n_{\psi}=1$ and that $\varphi$ admits a homogeneous symbol $\varphi^{\psi}:=\sigma^{\psi}(\varphi)$ such that $\varphi-(\mathbb{1} \otimes \chi) \varphi^{\psi} \in \mathrm{SG}^{n_{e}, 0}$ for any excision function $\chi$, we can write in $3.2{ }^{43}$

$$
e^{i \varphi(x, \theta)}=\underbrace{e^{i \varphi^{\psi}(x, \theta)}}_{\text {Cl. phase }} \cdot \underbrace{\chi(\theta) e^{i\left(\varphi-\varphi^{\psi}\right)(x, \theta)}}_{\text {Cl. symbol }}+\underbrace{(1-\chi(\theta)) e^{i \varphi(x, \theta)}}_{\text {Compactly supported in } \theta} .
$$

Therefore relaxing the assumption of homogeneity to asymptotic homogeneity is not a big change. Our assumption on the phase function being smooth everywhere, even at $\theta=0$, however, is an important feature during the upcoming analysis.

Using the notion of an admissible phase function, we are now ready to make sense of the formal expression $(3.2)$ as a tempered distribution.

Theorem 3.9. With any fixed admissible inhomogeneous SG-phase function $\varphi$ of order $\left(n_{e}, n_{\psi}\right)$ we may associate a map

$$
I_{\varphi}: \mathrm{SG}\left(\mathbb{R}^{d} \times \mathbb{R}^{s}\right) \rightarrow \mathscr{S}^{\prime}\left(\mathbb{R}^{d}\right)
$$

\footnotetext{
$\overline{43}$ This can be compared with the treatment of Example 7 in [118, Sect. IX.10], wherein the phase of the two-point function was similarly "homogeneized", which we will discuss in more detail in Section 3.5 .2
} 
uniquely determined by the the following properties:

(1) $a \mapsto I_{\varphi}(a)$ is a linear map;

(2) If $a \in \mathscr{S}\left(\mathbb{R}^{d} \times \mathbb{R}^{s}\right)$, then $I_{\varphi}(a)$ coincides with the (absolutely convergent) integral (3.1);

(3) the restriction of $I_{\varphi}$ to $\mathrm{SG}^{m_{e}, m_{\psi}}\left(\mathbb{R}^{d} \times \mathbb{R}^{s}\right)$ is a continuous map

$$
\mathrm{SG}^{m_{e}, m_{\psi}}\left(\mathbb{R}^{d} \times \mathbb{R}^{s}\right) \rightarrow \mathscr{S}^{\prime}\left(\mathbb{R}^{d}\right) .
$$

We call the resulting distribution $I_{\varphi}(a)$ a SG-oscillatory integral.

We follow our proof of [49, Thm. 3.1], which in turn is based on the classical proof in [79, Sect. 1.2] with the adaptations for inhomogeneous phase functions of [156, Thm. 1] ${ }^{44}$ It essentially breaks down into two parts: one is to prove the existence of an operator associated to the phase function that can be used to regularize the integral (3.2) by suitably reducing the symbol order of the amplitude. The second is to prove that this procedure may be used to continuously extend $(3.2)$ in $\mathscr{S}^{\prime}$ even for amplitudes for which the integral does not converge.

Lemma 3.10. Let $\varphi$ be a given admissible inhomogeneous SG-phase function of order $\left(n_{e}, n_{\psi}\right)$. Then there exists

$$
\begin{aligned}
& b_{j} \in \mathrm{SG}^{-n_{e}+1,-n_{\psi}}\left(\mathbb{R}^{d} \times \mathbb{R}^{s}\right) \quad j \in\{1, \ldots, s\}, \\
& c_{k} \in \mathrm{SG}^{-n_{e},-n_{\psi}+1}\left(\mathbb{R}^{d} \times \mathbb{R}^{s}\right) \quad k \in\{1, \ldots, d\}, \\
& d \in \mathrm{SG}^{-n_{e},-n_{\psi}}\left(\mathbb{R}^{d} \times \mathbb{R}^{s}\right),
\end{aligned}
$$

such that the linear differential operator

$$
P=b \cdot \nabla_{\theta}+c \cdot \nabla_{x}+d,
$$

with (formal) transpose (with respect to $f \in \mathscr{S}\left(\mathbb{R}^{d} \times \mathbb{R}^{s}\right) \subset L^{2}\left(\mathbb{R}^{d} \times \mathbb{R}^{s}\right)$ )

$$
{ }^{t} P f=-\nabla_{\theta} \cdot(b f)-\nabla_{x} \cdot(c f)+d f
$$

satisfies

$$
{ }^{t} P e^{i \varphi(x, \theta)}=e^{i \varphi(x, \theta)} .
$$

\footnotetext{
${ }^{44}$ For further versions following the same idea, see [55, Thm. 2.2.1.], 67, Thm. 1.1] and [118, Thm. IX.47]. The proof in [81, Thm. 8.1.9] makes use of the stationary phase lemma. Since we consider unbounded sets and need global bounds, we do not that approach here, since we do not restrict our analysis to classical SG-symbols, where we could take advantage of the isomorphism of Proposition 1.20 .
} 
Furthermore, $P$ is a continuous map

$$
P: \mathrm{SG}^{m_{e}, m_{\psi}}\left(\mathbb{R}^{d} \times \mathbb{R}^{s}\right) \rightarrow \mathrm{SG}^{m_{e}-n_{e}, m_{\psi}-n_{\psi}}\left(\mathbb{R}^{d} \times \mathbb{R}^{s}\right) .
$$

Proof. The proof coincides in its essentials with that of our proof of [49, Lem. 4] and is basically a SG-variant of the one from [156, Lem. 2.10].

Consider the SG-symbol $\Phi$ associated to $\varphi$ introduced in (3.6) and take an excision function $\chi(x, \theta)=\left(1-\phi^{0}\right)(x, \theta)$ in $\mathrm{SG}^{0,0}\left(\mathbb{R}^{d} \times \mathbb{R}^{s}\right)$ such that on the support of $\chi$ we have

$$
|\Phi(x, \theta)| \gtrsim\langle x\rangle^{2 n_{e}}\langle\theta\rangle^{2 n_{\psi}},
$$

by taking $\chi \equiv 1$ for $|x|+|\theta|>R+1$ and $\chi \equiv 0$ for $|x|+|\theta| \leq R$ with respect to the $R$ in (3.6).

Then $\chi \Phi^{-1} \in \mathrm{SG}^{-2 n_{e},-2 n_{\psi}}\left(\mathbb{R}^{d} \times \mathbb{R}^{s}\right)$ and we can set

$$
\begin{aligned}
b_{j}(x, \theta) & =i\left(\chi \Phi^{-1}\right)(x, \theta)\langle\theta\rangle^{2} \partial_{\theta_{j}} \varphi(x, \theta) \in \mathrm{SG}^{-n_{e}+1,-n_{\psi}}\left(\mathbb{R}^{d} \times \mathbb{R}^{s}\right), \\
c_{k}(x, \theta) & =i\left(\chi \Phi^{-1}\right)(x, \theta)\langle x\rangle^{2} \partial_{x_{k}} \varphi(x, \theta) \in \mathrm{SG}^{-n_{e},-n_{\psi}+1}\left(\mathbb{R}^{d} \times \mathbb{R}^{s}\right), \\
d(x, \theta) & =\left(\nabla_{\theta} \cdot b+\nabla_{x} \cdot c+\phi^{0}\right)(x, \theta) \in \mathrm{SG}^{-n_{e},-n_{\psi}}\left(\mathbb{R}^{d} \times \mathbb{R}^{s}\right) .
\end{aligned}
$$

Using integration by parts (note that the boundary terms vanish, since we consider the transpose with respect to $\left.\mathscr{S}\left(\mathbb{R}^{d} \times \mathbb{R}^{s}\right)\right)$, it is easy to verify that the operator ${ }^{t} P$ defined in $(3.10)$ is indeed the transpose of $P$ with respect to $\mathscr{S}\left(\mathbb{R}^{d} \times \mathbb{R}^{s}\right)$. One verifies (3.11) by calculating

$$
\begin{aligned}
{ }^{t} P e^{i \varphi} & =-\nabla_{\theta} \cdot\left(b e^{i \varphi}\right)-\nabla_{x} \cdot\left(c e^{i \varphi}\right)+d e^{i \varphi} \\
& =\left(-\nabla_{\theta} \cdot b-i b \cdot \nabla_{\theta} \varphi-\nabla_{x} \cdot c-i c \cdot \nabla_{x} \varphi+\nabla_{\theta} \cdot b+\nabla_{x} \cdot c+\phi^{0}\right) e^{i \varphi} \\
& =\left(\left(1-\phi^{0}\right) \cdot \Phi^{-1} \cdot \Phi+\phi^{0}\right) e^{i \varphi}=e^{i \varphi},
\end{aligned}
$$

where we have made use of the definition 3.6 of the symbol $\Phi$.

The continuity (3.12) of $P$ is immediate, by the continuity of differentiation and multiplication by another symbol as operations between SG-classes, see Section 1.2.3, in particular the orders in 3.9 match in exactly such a way that the continuity between the indicated spaces follows.

The next lemma allows us to estimate (3.2) with respect to the topologies of $\mathscr{S}\left(\mathbb{R}^{d} \times \mathbb{R}^{s}\right)$ and $\mathrm{SG}^{m_{e}, m_{\psi}}\left(\mathbb{R}^{d} \times \mathbb{R}^{s}\right)$. It essentially coincides with [49, Lem. 5].

Lemma 3.11. Let $\varphi$ be an admissible inhomogeneous SG-phase function of order $\left(n_{e}, n_{\psi}\right)$ and $a \in \mathscr{S}\left(\mathbb{R}^{d} \times \mathbb{R}^{s}\right)$. Then, the associated oscillatory integral $I_{\varphi}(a)$, 
defined in (3.1), is an element of $\mathscr{S}\left(\mathbb{R}^{d}\right)$ that satisfies, when considered as a (regular) tempered distribution, for any $\left(m_{e}, m_{\psi}\right) \in \mathbb{R}^{2}$ and each $f \in \mathscr{S}\left(\mathbb{R}^{d}\right)$,

$$
\left|\left\langle I_{\varphi}(a), f\right\rangle\right| \lesssim \rho_{M}^{m_{e}, m_{\psi}}(a) \rho_{N}^{\mathscr{S}}(f),
$$

where the indices $N$ and $M$ depend solely on $\left(m_{e}, m_{\psi}\right)$.

Proof. The fact that (3.1) converges for every $x \in \mathbb{R}^{d}$ and yields a smooth and rapidly decreasing function in $x$ follows from

$$
\left|\int_{\mathbb{R}^{s}} e^{i \varphi(x, \theta)} a(x, \theta) d \theta\right| \leq \int_{\mathbb{R}^{s}}|a(x, \theta)| d \theta,
$$

the rapid decay of $a$ in both $x$ and $\theta$, differentiation under the integral sign and dominated convergence.

By applying the operator defined in Lemma 3.10 several times, $r \in \mathbb{N}_{0}$, we obtain, for a fixed $f \in \mathscr{S}\left(\mathbb{R}^{d}\right)$,

$$
\begin{aligned}
\left|\int_{\mathbb{R}^{s} \times \mathbb{R}^{d}} e^{i \varphi(x, \theta)} a(x, \theta) f(x) d \theta d x\right| & =\left|\int_{\mathbb{R}^{s} \times \mathbb{R}^{d}}\left({ }^{t} P\right)^{r} e^{i \varphi(x, \theta)} a(x, \theta) f(x) d \theta d x\right| \\
& \leq \int_{\mathbb{R}^{s} \times \mathbb{R}^{d}}\left|P^{r}(a(x, \theta) f(x))\right| d \theta d x .
\end{aligned}
$$

Since multiplication by $f \in \mathscr{S}\left(\mathbb{R}^{d}\right)$ (in the first set of variables) is a continuous map $\mathrm{SG}^{m_{e}, m_{\psi}} \rightarrow \mathrm{SG}^{-\infty, m_{\psi}}$ and the inclusion map $\mathrm{SG}^{m_{e}^{\prime}, m_{\psi}^{\prime}} \hookrightarrow \mathrm{SG}^{m_{e}, m_{\psi}}, m_{e}^{\prime} \leq m_{e}$, $m_{\psi}^{\prime} \leq m_{\psi}$, is continuous, we have that the map given by $a \mapsto P^{r}(a(x, \theta) f(x))$ is a continuous map from $\mathrm{SG}^{m_{e}, m_{\psi}}\left(\mathbb{R}^{d} \times \mathbb{R}^{s}\right)$ to $\mathrm{SG}^{m_{e}-r n_{e}, m_{\psi}-r n_{\psi}}\left(\mathbb{R}^{d} \times \mathbb{R}^{s}\right)$ for any $r \in \mathbb{N}_{0}$. In particular we have

$$
\sup _{\mathbb{R}^{d} \times \mathbb{R}^{s}}\left|P^{r}(a(x, \theta) f(x))\right|\langle x\rangle^{r n_{e}-m_{e}}\langle\theta\rangle^{r n_{\psi}-m_{\psi}} \lesssim \rho_{M}^{m_{e}, m_{\psi}}(a) \rho_{N}^{\mathscr{S}}(f)
$$

with $M$ and $N$ in $\mathbb{N}_{0}$ depending solely on $r$ and the orders $\left(m_{e}, m_{\psi}\right)$ and $\left(n_{e}, n_{\psi}\right)$. Thus, for suitably large $r$ (such that the following integral converges) we have

$\left|\left\langle I_{\varphi}(a), f\right\rangle\right| \lesssim \rho_{M}^{m_{e}, m_{\psi}}(a) \rho_{N}^{\mathscr{S}}(f) \int_{\mathbb{R}^{s} \times \mathbb{R}^{d}}\langle x\rangle^{m_{e}-r n_{e}}\langle\theta\rangle^{m_{\psi}-r n_{\psi}} d \theta d x \lesssim \rho_{M}^{m_{e}, m_{\psi}}(a) \rho_{N}^{\mathscr{S}}(f)$ which proves the claim.

Using the previous lemmas, we may conclude the proof of Theorem 3.9 as in [49]. 
Proof of Theorem 3.9. Looking at the proof of Lemma 3.11, it is possible to define, for $f \in \mathscr{S}\left(\mathbb{R}^{d}\right)$ and $r \in \mathbb{N}_{0}$ large enough (which depends, for a fixed admissible phase-function $\varphi$, only on the order $\left(m_{e}, m_{\psi}\right)$ of $\left.a\right)$,

$$
\left\langle I_{\varphi}(a), f\right\rangle:=\int_{\mathbb{R}^{s} \times \mathbb{R}^{d}} e^{i \varphi(x, \theta)} P^{r}(a(x, \theta) f(x)) d \theta d x .
$$

That this is a well-defined tempered distribution is granted by (3.13). By the continuity with respect to the symbol topologies we may conclude that this is indeed a well-defined (independently of $r$, provided it is chosen large enough) and unique continuation of the map defined by $(3.2)$, since we may approximate any amplitude by a series of Schwartz functions, as granted by Lemma A.11.

Remark 3.12. In a similar approach of defining oscillatory integrals, one makes use of a mollifier $\phi_{\epsilon}^{0}$, setting

$$
\left\langle I_{\varphi}(a), f\right\rangle:=\lim _{\epsilon \rightarrow 0}\left\langle e^{i \varphi}, a \cdot\left(f \otimes \phi_{\epsilon}^{0}\right)\right\rangle .
$$

Due to the uniqueness statement in Theorem 3.9, the two methods coincide 45 Both approaches underline how the definition of $I_{\varphi}(a)$ can actually be seen as the extension of the distribution $e^{i \varphi}$ from the space

$\underbrace{\mathrm{SG}^{-\infty, 0}\left(\mathbb{R}^{d} \times \mathbb{R}^{s}\right)}_{\ni f \otimes 1} \cdot \underbrace{\mathrm{SG}^{m_{e},-\infty}\left(\mathbb{R}^{d} \times \mathbb{R}^{d}\right)}_{\ni \phi_{\epsilon} a}=\mathscr{S}\left(\mathbb{R}^{d}\right)\left(\mathbb{R}^{d} \times \mathbb{R}^{d}\right) \rightarrow \underbrace{\mathrm{SG}^{-\infty, m_{\psi}}\left(\mathbb{R}^{d} \times \mathbb{R}^{s}\right)}_{\ni f \cdot a}$.

This is useful to keep in mind during Section 3.3.2, where we will consider extensions of tempered distributions.

\subsubsection{Singularities of tempered oscillatory integrals.}

Having defined oscillatory integrals, we may turn to a description of their singularities, essentially following our approach in [49, Sect. 4]. In order to do so, we first generalize the notion of $\mathcal{C}_{\varphi}$ as encountered in Section 3.2.1. In the following, we again make systematic use of the identification $\mathbb{R}^{d} \sqcup\left(\left(\mathbb{R}^{d} \backslash\{0\}\right) / \sim\right) \cong \mathbb{B}^{d}$ of Construction 1.1 in terms of the isomorphism $\iota$ and the identification $\partial \mathbb{B}^{d}=$ $\partial \mathbb{S}^{d-1}=\left(\mathbb{R}^{d} \backslash\{0\}\right) / \sim$, as well as of the conification map $\Gamma$. Again, we consider subsets of $\left(\mathbb{R}^{d} \backslash\{0\}\right) / \sim$ as conic subsets of $\mathbb{R}^{d} \backslash\{0\}$.

In the upcoming constructions, we will often pass from one of these viewpoints to the other: whenever we make use of the conic structure, we work on $\mathbb{R}^{d} \sqcup\left(\mathbb{R}^{d} \backslash\{0\}\right)$

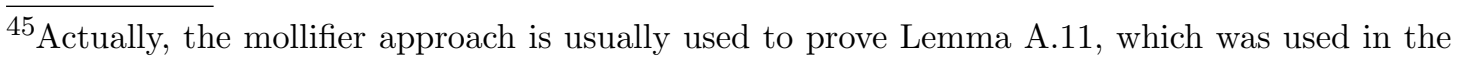
proof. 
and when we analyse "topological properties at infinity", we work on $\mathbb{B}^{d}$. In order to facilitate the comprehension, we adopt the notation of [50], which is introduced in the following.

\section{Notation.}

- $y$ denotes "variable-type" elements of $\mathbb{B}^{d}, \eta$ denotes "co-variable-type" elements of $\mathbb{B}^{d}, \gamma$ denotes "co-variable-type" elements of $\mathbb{B}^{s}$,

- the corresponding elements of $\mathbb{R}^{d} \sqcup\left(\mathbb{R}^{d} \backslash\{0\}\right)$ are denoted by $x$ and $\xi$ respectively and elements of $\mathbb{R}^{s} \sqcup\left(\mathbb{R}^{s} \backslash\{0\}\right)$ are named $\theta$.

In a completely similar fashion to the definition of $\widetilde{\mathrm{W}}_{\mathrm{SG}}=\partial\left(\mathbb{B}^{d} \times \mathbb{B}^{d}\right)$, substituting $s$ in place of $d$ in the dimensions of the second factors in $(1.2)$, we define $\widetilde{\mathrm{B}}:=$ $\partial\left(\mathbb{B}^{d} \times \mathbb{B}^{s}\right)=\widetilde{\mathrm{B}}^{\psi} \cup \widetilde{\mathrm{B}}^{e} \cup \widetilde{\mathrm{B}}^{\psi e}$ and, again with $s$ in place of $d$ in the dimensions of the second factors of $(1.3), \mathrm{B}:=\mathrm{B}^{\psi} \sqcup \mathrm{B}^{e} \sqcup \mathrm{B}^{\psi e}$. Finally, we set $\mathrm{S}=\mathrm{S}^{\psi} \sqcup \mathrm{S}^{e} \sqcup \mathrm{S}^{\psi e}$, with

$$
\mathrm{S}^{\psi}=\mathbb{R}^{d} \times \mathbb{S}^{s-1} \quad \mathrm{~S}^{e}=\mathbb{S}^{d-1} \times \mathbb{R}^{s} \quad \mathrm{~S}^{\psi e}=\mathbb{S}^{d-1} \times \mathbb{S}^{s-1}
$$

Remark 3.13. Note that we may also formulate for instance

$$
\begin{aligned}
\mathrm{B}^{\psi} & =(\mathrm{id} \times \Gamma)\left(\mathrm{S}^{\psi}\right)=\left(\iota^{-1} \times \Gamma\right)\left(\widetilde{\mathrm{B}}^{\psi}\right) \\
\mathrm{B}^{e} & =(\Gamma \times \mathrm{id})\left(\mathrm{S}^{e}\right)=\left(\Gamma \times \iota^{-1}\right)\left(\widetilde{\mathrm{B}}^{e}\right) \\
\mathrm{B}^{\psi e} & =(\Gamma \times \Gamma)\left(\mathrm{S}^{\psi e}\right)=(\Gamma \times \Gamma)\left(\widetilde{\mathrm{B}}^{\psi e}\right)
\end{aligned}
$$

Definition 3.14. Let $\varphi \in \mathrm{SG}^{n_{e}, n_{\psi}}\left(\mathbb{R}^{d} \times \mathbb{R}^{s}\right)$ an admissible phase function. Then $\langle\theta\rangle^{2}\left|\nabla_{\theta} \varphi\right|^{2} \in \mathrm{SG}^{2\left(n_{e}, n_{\psi}\right)}\left(\mathbb{R}^{d} \times \mathbb{R}^{s}\right)$. Then $\widetilde{\mathcal{C}}_{\varphi}$ denotes the (closed) set ${ }^{46}$

$$
\widetilde{\mathcal{C}}_{\varphi}:=\left\{\left.\left(y_{0}, \gamma_{0}\right) \in \partial\left(\mathbb{B}^{d} \times \mathbb{B}^{s}\right)\left|\langle\theta\rangle^{2}\right| \nabla_{\theta} \varphi\right|^{2} \text { is not elliptic at }\left(y_{0}, \gamma_{0}\right)\right\}
$$

We now establish the first half of an analogue to Theorem 3.2 .

Proposition 3.15. Let $\varphi$ an admissible phase function. Then for any amplitude $a \in \mathrm{SG}\left(\mathbb{R}^{d} \times \mathbb{R}^{s}\right)$ we have

$$
\widetilde{\operatorname{Css}}\left(I_{\varphi}(a)\right) \subset \operatorname{pr}_{1}\left(\widetilde{\mathcal{C}_{\varphi}}\right) .
$$

As in [49], we conclude this Proposition as a consequence of the following one:

\footnotetext{
${ }^{46}$ I.e. we have $\widetilde{\mathcal{C}}_{\varphi}=\widetilde{\operatorname{char} S G}\left(\left|\nabla_{\theta} \varphi\right|^{2}\right)$.
} 
Proposition 3.16. Let $\varphi$ be an admissible inhomogeneous SG-phase function of order $\left(n_{e}, n_{\psi}\right)$ and let $a \in \mathrm{SG}\left(\mathbb{R}^{d} \times \mathbb{R}^{s}\right)$.

If $\widetilde{\operatorname{Csp}}(a) \cap \widetilde{\mathcal{C}_{\varphi}}=\emptyset$, then $\widetilde{\operatorname{Css}}\left(I_{\varphi}(a)\right)=\emptyset$, that is, $I_{\varphi}(a) \in \mathscr{S}\left(\mathbb{R}^{d}\right)$.

We delay the proof of this statement to first conclude Proposition 3.15.

Proof of Proposition 3.15. Let $y_{0} \notin \operatorname{pr}_{1}\left(\widetilde{\mathcal{C}}_{\varphi}\right)$. Choose a (possibly asymptotic) cutoff $\phi^{x_{0}}$ around the element $x_{0}$ corresponding to $y_{0}$ such that $\widetilde{\operatorname{Csp}}\left(\phi^{x_{0}}\right) \cap \operatorname{pr}_{1}\left(\widetilde{\mathcal{C}}_{\varphi}\right)=\emptyset$. Revisiting the construction of the map $I_{\varphi}$ in the proof of Theorem 3.9, we have $\phi^{x_{0}} I_{\varphi}(a)=I_{\varphi}\left(\phi^{x_{0}} a\right)$, and the latter belongs to $\mathscr{S}\left(\mathbb{R}^{d}\right)$, by Proposition 3.16 .

Proof of Proposition 3.16. We follow our proof in [49], which is again based on a regularization argument, cf. [156, Prop. 3.3] and the original [79, Prop. 1.2.5.].

Choose first a neighbourhood $\widetilde{V} \subset \mathbb{B}^{d} \times \mathbb{B}^{s}$ of $\widetilde{\operatorname{Csp}}(a)$ whose closure does not intersect $\widetilde{\mathcal{C}}_{\varphi}$. Then, by the definition of $\widetilde{\mathcal{C}}_{\varphi},\left|\nabla_{\theta} \varphi(x, \theta)\right|^{2}$ is elliptic at each $\left(y_{0}, \gamma_{0}\right)$ in $\widetilde{V} \cap \widetilde{\mathrm{B}}$. By compactness, we can then obtain a single open neighbourhood $\widetilde{U} \subset \mathbb{B}^{d} \times \mathbb{B}^{s}$ of $\operatorname{Csp}(a) \cap \widetilde{\mathrm{B}} \subset\left(\mathbb{B}^{d} \times \mathbb{B}^{s}\right)$ with $\widetilde{U} \subset \widetilde{V}$ such that

$$
\left|\nabla_{\theta} \varphi(x, \theta)\right|^{2} \gtrsim\langle x\rangle^{2 n_{e}}\langle\theta\rangle^{2 n_{\psi}-2} \quad \forall(x, \theta) \in\left(\iota^{-1} \times \iota^{-1}\right)\left(\widetilde{U}^{o}\right) .
$$

We then fix a cut-off function $\phi_{U}$, as in Construction 1.3 (see Figure 2) identically equal to $\mathbb{1}$ on $\iota\left(\widetilde{U}^{o}\right)$ with $\widetilde{\operatorname{Csp}}\left(\phi_{U}\right) \subset \widetilde{V}$ such that we have the estimate 3.15 on all of the support of $\phi_{U}$. We now write $I_{\varphi}(a)=I_{\varphi}\left(\phi_{U} a\right)+I_{\varphi}\left(\left(\mathbb{1}-\phi_{U}\right) a\right)$. By construction, $\left(\mathbb{1}-\phi_{U}\right) a$ is compactly supported, which implies $I_{\varphi}\left(\left(\mathbb{1}-\phi_{U}\right) a\right) \in$ $\mathscr{S}\left(\mathbb{R}^{d}\right)$. To analyse $I_{\varphi}\left(\phi_{U} a\right)$, we define

$$
\begin{gathered}
b_{j}(x, \theta)=i\left|\nabla_{\theta} \varphi(x, \theta)\right|^{-2} \partial_{\theta_{j}} \varphi(x, \theta), \quad c(x, \theta)=\nabla_{\theta} \cdot b(x, \theta), \\
Q=b \cdot \nabla_{\theta}+c .
\end{gathered}
$$

Observe that $b_{j}$ is well-defined on $\operatorname{supp}\left(\phi_{U}\right)$, since $\left|\nabla_{\theta} \varphi\right|^{2}$ is strictly positive on $\operatorname{supp}\left(\phi_{U}\right)$, and as a consequence of 3.15 we have that

$$
\phi_{U} b_{j} \in \mathrm{SG}^{-n_{e},-n_{\psi}+1}\left(\mathbb{R}^{d} \times \mathbb{R}^{s}\right) \quad \text { and } \quad \phi_{U} c \in \mathrm{SG}^{-n_{e},-n_{\psi}}\left(\mathbb{R}^{d} \times \mathbb{R}^{s}\right) .
$$

Furthermore we may compute

$$
\phi_{U}\left[{ }^{t} Q e^{i \varphi}\right]=\phi_{U}\left(-\nabla_{\theta} \cdot b-i b \cdot \nabla_{\theta} \varphi+\nabla_{\theta} \cdot b\right) e^{i \varphi}=\phi_{U} e^{i \varphi} .
$$

The same holds true if we replace $\phi_{U}$ by any SG-symbol of order $(0,0)$ with the same support properties. We can then conclude by an approximation argument as 
in the proof of Theorem 3.9 and in the proof of Lemma 3.11:

$Q$ involves only differentiations with respect to $\theta$ and as a consequence we can insert it into the expression of $I_{\varphi}\left(\phi_{U} a\right)$, and find, using (3.16),

$$
I_{\varphi}\left(\phi_{U} a\right)=I_{\varphi}\left(Q^{r}\left(\phi_{U} a\right)\right) \text { for arbitrary } r \in \mathbb{N}_{0} .
$$

since the identity holds true for $a \in \mathscr{S}\left(\mathbb{R}^{d} \times \mathbb{R}^{s}\right)$ supported in $V$ and extends by continuity.

Since $Q$ is a continuous map from $\mathrm{SG}^{m_{e}, m_{\psi}}\left(\mathbb{R}^{d} \times \mathbb{R}^{s}\right)$ to $\mathrm{SG}^{m_{e}-n_{e}, m_{\psi}-n_{\psi}}\left(\mathbb{R}^{d} \times \mathbb{R}^{s}\right)$, we can achieve arbitrarily low order of $Q^{r}\left(\phi_{U} a\right)$, by choosing $r$ large enough. Thus $I_{\varphi}\left(\phi_{U} a\right) \in \mathscr{S}\left(\mathbb{R}^{d}\right)$, and consequently $I_{\varphi}(a)=I_{\varphi}\left(\phi_{U} a\right)+I_{\varphi}\left(\left(\mathbb{1}-\phi_{U}\right) a\right) \in \mathscr{S}\left(\mathbb{R}^{d}\right)$, which proves the claim.

Having suitably generalized $\mathcal{C}_{\varphi}$, we can turn to generalizing $\Lambda_{\varphi}$ as in [49]. The following definition may seem, at first, a bit tedious to compute for general phase functions, but it will simplify a great deal and obtain a clear geometric interpretation if we consider a SG-phase function $\varphi \in \mathrm{SG}_{\mathrm{cl}}^{1,1}$, which we will do in Section 3.2 .4

Definition 3.17. Let $\varphi$ a SG-phase function $\varphi$ of order $\left(n_{e}, n_{\psi}\right)$. Denote by $\operatorname{pr}_{\widetilde{\mathcal{C}}_{\varphi}}$ the projection of $\widetilde{\mathcal{C}}_{\varphi} \times \mathbb{B}^{d} \subset \partial\left(\mathbb{B}^{d} \times \mathbb{B}^{s}\right) \times \mathbb{B}^{d}$ onto $\mathbb{B}^{d} \times \mathbb{B}^{d}$. We define the set of stationary phase points of $\varphi, \widetilde{\Lambda}_{\varphi} \subset \widetilde{\mathrm{W}}_{\mathrm{SG}}$, given in terms of its complement (with respect to $\left.\widetilde{\mathrm{W}}_{\mathrm{SG}}\right)$ by

$$
\begin{aligned}
\left(\tilde{\Lambda}_{\varphi}\right)^{c}:=\left\{\left(y_{0}, \eta_{0}\right) \in \widetilde{\mathrm{W}}_{\mathrm{SG}}:\right. & \exists \widetilde{U} \text { open neighbourhood of }\left(y_{0}, \eta_{0}\right) \text { in } \mathbb{B}^{d} \times \mathbb{B}^{d} \\
& \exists \widetilde{V} \text { open neighbourhood of } \operatorname{pr}_{\widetilde{\mathcal{C}}_{\varphi}}^{-1}(\widetilde{U}) \text { such that } \\
& \left|\nabla_{x} \varphi(x, \theta)-\xi\right| \gtrsim\langle x\rangle^{n_{e}-1}\langle\theta\rangle^{n_{\psi}}+|\xi| \\
& \text { for any } \left.(x, \theta, \xi) \in\left(\iota^{-1} \times \iota^{-1} \times \iota^{-1}\right)\left(\widetilde{V}^{o}\right)\right\} .
\end{aligned}
$$

As usual, we set

$$
\tilde{\Lambda}_{\varphi}^{e}:=\tilde{\Lambda}_{\varphi} \cap \widetilde{\mathrm{W}}_{\mathrm{SG}}^{e}, \quad \tilde{\Lambda}_{\varphi}^{\psi}:=\tilde{\Lambda}_{\varphi} \cap \widetilde{\mathrm{W}}_{\mathrm{SG}}^{\psi}, \quad \tilde{\Lambda}_{\varphi}^{\psi e}:=\tilde{\Lambda}_{\varphi} \cap \widetilde{\mathrm{W}}_{\mathrm{SG}}^{\psi e} .
$$

$\tilde{\Lambda}_{\varphi}$ is defined as the complement of a manifestly open set in $\widetilde{W}_{\mathrm{SG}}$ and therefore constitutes a closed subset of $\widetilde{\mathrm{W}}_{\mathrm{SG}}$ and consequently also of $\mathbb{B}^{d} \times \mathbb{B}^{d}$. We are now ready to prove an analogue of Theorem 3.2 by following our proof of 49, Thm. 4.1], which is again inspired by [156] and the classical [79, Prop. 2.5.7] by applying, 
as in the previous proofs, integral regularization to the expressions that arise when determining $\widetilde{\mathrm{WF}}_{\mathrm{SG}}\left(I_{\varphi}(a)\right.$.

Theorem 3.18. Let $\varphi$ be an admissible inhomogeneous SG-phase function of order $\left(n_{e}, n_{\psi}\right)$ and let $a \in \mathrm{SG}\left(\mathbb{R}^{d} \times \mathbb{R}^{s}\right)$. For the temperate oscillatory integral $I_{\varphi}(a)$, defined in Theorem 3.9, we have

$$
\widetilde{\mathrm{WF}}_{\mathrm{SG}}\left(I_{\varphi}(a)\right) \subset \tilde{\Lambda}_{\varphi}
$$

Proof. Let $\left(y_{0}, \eta_{0}\right) \in \widetilde{\mathrm{W}}_{\mathrm{SG}} \backslash \tilde{\Lambda}_{\varphi}$ and denote by $\left(x_{0}, \xi_{0}\right)$ the corresponding point in $\mathrm{W}_{\mathrm{SG}}$. By Prop. 3.15, we may limit our attention $y_{0} \in \operatorname{pr}_{1}\left(\widetilde{\mathcal{C}}_{\varphi}\right)$, since otherwise $y_{0} \notin \operatorname{pr}_{1}\left(\widetilde{\mathcal{C}}_{\varphi}\right) \supset \widetilde{\operatorname{Css}}\left(I_{\varphi}(a)\right)=\operatorname{pr}_{1}\left(\widetilde{\mathrm{WF}}_{\mathrm{SG}}\left(I_{\varphi}(a)\right)\right)$.

Using the characterization of $\widetilde{W F}_{\mathrm{SG}}$ in terms of cut-offs, i.e. Lemma 2.5, we have to prove that there exists a pair of (asymptotic) cut-offs $\phi^{x_{0}}$ and $\phi^{\xi_{0}}$ such that on the support of $\phi^{\xi_{0}}$ we have

$$
\phi^{\xi_{0}} \mathcal{F}_{x \rightarrow \xi}\left[\phi^{x_{0}} I_{\varphi}(a)\right] \in \mathscr{S}\left(\mathbb{R}^{d}\right) .
$$

In fact, we will show that for $a \in \mathscr{S}\left(\mathbb{R}^{d}\right)$

$$
\left|\phi^{\xi_{0}} \mathcal{F}\left[\phi^{x_{0}} I_{\varphi}(a)\right](\xi)\right| \lesssim \rho_{M}^{m_{e}, m_{\psi}}(a)(1+|\xi|)^{-N},
$$

for arbitrarily high $N \in \mathbb{N}_{0}$, some $M \in \mathbb{N}_{0}$ and any $\xi \in \operatorname{supp}\left(\phi^{\xi_{0}}\right)$. Then an approximation argument as in the proof of Theorem 3.9 yields the result.

Since $\left(y_{0}, \eta_{0}\right) \notin \tilde{\Lambda}_{\varphi}$ we can, by Definition 3.17 , find an open neighbourhood $\widetilde{U}$ of $\left(y_{0}, \eta_{0}\right)$ and a triple of localizing cut-offs $\left(\phi^{x_{0}}(x), \phi^{\xi_{0}}(\xi), \phi(\theta)\right)$ such that

- the product $\psi^{x_{0}}(x) \phi(\theta) \phi^{\xi_{0}}(\xi)$ does not vanish on some neighbourhood of $\left(\iota^{-1} \times \iota^{-1} \times \iota^{-1}\right)\left(\operatorname{pr}_{\widetilde{\mathcal{C}}_{\varphi}}^{-1}(\widetilde{U})\right)^{o}$

- on the support of $\psi^{x_{0}}(x) \phi(\theta) \phi^{\xi_{0}}(\xi)$ the function defined by $\Psi(x, \theta, \xi):=$ $\left|\nabla_{x} \varphi(x, \theta)-\xi\right|^{2}$ fulfils

$$
\Psi(x, \theta, \xi) \gtrsim\left(\langle x\rangle^{n_{e}-1}\langle\theta\rangle^{n_{\psi}}+|\xi|\right)^{2} .
$$

The supports of the cut-offs were chosen in such a way that $\widetilde{\operatorname{Csp}}\left(\phi^{x_{0}} \otimes[1-\phi]\right)$ does not intersect $\widetilde{\mathcal{C}}_{\varphi}$. Thus, by Proposition 3.15. we can restrict our analysis to an amplitude of the form $\phi(\theta) a(x, \theta)$ by writing

$$
\phi^{x_{0}} I_{\varphi}(a)=\underbrace{I_{\varphi}\left(\left(\phi^{x_{0}} \otimes(\mathbb{1}-\phi)\right) a\right)}_{\in \mathscr{S}\left(\mathbb{R}^{d}\right)}+I_{\varphi}\left(\left(\phi^{x_{0}} \otimes \phi\right) a\right) .
$$


In the remainder of the proof we thus assume $a$ to be supported in such a way that on the support of $\phi^{\xi_{0}}(\xi) \phi^{x_{0}}(x) a(x, \theta)$ we can estimate $\tilde{\Phi}$ as in $(3.20)$. For now assume further $a \in \mathscr{S}\left(\mathbb{R}^{d} \times \mathbb{R}^{s}\right)$.

We define another operator for the purpose of integral regularization via

$$
\begin{aligned}
b_{j}(x, \theta, \xi) & =i \Psi(x, \theta, \xi)^{-1} \phi^{x_{0}}(x)\left(\partial_{x_{j}} \varphi(x, \theta)-\xi_{j}\right), \\
c(x, \theta, \xi) & =\nabla_{x} \cdot b(x, \theta, \xi), \\
Q & =b \cdot \nabla_{x}+c .
\end{aligned}
$$

By the previous assumptions on the supports, the operator $Q$ is well-defined on the support of $\phi^{\xi_{0}}(\xi) a(x, \xi)$. We construct the transpose of $Q$ (boundary terms vanish since $\left.a \in \mathscr{S}\left(\mathbb{R}^{d} \times \mathbb{R}^{s}\right)\right)$ and conclude that on the support of $\phi^{\xi_{0}} a$ we have

$$
\begin{aligned}
{ }^{t} Q & e^{i \varphi(x, \theta)-i x \cdot \xi}= \\
& =\left[-\nabla_{x} \cdot b(x, \theta, \xi)-i b(x, \theta, \xi) \cdot\left(\nabla_{x} \varphi(x, \theta)-\xi\right)+\nabla_{x} \cdot b(x, \xi, p)\right] e^{i \varphi(x, \theta)-i x \cdot \xi} \\
& =\phi^{x_{0}} e^{i \varphi(x, \theta)-i x \cdot \xi} .
\end{aligned}
$$

Since we may assume $\phi^{x_{0}} \equiv 1$ in a neighbourhood of $x_{0}$, we can pick another (asymptotic) cut-off $\phi_{2}^{x_{0}}$ supported in that smaller neighbourhood of $x_{0}$ such that $\phi_{2}^{x_{0}} \phi^{x_{0}}=\phi_{2}^{x_{0}}$.

By (3.21), for arbitrary $r \in \mathbb{N}_{0}$, we have

$$
\begin{aligned}
\left|\phi^{\xi_{0}} \mathcal{F}\left[\phi_{2}^{x_{0}} I_{\varphi}(a)\right](\xi)\right| & =\left|\phi^{\xi_{0}}(\xi) \int_{\mathbb{R}^{d} \times \mathbb{R}^{s}} e^{i \varphi(x, \theta)-i x \cdot \xi} \phi_{2}^{x_{0}}(x) a(x, \theta) d x d \theta\right| \\
& \leq\left|\int_{\mathbb{R}^{d} \times \mathbb{R}^{s}} e^{i \varphi(x, \theta)-i x \cdot p} Q^{r}\left(\phi^{\xi_{0}}(\xi) \phi_{2}^{x_{0}}(x) a(x, \theta)\right) d x d \theta\right| \\
& \leq \int_{\mathbb{R}^{d} \times \mathbb{R}^{s}}\left|Q^{r}\left(\phi^{\xi_{0}}(\xi) \phi_{2}^{x_{0}}(x) a(x, \theta)\right)\right| d x d \theta .
\end{aligned}
$$

By (3.20), using the fact that differentiation decreases the respective symbol order by 1 , for any $\left(m_{e}, m_{\psi}\right)$ there exists a semi-norm $\rho_{M}^{m_{e}, m_{\psi}}$ on $\mathrm{SG}^{m_{e}, m_{\psi}}\left(\mathbb{R}^{d} \times \mathbb{R}^{s}\right)$ such that

$$
\left|Q^{r}\left(\phi^{\xi_{0}}(\xi) \phi_{2}^{x_{0}}(x) a(x, \theta)\right)\right| \lesssim \rho_{M}^{m_{e}, m_{\psi}}(a)\langle x\rangle^{m_{e}-r n_{e} / 2}\langle\theta\rangle^{m_{\psi}-r n_{\psi} / 2}(1+|\xi|)^{-r / 2} .
$$

Therefore, for large enough $r$, the final integral in 3.22 is integrable and decays in $\xi$ faster than any inverse power, we have therefore proved (3.19). By differentiating under the integral sign, we can show similar estimates for any derivative with 
respect to $\xi$. If we approximate general $a \in \mathrm{SG}^{m_{e}, m_{\psi}}\left(\mathbb{R}^{d} \times \mathbb{R}^{s}\right)$ via test functions 4 this proves the claim.

\subsubsection{A study of the underlying geometry.}

In the previous setting, we have defined oscillatory integrals for a very general class of phase functions and amplitudes. In the following, we will restrict our attention to classical phases (in the SG-sense of classicality) of order $(1,1)$, meaning $\varphi \in \mathrm{SG}_{\mathrm{cl}}^{1,1}\left(\mathbb{R}^{d} \times \mathbb{R}^{s}\right)$.

Under this stronger assumption, we will, following [50], calculate the objects $\widetilde{\mathcal{C}}_{\varphi}$ and $\tilde{\Lambda}_{\varphi}$ in terms of the principal symbol of $\varphi$ and discuss their geometric properties. The approach will be to use Proposition 1.20 and Construction 1.1 to associate to $\widetilde{\mathcal{C}}_{\varphi}$ and $\tilde{\Lambda}_{\varphi}$ triples of conic submanifolds, much in the same way as one associates to a classical symbol a triple of homogeneous principal symbols. Consequently, we first discuss the principal symbol of a $\mathrm{SG}_{\mathrm{cl}}^{1,1}$-phase function.

By (1.11), we can write (using excision functions $\chi^{e}$ and $\chi^{\psi}$ ) and the notation $\bullet \in\{\psi, e, \psi e\}$ and $\sigma \bullet(\phi)=: \phi^{\bullet}$

$$
\varphi(x, \theta)=\chi^{e} \varphi^{e}+\chi^{\psi} \varphi^{\psi}-\chi^{e} \chi^{\psi} \varphi^{\psi e}+r_{\varphi}
$$

with the principal symbol of $\sigma(\varphi)=\left(\varphi^{e}, \varphi^{\psi}, \varphi^{\psi e}\right)$ where

- $\varphi^{e} \in \mathscr{P}_{e}^{1,1}\left(\mathbb{R}^{d} \times \mathbb{R}^{s}\right)$ is $\psi$-polyhomogeneous,

- $\varphi^{\psi} \in \mathscr{P}_{\psi}^{1,1}\left(\mathbb{R}^{d} \times \mathbb{R}^{s}\right)$ is e-polyhomogeneous,

- $\varphi^{\psi e} \in \mathscr{P}_{\psi e}^{1,1}\left(\mathbb{R}^{d} \times \mathbb{R}^{s}\right)$ satisfies $\sigma_{\psi}\left(\varphi^{e}\right)=\sigma_{e}\left(\varphi^{\psi}\right)=\varphi^{\psi e}$,

- $r_{\varphi} \in \mathrm{SG}^{0,0}\left(\mathbb{R}^{d} \times \mathbb{R}^{s}\right)$.

As in Remark 3.8 we may absorb $e^{i r_{\varphi}} \in \mathrm{SG}^{0,0}\left(\mathbb{R}^{d} \times \mathbb{R}^{s}\right)$ into the amplitude of an oscillatory integral. We have thus reduced our study to the case of phase functions of the form

$$
\varphi(x, \theta)=\chi^{e}(x) \varphi^{e}(x, \theta)+\chi^{\psi}(\theta) \varphi^{\psi}(x, \theta)-\chi^{e}(x) \chi^{\psi}(\theta) \varphi^{\psi e}(x, \theta) .
$$

In order to attach geometric meaning to the singularity structures, we work on the compactification $\widetilde{B}$. We must first find a suitable representation of the phase function over that space. We recall that, by Proposition 1.20 , we have

$$
\tilde{\varphi}:=\left(\iota^{-1} \times \iota^{-1}\right)^{*} \varphi \in \tilde{y}^{-1} \tilde{\gamma}^{-1} \mathscr{C}^{\infty}\left(\mathbb{B}^{d} \times \mathbb{B}^{s}\right) .
$$

\footnotetext{
$\overline{47}$ This approximation has to be compatible with the support assumptions on $a$. Reviewing the proof of [113, Prop. 1.1.5]), that is of Lemma A.11, this can be achieved.
} 
This allows us to work with smooth functions (multiplied by a weight function) instead of symbols. However, we remark that one has to be careful when differentials are involved, since we have

$$
\begin{aligned}
& \tilde{\gamma} \widetilde{\nabla_{x} \varphi}:=\left(\iota_{\mathrm{SG}}^{0,1}\left(\nabla_{x} \varphi\right)\right)(y, \gamma)=\tilde{\gamma} \nabla_{y} \tilde{\varphi}(y, \gamma) \cdot\left(\frac{d \iota^{-1}(y)}{d y}\right)^{-1} . \\
& \tilde{y} \widetilde{\nabla_{\theta} \varphi}:=\left(\iota_{\mathrm{SG}}^{1,0}\left(\nabla_{\theta} \varphi\right)\right)(y, \gamma)=\tilde{y} \nabla_{\gamma} \tilde{\varphi}(y, \gamma) \cdot\left(\frac{d \iota^{-1}(\gamma)}{d \gamma}\right)^{-1} .
\end{aligned}
$$

As in [50], we now analyse how the additional structure which we put on $\varphi$ translates to the object $\tilde{\varphi}$ and how we are able to characterize $\widetilde{\mathcal{C}}_{\varphi}$ in terms of $\tilde{\varphi}$.

Lemma 3.19. The ellipticity assumption (3.7) on the associated function

$$
\Phi=\left|\langle x\rangle \nabla_{x} \varphi\right|^{2}+\left|\langle\theta\rangle \nabla_{\theta} \varphi\right|^{2}
$$

is equivalent to the condition that $\left(\tilde{\gamma} \widetilde{\nabla_{x} \varphi}, \widetilde{y} \widetilde{\nabla_{\theta} \varphi}\right)$ is nowhere vanishing on $\widetilde{\mathrm{B}}$. Furthermore, we can write

$$
\widetilde{\mathcal{C}}_{\varphi}=\left\{\left(y_{0}, \gamma_{0}\right) \in \widetilde{\mathrm{B}}: \tilde{y} \widetilde{\nabla_{\theta} \varphi}\left(y_{0}, \gamma_{0}\right)=0\right\} .
$$

Proof. By Proposition 1.20 , we have that $\Phi$ is elliptic if and only if $\iota_{\mathrm{SG}}^{2,2}(\Phi)$ is nowhere vanishing on $\widetilde{\mathrm{B}}$. We rewrite this as

$$
\begin{aligned}
\iota_{\mathrm{SG}}^{2,2}(\Phi)(y, \gamma) & =\left.\tilde{\gamma}^{2} \tilde{y}^{2}\left(\langle x\rangle^{2}\left|\nabla_{x} \varphi(x, \theta)\right|^{2}+\langle\theta\rangle^{2}\left|\nabla_{\theta} \varphi(x, \theta)\right|^{2}\right)\right|_{(x, \theta)=\left(\iota^{-1}(y), \iota^{-1}(\gamma)\right)} \\
& =\left[\mid\left(\left.\iota_{\mathrm{SG}}^{1,0}(\langle x\rangle) \cdot \iota_{\mathrm{SG}}^{0,1}\left(\nabla_{x} \varphi\right)\right|^{2}+\mid\left(\left.\iota_{\mathrm{SG}}^{0,1}(\langle\theta\rangle) \cdot \iota_{\mathrm{SG}}^{1,0}\left(\nabla_{\theta} \varphi\right)\right|^{2}\right](y, \gamma)\right.\right.
\end{aligned}
$$

The maps $\left(\iota_{\mathrm{SG}}^{1,0}(\langle x\rangle)^{2}\right.$ and $\left(\iota_{\mathrm{SG}}^{0,1}(\langle\theta\rangle)\right)^{2}$ are nowhere vanishing, since the maps $(x, \theta) \mapsto$ $\langle x\rangle$ and $(x, \theta) \mapsto\langle\theta\rangle$ are elliptic and polyhomogeneous (see Example 1.11), which proves the first assertion.

The characterization of $\widetilde{\mathcal{C}}_{\varphi}$ is a consequence of the same argument carried out for the second summand by itself, that is $\left|\langle\theta\rangle \nabla_{\theta} \varphi\right|^{2}$, in view of Definition 3.14.

We now seek to obtain a similar characterization of $\tilde{\Lambda}_{\varphi}$. We do so by finding an analogue of the map $\lambda_{\varphi}$ defined in (3.4). For an SG-phase function, we may define $\lambda_{\varphi}: \mathbb{R}^{d} \times \mathbb{R}^{s} \rightarrow \mathbb{R}^{d} \times \mathbb{R}^{d}$ by $(x, \theta) \mapsto\left(x, \nabla_{x} \varphi(x, \theta)\right)$. In the following discussion, we translate this to a map $\left(\mathbb{B}^{d} \times \mathbb{B}^{s}\right)^{o} \rightarrow \mathbb{B}^{d} \times \mathbb{B}^{d}$ and then show that it extends to parts of the boundary $\widetilde{\mathrm{B}}$ in a neighbourhood of $\widetilde{\mathcal{C}}_{\varphi}$.

We thus start by considering the map $\left(\iota^{-1} \times \iota^{-1}\right)^{*} \lambda_{\varphi}=\left((y, \gamma) \mapsto\left(\iota^{-1} y, \widetilde{\nabla_{x} \varphi}\right)\right.$. By 
compactification of the image space to $\mathbb{B}^{d} \times \mathbb{B}^{d}$ we set

$$
\left.\tilde{\lambda}_{\varphi}\right|_{\left(\mathbb{B}^{d} \times \mathbb{B}^{d}\right)^{\circ}}=(\iota \times \iota) \circ\left(\left(\iota^{-1} \times \iota^{-1}\right)^{*} \lambda_{\varphi}\right)
$$

We now study the extendability of this map to parts of the boundary $\widetilde{\mathrm{B}}$, that is to

$$
\begin{aligned}
\widetilde{\mathcal{E}} & =\left(\left(\mathbb{B}^{d}\right)^{o} \times\left(\mathbb{B}^{s}\right)^{o}\right) \sqcup \widetilde{\mathrm{B}}^{e} \sqcup \widetilde{\mathrm{B}}_{\mathrm{ell}}, \\
\widetilde{\mathrm{B}}_{\mathrm{ell}} & =\left\{\left(y_{0}, \gamma_{0}\right) \in \widetilde{\mathrm{B}}^{\psi} \cup \widetilde{\mathrm{B}}^{\psi e}:\left|\nabla_{x} \varphi\right|^{2} \text { is elliptic at }\left(y_{0}, \gamma_{0}\right)\right\} .
\end{aligned}
$$

Remark 3.20. This definition of $\tilde{\lambda}_{\varphi}$ may be illustrated in the following commutative diagram.

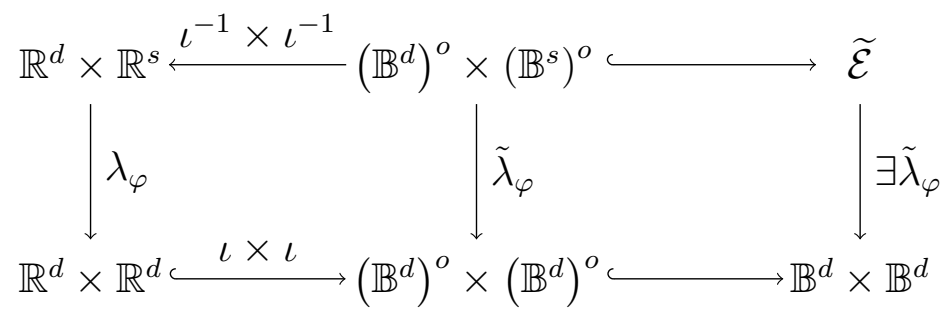

We will prove the extendability of $\tilde{\lambda}_{\varphi}$ in terms of that of the map $\left(\iota_{\mathrm{SG}}^{1,0} \times \iota_{\mathrm{SG}}^{0,1}\right) \lambda_{\varphi}$ : $\mathbb{B}^{d} \times \mathbb{B}^{s} \rightarrow \mathbb{B}^{d} \times \mathbb{R}^{d}$, which is given by

$$
(y, \gamma) \mapsto\left(y, \tilde{\gamma} \widetilde{\nabla_{x} \varphi}(y, \gamma)\right)
$$

and is smooth up to the boundary by Proposition 1.20. We will show that, close to the boundary components of $\widetilde{\mathcal{E}}$, this property yields the desired extension of $\tilde{\lambda}_{\varphi}$.

Proposition 3.21. The map $\tilde{\lambda}_{\varphi}$, defined on $\left(\mathbb{B}^{d}\right)^{o} \times\left(\mathbb{B}^{s}\right)^{o}$ by $(3.25)$, admits a smooth extension to the subset $\widetilde{\mathcal{E}} \subset \mathbb{B}^{d} \times \mathbb{B}^{s}$ defined in 3.26 .

Proof. We prove this as in [49] by considering each component of $\widetilde{\mathcal{E}}$ individually. Since the first component of $\tilde{\lambda}_{\varphi}$ coincides with the projection on the first set of variables $\operatorname{pr}_{1}$, that is $\operatorname{pr}_{1} \circ \tilde{\lambda}_{\varphi}=\operatorname{pr}_{1}$, it is smoothly extendible from the interior to the whole of $\mathbb{B}^{d} \times \mathbb{B}^{s}$. Consequently it is enough to consider the second component of $\tilde{\lambda}_{\varphi}$.

Since $\iota$ is a diffeomorphism between $\mathbb{R}^{d}\left(\right.$ or $\left.\mathbb{R}^{s}\right)$ and $\left(\mathbb{B}^{d}\right)^{o}$ (or $\left.\left(\mathbb{B}^{s}\right)^{o}\right), \tilde{\lambda}_{\varphi}$ is smooth in the interior, i.e. on $\left(\mathbb{B}^{d}\right)^{o} \times\left(\mathbb{B}^{s}\right)^{o}$ as a composition of smooth maps.

Consequently it is enough to consider (3.25) for $|y|>2 / 3$ or $|\gamma|>2 / 3$. We first consider this map near the interior of the $e$-face $\widetilde{\mathrm{B}}^{e}$, i.e. for $|y|>2 / 3$ with $|\gamma|<c<1$. Here we write, using 3.23 and Proposition 1.18 , for some vector-valued 
symbol $p \in \mathrm{SG}^{-1,1}$,

$$
\begin{aligned}
\iota\left(\widetilde{\nabla_{x} \varphi}(y, \gamma)\right) & =\iota\left(\nabla_{x} \varphi^{e}\left(\frac{y}{|y|}(1-|y|)^{-1}, \frac{\gamma}{|\gamma|}(1-|\gamma|)^{-1}\right)+\left(\iota^{-1} \times \iota^{-1}\right)^{*} p(y, \gamma)\right) \\
& =\iota\left(\nabla_{x} \varphi^{e}\left(\frac{y}{|y|}, \frac{\gamma}{|\gamma|}(1-|\gamma|)^{-1}\right)+\left(\iota^{-1} \times \iota^{-1}\right)^{*} p(y, \gamma)\right) .
\end{aligned}
$$

where in the second step we have used the 0-homogeneity of $\nabla_{x} \varphi^{e}$ in the first set of variables. Using this expression, we now extend $\tilde{\lambda}_{\varphi}$ smoothly to

$$
\widetilde{A}_{1}=\left\{y \in \mathbb{B}^{d}: 2 / 3<|y| \leq 1\right\} \times\left\{\gamma \in \mathbb{B}^{s}:|\gamma|<r^{\prime}\right\}
$$

with arbitrary $r^{\prime}, 1>r^{\prime}>2 / 3$. In fact, this is clearly possible for the first summand in the argument of $\iota$ in the right hand side of 3.28). For the second summand, Proposition 1.20 grants that for each vector component of $p$, that is $p_{j} \in \mathrm{SG}^{-1,1}\left(\mathbb{R}^{d} \times \mathbb{R}^{s}\right)$, that

$$
\left(\iota^{-1} \times \iota^{-1}\right)^{*} p_{j} \in \tilde{y} \tilde{\gamma}^{-1} \mathscr{C}^{\infty}\left(\mathbb{B}^{d} \times \mathbb{B}^{s}\right) \subset \tilde{\gamma}^{-1} \mathscr{C}^{\infty}\left(\mathbb{B}^{d} \times \mathbb{B}^{s}\right)
$$

and consequently $\left(\iota^{-1} \times \iota^{-1}\right)^{*} p$ is smooth on $\widetilde{A}_{1}$ as well. Moreover, the values of both such extensions to $\widetilde{A}_{1}$ remain bounded, and $\iota$ is smooth on $\mathbb{R}^{d}$. This implies the extendability of $\tilde{\lambda}_{\varphi}$ to any point in $\widetilde{\mathrm{B}}^{e}$.

We now consider the subset of $\mathbb{B}^{d} \times \mathbb{B}^{s}$ given by

$$
\widetilde{A}_{2}=\left\{y \in \mathbb{B}^{d}: 2 / 3<|y| \leq 1\right\} \times\left\{\gamma \in \mathbb{B}^{s}:|\gamma|>r\right\},
$$

$r^{\prime}>r>2 / 3$. Observe that we have $\widetilde{\mathrm{B}}_{\text {ell }} \subset \widetilde{A}_{2}$. Again we may write (close to the boundary) for a vector-valued symbol $q \in \mathrm{SG}^{0,0}\left(\mathbb{R}^{d} \times \mathbb{R}^{s}\right)$,

$$
\begin{aligned}
\iota\left(\widetilde{\nabla_{x} \varphi}(y, \gamma)\right) & =\iota\left(\nabla_{x} \varphi^{\psi}\left(\frac{y}{|y|}(1-|y|)^{-1}, \frac{\gamma}{|\gamma|}(1-|\gamma|)^{-1}\right)+\left(\iota^{-1} \times \iota^{-1}\right)^{*} q(y, \gamma)\right) \\
& =\iota\left(\nabla_{x} \varphi^{\psi}\left(\frac{y}{|y|}(1-|y|)^{-1}, \frac{\gamma}{|\gamma|}\right)(1-|\gamma|)^{-1}+\left(\iota^{-1} \times \iota^{-1}\right)^{*} q(y, \gamma)\right) .
\end{aligned}
$$

By Proposition $1.20,\left(\iota^{-1} \times \iota^{-1}\right)^{*} q=\iota_{\mathrm{SG}}^{0,0}(q)$ extends smoothly to $\mathbb{B}^{d} \times \mathbb{B}^{s}$ and in particular stays bounded. 
Due to ellipticity, we have at points $\left(y_{0}, \gamma_{0}\right) \in \widetilde{\mathrm{B}}_{\mathrm{ell}}$, by Proposition 1.18 and 1.20 .

$$
\begin{aligned}
\text { either } \quad\left(y_{0}, \gamma_{0}\right) & \in \widetilde{\mathrm{B}}^{\psi} \text { and } \nabla_{x} \varphi^{\psi}\left(\iota^{-1}\left(y_{0}\right), \gamma_{0}\right) \neq 0, \\
\text { or } \quad\left(y_{0}, \gamma_{0}\right) & \in \widetilde{\mathrm{B}}^{\psi e} \text { and } \nabla_{x} \varphi^{\psi e}\left(y_{0}, \gamma_{0}\right) \neq 0 .
\end{aligned}
$$

In the former case, the norm of the first summand in the argument of $\iota$ in the right hand side of $(3.29)$ tends to $+\infty$ when $|\gamma| \nearrow 1$. Then, sufficiently close to $\left(y_{0}, \gamma_{0}\right)$, we have

$$
\iota\left(\widetilde{\nabla_{x} \varphi}\right)=\frac{\widetilde{\nabla_{x} \varphi}}{\left|\widetilde{\nabla_{x} \varphi}\right|}\left(1-\frac{1}{\left|\widetilde{\nabla_{x} \varphi}\right|}\right)=\frac{\tilde{\gamma} \widetilde{\nabla_{x} \varphi}}{\left|\tilde{\gamma} \widetilde{\nabla_{x} \varphi}\right|}\left(1-\frac{\tilde{\gamma}}{\left|\tilde{\gamma} \widetilde{\nabla_{x} \varphi}\right|}\right)
$$

where $\tilde{\gamma} \widetilde{\nabla_{x} \varphi}=\iota_{\mathrm{SG}}^{0,1}\left(\nabla_{x} \varphi\right)$ is smooth up to the boundary, granted by Proposition 1.20. Moreover, by homogeneity, we can write

$\tilde{\gamma} \widetilde{\nabla_{x} \varphi}(y, \gamma)=\tilde{\gamma}\left(\iota^{-1} \times \iota^{-1}\right)^{*} \nabla_{x} \varphi(y, \gamma)=\nabla_{x} \varphi^{\psi}\left(\frac{y}{|y|}(1-|y|)^{-1}, \frac{\gamma}{|\gamma|}\right)+\tilde{\gamma} \iota_{\mathrm{SG}}^{0,0} q(y, \gamma)$.

Consequently this expression cannot vanish close to $\left(y_{0}, \gamma_{0}\right)$, since by ellipticity $\left|\nabla_{x} \varphi^{\psi}\left(\iota^{-1}\left(y_{0}\right), \gamma_{0}\right)\right| \neq 0$ and we have (since $\tilde{\gamma}$ vanishes on the $\psi$-boundary) $\left|\tilde{\gamma} \iota_{\mathrm{SG}}^{0,0} q(y, \gamma)\right|=0$. The smooth extendibility of 3.30 to points in $\widetilde{\mathrm{B}}_{\text {ell }} \cap \widetilde{\mathrm{B}}^{\psi}$ follows. The remaining case of points at the corner, that is the result for $\left(y_{0}, \gamma_{0}\right) \in \widetilde{\mathrm{B}}_{\text {ell }} \cap \widetilde{\mathrm{B}}^{\psi e}$, follows in a similar way, writing by homogeneity

$$
\begin{aligned}
\iota\left(\widetilde{\nabla_{x} \varphi}(y, \gamma)\right) & =\iota\left(\left(\iota^{-1} \times \iota^{-1}\right)^{*} \nabla_{x} \varphi(y, \gamma)\right) \\
& =\iota\left(\nabla_{x} \varphi^{\psi e}\left(\frac{y}{|y|}, \frac{\gamma}{|\gamma|}\right)(1-|\gamma|)^{-1}+\left(\iota^{-1} \times \iota^{-1}\right)^{*}(p+q)(y, \gamma)\right)
\end{aligned}
$$

with $p \in \mathrm{SG}^{-1,1}, q \in \mathrm{SG}^{0,0}$ and $\nabla_{x} \varphi\left(y_{0}, \gamma_{0}\right) \neq 0$, so that

$$
\tilde{\gamma}\left(\widetilde{\nabla_{x} \varphi}(y, \gamma)=\nabla_{x} \varphi^{\psi e}\left(\frac{y}{|y|}, \frac{\gamma}{|\gamma|}\right)+\tilde{\gamma}\left(\iota^{-1} \times \iota^{-1}\right)^{*} p(y, \gamma)+\tilde{\gamma}\left(\iota^{-1} \times \iota^{-1}\right)^{*} q(y, \gamma) .\right.
$$

All summands are smoothly extendible to $\left(y_{0}, \gamma_{0}\right)$, the latter two even vanishing there. The proof is complete.

Note that the extendibility of $\tilde{\lambda}_{\varphi}$ to $\widetilde{\mathcal{E}}$, in view of Lemma 3.19 , includes that $\tilde{\lambda}_{\varphi}$ is well defined in a neighbourhood of $\widetilde{\mathcal{C}}_{\varphi}$. Indeed, by the characterization of $\widetilde{\mathcal{C}}_{\varphi}$ in terms of vanishing of $\tilde{y} \widetilde{\nabla_{\theta} \varphi}$, at points $\left(y_{0}, \gamma_{0}\right) \in \widetilde{\mathcal{C}}_{\varphi}$ we necessarily have 
$\tilde{\gamma} \nabla_{y} \tilde{\varphi}\left(y_{0}, \gamma_{0}\right) \neq 0$ and consequently the same holds, by continuity, in a neighbourhood of $\left(y_{0}, \gamma_{0}\right)$ in $\widetilde{B}$.

We may now characterize $\tilde{\Lambda}_{\varphi}$ in terms of $\tilde{\lambda}_{\varphi}$ and $\widetilde{\mathcal{C}}_{\varphi}$, in analogy to (3.5).

Lemma 3.22. Let $\varphi \in \mathrm{SG}_{\mathrm{cl}}^{1,1}\left(\mathbb{R}^{d} \times \mathbb{R}^{s}\right)$ be a classical SG-phase function. Then, we have $\tilde{\Lambda}_{\varphi}=\tilde{\lambda}_{\varphi}\left(\widetilde{\mathcal{C}}_{\varphi}\right)$.

For the sake of brevity, we omit the details of the proof, which is achieved by use of the same methods used in the proof of Proposition 3.21 above and Lemma 1.4 .

We have characterized the sets $\widetilde{\mathcal{C}}_{\varphi}$ and $\tilde{\Lambda}_{\varphi}$ associated to a phase function $\varphi$ as subsets of the boundary of the corresponding compactifications. As in [50], we will now make a non-degeneracy assumption on the phase function that allows us to study these objects in the framework of the theory of analysis on manifolds with corners. As mentioned, [98] serves as our main reference for this theory, consider also the unpublished [105]. An overview of the concepts we use in the following may also be found in the appendix of [50].

Definition 3.23 (Non-degenerate classical SG-phase functions).

Let $\varphi \in \mathrm{SG}_{\mathrm{cl}}^{1,1}\left(\mathbb{R}^{d} \times \mathbb{R}^{s}\right)$ be a classical SG-phase function. Then $\varphi$ is called nondegenerate if the differentials $\left\{d\left(\left.\tilde{y} \widetilde{\partial_{\theta_{j}} \varphi}\right|_{X}\right)\right\}_{j=1, \ldots, s}$ form, for every $\left(y_{0}, \gamma_{0}\right) \in \widetilde{\mathcal{C}_{\varphi}}$, a set of linearly independent vectors in $T_{\left(y_{0}, \gamma_{0}\right)}^{*} X$, where the placeholder $X$ may be replaced by all possible boundary and corner components of $\mathbb{B}^{d} \times \mathbb{B}^{s}$, that is,

$$
X \in\left\{\widetilde{\mathrm{B}}^{e}, \widetilde{\mathrm{B}}^{\psi}, \widetilde{\mathrm{B}}^{\psi e}\right\} .
$$

This definition precisely guarantees that $\tilde{y} \widetilde{\nabla_{\theta} \varphi}$ fulfils the assumptions of the regular value theorem for manifolds with corners as stated in [98, Prop. 4.2.10] on $\widetilde{\mathcal{C}}_{\varphi}$, which by Lemma 3.19 is defined as $\left(\tilde{y} \widetilde{\nabla_{\theta} \varphi}\right)^{-1}\{0\}$.

Before we formulate the consequences of this theorem as in [50], we recall that a totally neat submanifold $N$ of a manifold with boundary $M$ is simply a submanifold with boundary such that $\partial N \subset \partial M$. Two submanifolds $N_{1}$ and $N_{2}$ of some manifold $M$ are said to intersect cleanly (consider [83, App. C.3.]) if for every $\left(y_{0}, \gamma_{0}\right) \in\left(M_{1} \cap M_{2}\right)$ we have for some $e>0$ (called the excess)

$$
\begin{aligned}
T_{\left(y_{0}, \gamma_{0}\right)}\left(N_{1} \cap N_{2}\right) & =T_{\left(y_{0}, \gamma_{0}\right)} N_{1} \cap T_{\left(y_{0}, \gamma_{0}\right)} N_{2} . \\
\operatorname{codim}_{M}\left(N_{1}\right)+\operatorname{codim}_{M}\left(N_{2}\right) & =\operatorname{codim}_{M}\left(N_{1} \cap N_{2}\right)+e
\end{aligned}
$$


Indeed, each of the boundary faces $\widetilde{\mathrm{B}}^{e}$ and $\widetilde{\mathrm{B}}^{\psi}$ are submanifolds (with boundary) of the manifold with corners $\mathbb{B}^{d} \times \mathbb{B}^{s}$ that intersect cleanly at their joint boundary $\widetilde{\mathrm{B}}^{\psi e}$. For the components of $\widetilde{\mathcal{C}}_{\varphi}$ situated in these boundary faces, we find a similar set-up.

Proposition 3.24. Let $\varphi \in \mathrm{SG}_{\mathrm{cl}}^{1,1}\left(\mathbb{R}^{d} \times \mathbb{R}^{s}\right)$ be a non-degenerate SG-phase function. Then $\widetilde{\mathcal{C}}_{\varphi}$ has the following properties.

(1) The different components of $\widetilde{\mathcal{C}}_{\varphi}$ are totally neat submanifolds of the corresponding boundary component of $\mathbb{B}^{d} \times \mathbb{B}^{s}$. In particular we have

$$
\widetilde{\mathcal{C}}_{\varphi}=\underbrace{\widetilde{\mathcal{C}}_{\varphi}^{\psi}}_{\subset \widetilde{\mathrm{B}}^{\psi}} \cup \underbrace{\widetilde{\mathcal{C}}_{\varphi}^{e}}_{\subset \widetilde{\mathrm{B}}^{e}},
$$

and their possible boundaries form a subset $\widetilde{\mathcal{C}}_{\varphi}^{\psi e}$ of $\widetilde{\mathrm{B}}^{\psi e}$.

(2) The codimension of the respective component is always s, i.e. $\operatorname{dim}\left(\widetilde{\mathcal{C}}_{\varphi}^{e}\right)=$ $\operatorname{dim}\left(\widetilde{\mathcal{C}}_{\varphi}^{\psi}\right)=d-1$ and (if non-empty) $\operatorname{dim}\left(\widetilde{\mathcal{C}}_{\varphi}^{\psi e}\right)=d-2$.

(3) The tangent space to each face of $\widetilde{\mathcal{C}}_{\varphi}^{\bullet}$ in $\widetilde{\mathrm{B}}^{\bullet}$ may be calculated as

$$
\left\{v \in T_{\left(y_{0}, \gamma_{0}\right)}\left(\widetilde{\mathrm{B}}^{\bullet}\right) \mid\left(d_{y, \gamma}\left(\left.\tilde{y} \widetilde{\partial_{\theta_{j}} \varphi}\right|_{\widetilde{\mathrm{B}}}\right)\right) v=0 \forall j \in\{1, \ldots, s\}\right\} .
$$

As a consequence of these properties, the intersection $\widetilde{\mathcal{C}}_{\varphi}^{\psi} \cap \widetilde{\mathcal{C}}_{\varphi}^{e}=\widetilde{\mathcal{C}}_{\varphi}^{\psi e}$ is clean.

Since $\tilde{\lambda}_{\varphi}$ is smooth up to the boundary in a neighbourhood of $\widetilde{\mathcal{C}}_{\varphi}$, we obtain a similar statement for its image $\tilde{\Lambda}_{\varphi}$.

Proposition 3.25. Let $\varphi \in \mathrm{SG}_{\mathrm{cl}}^{1,1}\left(\mathbb{R}^{d} \times \mathbb{R}^{s}\right)$ be a non-degenerate SG-phase function. Then $\tilde{\Lambda}_{\varphi}$ has the following properties.

(1) The different components of $\tilde{\Lambda}_{\varphi}$ are each totally neat submanifolds of the corresponding boundary component of $\mathbb{B}^{d} \times \mathbb{B}^{s}$. In particular we have

$$
\tilde{\Lambda}_{\varphi}=\underbrace{\tilde{\Lambda}_{\varphi}^{\psi}}_{\subset \widetilde{\mathrm{W}}_{\mathrm{SG}}^{\psi}} \cup \underbrace{\tilde{\Lambda}_{\varphi}^{e}}_{\subset \widetilde{\mathrm{W}}_{\mathrm{SG}}^{e}}
$$

and their possible boundaries form a subset $\tilde{\Lambda}_{\varphi}^{\psi e}$ of $\widetilde{\mathrm{W}}_{\mathrm{SG}}^{\psi e}$.

(2) The codimension of the respective component is always $d$, i.e. $\operatorname{dim}\left(\tilde{\Lambda}_{\varphi}^{e}\right)=$ $\operatorname{dim}\left(\tilde{\Lambda}_{\varphi}^{\psi}\right)=d-1$ and (if non-empty) $\operatorname{dim}\left(\tilde{\Lambda}_{\varphi}^{\psi e}\right)=d-2$. 
(3) The tangent space to each face of $\tilde{\Lambda}_{\varphi}^{\bullet}$ in $\widetilde{\mathrm{W}}_{\mathrm{SG}}$ may be calculated by means of the differential of $\tilde{\lambda}_{\varphi}$, that is, via

$$
T \tilde{\Lambda}_{\varphi}^{\bullet}=\left(d\left(\left.\tilde{\lambda}_{\varphi}\right|_{\widetilde{\mathcal{C}}_{\varphi}}\right)\right) T \widetilde{\mathcal{C}}_{\varphi}^{\bullet}
$$

(4) The intersection $\tilde{\Lambda}_{\varphi}^{\psi} \cap \tilde{\Lambda}_{\varphi}^{e}=\tilde{\Lambda}_{\varphi}^{\psi e}$ is clean.

As in [50, Fig. 1], this geometric setup of clean intersection may be schematically visualized, see Figure 12. Therein, since we are limited to 3 dimensions, the variables parallel to the corner, $\left(y^{\|}, \eta^{\|}\right)$, are projected into one.

Having established the geometric set-up on the compactifications of $\mathbb{R}^{d} \times \mathbb{R}^{s}$

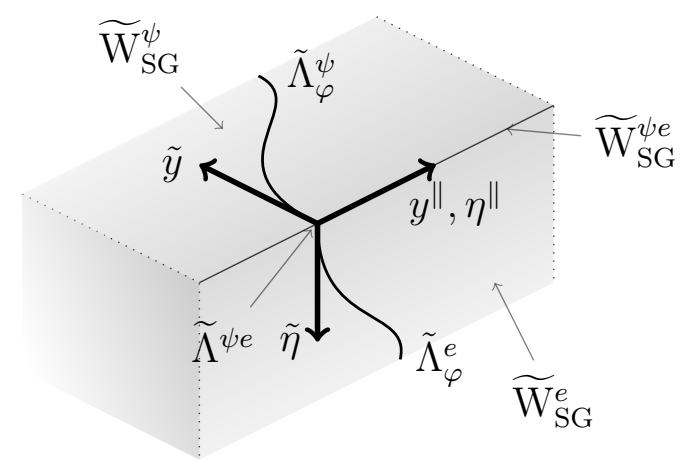

FIGURE 12. Intersection of $\tilde{\Lambda}_{\varphi}^{\psi} \subset \widetilde{\mathrm{W}}_{\mathrm{SG}}^{\psi}$ and $\tilde{\Lambda}_{\varphi}^{e} \subset \widetilde{\mathrm{W}}_{\mathrm{SG}}^{e}$ at the corner $\widetilde{\mathrm{W}}_{\mathrm{SG}}^{\psi e}$

and $\mathbb{R}^{d} \times \mathbb{R}^{d}$, we now establish, as in [50], a characterization of $\widetilde{\mathcal{C}}_{\varphi}$ and $\tilde{\Lambda}_{\varphi}$ in terms of the different components of the principal symbol of $\varphi$. As in the classical theory recalled in Section 3.2.1, the homogeneity of the different components of $\sigma(\varphi)$ has as a consequence that the associated submanifolds are conic. This can be formulated using the map $\Gamma$ introduced in Construction 1.1 .

Lemma 3.26. Let $\varphi \in \mathrm{SG}_{\mathrm{cl}}^{1,1}\left(\mathbb{R}^{d} \times \mathbb{R}^{s}\right)$ be a classical SG-phase function. Then, we have

$$
\begin{aligned}
\mathcal{C}_{\varphi}^{\psi} & :=\left(\iota^{-1} \times \Gamma\right)\left(\widetilde{\mathcal{C}}_{\varphi} \cap \widetilde{\mathrm{B}}^{\psi}\right)=\left\{\left(x_{0}, \theta_{0}\right) \in \mathrm{B}^{\psi}: \nabla_{\theta} \varphi^{\psi}\left(x_{0}, \theta_{0}\right)=0\right\}, \\
\mathcal{C}_{\varphi}^{e} & :=\left(\Gamma \times \iota^{-1}\right)\left(\widetilde{\mathcal{C}}_{\varphi} \cap \widetilde{\mathrm{B}}^{e}\right)=\left\{\left(x_{0}, \theta_{0}\right) \in \mathrm{B}^{e}: \nabla_{\theta} \varphi^{e}\left(x_{0}, \theta_{0}\right)=0\right\}, \\
\mathcal{C}_{\varphi}^{\psi e} & :=(\Gamma \times \Gamma)\left(\widetilde{\mathcal{C}}_{\varphi} \cap \widetilde{\mathrm{B}}^{\psi e}\right)=\left\{\left(x_{0}, \theta_{0}\right) \in \mathrm{B}^{\psi e}: \nabla_{\theta} \varphi^{\psi e}\left(x_{0}, \theta_{0}\right)=0\right\} .
\end{aligned}
$$

Proof. By Definition 3.14,

$$
\widetilde{\mathcal{C}}_{\varphi}=\left\{\left.\left(y_{0}, \gamma_{0}\right) \in \widetilde{\mathrm{B}}|| \nabla_{\theta} \varphi\right|^{2} \text { is not elliptic at }\left(y_{0}, \gamma_{0}\right)\right\} \text {. }
$$


By the characterization of ellipticity of Proposition 1.20, we have that $\left|\nabla_{\theta} \varphi\right|^{2}$ is elliptic at $\left(y_{0}, \gamma_{0}\right) \in \widetilde{\mathrm{B}}$ if and only if the corresponding principal symbol is nonvanishing at the corresponding point $\left(x_{0}, \theta_{0}\right) \in \mathrm{B}$. By Proposition 1.18 , we have

$$
\sigma_{\bullet}\left(\left|\nabla_{\theta} \varphi\right|^{2}\right)=\sigma_{\bullet}\left(\sum_{j=1}^{s}\left|\partial_{\theta_{j}} \varphi\right|^{2}\right)=\sum_{j=1}^{s}\left|\partial_{\theta_{j}} \sigma_{\bullet}(\varphi)\right|^{2}=\sum_{j=1}^{s}\left|\partial_{\theta_{j}} \varphi^{\bullet}\right|^{2},
$$

for any of the labels $\bullet \in\{\psi, e, \psi e\}$ of the principal symbol, and the assertion follows.

Remark 3.27. Note that the $\mathcal{C}_{\varphi}^{\psi}$-component coincides with the standard notion $\mathcal{C}_{\varphi}$ for a homogenous (classical) phase function $\varphi^{\psi}$, as in (3.3).

Similarly to Lemma 3.26, we obtain a characterization of the different components of $\tilde{\Lambda}_{\varphi}$.

Lemma 3.28. Define the triple $\left(\Lambda_{\varphi}^{\psi}, \Lambda_{\varphi}^{e}, \Lambda_{\varphi}^{\psi e}\right) \subset\left(\mathrm{W}_{\mathrm{SG}}^{\psi}, \mathrm{W}_{\mathrm{SG}}^{e}, \mathrm{~W}_{\mathrm{SG}}^{\psi e}\right)$ by

$$
\Lambda_{\varphi}^{\bullet}:=\left\{\left(\left(x, \nabla_{x} \varphi^{\bullet}(x, \theta)\right) \mid \exists(x, \theta) \in \mathrm{B}^{\bullet}: \nabla_{\theta} \varphi^{\bullet}(x, \theta)=0\right\}\right.
$$

for $\bullet\{\{, e, \psi e\}$. Then we have

$$
\Lambda_{\varphi}^{\psi}=\left(\iota^{-1} \times \Gamma\right)\left(\tilde{\Lambda}_{\varphi}^{\psi}\right), \Lambda_{\varphi}^{e}=\left(\Gamma \times \iota^{-1}\right)\left(\tilde{\Lambda}_{\varphi}^{e}\right) \text { and } \Lambda_{\varphi}^{\psi e}=(\Gamma \times \Gamma)\left(\tilde{\Lambda}_{\varphi}^{\psi e}\right) .
$$

Proof. We start with the proof for $\Lambda_{\varphi}^{\psi}$, which coincides with the classical definition of the manifold of stationary points for a classical homogeneous phase function 3.5. By Lemma 3.22, we may obtain $\tilde{\Lambda}_{\varphi}^{\psi}$ as $\tilde{\lambda}_{\varphi}\left(\widetilde{\mathcal{C}}_{\varphi}^{\psi}\right)$ and thus we may write

$$
\left(\iota^{-1} \times \Gamma\right)\left(\tilde{\Lambda}_{\varphi}^{\psi}\right)=\left[\left(\iota^{-1} \times \Gamma\right) \circ \tilde{\lambda}_{\varphi}\right]\left(\widetilde{\mathcal{C}}_{\varphi}^{\psi}\right) .
$$

By Lemma 3.19 we have $\tilde{y} \widetilde{\nabla_{\theta} \varphi}(y, \gamma)=0$ on $\widetilde{\mathcal{C}_{\varphi}}$ and consequently, in view of the absence of critical points characterized in same Lemma, $\widetilde{\gamma} \widetilde{\nabla_{x}} \varphi(y, \gamma) \neq 0$.

Revisiting the explicit form of $\tilde{\lambda}_{\varphi}$ near $\widetilde{\mathcal{C}}_{\varphi}^{\psi}$, that is (3.30) from the proof of Proposition 3.21 , and using the fact that $\tilde{\gamma}$ vanishes on $\widetilde{\mathcal{C}}_{\varphi}^{\psi}$, we can write

$$
\begin{aligned}
\left(\iota^{-1} \times \Gamma\right)\left(\tilde{\Lambda}_{\varphi}^{\psi}\right) & =\left\{\left(\left(\iota^{-1}(y), \mu \frac{\tilde{\gamma} \widetilde{\nabla_{x} \varphi}(y, \gamma)}{\left|\widetilde{\gamma} \widetilde{\nabla_{x} \varphi}(y, \gamma)\right|}\right) \mid(y, \gamma) \in \widetilde{\mathcal{C}}_{\varphi}^{\psi}, \mu>0\right\}\right. \\
& =\left\{\left(\left(x, \mu \frac{\nabla_{x} \varphi^{\psi}(x, \theta)}{\left|\nabla_{x} \varphi^{\psi}(x, \theta)\right|}\right) \mid(x, \theta) \in \mathcal{C}_{\varphi}^{\psi}, \mu>0\right\},\right.
\end{aligned}
$$


where we have made use of the characterization of the principal symbol by evaluation at the boundary in Proposition 1.20 and where we have commuted differentiation and the principal symbol map as granted by Proposition 1.18. Making use of the homogeneity of $\varphi^{\psi}$, we may write this simply as

$$
\left(\iota^{-1} \times \Gamma\right)\left(\tilde{\Lambda}_{\varphi}^{\psi}\right)=\left\{\left(\left(x, \nabla_{x} \varphi^{\psi}(x, \theta)\right) \mid(x, \theta) \in \mathbb{R}^{d} \times\left(\mathbb{R}^{s} \backslash 0\right) \text { and } \nabla_{\theta} \varphi^{\psi}(x, \theta)=0\right\},\right.
$$

which is the definition of $\Lambda_{\varphi}^{\psi}$, as claimed.

Making use of the same techniques, we write for the $e$-component

$$
\begin{aligned}
\left(\Gamma \times \iota^{-1}\right)\left(\tilde{\Lambda}_{\varphi}^{e}\right) & \left.=\left[\Gamma \times \iota^{-1}\right) \tilde{\lambda}_{\varphi}\right]\left(\widetilde{\mathcal{C}}_{\varphi}^{e}\right) \\
& =\left\{\left(\mu y, \widetilde{\nabla_{x} \varphi}(y, \gamma)\right):(y, \gamma) \in \widetilde{\mathcal{C}}_{\varphi}^{e}\right\} \\
& =\left\{\left(x, \nabla_{x} \varphi^{e}(x, \theta)\right):(x, \theta) \in \mathcal{C}_{\varphi}^{e}\right\}
\end{aligned}
$$

where we have again obtained the principal symbol by evaluation on the boundary, as in Proposition 1.20 .

We may obtain the characterization of the corner component $\Lambda_{\varphi}^{\psi e}$ in exactly the same way.

As mentioned in Proposition 3.3, a fundamental property of $\Lambda_{\varphi}^{\psi}$, as defined in Lemma 3.28 , is that it is a conic Lagrangian submanifold of $\mathbb{R}^{d} \times\left(\mathbb{R}^{d} \backslash\{0\}\right)$. We recall that a closed $d$-dimensional submanifold $\Lambda^{\psi}$ of $T^{*} \mathbb{R}^{d} \backslash\{0\}=\mathbb{R}^{d} \times$ $\left(\mathbb{R}^{d} \backslash\{0\}\right)$ is called conic Lagrangian if one of the following equivalent conditions is met

- $\Lambda^{\psi}$ is conic in the second variable and the symplectic two-form $\omega$ vanishes over it.

- The tautological 1-form on $\mathbb{R}^{d} \times\left(\mathbb{R}^{d} \backslash\{0\}\right) \cong T^{*} \mathbb{R}^{d} \backslash\{0\}$ vanishes on $\Lambda^{\psi}$.

We refer to Chapter 3.7. of [55] for a proof of this equivalence. In what follows, we establish an analogous statement for $\Lambda^{e}$, as in [50].

From the discussion in [55], we deduce that the two formulations are equivalent by noticing that (using local canonical coordinates)

$$
\begin{gathered}
d \alpha^{\psi}=d(\xi d x)=d \xi \wedge d x=\omega \quad \text { and } \\
i_{\varrho^{\psi} \omega} \omega(\cdot)=(d \xi \wedge d x)\left(\xi \cdot \partial_{\xi}, \cdot\right)=\xi d x=\alpha^{\psi}(\cdot),
\end{gathered}
$$

where the (radial) vector field $\varrho^{\psi}=\xi \cdot \partial_{\xi}$ can be invariantly obtained, consider [83, Sect. 21.1], by defining it as the generator of the dilation in the co-variable, that 
is by

$$
\varrho^{\psi}(f)=\left.\frac{d}{d \mu} f(\cdot, \mu \cdot)\right|_{\mu=1} \quad f \in \mathscr{C}^{\infty}\left(T^{*} M \backslash\{0\}\right)
$$

When $M=\mathbb{R}^{d} \backslash\{0\}$ is the (flat) Euclidean space (with 0 removed), we also may dilate in the variable, and define a similar vector field.

Definition 3.29. Define the (radial exit) vector field $\varrho^{e}$ on $T^{*}\left(\mathbb{R}^{d} \backslash\{0\}\right)$ by setting, for $f \in \mathscr{C}^{\infty}\left(T^{*}\left(\mathbb{R}^{d} \backslash\{0\}\right)\right), \varrho^{e}(f)=\left.\frac{d}{d \mu} f(\mu \cdot, \cdot)\right|_{\mu=1}$.

The exit-one-form on $T^{*}\left(\mathbb{R}^{d} \backslash\{0\}\right)$ is defined as

$$
\alpha^{e}:=-i_{\varrho^{e}} \omega .
$$

In local coordinates we have

$$
\varrho^{e}(f)=\left.\frac{d}{d \mu} f(\mu x, \xi)\right|_{\mu=1}=x \cdot\left(\nabla_{x} f\right) .
$$

In local canonical coordinates we may write this as $\alpha^{e}=-i_{\varrho^{e}} \omega=-x d \xi$, and therefore, again, $d \alpha^{e}=\omega$. We may specify in which sense $\Lambda_{\varphi}^{e}$ is a Lagrangian that is conic in the first set of variables.

Lemma 3.30. Let $\varphi$ be a non-degenerate classical SG-phase function. Then $\alpha^{e}$ vanishes on $\Lambda_{\varphi}^{e}$.

Remark 3.31. Before we prove this, we remark that Lemma 3.30 indeed requires its own proof, and cannot be simply "deduced by symmetry" from the classical theory, because of the "asymmetrical definition" of $\Lambda_{\varphi}$ with respect to $x$ and $\theta$.

Proof. This proof is again an excerpt from [49], and we adopt the notation of [55] for it, that is we denote for some coordinates $x$ on a manifold $M$ the induced coordinates on $T M$ by $(x, \delta x)$.

We first notice that $\Lambda_{\varphi}^{e}$ is, by its definition in Lemma 3.28, the image of

$$
\mathcal{C}_{\varphi}^{e}=\left\{\left(x_{0}, \theta_{0}\right) \in \mathbb{R}^{d} \backslash\{0\} \times \mathbb{R}^{s} \mid \nabla_{\theta} \varphi^{e}\left(x_{0}, \theta_{0}\right)=0\right\}
$$

under the map $\lambda_{\varphi}^{e}=\left(\operatorname{pr}_{1}, \nabla_{x} \varphi^{e}\right)$. By non-degeneracy of $\varphi$, this yields a smooth manifold and we may calculate its tangent space in terms of that of the preimage ${ }^{48}$ $T_{(x, \theta)} \mathcal{C}_{\varphi}^{e}$ is given by

$$
\left(\delta x \cdot \nabla_{x}\right) \nabla_{\theta} \varphi+\left(\delta \theta \cdot \nabla_{\theta}\right) \nabla_{\theta} \varphi=0,
$$

\footnotetext{
${ }^{48}$ In fact, as in Lemma 2.3.2 of [55], we can conclude from the upcoming $(3.32)$ and $(3.33)$ that $\left(\operatorname{pr}_{1}, \nabla_{x} \varphi^{e}\right)$ is an immersion, and thus its image is an immersed $d$-dimensional conic submanifold.
} 
and we thus have (denoting by $J$ the Jacobian)

$$
T_{\left(x, \nabla_{x} \varphi^{e}(x, \theta)\right)} \Lambda_{\varphi}^{e}=J\left(\operatorname{pr}_{1}, \nabla_{x} \varphi^{e}\right) \cdot T_{(x, \theta)} \mathcal{C}_{\varphi}^{e}
$$

Furthermore,

$$
J_{(x, \theta)}\left(\mathrm{pr}_{1}, \nabla_{x} \varphi^{e}\right)(\delta x, \delta \theta)=\left(\delta x,\left(\delta x \cdot \nabla_{x}\right) \nabla_{x} \varphi^{e}+\left(\delta \theta \cdot \nabla_{\theta}\right) \nabla_{x} \varphi^{e}\right) .
$$

Computing $\alpha^{e}=x \cdot d \xi$ on such a vector, we observe

$$
\begin{aligned}
x \cdot\left(\delta x \cdot \nabla_{x}\right) & \nabla_{x} \varphi^{e}+x \cdot\left(\delta \theta \cdot \nabla_{\theta}\right) \nabla_{x} \varphi^{e} \\
& =\sum_{j, k} x_{j}\left(\delta x_{k} \partial_{x_{k}}\right) \partial_{x_{j}} \varphi^{e}+\sum_{j}\left(\delta \theta \cdot \nabla_{\theta}\right) x_{j} \partial_{x_{j}} \varphi^{e} \\
& =\sum_{j, k}\left(\delta x_{k} \partial_{x_{k}}\right) x_{j} \partial_{x_{j}} \varphi^{e}-\sum_{k} \delta x_{k} \partial_{x_{k}} \varphi^{e}+\sum_{j}\left(\delta \theta \cdot \nabla_{\theta}\right) x_{j} \partial_{x_{j}} \varphi^{e} .
\end{aligned}
$$

By Euler's theorem for homogeneous functions applied to $\varphi^{e}$ this equals

$$
\begin{aligned}
(3.34) & =\sum_{k}\left(\delta x_{k} \partial_{x_{k}}\right) \varphi^{e}-\sum_{k} \delta x_{k} \partial_{x_{k}} \varphi^{e}+\left(\delta \theta \cdot \nabla_{\theta}\right) \varphi^{e}, \\
& =\delta \theta \cdot\left(\nabla_{\theta} \varphi^{e}\right) \stackrel{(x, \theta) \in \mathcal{C}_{\varphi}^{e}}{=} 0,
\end{aligned}
$$

which proves the assertion.

By now we have established the symplectic properties of $\Lambda_{\varphi}^{e}$ and $\Lambda_{\varphi}^{\psi}$. From the geometrical set-up in the compactification, we realize that $\Lambda_{\varphi}^{\psi e}$ may be viewed as an intersection "at infinity" of the two. This results in an additional property that these kind of submanifolds, arising from SG-classical phase functions, have, which may be viewed as a "conormality in the corner"-condition.

Lemma 3.32. The pairing $\langle x, \xi\rangle$ vanishes on $\Lambda_{\varphi}^{\psi e}$.

Proof. On $\Lambda_{\varphi}^{\psi e}$ we have, by Euler's theorem for homogeneous functions applied twice,

$$
\langle x, \xi\rangle=\left\langle x, \nabla_{x} \varphi^{\psi e}(x, \theta)\right\rangle=\varphi^{\psi e}(x, \theta)=\theta \cdot \nabla_{\theta} \varphi^{\psi e}(x, \theta)=0 .
$$

With the previous characterizations providing a motivation, we will now define SG-Lagrangians. A SG-Lagrangian $\tilde{\Lambda}$ is a collection of submanifolds of $\widetilde{\mathrm{W}}_{\mathrm{SG}}$ with Lagrangian properties. When defining this, we have to take into account that $\widetilde{\mathrm{W}}_{\mathrm{SG}}$ is not a manifold, but a pair of submanifolds of $\partial\left(\mathbb{B}^{d} \times \mathbb{B}^{d}\right)$ that intersect cleanly 
at their joint boundary, the corner of $\mathbb{B}^{d} \times \mathbb{B}^{d}$, see Figure 12 . Therefore, we have to consider a number of cases when we define such a pair of submanifolds. We first consider the case where $\tilde{\Lambda}$ intersects the corner.

Definition 3.33. A SG-Lagrangian $\widetilde{\Lambda}$ in $\widetilde{\mathrm{W}}_{\mathrm{SG}}$ with non-empty corner component is a pair of closed immersed submanifolds (with boundary) of $\mathbb{B}^{d} \times \mathbb{B}^{d}, \widetilde{\Lambda}=\left(\widetilde{\Lambda} e, \widetilde{\Lambda}^{\psi}\right)$, such that

- $\left(\widetilde{\Lambda}^{e}\right)^{o} \subset \widetilde{\mathrm{W}}_{\mathrm{SG}}^{e},\left(\widetilde{\Lambda}^{\psi}\right)^{o} \subset \widetilde{\mathrm{W}}_{\mathrm{SG}}^{\psi}$,

- $\operatorname{dim}\left(\widetilde{\Lambda}^{e}\right)=\operatorname{dim}\left(\widetilde{\Lambda}^{\psi}\right)=d-1$,

- $\left(\widetilde{\Lambda}^{e} \cap \widetilde{\Lambda}^{\psi}\right)=\partial \widetilde{\Lambda}^{e}=\partial \widetilde{\Lambda}^{\psi}=: \widetilde{\Lambda}^{\psi e} \subset \widetilde{\mathrm{W}}_{\mathrm{SG}}^{\psi e}$ (with $\left.\operatorname{dim}\left(\widetilde{\Lambda}^{\psi e}\right)=d-2\right)$ and the intersection being clean,

- on the associated conifications

$$
\Lambda^{e}:=\left(\Gamma \times \iota^{-1}\right)\left(\left(\widetilde{\Lambda}^{e}\right)^{o}\right), \Lambda^{\psi}:=\left(\iota^{-1} \times \Gamma\right)\left(\left(\widetilde{\Lambda}^{\psi}\right)^{o}\right), \Lambda^{\psi e}:=(\Gamma \times \Gamma)\left(\widetilde{\Lambda}^{\psi e}\right),
$$

we have

$$
\left.\alpha^{e}\right|_{\Lambda^{e}}=0,\left.\quad \alpha^{\psi}\right|_{\Lambda^{\psi}}=0,\left.\quad \alpha^{e}\right|_{\Lambda^{\psi e}}=\left.\alpha^{\psi}\right|_{\Lambda^{\psi}{ }^{e}}=0,
$$

- in canonical coordinates we have the conormality condition $\left.\langle x, \xi\rangle\right|_{\Lambda^{\psi_{e}}}=0$. The triple $\left(\Lambda^{e}, \Lambda^{\psi}, \Lambda^{\psi e}\right)$ is then called a conic SG-Lagrangian in $T^{*} \mathbb{R}^{d}$.

The "degenerate cases" are then straightforward to define. If there is no intersection in the corner, then one of the submanifolds (which will no longer have a boundary) may be empty, or they form two disjoint $d$-1-dimensional submanifolds of $\widetilde{\mathrm{W}}_{\mathrm{SG}}^{e}$ and $\widetilde{\mathrm{W}}_{\mathrm{SG}}^{\psi}$ respectively. We may then state, to sum up our previous characterizations of $\widetilde{\mathcal{C}}_{\varphi}$ and $\tilde{\Lambda}_{\varphi}$ as well as $\mathcal{C}_{\varphi}$ and $\Lambda_{\varphi}$ :

Theorem 3.34. Let $\varphi \in \mathrm{SG}_{\mathrm{cl}}^{1,1}\left(\mathbb{R}^{d} \times \mathbb{R}^{s}\right)$ non-degenerate. Then $\tilde{\Lambda}_{\varphi}=\left(\tilde{\Lambda}_{\varphi}^{e}, \tilde{\Lambda}_{\varphi}^{\psi}\right)$ is a SG-Lagrangian in $\widetilde{\mathrm{W}}_{\mathrm{SG}}$.

In analogy to Theorem 3.4, we may expect a converse result to hold locally, i.e. that every Lagrangian is locally parametrizable by some non-degenerate SG-phase function.

Before investigating into that direction, we note that the $\Lambda^{e}$-component of our Lagrangians are closely related to Legendrian submanifolds investigated - in the larger framework of scattering manifolds in lieu of $\mathbb{R}^{d}$ - by Melrose and Zworski [107], and in the semi-classical context in [70, 71] as mentioned in the introduction 
to this section.

Therein, the scattering one-form, that corresponds to $\alpha^{e}$, denoted by ${ }^{\mathrm{sc}} \alpha$ is defined on ${ }^{\text {sc }} T^{*} X$, which is an extension of the co-tangent bundle of a scattering manifold $X$ up to the boundary. The form ${ }^{\mathrm{sc}} \alpha$ is obtained by the contraction of $\omega$ with the "radial vector" - given in terms of the boundary defining function via $\tilde{x}^{2} \cdot \delta_{\tilde{x}}$ - and restriction to the fibers over the boundary of $X$, i.e. to ${ }^{\text {sc }} T_{\partial X}^{*} X$.

A Legendrian submanifold of the boundary is then a submanifold of the boundary on which ${ }^{\mathrm{sc}} \alpha$ vanishes. [107, 70, 71] proceed by indicating parametrization results and to study "intersecting Legendrians with conical points". The associated distributions, Legendrian distributions, are smooth functions in the interior and have oscillatory singularities at the boundary. In particular, on Euclidean spaces, the singularities of the Fourier transforms of such Legendrian distributions on Euclidean spaces are contained in compact sets, by [107, Prop. 10], a feature that is not true for our class of distributions.

To the best of our knowledge, this property of being "Lagrangian up to the corner" as in our setting, i.e. matching Lagrangian-type singularities both in the interior as well as at the boundary, is not covered in the literature so far, especially not in the formalism we use, which differs from the one in [107] by rather working, whenever possible, on the original manifold and conifications of its "ends" than on its compactification.

We now turn to a converse result to Theorem 3.34, which is the main result of [50] and guarantees that, given an SG-Lagrangian, one may always find a nondegenerate SG-phase function which locally parametrizes the Lagrangian. For its formulation, we observe that it is a straight forward adaptation to define (nondegenerate) SG-phase functions locally, that is on open subsets in $\mathbb{R}^{d}$.

Theorem 3.35. Let $\widetilde{\Lambda}=\left(\widetilde{\Lambda}^{e}, \widetilde{\Lambda}^{\psi}\right)$ be a SG-Lagrangian submanifold. Then $\widetilde{\Lambda}$ is locally parametrizable by a non-degenerate SG-classical phase function, that is, $\forall\left(y_{0}, \eta_{0}\right) \in \widetilde{\Lambda}$ there exist

(1) a neighbourhood $\tilde{U}$ of $\left(y_{0}, \eta_{0}\right)$ in $\mathbb{B}^{d} \times \mathbb{B}^{d}$,

(2) an open set $\tilde{V} \subset \mathbb{B}^{d} \times \mathbb{B}^{s}$,

(3) a function $\tilde{\varphi} \in \tilde{\gamma}^{-1} \tilde{y}^{-1} \mathscr{C}^{\infty}(\tilde{U})$ such that the corresponding (locally defined) phase function $\varphi=(\iota \times \iota)^{*}(\tilde{\varphi})$ is non-degenerate, 
such that these fulfil

$$
\widetilde{\Lambda} \cap \tilde{U}=\tilde{\lambda}_{\varphi}\left(\left\{\left(y_{0}, \gamma_{0}\right) \in \tilde{V} \cap \widetilde{\mathrm{B}}:\left(y_{0}, \gamma_{0}\right) \in \widetilde{\mathcal{C}}_{\varphi}\right\}\right) .
$$

Proof. We follow our proof as given in [49].

We will only consider the case where $\left(y_{0}, \eta_{0}\right) \in \widetilde{\Lambda}^{\psi e}$, since the other possible situations will be covered by the same argument. The underlying idea of the proof is classical, cf. [71] and [83, however we need to switch carefully between coordinates on the compactification $\tilde{\Lambda}_{\varphi}$ - where we may make use of continuity "up to the corner" - and such on the triple of conic manifolds $\left(\Lambda_{\varphi}^{e}, \Lambda_{\varphi}^{\psi}, \Lambda_{\varphi}^{\psi e}\right)$, where the symplectic structure is particularly accessible.

$\widetilde{\Lambda}^{\psi e}$ is a $(d-2)$-dimensional embedded submanifold of $\mathbb{S}^{d-1} \times \mathbb{S}^{d-1}$ and we may assume, in a neighbourhood $\tilde{U}$ of $\left(y_{0}, \eta_{0}\right)$, that $\widetilde{\Lambda}^{\psi e}$ may be locally parametrized (possibly after a rearrangement of variables) as

$$
\tilde{U} \cap \widetilde{\Lambda}^{\psi e}=\left\{y^{\prime}, y^{\prime \prime}, \sqrt{1-\left(y^{\prime}\right)^{2}-\left(y^{\prime \prime}\right)^{2}}, \sqrt{1-\left(\eta^{\prime}\right)^{2}-\left(\eta^{\prime \prime}\right)^{2}}, \eta^{\prime}, \eta^{\prime \prime}\right\} .
$$

Therein we have denoted independent variables by $\left(y^{\prime \prime}, \eta^{\prime}\right)$, defined for some $s \leq$ $d-1$ as $\eta^{\prime}=\left(\eta_{2}, \ldots, \eta_{s}\right)$ and $y^{\prime \prime}=\left(y_{s+1}, \ldots, y_{d-1}\right)$ and the remaining variables,

$$
\begin{aligned}
y^{\prime} & =\tilde{Y}^{\psi e}\left(y^{\prime \prime}, \eta^{\prime}\right), \\
\eta^{\prime \prime} & =\tilde{H}^{\psi e}\left(y^{\prime \prime}, \eta^{\prime}\right),
\end{aligned}
$$

are smoothly dependent on $\left(y^{\prime \prime}, \eta^{\prime}\right)$. We may further assume, in the chosen coordinate neighbourhood, that $y_{d}$ and $\eta_{1}$ are non-vanishing, that is $y_{d}>c$ and $\eta_{1}>c$ for some $1 \geq c>0$.

Since the intersection at the corner $\widetilde{\Lambda}^{\psi e}=\widetilde{\Lambda}^{e} \cap \widetilde{\Lambda}^{\psi}=\partial \widetilde{\Lambda}^{e}=\partial \widetilde{\Lambda}^{\psi}$ is clean, and we therefore have $T_{\widetilde{\Lambda}{ }_{e}} \widetilde{\Lambda}^{e} \cap T_{\widetilde{\Lambda}{ }_{e}} \widetilde{\Lambda}^{\psi}=T \widetilde{\Lambda}^{\psi e}$, we may find corresponding parametrizations of $\widetilde{\Lambda}^{e}$ and $\widetilde{\Lambda}^{\psi}$ near the corner point $\left(y_{0}, \eta_{0}\right)$ in terms of that of $\widetilde{\Lambda}^{\psi e}$, that is

$$
\begin{aligned}
& \tilde{U} \cap \widetilde{\Lambda}^{e}=\left\{y^{\prime}, y^{\prime \prime}, \sqrt{1-\left(y^{\prime}\right)^{2}-\left(y^{\prime \prime}\right)^{2}}, \eta_{1}, \eta^{\prime}, \eta^{\prime \prime}\right\}, \\
& \tilde{U} \cap \widetilde{\Lambda}^{\psi}=\left\{y^{\prime}, y^{\prime \prime}, y_{d}, \sqrt{1-\left(\eta^{\prime}\right)^{2}-\left(\eta^{\prime \prime}\right)^{2}}, \eta^{\prime}, \eta^{\prime \prime}\right\} .
\end{aligned}
$$

Here the independent coordinates are $\left(y^{\prime \prime}, \eta_{1}, \eta^{\prime}\right)$ on $\widetilde{\Lambda}^{e}$ and $\left(y^{\prime \prime}, y_{d}, \eta^{\prime}\right)$ on $\widetilde{\Lambda}^{\psi}$. The remaining variables on each face may be chosen as smooth functions up to the 
boundary, that is on $\tilde{U} \cap \widetilde{\Lambda}^{e}$ we may write

$$
y^{\prime}=\tilde{Y}^{e}\left(y^{\prime \prime}, \eta_{1}, \eta^{\prime}\right), \quad \eta^{\prime \prime}=\tilde{H}^{e}\left(y^{\prime \prime}, \eta_{1}, \eta^{\prime}\right)
$$

and on $\tilde{U} \cap \widetilde{\Lambda}^{\psi}$ as

$$
y^{\prime}=\tilde{Y}^{\psi}\left(y^{\prime \prime}, y_{d}, \eta^{\prime}\right), \quad \eta^{\prime \prime}=\tilde{H}^{\psi}\left(y^{\prime \prime}, y_{d}, \eta^{\prime}\right)
$$

By $\widetilde{\Lambda}^{e} \cap \widetilde{\Lambda}^{\psi}=\partial \widetilde{\Lambda}^{e}=\partial \widetilde{\Lambda}^{\psi}=\widetilde{\Lambda}^{\psi e}$ we may conclude in this set of coordinates that for elements of the corner, that is

$$
\left(\eta_{1}, \eta^{\prime}, \tilde{H}^{e}\left(y^{\prime \prime}, \eta_{1}, \eta^{\prime}\right)\right) \in \mathbb{S}^{d-1} \text { and }\left(\tilde{Y}^{\psi}\left(y^{\prime \prime}, y_{d}, \eta^{\prime}\right), y^{\prime \prime}, y_{d}\right) \in \mathbb{S}^{d-1}
$$

we have

$$
\begin{aligned}
& \tilde{Y}^{e}\left(y^{\prime \prime}, \eta_{1}, \eta^{\prime}\right)=\tilde{Y}^{\psi}\left(y^{\prime \prime}, y_{d}, \eta^{\prime}\right)=\tilde{Y}^{\psi e}\left(y^{\prime \prime}, \eta^{\prime}\right) \\
& \tilde{H}^{e}\left(y^{\prime \prime}, \eta_{1}, \eta^{\prime}\right)=\tilde{H}^{\psi}\left(y^{\prime \prime}, y_{d}, \eta^{\prime}\right)=\tilde{H}^{\psi e}\left(y^{\prime \prime}, \eta^{\prime}\right)
\end{aligned}
$$

This choice of coordinates yields induced coordinates on the associated conifications $\Lambda^{e}=\left(\Gamma \times \iota^{-1}\right)\left(\widetilde{\Lambda}^{e}\right)$ and $\Lambda^{\psi}=\left(\iota^{-1} \times \Gamma\right)\left(\widetilde{\Lambda}^{\psi}\right)$. To establish these, we may take, as independent variables on $\Lambda^{e}$,

$$
x^{\prime \prime}=\left(\mu y^{\prime \prime}, \mu \sqrt{1-\left(y^{\prime}\right)^{2}-\left(y^{\prime \prime}\right)^{2}}\right), \quad \xi^{\prime}=\iota^{-1}\left(\eta_{1}, \eta^{\prime}\right) .
$$

In particular, $x^{\prime \prime}$ may be defined implicitly in terms of the map

$$
\left(y^{\prime \prime}, \mu\right) \mapsto\left(\mu(\mathrm{id} \times \iota)^{*} \tilde{Y}^{e}\left(y^{\prime \prime}, \xi^{\prime}\right), \mu y^{\prime \prime}, \mu \sqrt{1-\left((\mathrm{id} \times \iota)^{*} \tilde{Y}^{e}\left(y^{\prime \prime}, \xi^{\prime}\right)\right)^{2}-\left(y^{\prime \prime}\right)^{2}}\right) .
$$

We obtain that $x^{\prime}=\mu(\operatorname{id} \times \iota)^{*} \tilde{Y}^{e}\left(y^{\prime \prime}, \xi^{\prime}\right)=: X^{e}\left(x^{\prime \prime}, \xi^{\prime}\right)$ is a smooth function of $x^{\prime \prime}$ and $\xi^{\prime}$ and polyhomogeneous in $\xi^{\prime}$, of maximal degree 0 . By $\left|\left(x^{\prime}, x^{\prime \prime}\right)\right|=\mu$ it is further 1-homogeneous in $x^{\prime \prime}$. Similarly we have that $\xi^{\prime \prime}=\iota^{-1}\left((\operatorname{id} \times \iota)^{*} \tilde{H}^{e}\left(y^{\prime \prime}, \xi^{\prime}\right)\right)=$ : $\Xi^{e}\left(x^{\prime \prime}, \xi^{\prime}\right)$ is 0-homogeneous in $x^{\prime \prime}$ and polyhomogeneous in $\xi^{\prime}$.

Consequently, in a suitable neighbourhood of $\left(x_{0}, \xi_{0}\right)=\left(\mathrm{id} \times \iota^{-1}\right)\left(y_{0}, \eta_{0}\right)$, we may write

$$
\Lambda^{e}=\left\{\left(X^{e}\left(x^{\prime \prime}, \xi^{\prime}\right), x^{\prime \prime} ; \xi^{\prime}, \Xi^{e}\left(x^{\prime \prime}, \xi^{\prime}\right)\right\}\right.
$$

In the same way we may write, in coordinates

$$
x^{\prime \prime}=\iota^{-1}\left(y^{\prime \prime}, y_{d}\right), \quad \xi^{\prime}=\left(\mu \eta_{1}, \mu \eta^{\prime}\right),
$$

that

$$
\Lambda^{\psi}=\left\{\left(X^{\psi}\left(x^{\prime \prime}, \xi^{\prime}\right), x^{\prime \prime} ; \xi^{\prime}, \Xi^{\psi}\left(x^{\prime \prime}, \xi^{\prime}\right)\right\}\right.
$$


We may now define phase functions in terms of the above coordinate functions, parametrizing these conic submanifolds in the given neighbourhoods. We will first introduce phase functions which are possibly not non-degenerate, and will later reduce the number of variables to establish non-degeneracy. We set, on suitable open sets,

$$
\begin{aligned}
\phi^{e}(x, \xi) & =\left\langle x^{\prime}, \xi^{\prime}\right\rangle+\left\langle x^{\prime \prime}, \Xi^{e}\left(x^{\prime \prime}, \xi^{\prime}\right)\right\rangle, \\
\phi^{\psi}(x, \xi) & =\left\langle x^{\prime}, \xi^{\prime}\right\rangle-\left\langle X^{\psi}\left(x^{\prime \prime}, \xi^{\prime}\right), \xi^{\prime}\right\rangle .
\end{aligned}
$$

From the above definitions of $\Xi^{e}$ and $X^{\psi}$ we may conclude that these are (poly-) homogeneous functions in the two sets of variables. In fact we observe that $\phi^{e}$ is 1-homogeneous in $x$ and 1-polyhomogeneous in $\xi$, whereas $\phi^{\psi}$ is 1-homogeneous in $\xi$ and polyhomogeneous in $x$, that is (i.e. $\phi^{e} \in \mathscr{P}_{e}^{1,1}$ and $\phi^{\psi} \in \mathscr{P}_{\psi}^{1,1}$ ). This may be observed by writing these functions, restricted to suitable neighbourhoods in $\mathbb{S}^{d-1} \times \mathbb{R}^{d}$ and $\mathbb{R}^{d} \times \mathbb{S}^{d-1}$ respectively, as

$$
\begin{aligned}
& \left.\phi^{e}(x, \xi)\right|_{\mathbb{S}^{d-1} \times \mathbb{R}^{d}}=(\mathrm{id} \times \iota)^{*} \underbrace{(\tilde{b})}_{\left.=:\left.\tilde{y} \cdot \tilde{\phi}^{e}\right|_{\widetilde{\mathrm{W}}_{\mathrm{S} G}^{e}}\left(\left\langle\left(y^{\prime}, y^{\prime \prime}, y_{d}\right), \iota^{-1}\left(\eta_{1}, \eta^{\prime}, \tilde{H}^{e}\left(y^{\prime \prime}, \eta_{1}, \eta^{\prime}\right)\right)\right)\right\rangle\right)} \\
& \left.\phi^{\psi}(x, \xi)\right|_{\mathbb{R}^{d} \times \mathbb{S}^{d-1}}=(\iota \times \mathrm{id})^{*} \underbrace{\left(\left\langle\iota^{-1}\left(y^{\prime}\right)-\iota^{-1}\left(\tilde{Y}^{\psi}\left(y^{\prime \prime}, y_{d}, \eta^{\prime}\right)\right),\left(\eta_{1}, \eta^{\prime}\right)\right\rangle\right)}_{=: \tilde{\eta}^{*} \cdot \tilde{\phi}^{\psi} \mid \widetilde{\mathbb{W}}_{\mathrm{SG}}^{e}} .
\end{aligned}
$$

Using $\iota^{-1}(y)=\frac{y}{|y|}(1-|y|)^{-1}=\tilde{y}^{-1} \frac{y}{|y|}$ for large arguments and Proposition $\left.1.20\right|^{49}$ we obtain the desired symbol properties.

We now show that $\phi^{e}$ and $\phi^{\psi}$ may be obtained as the respective principal symbol components of a single SG-phase function. For that we calculate the principal symbols of $\phi^{e}$ and $\phi^{\psi}$ by the means of the proof of Proposition 1.20 , that is by evaluation on the boundary. Using $\lim _{n \rightarrow \infty} \tilde{y_{n}} \iota^{-1}\left(y_{n}\right)=\frac{y}{|y|}$ in case $y_{n} \rightarrow y$ with $y_{n} \in\left(\mathbb{B}^{d}\right)^{o}$ and $y \in \mathbb{S}^{d-1}$ as well as (3.35) and (3.36) in (3.39) and (3.40) we obtain in the corner component

$$
\begin{aligned}
& \left.\sigma_{\psi}\left(\phi^{e}\right)\right|_{\mathbb{S}^{d-1} \times \mathbb{S}^{d-1}}=(\mathrm{id} \times \mathrm{id})^{*}\left\langle\left(y^{\prime}, y^{\prime \prime}, y_{d}\right),\left(\eta_{1}, \eta^{\prime}, \tilde{H}^{\psi e}\left(y^{\prime \prime}, \eta^{\prime}\right)\right)\right\rangle \\
& \left.\sigma_{e}\left(\phi^{\psi}\right)\right|_{\mathbb{S}^{d-1} \times \mathbb{S}^{d-1}}=(\mathrm{id} \times \mathrm{id})^{*}\left\langle y^{\prime}-\tilde{Y}^{\psi e}\left(y^{\prime \prime}, \eta^{\prime}\right),\left(\eta_{1}, \eta^{\prime}\right)\right\rangle .
\end{aligned}
$$

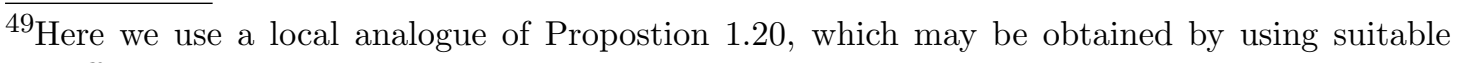
cut-offs.
} 
Consequently we have

$$
\begin{aligned}
\left.\sigma_{\psi}\left(\phi^{e}\right)\right|_{\mathbb{S}^{d-1} \times \mathbb{S}^{d-1}}- & \left.\sigma_{e}\left(\varphi^{\psi}\right)\right|_{\mathbb{S}^{d-1} \times \mathbb{S}^{d-1}}= \\
& (\mathrm{id} \times \mathrm{id})^{*}\left(\left\langle\tilde{Y}^{\psi e}\left(y^{\prime \prime}, \eta^{\prime}\right),\left(\eta_{1}, \eta^{\prime}\right)\right\rangle+\left\langle\left(y^{\prime \prime}, y_{d}\right), \tilde{H}^{\psi e}\left(y^{\prime \prime}, \eta^{\prime}\right)\right\rangle\right),
\end{aligned}
$$

which is nothing else than $\langle x, \xi\rangle$ at an element of $\Lambda^{\psi e}$ and thus vanishes by the conormality assumption. We are then able to, using (3.35) and (3.36) and Proposition 1.19 , continue $\left(\phi^{e}, \phi^{\psi}\right)$ to a single SG-symbol with principal symbol $\left(\phi^{e}, \phi^{\psi}, \phi^{\psi e}\right)$.

We note that, so far, this resulting phase function is by no means non-degenerate, since it is actually constant in the $\xi^{\prime \prime}$-variables. Getting rid of these redundant variables, we may define $\varphi: \mathbb{R}^{d} \times \mathbb{R}^{s} \rightarrow \mathbb{R}$ by $\left(\left(x^{\prime}, x^{\prime \prime}\right) ; \theta\right) \mapsto \phi\left(\left(x^{\prime}, x^{\prime \prime}\right) ;\left(\theta, \xi_{0}^{\prime \prime}\right)\right)$ for some arbitrary $\xi_{0}^{\prime \prime}$. We then obtain the components of the principal symbol $\varphi^{\bullet}=\sigma_{\bullet}(\varphi)$ for $\bullet \in\{e, \psi, \psi e\}$ and may define $\tilde{\varphi} \in \tilde{\gamma}^{-1} \tilde{y}^{-1} \mathscr{C}^{\infty}(\tilde{U})$ via $\left(\iota^{-1} \times \iota^{-1}\right)^{*} \varphi$. We now have to see that the functions $\varphi^{\bullet}$ indeed parametrize $\Lambda_{\varphi}$ and conclude non-degeneracy. From the vanishing of the one forms $\left.\alpha^{\bullet}\right|_{\Lambda} \bullet=0$ we obtain the identities

$$
\begin{array}{rlrl}
X^{e}\left(x^{\prime \prime}, \xi^{\prime}\right)+\nabla_{\xi^{\prime}}\left(x^{\prime \prime} \cdot \Xi^{e}\left(x^{\prime \prime}, \xi^{\prime}\right)\right) & =0, & \\
x^{\prime \prime} \cdot \partial_{x_{j}^{\prime \prime}} \Xi^{e}\left(x^{\prime \prime}, \xi^{\prime}\right)=0 & & j \in\{s+1, \ldots, d\}, \\
\theta \cdot \partial_{\xi_{k}^{\prime}} X^{\psi}\left(x^{\prime \prime}, \xi^{\prime}\right) & =0 & & k \in\{1, \ldots, s\}, \\
\nabla_{x^{\prime \prime}}\left(\theta \cdot X^{\psi}\left(x^{\prime \prime}, \xi^{\prime}\right)\right)+\Xi^{\psi}\left(x^{\prime \prime}, \xi^{\prime}\right) & =0 . &
\end{array}
$$

We may then compute, using (3.37) and (3.38),

$$
\begin{aligned}
& \nabla_{\theta} \varphi^{e}(x, \theta)=x^{\prime}+\underbrace{x^{\prime \prime} \cdot \nabla_{\theta} \Xi^{e}\left(x^{\prime \prime}, \theta\right)}_{=-X^{e}\left(x^{\prime \prime}, \theta\right)}, \\
& \partial_{\theta_{k}} \varphi^{\psi}(x, \theta)=\left(x_{k}^{\prime}-X_{k}^{\psi}\left(x^{\prime \prime}, \theta\right)\right)-\underbrace{\left(\partial_{\theta_{k}} X^{\psi}\left(x^{\prime \prime}, \theta\right)\right) \cdot \theta}_{=0} .
\end{aligned}
$$

and conclude that we have $\nabla_{\theta} \varphi^{\bullet}=0$ if and only if $x^{\prime}=X^{\bullet}\left(x^{\prime \prime}, \theta\right)$. We have obtained

$$
\mathcal{C}_{\varphi}^{\bullet}=\left\{\left(X^{\bullet}\left(x^{\prime \prime}, \theta\right), x^{\prime \prime} ; \theta\right)\right\}
$$


From this we may conclude non-degeneracy of $\varphi$. In a similar fashion, using the remaining two identities,

$$
\Lambda_{\varphi}^{\bullet}=\left\{\left(X^{\bullet}\left(x^{\prime \prime}, \theta\right), x^{\prime \prime} ; \theta, \Xi^{\bullet}\left(x^{\prime \prime}, \theta\right)\right)\right\}=\Lambda^{\bullet} .
$$

We can thus (locally) parametrize $\Lambda^{\bullet}$ by $\varphi^{\bullet}$. The proof is complete.

Having established that we can always find a (local) parametrizing phase function for such an SG-Lagrangian, we now state our result from [50] about when two such phase functions may be considered equivalent.

Theorem 3.36. Let $\tilde{\varphi}_{1}, \tilde{\varphi}_{2} \in \mathscr{C}^{\infty}\left(\mathbb{B}^{d} \times \mathbb{B}^{s}\right)$ be two non-degenerate phase functions that parametrize the same Lagrangian $\widetilde{\Lambda} \subset \mathrm{W}_{\mathrm{SG}}$ in a neighbourhood of some $\left(y_{0}, \eta_{0}\right) \in \widetilde{\Lambda}$. Assume that

(1) there exists $\left(y_{0}, \gamma_{0,1}\right) \in \widetilde{\mathcal{C}}_{\varphi_{1}}$ and $\left(y_{0}, \gamma_{0,2}\right) \in \widetilde{\mathcal{C}}_{\varphi_{2}}$ such that we have $\left(y_{0}, \eta_{0}\right)=$ $\tilde{\lambda}_{\varphi_{i}}\left(y_{0}, \gamma_{0, i}\right)$ and $\tilde{\varphi}_{1}\left(y_{0}, \gamma_{0,1}\right)=\tilde{\varphi}_{2}\left(y_{0}, \gamma_{0,2}\right), 50$

(2) The matrices $\left(\tilde{\gamma}^{-1} \tilde{y} \widehat{\left.\partial_{\theta_{j} \theta_{k}}^{2} \varphi_{1}\right|_{X}}\right)_{j, k=1, \ldots, s}$ and $\left(\left.\tilde{\gamma}^{-1} \tilde{y} \widetilde{\partial_{\theta_{j} \theta_{k}}^{2} \varphi_{2}}\right|_{X}\right)_{j, k=1, \ldots, s}$ have the same signature at $\left(y_{0}, \gamma_{0, i}\right) \in \widetilde{\mathcal{C}}_{\varphi_{i}}$, where $\varphi_{i}:=(\iota \times \iota)^{*} \tilde{\varphi}_{i}$ are the (locally defined) phase functions associated with $\tilde{\varphi}_{i}, i=1,2$.

Then, there exists a local homeomorphism $\tilde{\kappa}$ of the boundary $\widetilde{\mathrm{S}} \mapsto \widetilde{\mathrm{S}}$ that is defined in a neighbourhood of the $\left(y_{0}, \gamma_{0,2}\right)$ in the corresponding faces, which is smooth on each face and such that $\tilde{\varphi}_{2} \circ \tilde{\kappa}=\left.\tilde{\varphi}_{1}\right|_{\tilde{\mathrm{S}}}$.

Remark 3.37. The statement only ensures that the principal symbols $\left(\varphi_{i}^{e}, \varphi_{i}^{\psi}, \varphi_{i}^{\psi e}\right)$ of the corresponding phase functions $\varphi_{i}$ may be arranged to agree. This is, however, not a drawback, since the principal symbols of $\varphi_{i}$ carry all the information about the asscociated sets of singularities $\tilde{\Lambda}_{\varphi}$ and $\widetilde{\mathcal{C}}_{\varphi}$, by Lemmas 3.26 and 3.28 .

For a proof of Theorem 3.36, we refer to [50]. The idea of the proof is to adapt the classical outline of [79] and [55] to this global setting and in particular the corner. This is done by first arranging that the $\mathcal{C}_{\varphi_{i}}$ agree, and consequently the differentials of the involved phase functions (on principal level) locally agree up to first order. One then arranges, by use of a Taylor-like expansion and the implicit function theorem (and essentially Morse's Lemma) for the phase functions to be locally identical.

\footnotetext{
${ }^{50}$ We note that this is always fulfilled in the classical case since, by homogeneity, $\varphi_{i}$ vanishes on $\mathcal{C}_{\varphi_{i}}$.
} 


\subsection{Scaling properties of tempered distributions.}

\subsubsection{Weinstein's order and symbol of a (tempered) distribution.}

We have already seen in the context of the FBI-transform in Section 2.3.1 how scaling behaviour of distributions may be used to obtain microlocal information on their singularities. In this section, we will extend our findings by considering how the previous methods relate to the symbol construction of Weinstein [148]. In [148], Weinstein introduces a symbol for general distributions, which provides the means of classifying distributions "on principal level", that is up to "lower order terms", around a given points in phase-space $T^{*} X$. This symbol is introduced by studying the "magnitude of scaling" of a distribution at a given point when multiplied by a rapidly oscillating phase factor. The main result of [148] is that this notion of symbol coincides with the usual notion for Lagrangian distributions in case $u$ is a Lagrangian distribution with homogeneous principal symbol. The aim of this section is to show how Weinstein's definitions may be understood as part of the framework of phase-space analysis of distributions, especially considering our findings in Section 2.3.1, and to give an adapted definition of symbol that mimics the one which arises in the SG-calculus.

In [148, the order $N \in[-\infty, \infty)$ of a (a priori non-tempered) distribution $u \in$ $\mathscr{D}^{\prime}\left(\mathbb{R}^{d}\right)$ at a point in phase space $\left(0, \xi_{0}\right) \in T^{*} \mathbb{R}^{d} \backslash\{0\}$ is defined by saying that $u$ is of order $\leq N$ if the family $\left\{\lambda^{-N} u_{\varphi}^{\lambda} \mid \lambda \geq 1\right\}$ is bounded, where

- $\varphi$ is a real valued function $\varphi \in \mathscr{C}^{\infty}\left(\mathbb{R}^{d}\right), \varphi(0)=0$ and $d \varphi(0)=\xi_{0}$

- $u_{\varphi}^{\lambda}$ is the distribution $\left\langle u_{\varphi}^{\lambda}, f\right\rangle:=\left\langle u, \lambda^{d / 2} e^{-i \lambda \varphi(x)} f(\sqrt{\lambda} x)\right\rangle$.

Weinstein proceeds to investigate the dependence of the order on $\varphi$ ([148, Prop. 1.2.1]). He notices that if $\phi=\varphi+q+r$, where $q$ is a homogeneous quadratic polynomial and $r$ vanishes up to order at least 3 at 0 , then the order of $e^{i q} u_{\varphi}^{\lambda}-u_{\phi}^{\lambda}$ is $\leq N-1 / 2$.

It is then concluded ([148, Cor. 1.2.2]) that the order depends only upon $\varphi$ through its differential $d \varphi(0)$ and the factor $e^{i \varphi}$ may thus be replaced by modulation. We may thus reformulate using Proposition A.2 3) and [148, Lem. 1.1.4] (i.e. we may replace the lower bound $\lambda \geq 1$ by $\lambda \geq R$ for any $R$ ): 
Definition 3.38 (Weinstein's order of a distribution). We say $u \in \mathscr{S}^{\prime}\left(\mathbb{R}^{d}\right)$ is of order $\leq N$ at $\left(x_{0}, \xi_{0}\right)$ if for any window $g \in \mathscr{S}\left(\mathbb{R}^{d}\right)$

$$
\left\{\lambda^{-N+d / 4} \mathcal{D}_{\sqrt{\lambda}}\left\langle\mathcal{M}_{-\xi_{0}} \mathcal{T}_{-x_{0}} \mathcal{D}_{\sqrt{\lambda}^{-1}} u, g\right\rangle \mid \lambda>R\right\} \text { is bounded. }
$$

If the infimum $N_{0}:=\inf \{N \mid u$ is of order $\leq N\}$ is obtained, we say that the order of $u$ is $N_{0}$.

The condition (3.41) means nothing else than that the FBI transform of $u-$ with respect to an arbitrary window $g$ instead of $\psi_{0}-$ at $\left(x_{0},-\xi_{0}\right)$ is bounded by $\mathcal{O}\left(\lambda^{N-d / 4}\right)$, compare with A.6 and Proposition A.2 (3).

Remark 3.39. In light of the characterization of $\mathrm{WF}_{\mathrm{cl}}$ in terms of the FBI transform, that is Lemma 2.19, we have that [148, Corollary 2.2.2.] becomes evident, that is that the order of $u$ at $\left(x_{0}, \xi_{0}\right)=-\infty$ if $\left(x_{0}, \xi_{0}\right) \notin \mathrm{WF}_{\mathrm{cl}}(u)$, since the condition $\left(x_{0}, \xi_{0}\right) \notin \mathrm{WF}_{\mathrm{cl}}(u)$ just imposes the bound in (3.41) in an open neighbourhood of $\left(x_{0}, \xi_{0}\right)$.

The converse is not true, by [148, Example 2.2.4], which may be easily understood in the time frequency plane:

Weinstein constructs a distribution $u$ in $\mathbb{R}^{2}$ whose Fourier transform $\mathcal{F}_{(x, y) \rightarrow(\xi, \eta)} u$

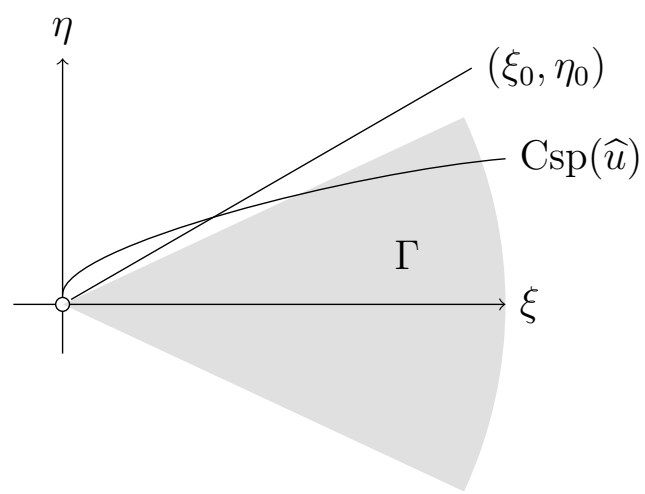

Figure 13. The counterexample of Weinstein, schematic

is singular on $\left\{\left(\xi, \xi^{\beta}\right) \mid \xi>0\right\}$, for $\beta \in(1 / 2,1)$. Of course this set of singularities stays in every cone $\Gamma$ around $(1,0)$, but is intersected at most once by any ray $\lambda\left(\xi_{0}, \eta_{0}\right)$, consider Figure 13 . He shows that the wave front set of $u$ at $(x, y)=0$ is non-empty, but the order there is $-\infty$ for all $\xi \neq 0$. 
The previous set-up allows for the definition of a symbol of a given distribution $u$. Weinstein originally takes the aforementioned behaviour under the change $\varphi \mapsto \varphi+q$ into account, we consider only the fixed phase $\varphi\left(x, \xi_{0}\right)=\left\langle x, \xi_{0}\right\rangle$. In this case, the symbol of order $N$ of a distribution which is of order $\leq N$ at $\left(x_{0}, \xi_{0}\right)$ is the ( $\lambda$-dependent) distribution

$$
g \mapsto \lambda^{d / 4} \mathcal{D}_{\sqrt{\lambda}}\left\langle\mathcal{M}_{-\xi_{0}} \mathcal{T}_{-x_{0}} \mathcal{D}_{\sqrt{\lambda}^{-1}} u, g\right\rangle
$$

modulo contributions of order $\leq N-1 / 2$. Weinstein then proceeds and calculates this symbol for Fourier integral distributions $u$ and proves that it coincides with the usual definition of principal symbol for Lagrangian distributions as introduced in [79], in case the principal symbol of $u$ is homogeneous.

For a generalization to tempered distributions, just as the SG calculus generalizes calculus of Hörmander symbols, one would assume that such a symbol construction must in the end yield three separate symbols, the standard one "at frequency infinity" $\sigma^{\psi}(u)$, one at "spacial infinity" $\sigma^{e}(u)$ and one at "both spacial and frequency infinity" $\sigma^{\psi e} u$.

Having noticed the connection of Weinstein's order and the FBI transform, we use the generalized FBI transform (compare with 2.7)

$$
g \mapsto \mathscr{F}_{\lambda, \mu}^{g}(u)\left(x_{0}, \xi_{0}\right):=\mu^{-3 d / 4} \lambda^{d / 4} \mathcal{D}_{\sqrt{\mu \lambda}}\left\langle\mathcal{M}_{-\xi_{0}} \mathcal{T}_{-x_{0}} \mathcal{D}_{\sqrt{\frac{\mu}{\lambda}}} u, g\right\rangle
$$

Definition 3.40 (SG-order of a distribution). Let $u \in \mathscr{S}^{\prime}\left(\mathbb{R}^{d}\right)$ and $\left(x_{0}, \xi_{0}\right) \in$ $\partial\left(\mathbb{B}^{d} \times \mathbb{B}^{d}\right)$. We say that $u$ has

(1) $\psi$-order $\leq m_{\psi} \in \mathbb{R}$ at $\left(x_{0}, \xi_{0}\right) \in \mathbb{R}^{d} \times \mathbb{S}^{d-1}$ if

$$
\left\{g \mapsto \lambda^{-m_{\psi}} \mathscr{F}_{\lambda, \mu}^{g}(u)\left(x_{0}, \xi_{0}\right) \mid \lambda>R, \mu=1\right\} \text { is bounded in } \mathscr{S}^{\prime}\left(\mathbb{R}^{d}\right)
$$

(2) $e$-order $\leq m_{e} \in \mathbb{R}$ at $\left(x_{0}, \xi_{0}\right) \in \mathbb{S}^{d-1} \times \mathbb{R}^{d}$ if

$$
\left\{g \mapsto \mu^{-m_{e}} \mathscr{F}_{\lambda, \mu}^{g}(u)\left(x_{0}, \xi_{0}\right) \mid \lambda=1, \mu>R\right\} \text { is bounded in } \mathscr{S}^{\prime}\left(\mathbb{R}^{d}\right)
$$

(3) $\psi e$-order $\leq\left(m_{e}, m_{\psi}\right) \in \mathbb{R} \times \mathbb{R}$ at $\left(x_{0}, \xi_{0}\right) \in \mathbb{S}^{d-1} \times \mathbb{S}^{d-1}$ if

$$
\left\{g \mapsto \lambda^{-m_{\psi}} \mu^{-m_{e}} \mathscr{F}_{\lambda, \mu}^{g}(u)\left(x_{0}, \xi_{0}\right) \mid \lambda>R, \mu>R\right\} \text { is bounded in } \mathscr{S}^{\prime}\left(\mathbb{R}^{d}\right)
$$

for some $R>0$. 
Using the continuity of $u: \mathscr{S}\left(\mathbb{R}^{d}\right) \rightarrow \mathbb{C}$ is is easy to prove that each of these orders are indeed bounded from above ${ }^{51}$ From the identity

$$
\mathscr{F}_{\lambda, \mu}^{g}(u)\left(x_{0}, \xi_{0}\right)=\lambda^{d} \mu^{-d} e^{i \mu \lambda x_{0} \xi_{0}} \mathscr{F}_{\mu, \lambda}^{\widehat{g}}(\widehat{u})\left(\xi_{0},-x_{0}\right)
$$

we conclude

Lemma 3.41. Let $u$ in $\mathscr{S}^{\prime}\left(\mathbb{R}^{d}\right)$. Then if

- $u$ is of $\psi$-order $\leq m_{\psi}$ at $\left(x_{0}, \xi_{0}\right) \in \mathbb{R}^{d} \times \mathbb{S}^{d-1}$, then $\widehat{u}$ is of e-order $\leq m_{\psi}-d$ at $\left(\xi_{0},-x_{0}\right)$.

- $u$ is of e-order $\leq m_{e}$ at $\left(x_{0}, \xi_{0}\right) \in \mathbb{S}^{d-1} \times \mathbb{R}^{d}$, then $\widehat{u}$ is of $\psi$-order $\leq m_{e}+d$ at $\left(\xi_{0},-x_{0}\right)$.

- $u$ is of $\psi$ e-order $\leq\left(m_{e}, m_{\psi}\right)$ at $\left(x_{0}, \xi_{0}\right) \in \mathbb{S}^{d-1} \times \mathbb{S}^{d-1}$, then $\widehat{u}$ is of $\psi$ e-order $\leq\left(m_{\psi}-d, m_{e}+d\right)$ at $\left(\xi_{0},-x_{0}\right)$.

For given $\left(m_{e}, m_{\psi}\right)$ we denote families (depending on the parameters $(\lambda, \mu)$ ) of distributions of the form

$$
\begin{aligned}
\mathrm{W}_{\mathrm{SG}} \times[1, \infty)^{2} & \rightarrow \mathscr{S}^{\prime}\left(\mathbb{R}^{d}\right) \\
(x, \xi, \lambda, \mu) & \mapsto\left(f \mapsto\left\langle u_{\lambda, \mu}(x, \xi), f\right\rangle\right)
\end{aligned}
$$

for which for some $R>0$

(1) $\forall\left(x_{0}, \xi_{0}\right) \in \mathbb{R}^{d} \times \mathbb{S}^{d-1}\left\{u_{\lambda, \mu}(x, \xi) \mid \lambda>R, \mu=1\right\}$ is bounded in $\mathscr{S}^{\prime}\left(\mathbb{R}^{d}\right)$

(2) $\forall\left(x_{0}, \xi_{0}\right) \in \mathbb{S}^{d-1} \times \mathbb{R}^{d}\left\{u_{\lambda, \mu}(x, \xi) \mid \lambda=1, \mu>R\right\}$ is bounded in $\mathscr{S}^{\prime}\left(\mathbb{R}^{d}\right)$

(3) $\forall\left(x_{0}, \xi_{0}\right) \in \mathbb{S}^{d-1} \times \mathbb{S}^{d-1}\left\{u_{\lambda, \mu}(x, \xi) \mid \lambda>R, \mu>R\right\}$ is bounded in $\mathscr{S}^{\prime}\left(\mathbb{R}^{d}\right)$

by by $\mathscr{S} \mathrm{G}^{\bullet}, m_{\psi}\left(\mathbb{R}^{d} \times \mathbb{R}^{d}\right) \mathscr{S} \mathrm{G}^{m_{e}, \bullet}\left(\mathbb{R}^{d} \times \mathbb{R}^{d}\right)$ and by $\mathscr{S} \mathrm{G}^{m_{e}, m_{\psi}}\left(\mathbb{R}^{d} \times \mathbb{R}^{d}\right)$ respectively. Given $u$ of $\psi$-order $\leq m_{\psi}, e$-order $\leq m_{e}$ and $\psi e$-order $\leq\left(m_{e}, m_{\psi}\right)$ at each possible $\left(x_{0}, \xi_{0}\right)$ we define the symbol $\sigma_{e}(u), \sigma_{\psi}(u)$ and $\sigma_{\psi e}(u)$ as the image of $\mathscr{F}_{\lambda, \mu}(u)$ in

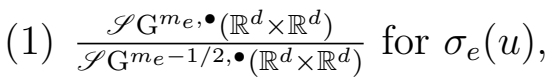

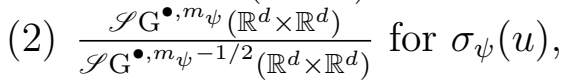

(3) $\frac{\mathscr{S} \mathrm{G}^{m_{e}, m_{\psi}}\left(\mathbb{R}^{d} \times \mathbb{R}^{d}\right)}{\mathscr{S} \mathrm{G}^{m_{e}-1 / 2, m_{\psi}}+\mathscr{S} \mathrm{G}^{m_{e}, m_{\psi}-1 / 2}\left(\mathbb{R}^{d} \times \mathbb{R}^{d}\right)}$ for $\sigma_{\psi e}(u)$.

This definition of symbol as a triple of equivalence classes then provides an analogue to Weinstein's notion in the SG-setting.

\footnotetext{
${ }^{51}$ For a proof using the same methods, consider that of the upcoming Lemma 3.44 .
} 


\subsubsection{The Steinmann scaling degree and extensions of distributions.}

The previous discussion was concerned with the order of a distribution at a given point $x_{0}$. For a finite point, Weinstein obtained it by scaling and testing with rapidly oscillating functions represented by $\xi_{0} \in \mathbb{S}^{d-1}$, thus picking up the the symbol, defined for $\left(x_{0}, \xi_{0}\right) \in \mathbb{R}^{d} \times \mathbb{S}^{d-1}$.

The upcoming theory may be understood as a similar study of the behaviour in the interior, i.e. at a given finite $\left(x_{0}, \xi_{0}\right) \in \mathbb{R}^{d} \times \mathbb{R}^{d}$. The scaling, however, makes any finite (or slowly oscillating) phase contribution "look constant" and thus negligible. We may therefore just consider $\xi_{0}=0$.

In the following, we thus wish to obtain information on $u$ at some point or lower dimensional subset $X \subset \mathbb{R}^{d}$ by scaling "towards $X$ ". Bounds on the scaling properties of $u$ - or asking for pointwise Hölder regularity - may be used to define, by magnification, "point values" and in more generality whole "Taylor expansions" of distributions, consider e.g. [17, 59, 96], ${ }^{52}$

The point of view we are going to take is that which arises in the continuation or extension problem, see [108, Chap. 1] as well as [16, Sect. 5] and [75, 112] which provides a framework for the problem of renormalization in perturbative quantum field theory.

In the following, we therefore assume that a distribution is "undefined at some $X \subset \mathbb{R}^{d "}$, meaning it is as a functional only defined for test functions $f$ with $X \cap \operatorname{supp}(f)=\emptyset$. We then wish to establish scaling criteria for $u$ to be extendible to the whole of $\mathbb{R}^{d}$.

Take first $x=0$. The solution to one such extension problem, the extension of singular integrals by the so-called method of finite parts, dates back to Hadamard. The main example for this procedure is the renormalization of $\mathbb{1}_{[0, \infty)} \frac{1}{x}$ : in order to make sense of the integral $\int_{0}^{\infty} \frac{f(x)}{x} d x$, which is defined for $f \in \mathscr{S}\left(\mathbb{R}^{d}\right)$ with $f(0)=0$, one replaces it for general $f$ by $\int_{0}^{M} \frac{f(x)-f(0)}{x}+\int_{M}^{\infty} \frac{f(x)}{x} d x$. This leaves us with the freedom to choose $M \in(0, \infty)$ and consequently two extensions of this form differ by $\ln \left(\frac{M_{2}}{M_{1}}\right) \delta_{0}$, see [117, Ex. V.3.9].

More generally, this continuation problem is well-understood for general homogeneous distributions, i.e. such that satisfy, for some $\kappa \in \mathbb{R},\left(\mathcal{D}_{\lambda}-\lambda^{\kappa+d / 2}\right) u=0$, see

\footnotetext{
${ }^{52}$ In 59, the connection $u(0)=\left\langle u, \delta_{0}\right\rangle$ is used to define the "adiabatic limit" $\langle u,, 1\rangle=$ $(2 \pi)^{-d}\left\langle\hat{u}, \delta_{0}\right\rangle$. In Sections 2.4.1 and 2.7.1 such pairings have already been studied from a microlocal point of view. In the following, we do not assume "microlocal rapid decay", but instead a scaling behaviour bounded by some power.
} 
[81, Sect. 3.2]. In this case, an extension is possible if the degree of homogeneity of the distribution satisfies $\kappa \leq-d$ and it is unique if $\kappa \notin \mathbb{Z}$. Otherwise, the extension is unique up to derivatives of $\delta_{0}$.

The idea in the following is to generalize this by assuming that $u$ is weakly-, or quasi-homogeneous, i.e. has a "leading scaling behaviour". This precisely leads to the notion of the Steinmann scaling degree, see [16, 127, 140] for the initial introduction and applications in quantum field theory.

Definition 3.42 (Steinmann scaling degree at the origin). Let $u \in \mathscr{S}^{\prime}\left(\mathbb{R}^{d}\right)$. The Steinmann scaling degree of $u$ at $x_{0}=0$ is

$$
\operatorname{sd}_{0}(u)=\inf \left\{\kappa \mid \lambda^{\kappa-d / 2} \mathcal{D}_{\lambda} u \stackrel{\lambda \rightarrow 0}{\longrightarrow} 0 \text { in } \mathscr{S}^{\prime}\left(\mathbb{R}^{d}\right)\right\}
$$

Instead of the origin, we can also consider extended surfaces up to dimension $d-1$. We will do so by considering the linear case 53 Throughout this section, we split $\mathbb{R}^{d} \cong \mathbb{R}^{d^{\prime}} \times \mathbb{R}^{k}$ with $d^{\prime}=d-k, k<d$, and denote the variables $\left(x^{\prime}, x^{\prime \prime}\right)$. Then we set $X=\{0\}^{d^{\prime}} \times \mathbb{R}^{k}$ and consider the "scaling transversal to $X$ " i.e. the transformation

$$
\mathcal{D}_{\lambda}^{X, \perp} f\left(x^{\prime}, x^{\prime \prime}\right):=\lambda^{\frac{d^{\prime}}{2}} f\left(\lambda x^{\prime}, x^{\prime \prime}\right)=\left(\mathcal{D}_{\lambda} \otimes \operatorname{id}_{\mathbb{R}^{k}}\right) f\left(x^{\prime}, x^{\prime \prime}\right) .
$$

Just as in Lemma A.6. we may extend $\mathcal{D}_{\lambda}^{X, \perp}$ to tempered distributions, by duality. We now define the scaling degree transversal to $X$.

Definition 3.43 (Transversal scaling degree). Let $u \in \mathscr{S}^{\prime}\left(\mathbb{R}^{d}\right)$. We say the transversal Steinmann scaling degree of $u$ at $X=\{0\}^{d^{\prime}} \times \mathbb{R}^{k}$ is

$$
\operatorname{sd}_{X}^{\perp}(u)=\inf \left\{\kappa \mid \lambda^{\kappa-d^{\prime} / 2} \mathcal{D}_{\lambda}^{X, \perp} u \stackrel{\lambda \rightarrow 0}{\longrightarrow} 0 \text { in } \mathscr{S}^{\prime}\left(\mathbb{R}^{d}\right)\right\}
$$

For any tempered distribution, this degree is always finite (or $-\infty)$ :

Lemma 3.44. Let $u \in \mathscr{S}^{\prime}\left(\mathbb{R}^{d}\right)$. Then there exist $\kappa \in \mathbb{R}$ such that $\operatorname{sd}_{X}^{\perp}(u) \leq \kappa$.

\footnotetext{
${ }^{53}$ Extensions of (non-tempered) distributions on manifolds, or "curved spacetimes", are discussed in [16, 112].
} 
Proof. From $u \in \mathscr{S}^{\prime}\left(\mathbb{R}^{d}\right)$ we gather that there exists some $(N, M) \in \mathbb{N}_{0}^{2}$

$$
\begin{aligned}
\left|\lambda^{-d^{\prime} / 2} \mathcal{D}_{\lambda}^{X, \perp} u, f\right\rangle \mid & =\lambda^{\kappa-d^{\prime} / 2}\left|\left\langle u, \mathcal{D}_{\lambda^{-1}}^{X, \perp} f\right\rangle\right| \\
& \lesssim \lambda^{-d^{\prime} / 2} \rho_{N, M}^{\mathscr{S}}\left(\mathcal{D}_{\lambda^{-1}}^{X, \perp} f\right) \\
& =\lambda^{-d^{\prime} / 2} \sup _{x \in \mathbb{R}^{d}} \sum_{|\alpha| \leq N} \sum_{|\beta| \leq M}\langle x\rangle^{|\alpha|}\left|\partial_{x}^{\beta} \mathcal{D}_{\lambda^{-1}}^{X, \perp} f\right| \\
& =\lambda^{-d^{\prime}} \sup _{x \in \mathbb{R}^{d}} \sum_{|\alpha| \leq N} \sum_{|\beta| \leq M}\left\langle\left(\lambda x^{\prime}, x^{\prime \prime}\right)\right\rangle^{|\alpha|}\left(\lambda, \mathbb{1}_{k}\right)^{-\beta}\left|\partial_{x}^{\beta} f\right|
\end{aligned}
$$

If we now assume $\lambda \leq 1$ we have

$$
\left|\left\langle\lambda^{-d^{\prime} / 2} \mathcal{D}_{\lambda}^{X, \perp} u, f\right\rangle\right| \lesssim \lambda^{-d^{\prime}-M} \rho_{N, M}^{\mathscr{S}}(f),
$$

from which we can gather that for $\kappa>d^{\prime}+M$ we have $\operatorname{sd}_{X}^{\perp}(u) \leq \kappa$.

Remark 3.45. We observe that we get a better control of the scaling degree if we use an equivalent family of semi-norms on $\mathscr{S}\left(\mathbb{R}^{d}\right)$ that is given by

$$
\rho_{N, M^{\prime}, M^{\prime \prime}}^{\mathscr{S} *}(f)=\sup _{x \in \mathbb{R}^{d}} \sum_{\substack{|\alpha| \leq N \\\left|\beta^{\prime}\right| \leq M^{\prime} \\\left|\beta^{\prime \prime}\right| \leq M^{\prime \prime}}}\langle x\rangle^{|\alpha|}\left|\partial_{x}^{\beta} f\right| \quad \beta=\left(\beta^{\prime}, \beta^{\prime \prime}\right) \in \mathbb{N}_{0}^{d^{\prime}} \times \mathbb{N}_{0}^{k} .
$$

Thus any tempered distribution (defined on the full space) has a finite scaling behaviour. The observation in [16, 108, 140] is that this notion may in turn be generalized to distributions that are a priori undefined on a subspace and used to extend these under the assumption that they admit a finite scaling behaviour.

Our main tool in the upcoming analysis will be a sequence of excision functions that approach $\mathbb{1}$ in a controlled manner, as in Construction 1.5, meaning a dyadic decomposition, cf. [108, Sect. 1.1]. We recall from this construction that there exists a positive function $\phi^{1} \in \mathscr{C}_{c}^{\infty}\left(\mathbb{R}^{d^{\prime}}\right)$ supported on $\left\{x \in \mathbb{R}^{d^{\prime}}|| x \mid \in[1 / 2,2]\right\}$ such that $\sum_{j=0}^{\infty} 2^{j d^{\prime} / 2} \mathcal{D}_{2^{-j}} \phi^{1}=\chi^{0}$, where $\chi^{0}$ is an excision function, supported in $\left\{x \in \mathbb{R}^{d^{\prime}}|| x \mid \in[1 / 2, \infty)\right\}$. In fact, we may express $\chi^{0}$ by the limit

$$
\chi^{0}=\mathbb{1}-\sum_{j=1}^{\infty} 2^{-j d^{\prime} / 2} \mathcal{D}_{2^{j}} \phi^{1}=: \mathbb{1}-\phi^{0} .
$$

Notation. In this Section, we always use the symbols $\phi^{0}, \phi^{1}$ and $\chi^{0}$ to denote the above, and not generic cut-offs and excision functions.

We now introduce the aforementioned tempered distributions that "are not defined on $X$ ". First we note that the definition of $\mathscr{D}\left(\mathbb{R}^{d} \backslash X\right)=\left\{f \in \mathscr{D}\left(\mathbb{R}^{d}\right) \mid \operatorname{supp}(f) \cap\right.$ 
$X=\emptyset\}$ and of the corresponding distribution space $\mathscr{D}^{\prime}\left(\mathbb{R}^{d} \backslash X\right)$ is canonical, see [81], since $\mathbb{R}^{d} \backslash X$ is an open subset of $\mathbb{R}^{d}$. For a space $\mathscr{S}^{\prime}\left(\mathbb{R}^{d} \backslash X\right)$ this is not the case, so we introduce it in detail.

Definition 3.46. We define the space of Schwartz functions on $\mathbb{R}^{d}$, vanishing of infinite order on $X$ as

$$
\mathscr{S}\left(\mathbb{R}^{d} \backslash X\right)=\left\{f \in \mathscr{S}\left(\mathbb{R}^{d}\right)\left|\partial^{\alpha} f\right|_{X} \equiv 0 \forall \alpha \in \mathbb{N}_{0}^{d}\right\},
$$

Before relating this to $\mathscr{D}\left(\mathbb{R}^{d} \backslash X\right)$, we study how we may decompose such functions "close to $X$ ". For that we first write an arbitrary $f \in \mathscr{S}\left(\mathbb{R}^{d}\right)$ as

$$
f(x)=f_{0}(x)+\sum_{|\alpha|=1}\left(x^{\prime}\right)^{\alpha} f_{\alpha ; 1}
$$

with functions $f_{0}, f_{\alpha ; 1} \in \mathscr{S}\left(\mathbb{R}^{d}\right)$ and $f_{0}\left(0, x^{\prime \prime}\right)=f\left(0, x^{\prime \prime}\right)$. We obtain this splitting by the following procedure:

1.) Set $f_{0}\left(x^{\prime}, x^{\prime \prime}\right)=f\left(0, x^{\prime \prime}\right) e^{-\frac{\left|x^{\prime}\right|^{2}}{2}}$.

2.) Observe that $f-f_{0} \in \mathscr{S}\left(\mathbb{R}^{d}\right)$, and it may be expressed as

$$
\left(f-f_{0}\right)\left(x^{\prime}, x^{\prime \prime}\right)=\int_{0}^{1} \frac{d}{d t}\left(f-f_{0}\right)\left(t x^{\prime}, x^{\prime \prime}\right) d t=x^{\prime} \cdot \int_{0}^{1}\left(\nabla_{x^{\prime}}\left(f-f_{0}\right)\right)\left(t x^{\prime}, x^{\prime \prime}\right) d t .
$$

Repeating this splitting on $f_{\alpha ; 1}$ we obtain, recursively, a splitting up to any degree $N \in \mathbb{N}_{0}$ (with $\alpha$ a multiindex in $\mathbb{N}_{0}^{d^{\prime}}$ )

$$
f=\sum_{|\alpha| \leq(N-1)}\left(x^{\prime}\right)^{\alpha} f_{\alpha}+\sum_{|\alpha|=N}\left(x^{\prime}\right)^{\alpha} f_{\alpha ; N} .
$$

where each $f_{\alpha}$ and $f_{\alpha ; N} \in \mathscr{S}\left(\mathbb{R}^{d}\right)$.

For $f \in \mathscr{S}\left(\mathbb{R}^{d} \backslash X\right)$ we calculate that $f_{0}=0$ and thus $\sum_{\alpha=1}\left(x^{\prime}\right)^{\alpha} f_{\alpha, 1}(x)=f \in$ $\mathscr{S}\left(\mathbb{R}^{d} \backslash X\right)$. By induction we gather $f=\sum_{|\alpha|=N}\left(x^{\prime}\right)^{\alpha} f_{\alpha ; N}$ for any finite $N$. We have obtained

Lemma 3.47. Let $f \in \mathscr{S}\left(\mathbb{R}^{d} \backslash X\right)$. Then for any $N \in \mathbb{N}_{0}$ we may decompose $f$ with respect to some $f_{\alpha} \in \mathscr{S}\left(\mathbb{R}^{d} \backslash X\right)$ as $f=\sum_{|\alpha|=N}\left(x^{\prime}\right)^{\alpha} f_{\alpha ; N}$.

We now recall that $\mathscr{D}\left(\mathbb{R}^{d}\right)$ is densely embedded in $\mathscr{S}\left(\mathbb{R}^{d}\right)$, and consequently $\mathscr{S}^{\prime}\left(\mathbb{R}^{d}\right)=\left\{u \in \mathscr{D}^{\prime}\left(\mathbb{R}^{d}\right) \mid u\right.$ is continuously extendible to $\left.\mathscr{S}\left(\mathbb{R}^{d}\right)\right\}$, meaning precisely that $|u(f)| \lesssim \rho_{N}^{\mathscr{S}}(f)$ for all $f \in \mathscr{D}\left(\mathbb{R}^{d}\right)$ and some $N \in \mathbb{N}_{0}$. For $\mathscr{S}\left(\mathbb{R}^{d} \backslash X\right)$ we are in a similar situation: 
Lemma 3.48. Let $f \in \mathscr{S}\left(\mathbb{R}^{d} \backslash X\right)$. Then, using the notation as above, we have that (with respect to the induced topology of $\mathscr{S}\left(\mathbb{R}^{d}\right)$ )

$$
\left(\chi^{0} \otimes \mathbb{1}_{\mathbb{R}^{k}}\right) f+\sum_{j=1}^{n}\left(\left(2^{-j d^{\prime} / 2}\left(\mathcal{D}_{2^{j}} \phi^{1}\right) \otimes \mathbb{1}_{\mathbb{R}^{k}}\right) f\right) \stackrel{n \rightarrow \infty}{\longrightarrow} f .
$$

Proof. Using an excision function and the support properties of $\chi^{0}$, we may replace w.l.o.g. $f$ by a function $g$ supported in the tube $Y_{n}$ around around $X$, where $Y_{n}=\left\{x \in \mathbb{R}^{d^{\prime}}|| x \mid \leq 2^{-n}\right\} \times \mathbb{R}^{k}$. We thus have $\left(\chi^{0} \otimes \mathbb{1}_{\mathbb{R}^{k}}\right) g=0$ and consequently we must verify that if $g \in \mathscr{S}\left(\mathbb{R}^{d} \backslash X\right)$ with support in $Y_{1}$, then

$$
g_{n}:=\sum_{j=1}^{n}\left(\left(2^{-j d^{\prime} / 2}\left(\mathcal{D}_{2^{j}} \phi^{1}\right) \otimes \mathbb{1}_{\mathbb{R}^{k}}\right) g\right) \stackrel{n \rightarrow \infty}{\longrightarrow} g
$$

It is easy to prove pointwise convergence, since by the support properties of $\phi^{1}$ only finitely many summands are non-zero at a given $x \notin X$ while $n \rightarrow \infty$. We now verify that the $g_{n}$ constitute a Cauchy sequence in $\mathscr{S}\left(\mathbb{R}^{d}\right)$. For that, we first verify for $m>n$

$$
\begin{aligned}
\rho_{N, M}^{\mathscr{S}}\left(g_{n}-g_{m}\right) & =\sup _{x \in \mathbb{R}^{d}} \sum_{|\alpha| \leq N} \sum_{|\beta| \leq M}\langle x\rangle^{|\alpha|}\left|\partial_{x}^{\beta}\left(g_{n}-g_{m}\right)\right| \\
& =\sup _{2^{-1-m} \leq\left|x^{\prime}\right| \leq 2^{-n}} \sum_{|\alpha| \leq N} \sum_{|\beta| \leq M}\langle x\rangle^{|\alpha|}\left|\partial_{x}^{\beta}\left(g_{m}\right)\right| .
\end{aligned}
$$

Using Lemma 3.47 and the support properties of the dilated $\phi^{1}$, we may bound this by an arbitrary high factor $\left|x^{\prime}\right|^{N}$. By $2^{-1-m} \leq\left|x^{\prime}\right| \leq 2^{-n}$ we see that the sequence is indeed a Cauchy sequence.

As a consequence of Lemma 3.48, we have the following Corollary:

Corollary 3.49. $\mathscr{D}\left(\mathbb{R}^{d} \backslash X\right)$ is densely embedded in $\mathscr{S}\left(\mathbb{R}^{d} \backslash X\right)$.

Consequently we may introduce the space $\mathscr{S}^{\prime}\left(\mathbb{R}^{d} \backslash X\right)$ as the space of such distributions in $\mathscr{D}^{\prime}\left(\mathbb{R}^{d} \backslash X\right)$ that are extendible to $\mathscr{S}\left(\mathbb{R}^{d} \backslash X\right)$. We may now introduce tempered distribution $\dot{u}$ on $\mathbb{R}^{d} \backslash X$ as an element $\dot{u} \in \mathscr{D}^{\prime}\left(\mathbb{R}^{d} \backslash X\right){ }^{54}$ which is extendible to all of $\mathscr{S}\left(\mathbb{R}^{d} \backslash X\right)$. The question of whether $\dot{u}$ is extendible to a tempered distribution on all of $\mathbb{R}^{d}$ breaks down into several parts

- Is there an extension $u \in \mathscr{D}^{\prime}\left(\mathbb{R}^{d}\right)$ ?

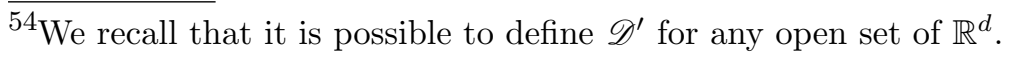


- Is it unique? If it is not, which degrees of freedom are there to extend $\dot{u}$ ?

- Can the extension be chosen/seen to be tempered, i.e. $u \in \mathscr{S}^{\prime}\left(\mathbb{R}^{d}\right)$ ?

We first generalize the notion of scaling degree to $\dot{u} \in \mathscr{S}^{\prime}\left(\mathbb{R}^{d} \backslash X\right)$.

Definition 3.50. Let $\dot{u} \in \mathscr{S}^{\prime}\left(\mathbb{R}^{d} \backslash X\right)$. We say $\operatorname{sd}_{X}^{\perp}(\dot{u}) \leq \kappa$ if $\forall f \in \mathscr{S}\left(\mathbb{R}^{d} \backslash X\right)$ we have $(N, M) \in \mathbb{N}_{0}^{2}$ such that for $\lambda \leq 1$ and

$$
\left|\left\langle\lambda^{\kappa-d^{\prime} / 2} \mathcal{D}_{\lambda}^{X, \perp} \dot{u}, f\right\rangle\right| \lesssim \rho_{N, M}^{\mathscr{S}}(f) .
$$

We may $\operatorname{set} \operatorname{sd}_{X}^{\perp}(\dot{u})=\inf \left\{\kappa \mid \operatorname{sd}_{X}^{\perp}(\dot{u}) \leq \kappa\right\}$.

We may obtain the following properties of this notion of scaling degree (compare with [16, Lem. 5.1]):

Lemma 3.51 (Properties of the scaling degree). Let $\dot{u} \in \mathscr{S}^{\prime}\left(\mathbb{R}^{d} \backslash X\right)$.

(1) For all $f \in \mathscr{S}\left(\mathbb{R}^{d}\right)$, we have $\operatorname{sd}_{X}^{\perp}(f \dot{u}) \leq \operatorname{sd}_{X}^{\perp}(\dot{u})$.

(2) Let $\dot{v} \in \mathscr{S}^{\prime}\left(\mathbb{R}^{d} \backslash X\right)$. Then $\operatorname{sd}_{X}^{\perp}(\dot{u}+\dot{v}) \leq \max \left\{\operatorname{sd}_{X}^{\perp}(\dot{u}), \operatorname{sd}_{X}^{\perp}(\dot{v})\right\}$

(3) Let $P \in \mathscr{C}^{\infty}\left(\mathbb{R}^{d^{\prime}}\right)$ a homogeneous polynomial of degree $N$. Then

$$
\operatorname{sd}_{X}^{\perp}\left(P\left(x^{\prime}\right) \dot{u}\right) \leq \operatorname{sd}_{X}^{\perp}(\dot{u})-N .
$$

(4) Let $P \in \mathscr{C}^{\infty}\left(\mathbb{R}^{d^{\prime}}\right)$ a homogeneous polynomial of degree $N$. Then

$$
\operatorname{sd}_{X}^{\perp}\left(P\left(\partial_{x^{\prime}}\right) \dot{u}\right) \leq \operatorname{sd}_{X}^{\perp}(\dot{u})+N .
$$

(5) Let $u$ of the form $u_{d^{\prime}} \otimes u_{k}$ with $u_{d^{\prime}} \in \mathscr{S}^{\prime}\left(\mathbb{R}^{d^{\prime}}\right)$ and $u_{k} \in \mathscr{S}^{\prime}\left(\mathbb{R}^{k}\right) \neq 0$. Then $\operatorname{sd}_{X}^{\perp}(u)$ only depends on $u^{d^{\prime}}$.

Proof. Property (1) may be obtained by the Banach-Steinhaus theorem for tempered distributions, that is Proposition A.2 (3). The other properties are straightforward applications of 3.42 .

Proposition 3.52. Let $\dot{u} \in \mathscr{S}^{\prime}\left(\mathbb{R}^{d} \backslash X\right)$ as above such that $\operatorname{sd}_{X}^{\perp}(\dot{u})=\kappa<\infty$. Then $\exists u \in \mathscr{S}^{\prime}\left(\mathbb{R}^{d}\right)$ such that

(1) $\left.u\right|_{\mathscr{S}\left(\mathbb{R}^{d} \backslash X\right)} \equiv \dot{u}$,

(2) $\operatorname{sd}_{X}^{\perp}(u) \leq \operatorname{sd}_{X}^{\perp}(\dot{u})$,

Furthermore we have 
(3) if $\kappa<d^{\prime}$, then $u$ is uniquely determined by the above conditions, else it is unique up to an element of

$$
\mathcal{R}_{\kappa-d^{\prime}}=\left\{\sum_{\left|\alpha^{\prime}\right| \leq \kappa-d^{\prime}} \partial_{x^{\prime}}^{\alpha^{\prime}} \delta_{X} \otimes v_{\alpha^{\prime}} \mid v_{\alpha^{\prime}} \in \mathscr{S}^{\prime}\left(\mathbb{R}^{k}\right)\right\} .
$$

Proof. The proof follows the outline in [16] and [112, with the additional aspects of taking temperedness into account. At the heart of the proof is the LittlewoodPaley-type partition of unity as introduced above 55

Suppose first that $\operatorname{sd}_{X}^{\perp}(\dot{u})<d^{\prime}$. Let $f \in \mathscr{S}\left(\mathbb{R}^{d}\right)$. We seek to define $\langle u, f\rangle$. Set (formally)

$$
\langle u, f\rangle:=\underbrace{\left\langle\left(\chi^{0} \otimes \mathbb{1}_{\mathbb{R}^{k}}\right) u, f\right\rangle}_{=:\left\langle u_{\chi}, f\right\rangle}+\underbrace{\left\langle\left(\phi^{0} \otimes \mathbb{1}_{\mathbb{R}^{k}}\right) u, f\right\rangle}_{=:\left\langle u_{\phi}, f\right\rangle}
$$

We give meaning to 3.43 term by term:

Since $\left(\chi^{0} \otimes \mathbb{1}_{\mathbb{R}^{k}}\right) f$ is an element of $\mathscr{S}\left(\mathbb{R}^{d} \backslash X\right)$, we can define the first term in accordance with assumption (1) to equal

$$
u_{\chi}:=\left(\chi^{0} \otimes \mathbb{1}_{\mathbb{R}^{k}}\right) \dot{u},
$$

trivially extended to an element of $\mathscr{S}^{\prime}\left(\mathbb{R}^{d}\right)$.

For the second term in (3.43), we use the decomposition of $\phi^{0}$ into the dilated copies of $\phi^{1}$. We first act as if $u_{\phi}$ was already extended, and do a formal calculation in order to motivate the upcoming definition of $u_{\phi}$ in terms of $\dot{u}$

$$
\begin{aligned}
\left\langle u_{\phi}, f\right\rangle & =\left\langle\left(\phi^{0} \otimes \mathbb{1}_{\mathbb{R}^{k}}\right) u, f\right\rangle \\
& =\left\langle u,\left(\phi^{0} \otimes \mathbb{1}_{\mathbb{R}^{k}}\right) f\right\rangle \\
& =\sum_{j=1}^{\infty} 2^{-j d^{\prime} / 2}\left\langle u,\left(\left(\mathcal{D}_{2^{j}} \phi^{1}\right) \otimes \mathbb{1}_{\mathbb{R}^{k}}\right) f\right\rangle \\
& =\sum_{j=1}^{\infty} 2^{-j d^{\prime} / 2}\left\langle\mathcal{D}_{2^{-j}}^{X, \perp} u, 2^{j d^{\prime} / 2}\left(\phi^{1} \otimes \mathbb{1}_{\mathbb{R}^{k}}\right) \mathcal{D}_{2^{-j}}^{X, \perp} f\right\rangle
\end{aligned}
$$

\footnotetext{
${ }^{55}$ Helpful comments on the role of boundedness and the Banach-Steinhaus theorem in the proof by Nguyen Viet Dang were gratefully received.
} 
Since $\left(\phi^{1} \otimes \mathbb{1}_{\mathbb{R}^{k}}\right) \mathcal{D}_{2^{-j}}^{X, \perp} f$ is an element of $\mathscr{S}\left(\mathbb{R}^{d} \backslash X\right)$, we can thus set, in accordance with assumption (1),

$$
\left\langle u_{\phi}, f\right\rangle:=\lim _{N \rightarrow \infty} \sum_{j=1}^{N} 2^{-j d^{\prime} / 2}\left\langle\mathcal{D}_{2^{-j}}^{X, \perp} u, 2^{j d^{\prime} / 2}\left(\phi^{1} \otimes \mathbb{1}_{\mathbb{R}^{k}}\right) \mathcal{D}_{2^{-j}}^{X, \perp} f\right\rangle .
$$

Each summand in (3.44) is well-defined. To guarantee the convergence of the sum, we want to use the bound on the scaling degree of $\dot{u}$ : since $\left(\phi^{0} \otimes \mathbb{1}_{\mathbb{R}^{k}}\right) \mathcal{D}_{2^{-j}}^{X, \perp} f$ is not a single test function, but actually a family of test functions with respect to the index $j$, to make use of 3.42 we need the following lemma, which may be obtained by similar estimates as those in the proof of Lemma 3.44 and 3.48 .

Lemma 3.53. The family of elements of $\mathscr{S}\left(\mathbb{R}^{d} \backslash X\right)$ given by

$$
\left\{f_{j}\right\}_{j \in \mathbb{N}_{0}}:=\left\{2^{j d^{\prime} / 2}\left(\phi^{1} \otimes \mathbb{1}_{\mathbb{R}^{k}}\right) \mathcal{D}_{2^{-j}}^{X, \perp} f\right\}_{j \in \mathbb{N}_{0}}
$$

is bounded in the topology of $\mathscr{S}\left(\mathbb{R}^{d}\right)$.

Using the version of the Banach-Steinhaus Theorem available for Schwartz functions, i.e. Proposition A.2 (3) we conclude by (3.42) that the family of distributions $2^{-j\left(\kappa-d^{\prime} / 2\right)} \mathcal{D}_{2^{-j}}^{X, \perp} \dot{u}$ is bounded on $\left\{f_{j}\right\}_{j \in \mathbb{N}_{0}}$, meaning that we have

$$
\begin{aligned}
\left\langle u_{\phi}, f\right\rangle & =\lim _{N \rightarrow \infty} \sum_{j=1}^{N} 2^{-j d^{\prime} / 2}\left\langle\mathcal{D}_{2^{-j}}^{X, \perp} u, f_{j}\right\rangle \\
& =\lim _{N \rightarrow \infty} \sum_{j=1}^{N} 2^{j\left(\kappa-d^{\prime}\right)}\left\langle 2^{-j\left(\kappa-d^{\prime} / 2\right)} \mathcal{D}_{2^{-j}}^{X, \perp} \dot{u}, f_{j}\right\rangle \\
& \lesssim \sum_{j=1}^{\infty} 2^{-j\left(d^{\prime}-\kappa\right)}<\infty \quad \text { by } \kappa<d^{\prime} .
\end{aligned}
$$

We obtain a well-defined element $u$ in $\mathscr{S}^{\prime}\left(\mathbb{R}^{d}\right)$, which satisfies $\left.u\right|_{\mathscr{S}\left(\mathbb{R}^{d} \backslash X\right)}=\dot{u}$, i.e. the claim (1), as a consequence of Lemma 3.48 .

Calculating $\left|\left\langle\lambda^{\kappa-d^{\prime} / 2} \mathcal{D}_{\lambda}^{X, \perp} u, f\right\rangle\right|$ by going through the construction once more, one obtains (2).

We postpone the question of uniqueness and first address the case $\infty>\operatorname{sd}_{X}^{\perp}(u) \geq$ $d^{\prime}$. We decompose $f$ as in the construction leading up to Lemma 3.47 and specify 
$N=\left\lfloor\operatorname{sd}_{X}^{\perp}(u)-d^{\prime}\right\rfloor+1$ and set

$$
\langle u, f\rangle:=\sum_{|\alpha|=N}\left\langle\left(\left(x^{\prime}\right)^{\alpha} \dot{u}\right)_{\text {ext. }}, f_{\alpha ; N}\right\rangle
$$

where the right-hand side is defined by the extension process granted by the first part of the proof, wherein we notice that, by Lemma 3.51, for $|\alpha|=N$, $\operatorname{sd}_{X}^{\perp}\left(\left(x^{\prime}\right)^{\alpha} \dot{u}\right) \leq d^{\prime}$

We have to make sure that this really extends $\dot{u}$, meaning that (1) is satisfied. For that we use the statement of Lemma 3.47, meaning we may write $f=\sum_{|\alpha|=N}\left(x^{\prime}\right)^{\alpha} f_{\alpha ; N}$, and compute, using that we have proved (1) for distributions with scaling degree less than $d^{\prime}$ in first part of the proof,

$$
\begin{aligned}
\langle u, f\rangle & =\sum_{|\alpha|=N}\left\langle\left(\left(x^{\prime}\right)^{\alpha} \dot{u}\right)_{\text {ext. }}, f_{\alpha ; N}\right\rangle \\
& =\sum_{|\alpha|=N}\left\langle\left(x^{\prime}\right)^{\alpha} \dot{u}, f_{\alpha ; N}\right\rangle \\
& =\left\langle\dot{u}, \sum_{|\alpha|=N}\left(x^{\prime}\right)^{\alpha} f_{\alpha ; N}\right\rangle \\
& =\langle\dot{u}, f\rangle .
\end{aligned}
$$

We now discuss the amount of possible extensions. By $\left.u\right|_{\mathscr{S}\left(\mathbb{R}^{d} \backslash X\right)} \equiv \dot{u}$, we have that the difference of two possible extensions $u-\tilde{u}=v \in \mathscr{S}^{\prime}\left(\mathbb{R}^{d}\right)$ must be supported on $X$. Recalling Theorem 2.52 , we can write such distributions as a finite sum of the form $v=\sum_{\left|\alpha^{\prime}\right| \leq N} \partial_{x^{\prime}}^{\alpha^{\prime}} \delta_{X} \otimes v_{\alpha^{\prime}}$, where $v_{\alpha^{\prime}} \in \mathscr{S}^{\prime}\left(\mathbb{R}^{k}\right)$. Calculating the scaling degree for such a $v$ is, by homogeneity of $\delta$, straight-forward and we obtain $\operatorname{sd}_{X}^{\perp}(v) \leq d^{\prime}+N$. To match assumption (2), we thus obtain (using Lemma 3.51(2)) that all possible extensions are obtained as the sum of one representative $u$ and an element of the subspace

$$
\mathcal{R}_{\kappa-d^{\prime}}=\left\{\sum_{\left|\alpha^{\prime}\right| \leq \kappa-d^{\prime}} \partial_{x^{\prime}}^{\alpha^{\prime}} \delta_{X} \otimes v_{\alpha^{\prime}} \mid v_{\alpha^{\prime}} \in \mathscr{S}^{\prime}\left(\mathbb{R}^{k}\right)\right\}
$$

Remark 3.54. We notice that when it comes to considering the extension problem, it is in fact unnecessary to consider the scaling degree of the whole distribution, since the construction is local. Since $\left(\chi^{0} \otimes \mathbb{1}_{\mathbb{R}^{k}}\right) \dot{u}$ is supported away from $X$, it 
was trivially extendible to all of $\mathscr{S}\left(\mathbb{R}^{d}\right)$ and we were left with the extension of $\left(\phi^{0} \otimes \mathbb{1}_{\mathbb{R}^{k}}\right) \dot{u}$. We may thus relax our condition on the scaling degree of $\dot{u}$ by imposing it only on $\operatorname{sd}_{X}^{\perp}\left(\left(\tilde{\phi}^{0} \otimes \mathbb{1}_{\mathbb{R}^{k}}\right) \dot{u}\right.$ ) for some cut-off $\tilde{\phi}^{0} \in \mathscr{C}^{\infty}\left(\mathbb{R}^{d^{\prime}}\right)$ (which is then necessarily smaller or equal to the original scaling degree, $\operatorname{sd}_{X}^{\perp}\left(\left(\tilde{\phi}^{0} \otimes \mathbb{1}_{\mathbb{R}^{k}}\right) \dot{u}\right) \leq \operatorname{sd}_{X}^{\perp}(\dot{u})$, by a Banach-Steinhaus type argument). We may localize even further by a partition of unity "along $X$ ". For the sake of comprehensibility, we have refrained from carrying out such straight-forward generalizations.

Remark 3.55. We remark that it is possible, under certain conditions, to estimate the wave front set of $\dot{u}$ in terms of $u$ which is a subset $T^{*} \mathbb{R}^{d} \backslash X$, see [16, Sect. 6] and [112, Thm. 3.2.1.]. Of course, we always have $\mathrm{WF}_{\mathrm{cl}}(u) \subset \mathrm{WF}_{\mathrm{cl}}(\dot{u}) \cup(X \times$ $\left.\left(\mathbb{R}^{d} \backslash\{0\}\right)\right)$. A particular case in which $\mathrm{WF}_{\mathrm{cl}}(u)$ may be bounded further is if the closure of $\Gamma=\overline{\mathrm{WF}_{\mathrm{cl}}(\dot{u})} \subset\left(\mathbb{R}^{d} \times \mathbb{R}^{d} \backslash\{0\}\right)$ stays in the conormal to $X$, and if the notion of scaling degree is refined with respect to the topology on the space $\mathscr{D}_{\Gamma}^{\prime}$ defined in [81, Sect. 8.2]

$$
\mathscr{D}_{\Gamma}^{\prime}=\left\{u \in \mathscr{D}^{\prime} \mid \mathrm{WF}_{\mathrm{cl}}(u) \subset \Gamma\right\},
$$

see [112, Sect. 3.1.1]. Therein, the resulting $u$ may be chosen to fulfil $\mathrm{WF}_{\mathrm{cl}}(u)=$ $\overline{\mathrm{WF}_{\mathrm{cl}}(\dot{u})}$.

It seems very likely that these considerations may be generalized to the tempered setting (with reference to the space $\mathscr{S}^{\prime}$ ), the details of which will be subject to further investigation.

We will now sketch how the previous theory may be applied to renormalize a priori undefined products of distributions. Let $u, v \in \mathscr{S}^{\prime}\left(\mathbb{R}^{d}\right)$. Assume that the assumptions of Proposition 2.42 hold outside a "subspace" $X \sqcup(X \backslash\{0\})$ of $\mathrm{WF}_{\mathrm{SG}}$. Then, for any excision function $\chi$ that vanishes on $X$, we have a well-defined product $(\chi u) \cdot v$. Suppose that by shrinking the support of $\chi$, we may extend this to a distribution $(u \cdot v)^{\cdot}$ on all $f \in \mathscr{S}^{X}\left(\mathbb{R}^{d}\right)$ and that $\operatorname{sd}_{X}^{\perp}\left((u \cdot v)^{\cdot}\right)<\infty .^{56}$

One may then define an extension of this product and call it the renormalized product of $u$ and $v$ - of course, if $\operatorname{sd}_{X}^{\perp}\left((u \cdot v)^{\cdot}\right) \geq d$, this product can only be regarded either as an equivalence class or it has to be determined by some choice of the ambiguity.

\footnotetext{
${ }^{56}$ To guarantee this, one would have to require that the convergence in the definition of the scaling degree (3.42) is "microlocal", as in Remark 3.55 that is impose convergence with respect to the notion of convergence of $\mathscr{S}_{\mathrm{WF}_{\mathrm{SG}}\left(u_{i}\right)}$.
} 
Remark 3.56. In quantum field theory, in one of the main applications of this theory, the remaining arising freedoms are essential and fixed by experimental data, see e.g. [127]. Note that this is - for $\operatorname{dim} X>0$ - in general only possible if we fix additional criteria, since in that case $\mathcal{R}_{\kappa-d^{\prime}}$ is not finite dimensional. The common way to do so is to reduce $\mathcal{R}_{\kappa-d^{\prime}}$ to a finite dimensional subspace by imposing $u$ to share certain symmetries of $\dot{u}$. Special cases of this could be

- $\dot{u}$ is (quasi-)homogeneous,

- $\dot{u}$ is translation invariant, or more generally invariant under some Lie group action,

- $\dot{u}$ is solves a differential equation.

It has been shown in [6], see also [152, Chap. 7] and [112, Chap. 8], how symmetries may be efficiently incorporated into the extension process.

The immediately arising question is if this renormalized product, when definable, yields an extension of the product of $\mathscr{S}$ in $\mathscr{S}^{\prime}$ in the previous sense of Section 2.1, i.e. an associative product that coincides with the usual one on continuous functions and which is subject to the Leibniz rule. The answer is no, since we can simply consider, in $\mathscr{S}^{\prime}(\mathbb{R})$, the example used in the proof of the impossibility result in [133] to see that on the one hand

$$
\left(x \cdot D^{2}(x(\log (|x|)-1)) \cdot \delta_{0}=\left(x \cdot \text { p.v. }\left(x^{-1}\right)\right) \cdot \delta_{0}=\mathbb{1} \cdot \delta_{0}=\delta_{0}\right.
$$

and on the other

$$
x \cdot\left(D^{2}\left(x(\log (|x|)-1) \cdot \delta_{0}\right)=x \cdot\left(\text { p.v. }\left(x^{-1}\right) \cdot \delta_{0}\right)=x \cdot(0) \stackrel{\text { ext. }}{\longrightarrow} x \cdot 0=0 .\right.
$$

In the same way we calculate $\delta_{0} \cdot \delta_{0}=(0) \stackrel{\text { ext. }}{\longrightarrow} 0$. One could of course, during the renormalization process, keep track of the scaling degrees of $u$ and $v$ and ask that the scaling degree of the resulting distribution matches that of the sum of that of $u$ and $v$, instead of that of $(u \cdot v)^{\cdot}$ which may be lower, yielding the freedom of setting $\delta_{0} \cdot \delta_{0}=C_{1} \delta_{0}+C_{2} \partial_{x} \delta_{0}$. Depending on the application under consideration, these ambiguities have to be fixed "manually" or the outcome of the product has to be viewed as an equivalence class, which leads into the area of algebras of generalized functions, see [25, 26, 27].

Remark 3.57. The methods previously used to extend distributions to subspaces can be generalized to yield extensions to submanifolds, see [16, 112]. 
We remark at this point that in light of the definition of the scaling degree, the methods used in extending to a submanifold $X$ can be used as well to extend distributions to tempered distributions and to such on bounded smooth functions. In fact, the usual method of proof to show that $\mathscr{D}\left(\mathbb{R}^{d}\right)$ is dense in $\mathscr{S}\left(\mathbb{R}^{d}\right)$ is by the use of an increasing sequence of cut-offs. The proof of Lemma 3.44 reveals how the finiteness of the scaling degree of a distribution stems from a finite order of a distribution - a scaling towards the boundary would be the same for the "multiplicative order" or "growth rate" of a tempered distribution.

Using a refined partition of unity as above and decomposing $\chi^{0}$ (instead of $\phi^{0}$ ) into factors of $\phi^{1}$, one could then carry out a similar analysis to the above. We have extended with respect to the spaces

$$
\mathscr{D}^{\prime}\left(\mathbb{R}^{d} \backslash X\right) \rightarrow \mathscr{S}^{\prime}\left(\mathbb{R}^{d} \backslash X\right) \rightarrow \mathscr{S}^{\prime}\left(\mathbb{R}^{d}\right),
$$

and accordingly one could extend distributions by

$$
\mathscr{D}^{\prime}\left(\mathbb{R}^{d}\right) \rightarrow \mathscr{S}^{\prime}\left(\mathbb{R}^{d}\right) \rightarrow\left(\mathscr{C}_{b}^{\infty}\right)^{\prime}\left(\mathbb{R}^{d}\right)
$$

by scaling "towards the boundary" instead of "towards $X$ ", see Figure 14 . The ambiguity in the extension process would amount to fixing the values of $\left\langle u,\langle x\rangle^{-n}\right\rangle$ for small values of $n \in \mathbb{N}_{0}$.

This procedure would then be applicable to problems in quantum field theory involving large scales, in particular the adiabatic limit, see Section 3.5.3.
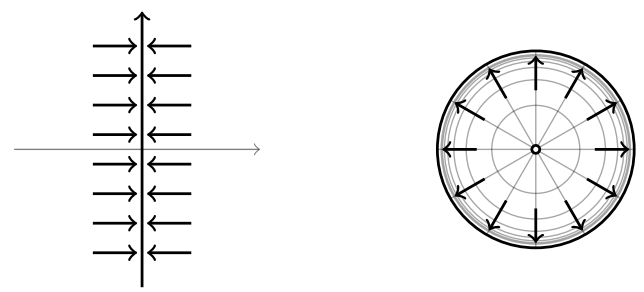

FIGURE 14. Schematic comparison between extensions by scaling 


\subsection{Weyl-quantized operators and the Weyl product.}

\subsubsection{Weyl products of tempered distributions.}

In this section, we address - in the spirit of Section 2.7.1- how the notion of $\mathrm{WF}_{\mathrm{G}}$ can be used to obtain existence criteria for twisted products, in particular the Weyl product, of tempered distributions and how the action of Weyl-quantized operators with distributional symbols can be extended to tempered distributions. Recall (see A.18) that the Weyl product of two symbols $a$ and $b$ takes the form

$$
a \# b(x, \xi)=\left.\exp \left(\frac{i}{2} \omega\left(D_{x}, D_{\xi} ; D_{y}, D_{\eta}\right)\right) a(x, \xi) b(y, \eta)\right|_{(x, \xi)=(y, \eta)}
$$

where the exponential is viewed as a Fourier multiplier. Using Theorem 2.83 and the symplectic transformation properties of the G-wave front set in Proposition 2.58, this can be extended to distributions:

Proposition 3.58. Let $\omega$ be a antisymmetric form on $\mathbb{R}^{d}$ given by the skewsymmetric matrix $\Theta$ via $\omega(x, y)=x^{t} \Theta y$ and let $u, v \in \mathscr{S}^{\prime}\left(\mathbb{R}^{d}\right)$.

If the equation

$$
x+\frac{\Theta}{2} \xi=0
$$

has no solutions with $(x, \xi) \in \mathrm{WF}_{\mathrm{G}}(u),(x,-\xi) \in \mathrm{WF}_{\mathrm{G}}(v)$, then we can define the twisted product in terms of the map $\delta(x)=(x, x)$ by

$$
u \#_{\Theta} v=\delta^{*} \mathcal{F}_{\left(x^{\prime}, y^{\prime}\right) \rightarrow(x, y)}^{-1}\left\{e^{\frac{i}{2}\left(x^{\prime}\right)^{t} \Theta y^{\prime}}(\mathcal{F} u)\left(x^{\prime}\right) \otimes(\mathcal{F} v)\left(y^{\prime}\right)\right\}
$$

in a canonical way and have the inclusion

$$
\begin{aligned}
\mathrm{WF}_{\mathrm{G}}\left(u \# \#_{\Theta} v\right) \subset \mathrm{WF}_{\mathrm{G}}(u) \cup \mathrm{WF}_{\mathrm{G}}(v) & \\
\cup & \left\{\left(\frac{x+y}{2}+\frac{\Theta}{2} \frac{\xi-\eta}{2}, \xi+\eta\right) \mid x-y=\frac{\Theta}{2}(\xi+\eta)\right\},
\end{aligned}
$$

where $(x, \xi) \in \mathrm{WF}_{\mathrm{G}}(u)$ and $(y, \eta) \in \mathrm{WF}_{\mathrm{G}}(v)$. This yields a sequentially continuous map $\#_{\Theta}: \mathscr{S}_{\mathrm{WF}_{\mathrm{G}}(u)}^{\prime} \times \mathscr{S}_{\mathrm{WF}_{\mathrm{G}}(v)}^{\prime} \rightarrow \mathscr{S}_{\Gamma}^{\prime}$ with $\Gamma$ given by the right-hand side in (3.47).

Remark 3.59. If $\mathbb{R}^{d}$ is even dimensional and $\omega$ is the standard symplectic form, then this twisted product yields the Weyl product.

In case $\Theta=0$, the twisted product degenerates to the usual product in case $\Theta=0$. The following proof therefore yields Proposition 2.86 (1) as a special case. 
Proof. We obtain (3.46) and 3.47) from Theorem 2.83 constructively by using Proposition 2.58 in several steps:

By the behaviour under tensor products, i.e. Lemma 2.84, and Fourier symmetry, we have

$$
\begin{aligned}
& \mathrm{WF}_{\mathrm{G}}(\mathcal{F} u\otimes \mathcal{F} v) \subset \\
& \quad\left\{(\xi, \eta,-x,-y) \mid(x, \xi) \in \mathrm{WF}_{\mathrm{G}}(u) \cup\{0\} ;(y, \eta) \in \mathrm{WF}_{\mathrm{G}}(v) \cup\{0\}\right\} \backslash\{0\} .
\end{aligned}
$$

By the skew-symmetry of $\Theta$ we may write

$$
e^{\frac{i}{2} x^{t} \Theta y}=e^{\frac{i}{2}(x, y)^{t} A(x, y)} \quad \text { for } \quad A=\left(\begin{array}{cc}
0 & \Theta / 2 \\
-\Theta / 2 & 0
\end{array}\right) .
$$

Notice that we have ${ }^{t} A=A$ and the symplectic transform corresponding to the unitary transform $f(x, y) \rightarrow e^{\frac{i}{2}(x, y)^{t} A(x, y)} f(x, y)$ is therefore

$$
\chi(x, y ; \xi, \eta)=\left(\begin{array}{cc}
\mathbb{1}_{\mathbb{R}^{2 d}} & 0 \\
A & \mathbb{1}_{\mathbb{R}^{2 d}}
\end{array}\right)(x, y ; \xi, \eta)=(x, y ; \xi+\Theta / 2 y, \eta-\Theta / 2 x) .
$$

Thus we have

$$
\begin{aligned}
& \mathrm{WF}_{\mathrm{G}}\left(\mathcal{F}^{-1}\left\{e^{\frac{i}{2} x^{t} \Theta y}(\mathcal{F} u \otimes \mathcal{F} v)\right\}\right) \subset \\
& \left\{\left(x-\frac{\Theta}{2} \eta, y+\frac{\Theta}{2} \xi, \xi, \eta\right) \mid(x, \xi) \in \mathrm{WF}_{\mathrm{G}}(u) \cup\{0\} ;(y, \eta) \in \mathrm{WF}_{\mathrm{G}}(v) \cup\{0\}\right\} \backslash\{0\} .
\end{aligned}
$$

The pullback $\delta^{*}$ corresponds to the linear map $x \mapsto(x, x)$ and thus its transpose is $(\xi, \eta) \mapsto \xi+\eta$. Therefore the pullback criterion 2.40 becomes

$$
\left(x-\frac{\Theta}{2} \eta, y+\frac{\Theta}{2} \xi\right)=(0,0) \Longrightarrow \xi+\eta \neq 0,
$$

which yields (3.46). We then use 2.41) to compute (3.47).

Remark 3.60. For nonsingular $\Theta$, the existence criterion may be reformulated as

$$
\mathrm{WF}_{\mathrm{G}}(u) \ni\left(x,-2 \Theta^{-1} x\right) \Rightarrow\left(x, 2 \Theta^{-1} x\right) \notin \mathrm{WF}_{\mathrm{G}}(v) .
$$

Remark 3.61. We note that the Weyl product is related to the so-called twisted convolution similarly to the way as the ordinary product is linked to the convolution, see [54, Sect. 3.6]. By the Fourier symmetry of $\mathrm{WF}_{\mathrm{G}}$, it is straightforward to extend our results about Weyl products to twisted convolutions. 
Corollary 3.62. Let $\Theta$ as in Proposition 3.58. Let $V$ be a conic subset of $\mathbb{R}^{d} \backslash\{0\}$ closed under addition and $W$ a conic subset of $\mathbb{R}^{d}$ such that

$$
x \in W, \xi \in V \Rightarrow x-\Theta \xi / 2 \in W .
$$

Define $\Gamma:=W \times V$. Then $\mathscr{S}_{\Gamma}^{\prime}\left(\mathbb{R}^{d}\right)$ is a topological algebra containing $\mathscr{S}\left(\mathbb{R}^{d}\right)$ which is closed under both products and twisted products.

Finding and studying such algebras was of interest - motivated by the so called "phase space approach to quantum mechanics" - in [66, 97, 54] and [137, 138, 139], cf. also the references therein for this wide subject.57 A similar train of thoughts is adapted in in [76, 144], where existence criteria for the Weyl product in weighted modulation spaces are indicated.

The main idea that all of these have in common is that they assume that the distributions in consideration fulfil global "growth estimates" and they proceed to estimate the Weyl product or twisted convolution by these. In this document, as discussed also in Section 2.1, we follow a microlocal approach to the question of existence of operations on distributions: we allow both factors to be rather wild in general (i.e. to be elements of $\mathscr{S}^{\prime}$ ), but impose that whenever one of the factors is not rapidly decaying in one direction in the time-frequency plane, i.e. microlocally, the other one is decaying in "a compensating direction". By our techniques - and by considering analogues of $\mathrm{WF}_{\mathrm{G}}$ adapted to the various norms and spaces used 58 . it seems very likely that our approach may be generalized further with respect to the spaces used in the aforementioned references.

\subsubsection{Weyl-quantization of tempered distributions.}

In this section we study how the previous results may be applied to Weyl-quantized operators and study their mapping properties. The question is natural, since Weyl products of distributions arise in particular if one considers products of pseudodifferential operators, where the amplitudes are allowed to be distributions, and one tries to set, for the composition,

$$
a^{W}(x, D) b^{W}(x, D)=(a \# b)^{W}(x, D) .
$$

\footnotetext{
${ }^{57}$ Note that in the latter sources, the algebras under consideration were tempered ultradistributions. These spaces can be treated similarly, but a modified version of the G-wave front set has to be used, see Section 4.1 .

${ }^{58}$ Such as Sobolev versions of $\mathrm{WF}_{\mathrm{G}}$.
} 
Weyl quantizations of distribution may of course be defined as in Appendix A.3. but it is unclear how to define compositions, since in general these provide operators $a: \mathscr{S}\left(\mathbb{R}^{d}\right) \rightarrow \mathscr{S}^{\prime}\left(\mathbb{R}^{d}\right)$. Using the machinery of Section 2.7, however, we may define such compositions if we can bound the G-wave front set of the involved kernels. Therefore, we express $a^{W}(x, D)$ as an operator with kernel $\mathcal{K}_{A}$, and compute $\mathcal{K}_{A}$ in several steps in terms of $a$ as follows (therein, we write distributions as formal integral kernels to be able to keep track of the variables):

(1) Take a distribution $a(x, \xi) \in \mathscr{S}^{\prime}\left(\mathbb{R}^{2 d}\right)$,

(2) tensor it with $\mathbb{1}$ to obtain $a(x, \xi) \mathbb{1}(y) \in \mathscr{S}^{\prime}\left(\mathbb{R}^{3 d}\right)$,

(3) apply a change of coordinates to obtain $A^{*}(a(x, \xi) \mathbb{1}(y))=a\left(\frac{x+y}{2}, \xi\right)$

(4) multiply by $e^{\frac{i}{2}(x, y, \xi)^{t} A(x, y, \xi)}=e^{i(x-y) \xi}$ with the real symmetric matrix

$$
A=\left(\begin{array}{ccc}
0 & 0 & \mathbb{1} \\
0 & 0 & -\mathbb{1} \\
\mathbb{1} & -\mathbb{1} & 0
\end{array}\right)
$$

to obtain $a\left(\frac{x+y}{2}, \xi\right) e^{i(x-y) \xi}$

(5) integrate over $\xi$, i.e. apply $\partial_{\xi^{*}=0} \mathcal{F}_{\xi \mapsto \xi^{*}}^{-1}$ to obtain

$$
\mathcal{K}_{A}(x, y)=(2 \pi)^{-d} \int_{\mathbb{R}^{d}} a\left(\frac{x+y}{2}, \xi\right) e^{i(x-y) \xi} d \xi \quad \in \mathscr{S}^{\prime}\left(\mathbb{R}^{2 d}\right) .
$$

Going through the individual steps and applying Proposition 2.58 and Theorem 2.83 , we see that the criterion for the pullback is always satisfied and using Proposition 2.88 we obtain

Proposition 3.63. Let $a \in \mathscr{S}^{\prime}\left(\mathbb{R}^{2 d}\right)$. Then the kernel $\mathcal{K}_{A}$ of the associated Weyl operator $A=a^{W}(x, D): \mathscr{S}\left(\mathbb{R}^{d}\right) \rightarrow \mathscr{S}^{\prime}\left(\mathbb{R}^{d}\right)$ satisfies

$$
\mathrm{WF}_{\mathrm{G}}\left(K_{A}\right) \subset\left\{\left(x-\frac{\xi^{*}}{2}, x+\frac{\xi^{*}}{2}, \frac{x^{*}}{2}+\xi, \frac{x^{*}}{2}-\xi\right) \mid\left(x, \xi, x^{*}, \xi^{*}\right) \in \mathrm{WF}_{\mathrm{G}}(a)\right\} .
$$

We may extend $A$ to $u \in \mathscr{S}^{\prime}\left(\mathbb{R}^{d}\right)$ with

$$
\mathrm{WF}_{\mathrm{G}}(u) \cap\left\{(x, \xi) \mid(x, \xi,-2 \xi, 2 x) \in \mathrm{WF}_{\mathrm{G}}(a)\right\}=\emptyset .
$$

We then have the inclusion

$$
\mathrm{WF}_{\mathrm{G}}(A u) \subset\left\{(x, \xi) \mid(x, \xi, 2 \xi, 2 x) \in \mathrm{WF}_{\mathrm{G}}(a)\right\} \cup \mathrm{WF}_{\mathrm{G}}^{\prime}\left(K_{A}\right) \circ \mathrm{WF}_{\mathrm{G}}(u) .
$$


In particular, for $f \in \mathscr{S}\left(\mathbb{R}^{d}\right)$ we have

$$
\mathrm{WF}_{\mathrm{G}}(A f) \subset \operatorname{pr}_{1}^{0}\left(\mathrm{WF}_{\mathrm{G}}^{\prime}\left(\mathcal{K}_{A}\right)\right)=\left\{(x, \xi) \mid(x, \xi, 2 \xi, 2 x) \in \mathrm{WF}_{\mathrm{G}}(a)\right\} .
$$

Remark 3.64. If $a \in \mathscr{S}^{\prime}\left(\mathbb{R}^{d}\right)$ happens to satisfy that all elements of $\mathrm{WF}_{\mathrm{G}}(u)$ are of the form $\{(x, \xi, 0,0)\}$, then $a^{W}(x, D)$ is $G$-microlocal, as is the case for e.g. elements of $G^{m}\left(\mathbb{R}^{d} \times \mathbb{R}^{d}\right)$.

Compositions of such Weyl operators with symbols $a, b \in \mathscr{S}^{\prime}\left(\mathbb{R}^{2 d}\right)$ are then defined if (3.48) is fulfilled, that is

$$
(x, \xi,-2 \xi, 2 x) \in \mathrm{WF}_{\mathrm{G}}(a) \Rightarrow(x, \xi, 2 \xi,-2 x) \notin \mathrm{WF}_{\mathrm{G}}(b) .
$$




\subsection{Applications to quantum field theory.}

\subsubsection{Introduction.}

Quantum field theory (QFT) is a theoretical framework to describe particle interactions at the (sub-)atomic level. Consequently, it describes high energy phenomena - and thus high frequencies - at small distances, which already hints at the applicability of microlocal methods to it.

There are various branches of quantum field theory which are active research topics, we are going to be concerned with axiomatic constructive quantum field theory, in particular constructions that arise in perturbation theory. We can not go into the details of these theories, which would be outside the scope of this dissertation, but merely focus on some applications of our findings to questions arising in QFT and provide the reader with some keywords about the context in which these constructions arise. Our main reference in the following discussion is [118].

One choice of minimal assumptions that a quantum field theory is supposed to fulfil is given by the Gårding-Wightman axioms, consider [118, Sect. IX.8] and the discussion therein. The fundamental objects of study in such quantum field theories are fields $\boldsymbol{\Phi}$. Such a field ${ }^{59}$ is mathematically modelled on some separable Hilbert space $\mathcal{H}$ as an operator-valued tempered distribution $\Phi: \mathscr{S}\left(\mathbb{R}^{d}\right) \rightarrow \operatorname{Op}(\mathcal{H})$, meaning $\forall v, w \in \mathcal{H}$ we have that $f \mapsto\langle v, \boldsymbol{\Phi}(f) w\rangle$ is a tempered distribution, for which one assumes, among other properties,

- the existence of some dense subset $\mathbf{D} \subset \mathcal{H}$ such that for any $f \in \mathscr{S}\left(\mathbb{R}^{d}\right)$ $\operatorname{dom}(\boldsymbol{\Phi}(f)) \supset \mathbf{D}$ and $\operatorname{dom}\left(\boldsymbol{\Phi}(f)^{*}\right) \supset \mathbf{D}$, and we have $\left.\boldsymbol{\Phi}(f)\right|_{\mathbf{D}}=\left.\boldsymbol{\Phi}^{*}(\bar{f})\right|_{\mathbf{D}}$,

- $\forall v \in \mathbf{D}$ the map $f \mapsto \boldsymbol{\Phi}(f) v$ is strongly continuous and linear,

- there exists a distinguished vector $\Omega \in \mathbf{D}$ called the vacuum.

The only theories (in $3+1$ dimensions) for which the full set of Gårding-Wightman axioms is known to hold, are the (generalized) free field theories (see [118, Sect. X.7]). These theories, however, are associated to linear partial differential equations and lack interaction terms. Interactions are then introduced into this framework in a perturbative approach. We consider here : $\boldsymbol{\Phi}^{n}$ :-theories, which may be viewed as toy models in QFT, but already capture a lot of the interesting structure.

In the Epstein-Glaser-approach to perturbation theory, one obtains the S-matrix

${ }^{59}$ Here, we assume our theory is described by only one type of field. 
to describe the transition from one state $v \in \mathcal{H}$ to another $w \in \mathcal{H}$ in an experiment with reference to Fermi's golden rule. This $S$-matrix is constructed as a formal power series (the Dyson's series) in the coupling constant $\lambda$

$$
S(g)=\mathbb{1}_{\mathcal{H}}+\sum_{n=1}^{\infty} \lambda^{n} \frac{(-i)^{n}}{n !}\left\langle\mathscr{T}_{n}, g^{\otimes n}\right\rangle \quad g \in \mathscr{S}\left(\mathbb{R}^{d}\right),
$$

where the $\mathscr{T}_{n}$ are some causal time-ordered products that are inductively constructed in terms of the free fields $\boldsymbol{\Phi}$.

The starting point of our analysis is that these time-ordered products may be expressed in terms of Wick products of fields and some formal integral kernels. Such expressions are then well-defined (tempered) operator valued distributions (by use of Epstein and Glaser's Theorem 0) if these formal integral kernels are (translation invariant) tempered distributions. It is therefore imperative to see that the arising expressions are indeed well-defined distributions - or may be extended to such.

We will analyse some of these expressions in the following. We mention that parts of this analysis has already been carried out in [129] and the two-point function has been already treated as an oscillatory integral in [49, 118]. Some of the upcoming constructions, in particular those involving the G-wave front set, are to the best of our knowledge entirely new. Others are generalizations of procedures that were established in terms of classical microlocal analysis, see [4], and we employ our methods to complement these with a study in the framework of tempered distributions.

Temperedness is of interest for several models of QFT, especially when one is also concerned with asymptotics of the involved distributions. It is of particular interest in the so-called non-commutative quantum field theory, meaning QFT over non-commutative space times, cf. e.g. [4, 5, 53], since in several of these models, the involved test functions can not be assumed compactly supported.

\subsubsection{The two-point function as a tempered oscillatory integral.}

In the following, we first consider free, scalar, bosonic QFT on flat space time, that is Minkowski space $\mathbb{M}^{d, 1}=\mathbb{R} \times \mathbb{R}^{d}$, wherein we denote points by $\left(x_{0}, x\right)$ for $x_{0} \in \mathbb{R}$ and $x=\left(x_{1}, \ldots, x_{d}\right) \in \mathbb{R}^{d}$. Elements of $\mathbb{M}^{1, d} \times \mathbb{R}^{d}$ are brought into the format $\left(x_{0}, x ; \theta\right)$.

Let $m>0$ the mass, $\omega_{m}(x)=\sqrt{m^{2}+|x|^{2}}$. The two-point function on $\mathbb{M}^{1, d} \times \mathbb{M}^{1, d}$ 
is obtained as (see [112, Chap. 5], [118, Sect. IX.8])

$$
\Delta_{+}\left(x_{0}-x_{0}^{\prime}, x-x^{\prime}\right):=i\left(\Omega, \boldsymbol{\Phi}\left(x_{0}, x\right) \boldsymbol{\Phi}\left(x_{0}^{\prime}, x^{\prime}\right) \Omega\right)_{\mathcal{H}}
$$

After passing to difference variables $\left(x_{0}-x_{0}^{\prime}, x-x^{\prime}\right) \mapsto\left(x_{0}, x\right)$ it may be represented by a formal integral expression on $\mathbb{M}^{1, d}$, that is

$$
\Delta_{+}\left(x_{0}, x\right):=\frac{i}{2(2 \pi)^{d}} \int_{\mathbb{R}^{d}} \frac{e^{i\left(-x_{0} \omega_{m}(\theta)+x \theta\right)}}{\omega_{m}(\theta)} d \theta .
$$

We observe that this is of the form of a formal oscillatory integral as in (3.1). We thus seek to define the two-point function as a tempered oscillatory integral and must verify that its symbol and phase function are SG-symbols. We note that we have already achieved such a characterization in [49]. Here we simplify the analysis by using that the SG-phase function of $\Delta_{+}$is classical.

For that, we observe that, by Example 1.11 , we have $\omega_{m}(\theta)=\sqrt{m^{2}+|\theta|^{2}}=$ $m\langle\theta / m\rangle \in \mathrm{S}_{\mathrm{cl}}^{1}\left(\mathbb{M}^{1, d}\right)$. Consequently we have

$$
\varphi\left(x_{0}, x ; \theta\right):=-x_{0} \omega_{m}(\theta)+x \theta \in \mathrm{SG}_{\mathrm{cl}}^{1,1}\left(\mathbb{M}^{1, d} \times \mathbb{R}^{d}\right)=\mathrm{S}^{1}\left(\mathbb{M}^{1, d}\right) \hat{\otimes} \mathrm{S}^{1}\left(\mathbb{R}^{d}\right) .
$$

We may compute the principal symbols and, by use of Proposition 1.18, their gradients at some $\left(x_{0}, x ; \theta\right)$ on their respective domains of definition

$$
\begin{aligned}
& \varphi^{\psi}=-x_{0}|\theta|+x \theta \quad \nabla_{\theta} \varphi^{\psi}=-x_{0} \frac{\theta}{|\theta|}+x \quad \nabla_{x} \varphi^{\psi}=(-|\theta|, \theta)^{t} \\
& \varphi^{e}=-x_{0} \omega_{m}(\theta)+x \theta \quad \nabla_{\theta} \varphi^{e}=-x_{0} \frac{\theta}{\omega_{m}(\theta)}+x \quad \nabla_{x} \varphi^{e}=\left(-\omega_{m}(\theta), \theta\right)^{t} \\
& \varphi^{\psi e}=-x_{0}|\theta|+x \theta \quad \nabla_{\theta} \varphi^{\psi e}=-x_{0} \frac{\theta}{|\theta|}+x \quad \nabla_{x} \varphi^{\psi e}=(-|\theta|, \theta)^{t}
\end{aligned}
$$

Since $\nabla_{x} \varphi^{\bullet}$ vanishes nowhere on $\mathrm{W}_{\mathrm{SG}}^{\bullet}$, the function $\Phi$ associated to $\varphi$ is $\mathrm{SG}$ elliptic, and $\varphi$ is indeed a classical $(1,1)$-SG-phase function. We may consequently use Theorem 3.9 to define $\Delta_{+}$:

Definition 3.65. The two-point function $\Delta_{+}$is the tempered oscillatory integral $\Delta_{+} \in \mathscr{S}^{\prime}\left(\mathbb{M}^{1, d}\right)$ with

$$
\begin{aligned}
& \varphi\left(x_{0}, x ; \theta\right)=-x_{0} \omega_{m}(\theta)+x \cdot \theta \\
& a\left(x_{0}, x ; \theta\right)=\frac{i}{2(2 \pi)^{d} \omega_{m}(\theta)},
\end{aligned}
$$

where $\left(x_{0}, x\right) \in \mathbb{M}^{1, d}$ and $\theta \in \mathbb{R}^{d}$.

Remark 3.66. This two-point function was already studied as an oscillatory integral in [118]. Therein, however, the phase function is "homogeneized" as in (3.8), that 
is by writing

$$
e^{i \varphi\left(x_{0}, x ; \theta\right)}=\underbrace{e^{i \varphi^{\psi}\left(x_{0}, x ; \theta\right)}}_{\text {Hom. phase }} \cdot(\underbrace{\chi(\theta) e^{i\left(\varphi-\varphi^{\psi}\right)\left(x_{0}, x ; \theta\right)}}_{\text {Cl. symbol }}+\underbrace{(1-\chi(\theta)) e^{i\left(\varphi-\varphi^{\psi}\right)\left(x_{0}, x ; \theta\right)}}_{\text {Compactly supported in } \theta}) .
$$

The draw-back of this procedure is that it introduces an artificial differential singularity in the symbol at $\theta=0$, which then needs to be treated as a asymptotic symbol. Such artificial singularities in $\theta=0$ influence the singularity structure of the resulting distribution at infinity, since the integration over a non-smooth perturbation - even if it is compactly supported - will not result in rapid decay. That means that this approach is not suited for the study of global singularities. We will see, in fact, that the mass parameter, which is removed from the phase, shows up in the e-component of the set of stationary points $\Lambda_{\varphi}^{e}$, and thus cannot be reproduced in the above approach if the mass term is absorbed into the symbol and "forgotten".

Our approach of instead treating $\Delta_{+}$as an oscillatory integral with inhomogeneous phase function is instead closely related to that of [156].

Another way to introduce the two-point function (see again [118]) is as the Fourier transform of some positive measure on the negative mass shell $H_{m}$

$$
\begin{aligned}
& H_{m}=\left\{\left(-\omega_{m}(\theta), \theta\right) \mid \theta \in \mathbb{R}^{d}\right\} \subset \mathbb{M}^{1, d} \\
& \Delta_{+}=i \pi \mathcal{F}_{\theta \mapsto x}^{-1} \frac{\delta_{H_{m}}}{\omega_{m}(\theta)}
\end{aligned}
$$

where $\delta_{H_{m}}$ is the distribution that acts on $f \in \mathscr{S}\left(\mathbb{M}^{1, d}\right)$ by

$$
\left\langle\delta_{H_{m}}, f\right\rangle=\int_{\mathbb{R}^{d}} f\left(-\omega_{m}(\theta), \theta\right) d \theta .
$$

From the principal symbols of $\varphi$, we may now establish bounds on the singularities of $\Delta_{+}$in terms of the associated geometric sets $\mathcal{C}_{\varphi}$ and $\Lambda_{\varphi}$. We calculate, by use of Lemma 3.26 ,

$$
\begin{aligned}
\mathcal{C}_{\varphi}^{\psi} & =\left\{(0,0 ; \theta) \mid \theta \in\left(\mathbb{R}^{d} \backslash\{0\}\right)\right\} \cup\left\{( \pm|x|, x ; \pm \lambda x) \mid x \in \mathbb{R}^{d} \backslash\{0\}, \lambda>0\right\} \\
\mathcal{C}_{\varphi}^{e} & =\left\{\left.\left( \pm x_{0}, x ; \frac{ \pm m x}{\sqrt{x_{0}^{2}-|x|^{2}}}\right)\left|x_{0} \in \mathbb{R}_{+}, x \in \mathbb{R}^{d},\right| x\right|^{2}<x_{0}^{2}\right\} \\
\mathcal{C}_{\varphi}^{\psi e} & =\left\{( \pm|x|, x ; \pm \lambda x) \mid x \in \mathbb{R}^{d} \backslash\{0\}, \lambda>0\right\}
\end{aligned}
$$


and $\Lambda_{\varphi}$ is, by Lemma and 3.28 , the union of 60

$$
\begin{aligned}
& \Lambda_{\varphi}^{\psi}=\left\{(0,0 ;-|\xi|, \xi) \mid \xi \in \mathbb{R}^{d}\right\} \cup\left\{( \pm|x|, x ;-\lambda|x|, \pm \lambda x) \mid x \in \mathbb{R}^{d} \backslash\{0\}, \lambda>0\right\} \\
& \Lambda_{\varphi}^{e}=\left\{\left.\left( \pm x_{0}, x ; \frac{-m\left|x_{0}\right|}{\sqrt{x_{0}^{2}-|x|^{2}}}, \frac{ \pm m x}{\sqrt{x_{0}^{2}-|x|^{2}}}\right)\left|x_{0} \in \mathbb{R}_{+}, x \in \mathbb{R}^{d},\right| x\right|^{2}<x_{0}^{2}\right\} \\
& \Lambda_{\varphi}^{\psi e}=\left\{( \pm|x|, x ;-\lambda|x|, \pm \lambda x) \mid x \in \mathbb{R}^{d} \backslash\{0\}, \lambda>0\right\}
\end{aligned}
$$

As in [49], we may parametrize the $e$-component of $\Lambda_{\varphi}$ also as follows:

$$
\Lambda_{\varphi}^{e}=\left\{\left( \pm \lambda \omega_{m}(\theta), \pm \lambda \theta ;-\omega_{m}(\theta), \theta\right) \mid \theta \in \mathbb{R}^{d}, \lambda>0\right\}
$$

Before we discuss the (physical) meaning of these sets, we first use Theorem 3.18 to calculate the singularities of $\Delta_{+}$, following our proof in [49, Thm 5.1].

Corollary 3.67. Let $\Delta_{+}, \mathcal{C}_{\varphi}$ and $\Lambda_{\varphi}$ as above, then

$$
\begin{aligned}
\operatorname{Css}\left(\Delta_{+}\right) & =\operatorname{pr}_{1}\left(\mathcal{C}_{\varphi}\right)=\operatorname{pr}_{1}\left(\Lambda_{\varphi}\right) \\
\mathrm{WF}_{\mathrm{SG}}\left(\Delta_{+}\right) & =\Lambda_{\varphi} .
\end{aligned}
$$

Proof. It is enough to prove the second statement. The inclusion $\subset$ follows by Theorem 3.18. The converse inclusion $\mathrm{WF}_{\mathrm{cl}}(u) \supset \Lambda^{\psi}$ was proven (by use of Lorentz invariance) in [118, Thm. IX.48]. The inclusion $\mathrm{WF}_{\mathrm{SG}}^{\psi e} \supset \Lambda^{\psi e}$ then follows by the closedness of $\mathrm{WF}_{\mathrm{SG}}$ in $\mathrm{W}_{\mathrm{SG}}$.

For the $\mathrm{WF}_{\mathrm{SG}}^{e}$-component, we make use of the fact that, see Lemma 2.4 ,

$$
(x, \xi) \in \mathrm{WF}_{\mathrm{SG}}^{e}\left(\Delta_{+}\right) \Leftrightarrow(\xi,-x) \in \mathrm{WF}_{\mathrm{SG}}^{\psi}\left(\mathcal{F} \Delta_{+}\right) .
$$

Making use of the explicit characterization (3.52) of $\mathcal{F} \Delta_{+}$and the parametrization (3.53) of $\Lambda_{\varphi}^{e}$ as well as the fact that the wave front set of this $\delta$-type distribution is well-known to be the set of normals to $H_{m}$ (see [81, Ex. 8.2.5]), we conclude the claim.

We now turn to a discussion of these sets. $\operatorname{pr}_{1}\left(\Lambda_{\varphi}^{\psi}\right)$ yields the light-cone, that is $\left\{\left(x_{0}, x\right)|| x_{0}|=| x \mid\right\}$, and $\left.\operatorname{pr}_{1}\left(\Lambda_{\varphi}^{\psi e}\right)\right)$ is simply the boundary of the light-cone "at infinity". That means all differential singularities of $\Delta_{+}$lie on the light cone. $\operatorname{pr}_{1}\left(\Lambda_{\varphi}^{e}\right)$ is formed by (all non-light-like) time-like directions, that is those that

\footnotetext{
${ }^{60}$ In the following, elements of $\mathbb{M}^{1, d} \times \mathbb{M}^{1, d}$ are brought into the format $\left(x_{0}, x ; \xi_{0}, \xi\right)$.
} 
satisfy $|x|^{2}<x_{0}^{2}$, and consequently $\Delta_{+}$is not rapidly decaying into these directions, but merely smooth and polynomially bounded. In the remaining space-like directions with $x_{0}^{2}<|x|^{2}$, it is smooth and of rapid decay. In $(1+1)$-dimensions, meaning $d=1$, this may be visualized as a subset of $\mathbb{B}^{d}$ in Figure 15 . In [118,

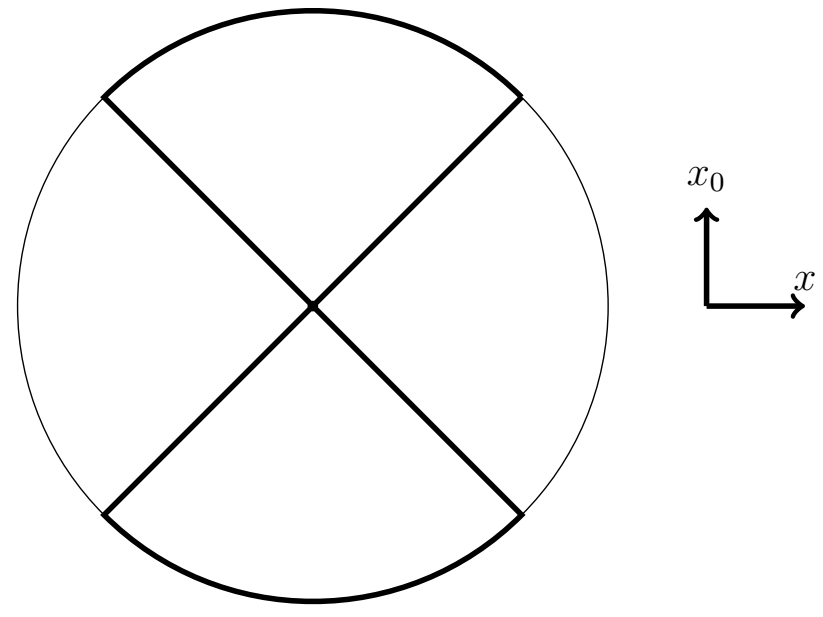

FiguRE 15. The cone singular support of the two-point function

Thm. IX.48], more precise decay rates for the two point function are given. We also note that the precise asymptotics of $\Delta_{+}$near the light-cone are known, see e.g. [11, App. II].

We now turn towards the set $\Lambda_{\varphi}$. The $\Lambda_{\varphi}^{\psi}$ and $\Lambda_{\varphi}^{\psi e}$-component are formed by attaching those tangential vectors to the light cone that have a negative $\xi_{0}$-component. $\Lambda_{\varphi}^{e}$ is best understood if one considers the set

$$
\mathrm{WF}_{\mathrm{SG}}^{e}\left(\mathcal{F} \Delta_{+}\right)=\left\{(\xi,-x) \mid(x, \xi) \in \mathrm{WF}_{\mathrm{SG}}^{\psi}\left(\Delta_{+}\right)\right\},
$$

as in the proof of Corollary 3.67, which is formed by the set of normals to $H_{m}$. This information (schematically, in $1+1$ dimensions) is visualized in Figure 16 , consider also [118, Fig. IX.7] and [50, Fig. 2].

Remark 3.68. We note that due to the conic singularity at $\left(x_{0}, x\right)=0, \mathcal{C}_{\varphi}^{\psi}$ is not a manifold, and thus $\varphi$ is not non-degenerate (around this point).

Having used our findings of Section 3.2 to define $\Delta_{+}$as a tempered distribution, we may now turn to the definition of further constructions involving $\Delta_{+}$. 

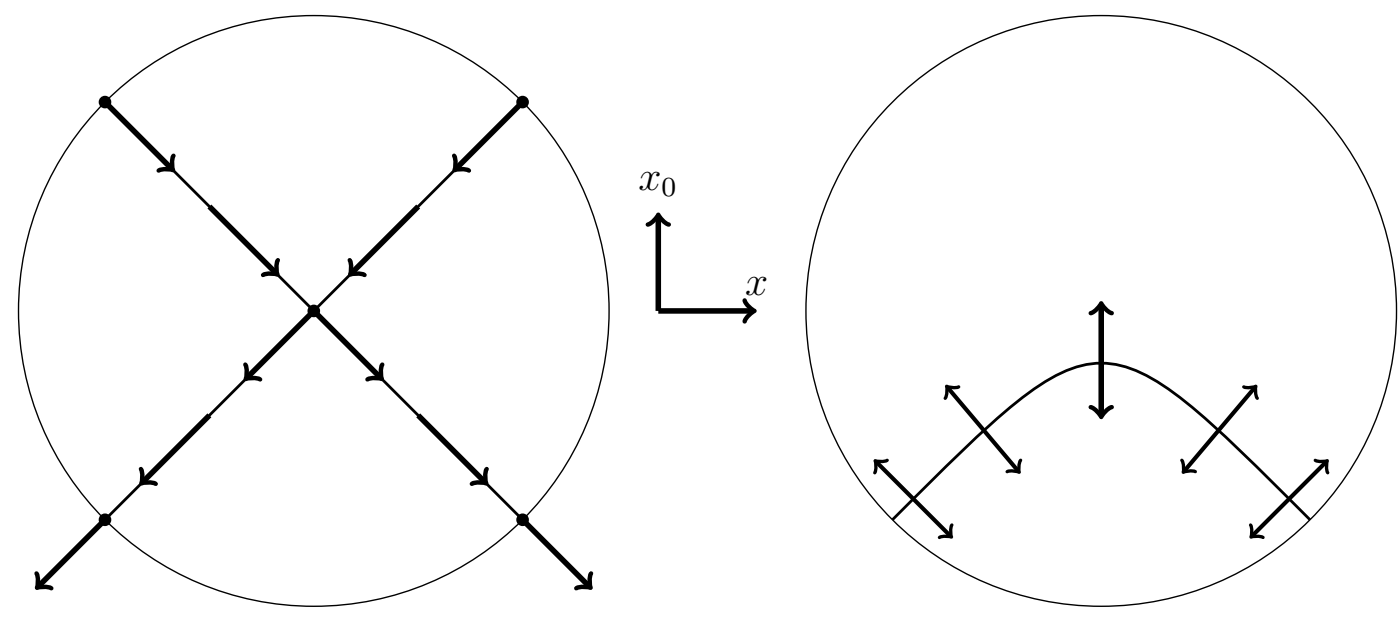

FigURE 16. $\widetilde{\mathrm{WF}}_{\mathrm{SG}}^{\psi}\left(\Delta_{+}\right) \cup \widetilde{\mathrm{WF}}_{\mathrm{SG}}^{\psi e}\left(\Delta_{+}\right)$and $\widetilde{\mathrm{WF}}_{\mathrm{SG}}^{\psi}\left(\mathcal{F} \Delta_{+}\right)$

\subsubsection{Well-definedness of distributional products in QFT.}

We have already mentioned time-ordered products in (3.49). In its simplest form, these occur in terms of the Heaviside-function $\vartheta=\mathbb{1}_{[0, \infty)} \in \mathscr{S}^{\prime}(\mathbb{R})$ by $(T$ denotes the time-ordering)

$$
\begin{aligned}
i(\Omega, & \left.T\left(\boldsymbol{\Phi}\left(x_{0}, x\right) \boldsymbol{\Phi}\left(x_{0}^{\prime}, x^{\prime}\right)\right) \Omega\right)_{\mathcal{H}} \\
\quad & i\left(\Omega, \vartheta\left(x_{0}-x_{0}^{\prime}\right) \boldsymbol{\Phi}\left(x_{0}, x\right) \boldsymbol{\Phi}\left(x_{0}^{\prime}, x^{\prime}\right) \Omega\right)_{\mathcal{H}}+\left(\Omega, \vartheta\left(x_{0}^{\prime}-x_{0}\right) \boldsymbol{\Phi}\left(x_{0}^{\prime}, x^{\prime}\right) \boldsymbol{\Phi}\left(x_{0}, x\right) \Omega\right)_{\mathcal{H}} \\
\quad & =\vartheta\left(x_{0}-x_{0}^{\prime}\right) \Delta_{+}\left(x_{0}-x_{0}^{\prime}, x-x^{\prime}\right)+\vartheta\left(x_{0}^{\prime}-x_{0}\right) \Delta_{+}\left(x_{0}^{\prime}-x_{0}, x^{\prime}-x\right)
\end{aligned}
$$

The latter expression constitutes the Feynman-Propagator, one of the distinguished fundamental solutions (see [56]) of the Klein-Gordon equation.

There are other ways of defining this distribution, in particular through a prescribed contour integration. We follow the train of thoughts of [118] of defining it as a product of distributions.

We therefore wish to define the product $\left(\vartheta \otimes \mathbb{1}_{\mathbb{R}^{d}}\right) \cdot \Delta_{+}$. For that, since we have already determined the wave front set of the two-point function, we first calculate by means of the analysis in Section 2.4.1

$$
\begin{aligned}
& \mathrm{WF}_{\mathrm{SG}}^{\psi}\left(\vartheta \otimes \mathbb{1}_{\mathbb{R}^{d}}\right)=\left\{(0, x, \pm \lambda, 0) \mid x \in \mathbb{R}^{d}, \lambda>0\right\} \\
& \mathrm{WF}_{\mathrm{SG}}^{\psi e}\left(\vartheta \otimes \mathbb{1}_{\mathbb{R}^{d}}\right)=\left\{(0, x, \pm \lambda, 0) \mid x \in\left(\mathbb{R}^{d} \backslash\{0\}\right), \lambda>0\right\} .
\end{aligned}
$$

Since $\mathrm{WF}_{\mathrm{SG}}^{\psi}\left(\Delta_{+}\right)$and $\mathrm{WF}_{\mathrm{SG}}^{\psi e}\left(\Delta_{+}\right)$contain no element of the form $(0, x, \pm \lambda, 0)$, we may conclude by Proposition 2.42 . 
Corollary 3.69. The product $\left(\vartheta \otimes \mathbb{1}_{\mathbb{R}^{d}}\right) \cdot \Delta_{+}$is a well-defined tempered distribution.

This statement was also established in [118] in the non-tempered setting.

In Dyson's series (3.49), several of factors of these Feynman propagators appear. Of course, these may be viewed as multiple products of Heaviside functions and two-point functions. These are, in general, not well-defined in terms of Proposition 2.42. Nevertheless, if one seeks a way to define such a product, it is necessary to first understand powers of the two-point function (since if $\Delta_{+}$was a function, we would for instance have $\left.\vartheta\left(x_{0}\right) \Delta_{+}\left(x_{0}, x\right) \vartheta\left(x_{0}\right) \Delta_{+}\left(x_{0}, x\right)=\vartheta\left(x_{0}\right) \Delta_{+}\left(x_{0}, x\right)^{2}\right)$. By Corollary 2.47, we conclude by induction

Corollary 3.70. For any $k \in \mathbb{N}_{0}$, the product $\Delta_{+}^{k}$ is a well-defined tempered distribution. Furthermore we have the wave front inclusion

$$
\begin{aligned}
& \mathrm{WF}_{\mathrm{SG}}^{\psi}\left(\Delta_{+}^{k}\right) \subset \mathrm{WF}_{\mathrm{SG}}^{\psi}\left(\Delta_{+}\right) \cup\left\{(0,0 ;-\lambda,|x|)|| x\left|\in \mathbb{R}^{d}, \lambda>\right| x \mid\right\} \\
& \mathrm{WF}_{\mathrm{SG}}^{e}\left(\Delta_{+}^{k}\right) \subset \\
& \qquad \bigcup_{j=1}^{k}\left\{\left.\left( \pm x_{0}, x ; \frac{-j m\left|x_{0}\right|}{\sqrt{x_{0}^{2}-x^{2}}}, \frac{ \pm j m x}{\sqrt{x_{0}^{2}-x^{2}}}\right)\left|\left(x_{0}, x\right) \in \mathbb{R}_{+} \times \mathbb{R}^{d},\right| x\right|^{2}<x_{0}^{2}\right\} \\
& \mathrm{WF}_{\mathrm{SG}}^{\psi e}\left(\Delta_{+}^{k}\right) \subset \mathrm{WF}_{\mathrm{SG}}^{\psi e}\left(\Delta_{+}\right)
\end{aligned}
$$

This information on the $\psi$ and the $\psi e$-components are visualized in Figure 17. We gather that the only newly arising problem to define $\vartheta \Delta_{+}^{k}$ is caused by highfrequency contributions at $\left(x_{0}, x\right)=0$. We may therefore still define $\vartheta \cdot\left(\chi \Delta_{+}^{k}\right)$ for any excision function $\chi$. The actual powers of the Feynman propagator may then only be obtained by renormalizing, that is extending, this product to zero by methods as in Section 3.3.2. This procedure, from a microlocal point of view, is carried out in [16, 112.

Remark 3.71. In this particular problem, we only have to extend the product to a single point, but for more complicated expressions, involving several variables, the products are ill-defined on whole subspaces, as in Section 3.3.2. In this setting, our methods may be used to control the asymptotic behaviour at infinity during the extension process, which may be of particular interest when studying the adiabatic limit, which is (termwise) obtained in the limit $g \rightarrow \mathbb{1}$ in (3.49). Of course, this 


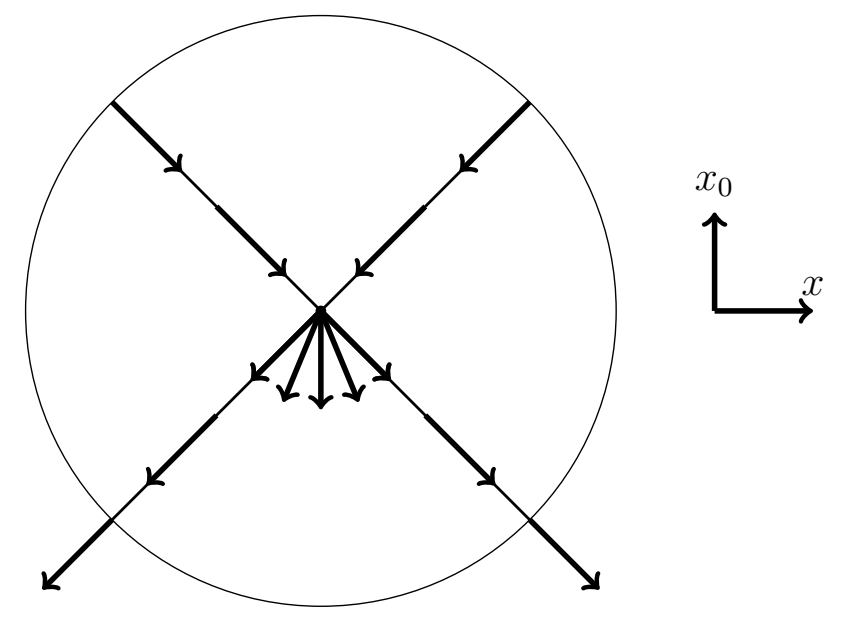

Figure 17. A bound for $\widetilde{\mathrm{WF}}_{\mathrm{SG}}^{\psi}\left(\Delta_{+}^{k}\right) \cup \widetilde{\mathrm{WF}}_{\mathrm{SG}}^{\psi e}\left(\Delta_{+}^{k}\right)$

limit is not in general well-defined, but could again be canonically obtained, see the discussion in Remark 3.55, by

- reducing the problem, by microlocal methods, to those points at infinity where the involved distributions are not rapidly decaying (for the Feynman propagator, this would be time-like points at infinity),

- applying sequences of cut-offs to extend the distributions to test functions of suitable decay,

- extending to all other bounded test functions, in particular $\mathbb{1}$ trivially and fixing the ambiguity by some convention (for instance by fixing accordance with some predicted experimental data).

Using our methods, it is thus possible to treat certain expressions that arise in QFT as tempered distributions. As previously mentioned, some models where asymptotic behaviour of distributions is of particular interest, come from the area of non-commutative quantum field theory. Again, we cannot introduce this area of study in great detail, and refer the reader to [4, 5, 53].

The idea behind these models is given by Doplicher, Fredenhagen and Roberts [53]: at small distances, that is at Planck-scale, if one interpolates elements from the relativistic theory of gravity, the nature of space-time must be fundamentally different from $\mathbb{M}^{1, d}$ in that distances cannot be measured with arbitrary precision. The arising bounds may be understood as uncertainty relations and may then be realized by replacing the space-time coordinates by non-commuting operators. 
Under these considerations, one may again formulate certain models, see [5, 53]. One feature which naturally occurs is that the point-wise product of functions (and distributions) is modified to accommodate the underlying non-commutativity. One of these models, where the product of fields is modified to a regularized product involving Gaussian integral kernels, which leads to a regularized quantum field theory, was treated from a microlocal point of view in [129]. Here, we will consider a different framework over the Minkowski Moyal plane, where the non-commutativity causes the presence of twisting factors.

Such deformations of the product are of course of great interest for the study of singularities. The hope is, that due to the "non-local nature" of non-commutative spaces ${ }^{61}$ singularities from models over corresponding "commutative space times" may be "smeared out", which might lead to models with less singularities. There occur, however, also new types of singularities, such as the famous infrared-ultraviolet mixing problem. These considerations have sprouted a whole field of study, we refer the reader to [4, 5] and the references therein.

The simplest example of one of the newly arising expressions that need to be defined as a distribution are twisted squares of the two-point function $\Delta_{+} \#_{\Theta} \Delta_{+}$for some anti-symmetric $\Theta$. We seek to define it by use of Proposition 3.58. For that, we establish bounds on the G-wave front set of $\Delta_{+}$.

By Proposition 2.78, we may deduce such bounds from the support and decay properties of $\mathcal{F} \Delta_{+}$and $\Delta_{+}$. By Corollary 3.67 , we see that $\Delta_{+}$is rapidly decaying in any direction $\left(x_{0} \infty, x \infty\right)$ with $\left|x_{0}\right|<|x|$. Additionally, any ray $\left(\xi_{0} \infty, \xi \infty\right)$ with $\xi_{0} \neq-|\xi|$ has a neighbourhood that does not intersect $\operatorname{Css}\left(\mathcal{F} \Delta_{+}\right)$, see Figure 16 . Therefore we may obtain the bound 62

$$
\mathrm{WF}_{\mathrm{G}}\left(\Delta_{+}\right) \subset\left\{\left(x_{0}, x ; \xi_{0}, \xi\right)|| x_{0}|\geq| x\left|, \quad \xi_{0}=-\right| \xi \mid\right\}
$$

We may thus, by Proposition 3.58, define the twisted square of $\Delta_{+}$, if 3.46 ) is fulfilled. By the above bound we have that $\left(x_{0}, x ; \xi_{0}, \xi\right) \in \mathrm{WF}_{\mathrm{G}}\left(\Delta_{+}\right)$and $\left(x_{0}, x ;-\xi_{0},-\xi\right) \in \mathrm{WF}_{\mathrm{G}}(u)$ implies $\left(\xi_{0}, \xi\right)=0$. Consequently, (3.46) is always fulfilled, and therefore we may define twisted squares for any skew-symmetric $\Theta$. By (3.47), the bound $-\xi_{0} \geq|\xi|$ is preserved under the twisted product. Consequently, we may even define any twisted power of $\Delta_{+}$. By Proposition 2.78, we

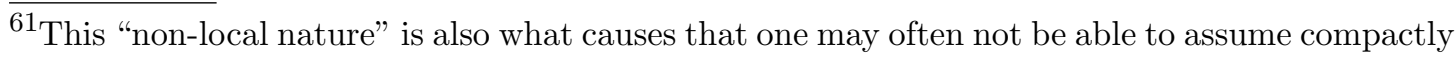
supported test functions.

${ }^{62}$ We may obtain this bound as well by considering the STFT of $\Delta_{+}$.
} 
may then obtain the bound $\left(x_{0}, x ; \xi_{0}, \xi\right) \in \mathrm{WF}_{\mathrm{cl}}(u) \Rightarrow-\xi_{0} \geq|\xi|$, that means that also for the twisted powers, the only "singular frequency directions" that occur are such in the interior of the negative-directed light-cone. This reproduces what we have encountered for the "ordinary" powers of $\Delta_{+}^{k}$, see Figure 17 . We have obtained

Proposition 3.72. For any $k \in \mathbb{N}, \Theta$ skew-symmetric, we may define the twisted power $\Delta_{+}^{\# k}$. We furthermore have the inclusions

$$
\begin{aligned}
& \left(x_{0}, x ; \xi_{0}, \xi\right) \in \mathrm{WF}_{\mathrm{G}}\left(\Delta_{+}^{\# k}\right) \Rightarrow-\xi_{0} \geq|\xi| \\
& \left(x_{0}, x ; \xi_{0}, \xi\right) \in \mathrm{WF}_{\mathrm{cl}}\left(\Delta_{+}^{\# k}\right) \Rightarrow-\xi_{0} \geq|\xi| .
\end{aligned}
$$

In particular, we may use Corollary 3.62 to obatin an algebra under both products as well as twisted products generated by $\Delta_{+}$and elements of $\mathscr{S}\left(\mathbb{R}^{d}\right)$. 


\section{Outlook}

In this thesis we have addressed how concepts from classical microlocal analysis may be translated and generalized to the global setting of tempered distributions. We were able to establish such generalizations for several constructions, and we have addressed multiple ways of how to characterize and work with global singularities. Nevertheless, there are still a lot of interesting directions into which our analysis may be pursued further. Before giving a general outlook on many topics of this thesis, we will first address how our methods may be transferred to a different functional setting, the Gelfand-Shilov spaces of funtions and ultradistributions.

\subsection{Microlocal analysis on Gelfand-Shilov spaces.}

In the previous analysis, we have established microlocal constructions involving tempered distributions. We have further, at times, mentioned modulation spaces, in particular Sobolev spaces, that provide a resolution of the space of rapidly decaying functions. In the the following, we will address a different functional setting, that is Gelfand-Shilov functions and ultradistributions of type $\mathscr{S}$. Since these ultradistributions share many features with the tempered distributions, it is a natural question if some of the preceding results may be generalized to them. For that, depending on the "degree of analyticity" one imposes, various techniques to (micro-)localize need to be replaced. Since the study of microlocal properties of Gelfand-Shilov type ultradistributions is a vast subject on its own, we will only discuss this in a brief and by no means complete fashion here.

Gelfand-Shilov spaces (of type $\mathscr{S}$ ), which are subspaces $\mathscr{S}\left(\mathbb{R}^{d}\right)$, were treated in great detail in [63], see also references to earlier works therein. Their dual spaces constitute the tempered ultradistributions, which generalize $\mathscr{S}^{\prime}\left(\mathbb{R}^{d}\right)$, consider also [90, 91, 92]. We introduce these spaces of functions and tempered ultradistributions as in [113, Chap. 6], making use of the characterization obtained in [24].

Definition 4.1. A function $f \in \mathscr{S}\left(\mathbb{R}^{d}\right)$ is an element of $\mathscr{S}_{\nu}^{\mu}\left(\mathbb{R}^{d}\right), \mu>0, \nu>0$, if there exists $\varepsilon>0$ such that

$$
|f(x)| \lesssim e^{-\varepsilon|x|^{1 / \nu}} \quad \text { and } \quad|\mathcal{F} f(\xi)| \lesssim e^{-\varepsilon|\xi|^{1 / \mu}}
$$


or equivalently if $\exists C^{\prime}, C>0$ such that $\forall \alpha, \beta \in \mathbb{N}_{0}^{d}$

$$
\left|x^{\alpha} \partial_{x}^{\beta} f(x)\right| \leq C^{\prime} C^{|\alpha|+|\beta|}(\alpha !)^{\nu}(\beta !)^{\mu} .
$$

These spaces are non-trvial ${ }^{63}$ if and only if $\mu+\nu \geq 1$. One also defines spaces for fixed $C>0$ in (4.1) and equip these with norms given by the best possible constants $C^{\prime}$. Then $\mathscr{S}_{\nu}^{\mu}\left(\mathbb{R}^{d}\right)$ is equipped with the topology of the inductive limit of these Banach spaces.

The corresponding topological dual, denoted by $\left(\mathscr{S}_{\nu}^{\mu}\right)^{\prime}\left(\mathbb{R}^{d}\right)$, is the space of tempered ultradistributions.

For an in-depth analysis of these spaces, we refer the reader to [63, 113]. They share many properties with $\mathscr{S}\left(\mathbb{R}^{d}\right)$ and $\mathscr{S}^{\prime}\left(\mathbb{R}^{d}\right)$, in particular $\mathcal{F}$ extends to a continuous isomorphism

$$
\mathcal{F}:\left\{\begin{array}{l}
\mathscr{S}_{\nu}^{\mu}\left(\mathbb{R}^{d}\right) \rightarrow \mathscr{S}_{\mu}^{\nu}\left(\mathbb{R}^{d}\right) \\
\left(\mathscr{S}_{\nu}^{\mu}\right)^{\prime}\left(\mathbb{R}^{d}\right) \rightarrow\left(\mathscr{S}_{\mu}^{\nu}\right)^{\prime}\left(\mathbb{R}^{d}\right) .
\end{array}\right.
$$

Furthermore, the spaces may be studied using methods of time-frequency analysis, and there are again resolutions in terms of modulation spaces, see e.g. 229, 141, 142, 143 .

We will now give a brief overview of how the concepts of microlocal analysis carry over to these classes of ultradistributions. We restrict our attention to the Fouriersymmetric case, and set $\mu=\nu=: \theta \geq 1 / 2$ and $\mathscr{S}_{\theta}\left(\mathbb{R}^{d}\right):=\mathscr{S}_{\theta}^{\theta}\left(\mathbb{R}^{d}\right)$.

By the Denjoy-Carleman theorem, see [81, Thm 1.3.8], $\mathscr{S}_{\theta}\left(\mathbb{R}^{d}\right)$ does not contain non-trivial compactly supported functions for $\theta \leq 1$, therefore we cannot find a class of cut-offs which are multipliers in this case. In fact, we need to consider three fundamental cases, which we discuss separately:

- $\theta>1$, where many of the previous techniques may still be employed, in particular we may employ (Gevrey) cut-offs,

- $\theta=1$, in which $\mathscr{S}_{\theta}$ coincides with the real analytic functions of exponential decay, and in which consequently methods from the theory of (Fourier) hyperfunctions may be employed,

- $\theta<1$, the quasi-analytic case, in which however several of these techniques break down and cannot be generalized.

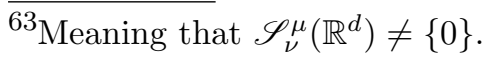


Remark 4.2. We note that it is also possible to work in more general spaces, such as the ultradistributions of Beurling type. These are essentially obtained by replacing the factorials in (4.1) by other suitable sequences $\gamma_{n}$, and yield further quasi-analytic cases when " $\gamma_{n} \approx n !$ !.

The case of $\theta>1$ :

In this case, many of the previous techniques may be generalized. For example, there are adapted generalizations of the SG-pseudo-differential calculus (see [20, 21, 113] and in particular classes of Gevrey cut-offs and adapted classes of (asymptotic) cut-offs ${ }^{64}$

Consequently, $\mathrm{WF}_{\mathrm{cl}}$ has an analogue in these spaces, the Gevrey-wave front set, which was already established in [78], see also [81, Sect. 8.4.] and [120].

The SG-wave front set was then generalized to these spaces in terms of Gevrey cut-offs and pseudo-differential operators, see [21], and many concepts may be generalized to these spaces, in particular SG-Fourier Integral operators, see [18, 19]. One thing to note is that statements in the smooth setting whose proofs are obtained by integral regularizations often need to be adapted, since the regularization needs to be "up to infinite order". One way to overcome such issues is by use of ultradifferential operators.

In various sources, such as [23, 99, 109], the Gevrey (or Denjoy-Carleman) wave front set for ultradistributions was characterized by means of an FBI-transform and it seems likely that this may be generalized to the SG-wave front set as well, as in Section 2.3.1

In [109], the formulation of $\mathrm{WF}_{\mathrm{G}}(u)$ in terms of the short time Fourier transform was extended to Gelfand-Shilov spaces.

The case of $\theta=1$ :

In this case, we have that each $f \in \mathscr{S}_{1}\left(\mathbb{R}^{d}\right)$ extends to an entire analytic function in some strip $\{x+i y|x \in \mathbb{R}| y \mid,<T\}$ for some $T>0$, which is exponentially decaying in $x$ (see e.g. [113, Prop. 6.1.8.]). Consequently, $\mathscr{S}_{1}^{\prime}\left(\mathbb{R}^{d}\right)$ coincides with the space of Fourier hyperfunctions, as introduced by Kawai [89] based on the works of Sato [124, 125]. This allows for the use of various techniques from sheaf theory and complex analysis.

\footnotetext{
${ }^{64}$ These are obtained by use of Gevrey functions (on both $\mathbb{R}^{d}$ and $\mathbb{S}^{d-1}$ ) in Construction 1.3
} 
We may obtain the notion of an analytic wave front set in several equivalent 65 ways, also called singular spectrum, see [81, Chap. 9], [147] and [89]. We will not go into much detail here considering techniques from hyperfunction theory, but mention that the analytic wave front set may be characterized by

(1) analyticity of (microlocal) boundary value representations in terms of analytic functions,

(2) boundary behaviour a fixed boundary value representation in terms of a convolution operator (see [81, Def. 9.3.2.]),

(3) decay properties of the FBI-transform (see also [15]).

(4) sequences of approximating distributions with increasing regularity, as well as pseudo-differential methods (see [147, Sect. 3.1.] and for tempered distributions [81, Def. 8.4.3.]).

The essential ingredient in all of these constructions is the concept of localizability of hyperfunctions. Again, these notions of analytic wave front set may then be used to establish operations on hyperfunctions, see e.g. [147, Sect. 3.4.].

We may thus attempt to generalize global wave front sets. Fourier hyperfunctions are already introduced in terms of the compactification $\mathbb{R}^{d} \sqcup\left(\mathbb{R}^{d} \backslash\{0\}\right)$ and the analytic wave front set generalizes to a subset of the compactification $\mathbb{B}^{d} \times \mathbb{S}^{d-1}$, see [89, 110]. This may be obtained in accordance with (1) in terms of regularity of microlocal boundary value representations, as in [88], and also by the approach (2), see [110]. It also seems very plausible that $\mathrm{WF}_{\mathrm{SG}}$ may be generalizable in terms of the FBI-transform and by approximating sequences of (asymptotic) cutoffs and pseudo-differential methods.

For $\mathrm{WF}_{\mathrm{G}}$, there exists again an analytic analogue in terms of the FBI transform, see [100, 101].

The case of $\theta<1$ :

In the case $\theta<1$, the situation changes drastically. There is no adequate notion of support for general ultradistributions in these spaces (as also metioned [82]) and consequently also no notion of singular support. There exist, however, various concepts to generalize the classical wave front set to capture local singularities of

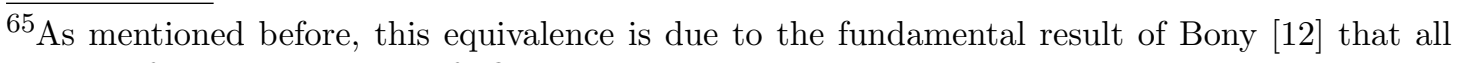
notions of singular spectrum fulfilling certain criteria all coincide.
} 
this type for distributions in $\mathscr{D}^{\prime}\left(\mathbb{R}^{d}\right)$, see [115, 116], using the localization techniques available for these spaces.

For general ultradistributions, there is, to the best of our knowledge, no such notion. Many techniques simply break down - for example the convolution of $(2)$ in the previous discussion can not be extended to $\theta<1$. There is also (to the best of our knowledge) no adapted partial differential calculus for these spaces.

The approach via the FBI-transform seems like a good candidate for generalization, since the transform extends to $u \in \mathscr{S}_{\theta}^{\prime}\left(\mathbb{R}^{d}\right)$ for $\theta<1$, by $\psi_{0} \in \mathscr{S}_{1 / 2}$. In the following example, we highlight the difficulties in obtaining a suitable generalization of $\mathrm{WF}_{\mathrm{cl}}$ for this approach. For that, we first state (see e.g. [109]) how the FBI-transform may be used to characterize Gevrey singularities of type $\theta \geq 1$ :

Proposition 4.3. Let $u \in \mathscr{S}^{\prime}\left(\mathbb{R}^{d}\right), \theta \geq 1$. Let $x_{0} \in \mathbb{R}^{d}, \xi_{0} \in \mathbb{R}^{d}$ with $\xi_{0} \neq 0$. Then $u$ is microlocally $\mathscr{S}_{\theta}$-regular at $\left(x_{0}, \xi_{0} \infty\right)$ if and only if there is an open neighbourhood $U \subset \mathbb{R}^{d} \times \mathbb{R}^{d}$ of $\left(x_{0}, \xi_{0}\right)$ for which $\exists \varepsilon, R>0$ such that for $\lambda>R$

$$
\left|\mathscr{F}_{\lambda, 1}(u)\right|_{U}(x, \xi) \mid \lesssim e^{-\varepsilon \lambda^{1 / \theta}}
$$

Example 4.4. We see that for $u=\delta_{0}$ we have

$$
\left|\mathscr{F}_{\lambda, 1}(u)(x, \xi)\right|=(2 \pi)^{d / 2} \lambda^{3 d / 4} \psi_{0}\left(\lambda^{1 / 2} x\right),
$$

which decays like $e^{-\lambda \varepsilon}$ outside of $x=0$, thus verifying that its analytic wave front set is $\{0\} \times\left(\mathbb{R}^{d} \backslash\{0\}\right)$.

However, if we simply extend the requirement 4.2 without modification to $\theta<1$, then $u$ is singular on all of $\mathbb{R}^{d} \times\left(\mathbb{R}^{d} \backslash\{0\}\right)$, not only at $x=0$. This reflects the lack of locality in these spaces: a singularity at any fixed point is "seen" at all other points.

Being unable to generalize the classical wave front set in a desirable way to these kinds of ultradistributions, there is of course no hope to generalize $\mathrm{WF}_{\mathrm{SG}}$.

For the G-wave front set, the previous problems do not arise.66 The definition in terms of the FBI-transform or STFT may be generalized in a straight forward manner, see [82] for the case $\theta=1 / 2$. In [22], we have complimented this definition by a consideration of the cases $1 / 2<\theta<1$. One way to define an adapted G-wave front set for these spaces is the following:

\footnotetext{
${ }^{66}$ This may be traced back to the different shape of characteristic sets therein, see Figure 11
} 
Definition 4.5. $u \in \mathscr{S}_{\theta}\left(\mathbb{R}^{d}\right)$ is G-microlocally $\mathscr{S}_{\theta}$-regular at $\left(x_{0}, \xi_{0}\right) \in \mathrm{W}_{\mathrm{G}}$ if and only if there exists a (conic) neighbourhood $\Gamma$ of $\left(x_{0}, \xi_{0}\right)$ such that $\exists \varepsilon>0$ for which

$$
\left|\mathscr{V}_{\psi_{0}} u(x, \xi)\right| \lesssim e^{-\varepsilon\left(|x|^{1 / \theta}+|\xi|^{1 / \theta}\right)} \quad(x, \xi) \in \Gamma .
$$

This notion of wave front set may be used to obtain analogous results to many of those contained in Section 2.5 in this functional setting. For that, one often replaces the STFT by an FBI transform with complex phase to prove invariance results and to make use of the analyticity. We refer the reader to [22, 82] for the details. In [22], we also discuss microlocality results for various operators, in particular localization (or Toeplitz-) operators, which is of particular interest in this functional setting, since there is no adapted pseudodifferential calculus, see also [29]. 


\subsection{General outlook.}

In this section, we will discuss further open ends and interesting starting points for a continuation of our analysis.

We have already mentioned that there are many different kinds of global wave front sets, and some of these notions were - to the best of our knowledge - not studied in great detail so far, for instance the metaplectic wave front set of [149]. There are also inhomogeneous calculi, where the $x$ and $\xi$ variables are weighted with different scaling exponents. Consequently, different topologies "at infinity" would arise in the compactifications. It would be interesting, in the future, to see which of our methods to characterize wave front sets and to study operations with it may be applicable also for such other notions, and which have "universal" character.

Considering the characterization of the SG-wave front set in Section 2.3, we note that the (standard) FBI transform is closely related to the so called wave packet transform, consider [37], which in its simplest form takes the form (for $f \in \mathscr{S}\left(\mathbb{R}^{d}\right)$ )

$$
(x, \xi) \mapsto c_{n}\langle\xi\rangle^{d / 4} \int f(y) e^{i \xi(x-y)} e^{-\frac{1}{2}\langle\xi\rangle(x-y)^{2}} d y .
$$

This transform has the property that it transforms the action of a classical pseudodifferential operator into multiplication by its symbol and terms of lower order. To unify global and local singularities, the following transform was suggested (in the framework of the homogeneous wave front set) in [100]

$$
(x, \xi) \mapsto \int f(y) e^{i \xi(x-y)} e^{-\frac{\langle\xi\rangle}{\langle x\rangle}(x-y)^{2}} d y .
$$

It seems very likely that using such a transform and the SG-calculus would yield a similar characterization of $\mathrm{WF}_{\mathrm{SG}}(u)$ as in Theorem 2.22 in terms of rapid decay of this wave packet transform. In [61, Chap. 3.2] it is mentioned that, by an observation of Weinstein [149], it is possible to characterize the wave front set in terms of decay of the Wigner transform. It is again very likely that this may be generalized, employing the techniques of Section 2.3.1, to the SG-wave front set. Using these transforms, it also seems likely that they might lead to a better understanding of the symbol construction in Section 3.3.1.

Another approach to study $\mathrm{WF}_{\mathrm{SG}}$ in the time-frequency plane can be found in the series of papers [40, 41, 42]. Therein, the integral transform used is the (unscaled) 
short-time Fourier transform. To achieve (micro-)localization, again a cut-off function is used and the resulting transformed expression is tested for decay in a cone. This framework yields both global as well as local wave front sets with respect to various function spaces $E$ with $\mathscr{S}\left(\mathbb{R}^{d}\right) \subset E \subset \mathscr{S}^{\prime}\left(\mathbb{R}^{d}\right)$, in particular a resolution of $\mathrm{WF}_{\mathrm{SG}}$ in terms of modulation spaces. The approach has been further used to study the propagation of singularities under the action of SG-Fourier Integral operators in [43]. It seems likely that our methods using the FBI-transform may be applicable as well with respect to these functional settings.

In Section 2.4.1 we have already mentioned that the microlocal existence criteria we have established are far from being necessary conditions. One fairly simple way of generalizing them further would be taking "microlocally compatible spaces" into account, that is considering at each point different wave front sets, e.g. with respect to Sobolev spaces. In particular, if one views the product as a restriction of $u \otimes v$ to the diagonal $\left\{(x, x) \mid x \in \mathbb{R}^{d}\right\} \subset \mathbb{R}^{d} \times \mathbb{R}^{d}$ in terms of the pull-back by $\delta: x \mapsto(x, x)$, then the tensor product is always well defined, the restriction however is not. The well-known Sobolev trace theorem provides us with an existence criterion for such restrictions in terms of Sobolev regularity. Imposing Sobolev regularity microlocally at a submanifold naturally leads into the field of study of 2-microlocal analysis, see [13, 93]. We hope to be able to strengthen our results using such techniques in the future.

For $\mathrm{WF}_{\mathrm{G}}$, the situation is similar. In the Gelfand-Shilov setting, there is so far only the characterization in terms of the FBI/STFT-transforms. It remains to be checked if the wave front set is also characterizable by adapted pseudodifferential calculi, for $\theta>1$, or by localization operators and Gabor frames. It also remains to be seen if one is able to prove a micro-ellipticity-type result for localization operators. We will address the latter issue in future studies.

For both wave front sets, there already exist plenty of applications to the theory of partial differential equations. It is our hope that the techniques discussed in this thesis will be applicable to obtain further results on propagation of singularities and (unique) solvability.

In Section 3.2 we have defined a tempered version of oscillators integrals and Lagrangian submanifolds on Euclidean spaces. This rich field of study opens up many unresolved questions. First of all, it is important to define a class of invariantly defined Lagrangian distributions and in particular to obtain a symbol map for 
them. The next step would be to construct a corresponding operator theory, with particular emphasis on applications to partial differential as a consequent further step. In particular, one may study Fourier integral operators of the form

$$
\mathscr{S}\left(\mathbb{R}^{d}\right) \ni u \mapsto \int e^{i \varphi(x, \theta)} a(x, \theta) \hat{u}(\theta) d \theta
$$

and their transposes.

Using the results of Section 2.4.1, one may extend these operators to tempered distributions under suitable assumptions on their wave front set, in terms of $\Lambda_{\varphi}$. Operators of this kind "of type I" with very regular phases, i.e.

$$
\left\langle\nabla_{x} \varphi\right\rangle \asymp\langle\theta\rangle \quad\left\langle\nabla_{\theta} \varphi\right\rangle \asymp\langle x\rangle
$$

have been introduced in [38], see also [1, 39, 45, 46, 47] and the propagation of the SG-wave front set under the action of such operators has been studied in [43, 44]. Our assumptions on SG-phases are far less restrictive than (4.4), and in particular the solution operator to the Cauchy problem of the free Klein-Gordon-equation on $\mathbb{R}^{d+1}$, where the time is not treated as a parameter but as a space-time variable, may be brought into the form 4.3). First steps in this direction of research have been made in [49]. With a fully developed calculus at hand, we will be able to study global (in time and space) solutions to such equations and various problems from scattering theory very efficiently. We will address such questions in future publications.

Generally speaking, we have made, throughout this thesis, thorough use of properties and tools unique to Euclidean spaces, such as the Fourier transform and time-frequency shifts. Nevertheless, we are confident that several of our findings may be generalized to asymptotically flat, or scattering manifolds, such as in e.g. [107]. In particular there exists a notion of FBI-transform on compact manifolds, see [155]. Connections of our findings considering SG-Lagrangians with results that have been achieved from the viewpoint of the theory of Legendrian distributions, see [71, 70, 107], remain to be established.

In Section 3.3 we have discussed the scaling behaviour of a distributions. There are several directions in which one could pursue the investigation of the SG-symbol in 3.3.1. So far, all that has been shown is how the definitions of [148] relate to the generalized FBI-transform, so there are quite a few starting points for further 
investigations, in particular one could check if the SG-symbol for tempered oscillatory integrals of Section 3.2, in analogy to the results in [148], will coincide with a suitable global notion of symbol for the SG-Lagrangian distributions. Another aspect of study would be an analogous G-symbol and an investigation of the properties and applicability of such a notion, especially for Shubin-type Fourier operators, see e.g. [31].

Concerning the extensions of distributions in terms of the scaling degree of Section 3.3.2, we have already mentioned that it is possible to combine these methods with microlocal considerations, in particular to bound the wave front set of the resulting distributions, and to use this theory to renormalize products of (tempered) distributions defined outside a subspace. This remains to be carried out in detail for tempered distributions. How this renormalized product relates to the notion of product in the framework of Colombeau's algebras of generalized functions, [25, 26, 27], also remains to be clarified.

It has already been noted in Remark 3.55 that a particular case in which it is possible to control the wave front set of the extended distribution is under certain conormality conditions. It is known that the deviation of a distribution from being conormal may be measured in terms in the framework of 2-microlocal analysis, see [13, 93]. It is a remarkable feature that in the case of a single point $x_{0}$, the spaces of weakly homogeneous distributions arising in the continuation procedure, see [108], are deeply connected with the two-microlocal spaces $C_{x_{0}}^{s, s^{\prime}}$, which are a generalization of Hölder-Zigmund-spaces cf. [146, Rem. 3.40], in terms of which two-microlocal regularity is measured. It is therefore reasonable to assume that techniques from 2-microlocal analysis may be applied to the extension procedure, and in particular the renormalization of the product, for which we have already discussed how 2-microlocal analysis may yield a more general existence criterion. This connection will be subject to further investigation.

It is further interesting to study if this extension procedure is applicable to the problem of existence of tempered solutions for non-linear partial differential equations involving products of distributions, such as Burger's equation or differential equations with distributional coefficients.

In Section 3.5, we have discussed some applications of our techniques to the theory of quantum fields. Therein, we have only treated some examples and pointed out several directions in which it is possible to generalize the analysis, such as the 
study of the adiabatic limit. We have demonstrated that our methods provide an efficient framework to define various distributions from QFT by reducing the analysis to simple algebraic criteria on the involved wave front sets. In order to apply these concepts in a more general framework, it would again be of great interest to first generalize our methods to manifolds, in particular globally hyperbolic manifolds with prescribed asymptotic behaviour, in order to be able to study models from quantum field theory on curved spacetimes. With that, it would be very interesting to complement results of [16, 74, 75, 112, 152] by taking into account asymptotic features of distributions.

Additionally, it would be interesting, especially for applications to partial differential equations to also study different functional settings, such as Modulation spaces, Gelfand-Shilov spaces, the analytic framework, etc. In each of these frameworks, there are interesting new phaenomena to consider. We mention as example that the sheet of (Fourier) hyperfunctions is flabby, meaning that any such element defined on $\mathbb{R}^{d} \backslash X$ admits some extension, which is not the case for tempered distributions, for which we assumed finiteness of the scaling degree, see Section 3.3.2. In the Gelfand-Shilov framework for any $\theta \geq \frac{1}{2}$, one is able to consider the Weyl operators with (ultra-)distributional symbol of Section 3.4. We have seen in Proposition 3.63, that under reasonable assumptions on the G-wave front set of the kernels, we obtain a class of operators closed under composition. This is of particular interest in the quasi-analytic case, since there is no adapted symbol class for a pseudodifferential calculus available in the quasianalytic framework. 


\section{Appendix A. Collection of auxiliary Results}

\section{A.1. Notation, spaces of test functions and distributions.}

In the following, some basic notation is collected that is used throughout this thesis. For the reader's convenience, recurring notation is also collected in Appendix B,

- We use the notation $i=\sqrt{-1}, \mathbb{N}_{0}=\{0,1,2, \ldots\}, \mathbb{R}_{+}=\{x \in \mathbb{R} \mid x>0\}$, $\mathbb{B}^{d}=\{y \in \mathbb{R}|| y \mid \leq 1\}, \mathbb{S}^{d-1}=\partial \mathbb{B}^{d}=\{y \in \mathbb{R}|| y \mid=1\}$.

- We use standard multi-index notation and $\delta_{j, k}= \begin{cases}1 & j=k \\ 0 & j \neq k\end{cases}$

- We set $D_{x}^{\alpha}=i^{|\alpha|} \partial_{x}^{\alpha}$ for multi-indices $\alpha \in \mathbb{N}_{0}^{d}$ and $\nabla$ for the gradient on $\mathbb{R}^{d}$, i.e. in coordinates $\nabla_{x}=\left(\partial_{x_{1}}, \ldots, \partial_{x_{d}}\right)^{t}$.

- Let $X$ be a subset on $\mathbb{R}^{d}$ that admits a smooth structure, such as a manifold (with boundary). Then $X^{o}$ denotes its interior and $\partial X$ its boundary.

- The symbol $\mathscr{E}(X)=\mathscr{C}^{\infty}(X)$ stands for the space of all smooth functions on $X$, and $\mathscr{D}(X)=\mathscr{C}_{c}^{\infty}(X)$ for those that are compactly supported in the interior of $X$. Both spaces are equipped with the usual topologies that turn them into Fréchet spaces.

- For two maps $f, g: X \rightarrow[0, \infty)$ we say $f \lesssim g$ if there exists $C>0$ such that for all $x \in X f(x) \leq C g(x)$, and $f \asymp g$ means that $f \lesssim g$ and $g \lesssim f$. If $f$ and $g$ depend on indices, then so may $C$.

- The Japanese bracket is given by the map $\langle\cdot\rangle: \mathbb{R}^{d} \rightarrow \mathbb{R}_{+}, x \mapsto\langle x\rangle:=$ $\sqrt{1+|x|^{2}}$, where $|x|$ denotes the Euclidean norm on (subsets of) $\mathbb{R}^{d}$. For this map we have Peetre's inequality, i.e. $\forall x, y \in \mathbb{R}^{d}, k \in \mathbb{Z}$

$$
\langle x+y\rangle^{k} \lesssim\langle x\rangle^{k}\langle y\rangle^{|k|}
$$

- The symbol $(\cdot, \cdot)$ denotes the complex $L^{2}$-scalar product between functions, vectors etc. and restrictions and extensions of it to other spaces. The real pairing is denoted by $\langle\cdot, \cdot\rangle$.

- The letter $\omega$ denotes the canonical two-form on the cotangent bundle $T^{*} X$ of a manifold $X$.

For $X=\mathbb{R}^{d}$ we define $\omega: \mathbb{R}^{2 d} \times \mathbb{R}^{2 d} \rightarrow \mathbb{R}: \omega((x, \xi) ;(y, \eta))=\langle\xi, y\rangle-\langle\eta, x\rangle$.

- We indicate by $\mathbb{1}$ both the constant unit function as well as unit matrices, and by $\mathbb{1}_{X}$, for $X \subset \mathbb{R}^{d}$, the indicator function, i.e. $\mathbb{1}_{X}(x)= \begin{cases}1 & x \in X \\ 0 & x \notin X\end{cases}$ 


\section{A.2. Schwartz functions and tempered distributions.}

In the following, we have collected some established facts about Schwartz functions and tempered distributions. Since we will not include proofs, we refer to [61, 62, 68, 181, 122, 117, 132, 135] as general references for the upcoming statements.

Definition A.1 (Schwartz functions and tempered distributions). A smooth function $f \in \mathscr{C}^{\infty}\left(\mathbb{R}^{d}\right)$ is called to be of rapid decay, or a Schwartz (test) function, if it satisfies $\sup _{x \in \mathbb{R}^{d}}\left|x^{\alpha} \partial_{x}^{\beta} f\right|<\infty$ for all multi-indices $\alpha, \beta \in \mathbb{N}_{0}^{d}$.

The Fréchet space of all Schwartz functions is denoted by $\mathscr{S}\left(\mathbb{R}^{d}\right)$, and a family of semi-norms $\left\{\rho_{N}^{\mathscr{S}}\right\}_{N \in \mathbb{N}_{0}}$ is given by

$$
\rho_{N}^{\mathscr{S}}(f)=\sup _{x \in \mathbb{R}^{d}} \sum_{|\alpha|+|\beta| \leq N}\langle x\rangle^{|\alpha|}\left|\partial_{x}^{\beta} f\right| .
$$

The space of tempered distributions $\mathscr{S}^{\prime}\left(\mathbb{R}^{d}\right)$ is defined as the topological dual of $\mathscr{S}\left(\mathbb{R}^{d}\right)$, i.e. all $\mathbb{C}$-linear maps $u: \mathscr{S}\left(\mathbb{R}^{d}\right) \rightarrow \mathbb{C}$ such that $|u(f)| \lesssim \rho_{N}^{\mathscr{S}}(f)$ for some $N \in \mathbb{N}_{0}$. The real pairing between distributions and test functions is denoted by $u(f)=:\langle u, f\rangle$.

In the following, we list some well-established facts about Schwartz functions and tempered distributions, see in particular [117, Chap. V]:

Proposition A.2 (Topological and embedding properties of tempered distributions).

(1) A family of seminorms on $\mathscr{S}\left(\mathbb{R}^{d}\right),\left\{\rho_{N, M}^{\mathscr{S}}\right\}_{(N, M) \in \mathbb{N}_{0}^{2}}$, equivalent to the one defined by (A.2) is given by

$$
\rho_{N, M}^{\mathscr{S}}(f)=\sup _{x \in \mathbb{R}^{d}} \sum_{|\alpha| \leq N} \sum_{|\beta| \leq M}\langle x\rangle^{|\alpha|}\left|\partial_{x}^{\beta} f\right| .
$$

(2) $\mathscr{S}^{\prime}\left(\mathbb{R}^{d}\right)$ may be equipped with the weak-*-topology, which induces the following notion of convergence of distributions: a sequence of tempered distributions $\left(u_{n}\right)_{n \in \mathbb{N}_{0}}$ converges to $u \in \mathscr{S}^{\prime}\left(\mathbb{R}^{d}\right)$ if we have $\left\langle u_{n}, f\right\rangle \rightarrow\langle u, f\rangle$ for all $f \in \mathscr{S}\left(\mathbb{R}^{d}\right)$.

(3) (Banach-Steinhaus-principle) A subset $\left\{u_{\lambda}\right\} \subset \mathscr{S}^{\prime}\left(\mathbb{R}^{d}\right)$ is bounded if $\forall f \in$ $\mathscr{S}\left(\mathbb{R}^{d}\right)$ there exists a constant $C(f)$ such that $\sup _{\lambda}\left\{\left\langle u_{\lambda}, f\right\rangle\right\} \leq C(f)$,

(4) We have the dense inclusions $\mathscr{E}\left(\mathbb{R}^{d}\right) \subset \mathscr{S}\left(\mathbb{R}^{d}\right) \subset \mathscr{D}\left(\mathbb{R}^{d}\right)$ and consequently $\mathscr{D}^{\prime}\left(\mathbb{R}^{d}\right) \subset \mathscr{S}^{\prime}\left(\mathbb{R}^{d}\right) \subset \mathscr{E}^{\prime}\left(\mathbb{R}^{d}\right)$. 
(5) We have the embedding of continuous, polynomially bounded functions

$$
\mathscr{C}_{\mathrm{pol}}\left(\mathbb{R}^{d}\right) \hookrightarrow \mathscr{S}^{\prime}\left(\mathbb{R}^{d}\right) \text { via } g \mapsto\left(f \rightarrow \int_{\mathbb{R}^{d}} g(x) f(x) d x\right) .
$$

(6) $\mathscr{S}\left(\mathbb{R}^{d}\right)$ is dense in $L^{2}\left(\mathbb{R}^{d}\right)$. In fact we have a family of $\mathscr{S}\left(\mathbb{R}^{d}\right)$-functions, the Hermite functions $\left\{\psi_{\alpha}\right\}_{\alpha \in \mathbb{N}_{0}^{d}}$, defined via

- $\psi_{0}(x):=\pi^{-d / 4} e^{-\frac{x^{2}}{2}} \in \mathscr{S}\left(\mathbb{R}^{d}\right)$ being the normalized Gaussian,

- $\psi_{\alpha}:=(\alpha !)^{\frac{1}{2}}\left(\frac{x-\partial_{x}}{\sqrt{2}}\right)^{\alpha} \psi_{0}$,

which form an orthonormal basis of $L^{2}\left(\mathbb{R}^{d}\right)$. In particular we have that $f=\sum_{\alpha \in \mathbb{N}_{0}^{d}} a_{\alpha} \psi_{\alpha}$ is in $\mathscr{S}\left(\mathbb{R}^{d}\right)$ if and only if the Hermite coefficient ${ }^{67} a_{\alpha}$ satisfy $\sup _{\alpha \in \mathbb{N}_{0}^{d}}\left|a_{\alpha}\right||\alpha|^{k} \leq \infty$ for all $k \in \mathbb{N}_{0}$.

(7) $\mathscr{S}\left(\mathbb{R}^{d}\right)$ equipped with the family of semi-norms $\left\{\rho_{N}\right\}_{N \in \mathbb{N}_{0}}$ is a nuclear space and we have $\mathscr{S}\left(\mathbb{R}^{d}\right) \widehat{\otimes} \mathscr{S}\left(\mathbb{R}^{d^{\prime}}\right) \cong \mathscr{S}\left(\mathbb{R}^{d} \times \mathbb{R}^{d^{\prime}}\right)$.

(8) $\mathscr{S}\left(\mathbb{R}^{d}\right)$, under the embedding A.4, is dense in in $\mathscr{S}^{\prime}\left(\mathbb{R}^{d}\right)$. We call an element of $\mathscr{S}^{\prime}\left(\mathbb{R}^{d}\right)$ that is given by some $f \in \mathscr{S}\left(\mathbb{R}^{d}\right)$ (globally) $\mathscr{S}$-regular.

These topological properties allow for a definition of analogues of operations on functions in the space of distributions. This is normally done either

- by duality, meaning for a map $A: \mathscr{S}\left(\mathbb{R}^{d}\right) \rightarrow \mathscr{S}\left(\mathbb{R}^{d}\right)$ with continuous $L^{2}$-transpose one sets $\langle A u, f\rangle=\left\langle u,{ }^{t} A f\right\rangle$ or

- by continuous extension ${ }^{68}$ meaning one sets for an approximative sequence $g_{n} \rightarrow u$ the distribution $\langle A u, f\rangle=\lim _{n \rightarrow \infty}\left\langle A g_{n}, f\right\rangle$.

(1) We have the Fourier transform, which yields an isomorphism on $\mathscr{S}\left(\mathbb{R}^{d}\right)$ and $\mathscr{S}^{\prime}\left(\mathbb{R}^{d}\right)$ respectively, with the notation and normalization

$$
\mathcal{F} f(\xi)=\widehat{f}(\xi)=\int_{\mathbb{R}^{d}} e^{-i x \xi} f(x) d x \quad f \in \mathscr{S}\left(\mathbb{R}^{d}\right)
$$

and $\langle\mathcal{F} u, f\rangle:=\langle u, \mathcal{F} f\rangle$.

(2) There exists a natural product $\mathscr{S}\left(\mathbb{R}^{d}\right) \times \mathscr{S}^{\prime}\left(\mathbb{R}^{d}\right) \rightarrow \mathscr{S}^{\prime}\left(\mathbb{R}^{d}\right)$ given by $\langle(f \cdot u), g\rangle=\langle u, f \cdot g\rangle$ which is sequentially continuous, i.e. if $f_{n} \stackrel{\mathscr{S}}{\rightarrow} f$ and $u_{n} \stackrel{\mathscr{S}^{\prime}}{\rightarrow} u$, then we have $f_{n} u_{n} \rightarrow f u$. We further have a natural notion of

\footnotetext{
$\overline{{ }^{67} \text { We recall that, cf. e.g. }[73},\left|\psi_{\alpha}(x)\right|$ is bounded independently of $x$ and $|\alpha|$ by a (dimensionaldependent) constant, thus the sum in the expansion converges absolutely for each $x \in \mathbb{R}^{d}$.

${ }^{68}$ Here, "continuous" means sequential continuity, that is referring to the notion of convergence of sequences of distributions recalled in Proposition A.2.
} 
convolution $\mathscr{S}\left(\mathbb{R}^{d}\right) \times \mathscr{S}^{\prime}\left(\mathbb{R}^{d}\right) \rightarrow \mathscr{S}^{\prime}\left(\mathbb{R}^{d}\right) \cap \mathscr{C}^{\infty}\left(\mathbb{R}^{d}\right)$ that satisfies

$$
\mathcal{F}(u * f)=(\mathcal{F} u) \cdot(\mathcal{F} f)
$$

(3) Differentiation yields a continuous map on $\mathscr{S}^{\prime}\left(\mathbb{R}^{d}\right)$ via

$$
\left\langle\partial^{\alpha} u, f\right\rangle=(-1)^{|\alpha|}\left\langle u, \partial^{\alpha} f\right\rangle .
$$

We further have the following regularity statements that provide a fairly explicit characterization of tempered distributions:

Proposition A.3. Let $u \in \mathscr{S}^{\prime}\left(\mathbb{R}^{d}\right)$.

- Then there exists a finite collection of $u_{\alpha \beta} \in L^{2}\left(\mathbb{R}^{d}\right)$ such that

$$
u=\sum_{\alpha, \beta} x^{\alpha} D^{\beta} u_{\alpha \beta} .
$$

- Similarly, u may be written, for some $\alpha \in \mathbb{N}_{0}^{d}$, as the $\alpha$-th (distributional) derivative of a continuous, polynomially bounded function $g \in \mathscr{C}_{\mathrm{pol}}\left(\mathbb{R}^{d}\right)$,

$$
u=\partial^{\alpha} g .
$$

The following famous Schwartz kernel theorem marks one of the main applications of distribution theory.

Theorem A.4 (Schwartz kernel theorem). There is a 1:1-correspondence between linear operators $A: \mathscr{S}\left(\mathbb{R}^{d}\right) \rightarrow \mathscr{S}^{\prime}\left(\mathbb{R}^{d^{\prime}}\right)$ and their kernels $\mathcal{K}_{A} \in \mathscr{S}^{\prime}\left(\mathbb{R}^{d} \times \mathbb{R}^{d^{\prime}}\right)$ given by

$$
\left\langle\mathcal{K}_{A}, f \otimes g\right\rangle=\langle A f, g\rangle .
$$

A.2.1. Basics of time-frequency analysis on tempered distributions.

In addition to the previously introduced operations, we have the following:

Definition A.5 (Isometries of the time-frequency plane). Let $f \in \mathscr{S}\left(\mathbb{R}^{d}\right)$. Then we can define the following operations:

- Modulation by $\xi_{0} \in \mathbb{R}^{d}:\left(\mathcal{M}_{\xi_{0}} f\right)(x)=e^{i \xi_{0} \cdot x} f(x)$,

- Translation by $x_{0} \in \mathbb{R}^{d}:\left(\mathcal{T}_{x_{0}} f\right)(x)=f\left(x-x_{0}\right)$,

- Dilation by $A \in \mathrm{GL}(d, \mathbb{R})$ or $\lambda>0: \mathcal{D}_{A} f(x)=\sqrt{|\operatorname{det}(A)|} f(A x)$ and $\left(\mathcal{D}_{\lambda} f\right)(x)=\left(\mathcal{D}_{\lambda \mathbb{1}} f\right)(x)=\lambda^{d / 2} f(\lambda x)$. 
Lemma A.6. All of the above maps are $L^{2}$-isometries with inverses given by their adjoints

$$
\mathcal{M}_{\xi_{0}}^{*}=\mathcal{M}_{-\xi_{0}} \quad \mathcal{T}_{x_{0}}^{*}=\mathcal{T}_{-x_{0}} \quad \mathcal{D}_{A}^{*}=\mathcal{D}_{A^{-1}}
$$

and can be defined on $\mathscr{S}^{\prime}\left(\mathbb{R}^{d}\right)$ by duality, i.e. $\langle U u, f\rangle=\left\langle u,{ }^{t} U f\right\rangle$. They admit the following behaviour under Fourier transformation:

$$
\mathcal{F} \circ \mathcal{D}_{A}=\mathcal{D}_{\left({ }^{t} A\right)^{-1}} \circ \mathcal{F} \quad \mathcal{F} \circ \mathcal{M}_{\xi}=\mathcal{T}_{\xi} \circ \mathcal{F} \quad \mathcal{F} \circ \mathcal{T}_{x}=\mathcal{M}_{-x} \circ \mathcal{F} .
$$

The time-frequency plane $T^{*} \mathbb{R}^{d}$ is the model space in which information about location and direction of singularities of a distribution are encoded. The so-called short-time Fourier transform (STFT), a basic tool of time-frequency analysis, see e.g. [61, 68, 69], can be used to obtain a representation of a tempered distribution over that space.

Definition A.7 (The short-time Fourier transform). Let $g \in \mathscr{S}\left(\mathbb{R}^{d}\right) \backslash\{0\}$, called the window function. Then the short-time Fourier transform $(\operatorname{STFT}) \mathscr{V}_{g}(u)$, for $u \in \mathscr{S}^{\prime}\left(\mathbb{R}^{d}\right)$, is defined as

$$
\mathscr{V}_{g}(u)(z)=\underbrace{\frac{1}{(2 \pi)^{d / 2}\|g\|_{2}}}_{=: C_{g}}\left(u, \mathcal{M}_{\xi} \mathcal{T}_{x} g\right) \quad \text { with } z=(x, \xi) .
$$

In particular we have for $u=f \in \mathscr{S}\left(\mathbb{R}^{d}\right)$

$$
\mathscr{V}_{g}(f)(x, \xi)=C_{g} \int_{\mathbb{R}^{d}} f(y) \overline{g(y-x)} e^{-i \xi \cdot y} d y .
$$

The standard (Gaussian) window is given by the normalized Gaussian $\psi_{0}(x)$.

Figure 18, fashioned after [68, Figure 3.5], provides a schematic illustration of the short-time Fourier transform.

Remark A.8. The STFT with standard window $\psi_{0}$ is closely related to several different transforms used in microlocal analysis, among them the Bargmann or Fourier-Bros-Iagolnitzer transform (short: FBI-transform). One such transform (with quadratic phase and index $\lambda$ ) takes the following form for $f \in \mathscr{S}\left(\mathbb{R}^{d}\right)$

$$
\mathscr{F}_{\lambda} f(x, \xi)=\underbrace{\left(\frac{\lambda}{2 \pi^{3 / 2}}\right)^{d / 2}}_{=: C_{\lambda}} \int_{\mathbb{R}^{d}} f(y) \psi_{0}\left(\lambda^{1 / 2}(x-y)\right) e^{-i \lambda \xi y} d y
$$




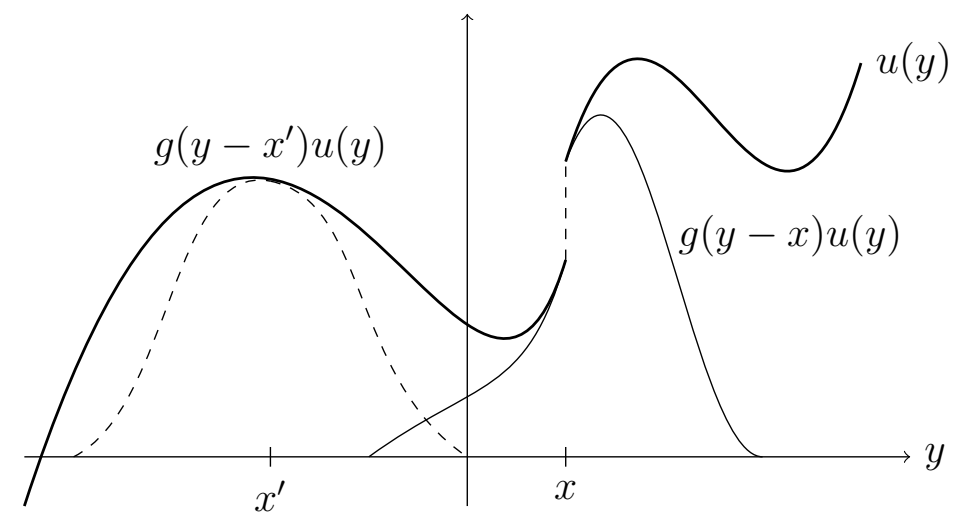

FiguRE 18. The short-time Fourier transform: localization by a window function (schematic)

and may thus be defined also for $u \in \mathscr{S}^{\prime}\left(\mathbb{R}^{d}\right)$ by (using A.10)

$$
\mathscr{F}_{\lambda} u(x, \xi)=\lambda^{d / 4} \mathscr{V}_{\mathcal{D}_{\lambda^{1 / 2}} \psi_{0}}(u)(x, \lambda \xi)=\mathcal{D}_{\lambda^{1 / 2}} \mathscr{V}_{\psi_{0}}\left(\mathcal{D}_{\lambda^{-1 / 2}} u\right)
$$

This transform (in a generalized form) will be the main tool in Section 2.3.1.

In the following Lemma, well-known properties of the STFT are listed, proofs for which can be found in [69] and in [68, Chap. 11.2].

Lemma A.9 (Properties of the STFT). For any window $g \in \mathscr{S}\left(\mathbb{R}^{d}\right)$ we have that the STFT is a continuous map $\mathscr{S}\left(\mathbb{R}^{d}\right) \rightarrow \mathscr{S}\left(\mathbb{R}^{2 d}\right)$ and $\mathscr{S}^{\prime}\left(\mathbb{R}^{d}\right) \rightarrow \mathscr{S}^{\prime}\left(\mathbb{R}^{2 d}\right)$. In fact $\mathscr{V}_{g} u$ for $u \in \mathscr{S}\left(\mathbb{R}^{d}\right)$ will be continuous and polynomially bounded.

We have the following orthogonality relation for $g_{i} \in \mathscr{S}\left(\mathbb{R}^{d}\right) \backslash\{0\}, f \in \mathscr{S}\left(\mathbb{R}^{d}\right)$, $u \in \mathscr{S}^{\prime}\left(\mathbb{R}^{d}\right)$ :

$$
\left(\mathscr{V}_{g_{1}}(u), \mathscr{V}_{g_{2}}(f)\right)=\frac{\left(g_{2}, g_{1}\right)}{\left\|g_{1}\right\|_{2}\left\|g_{2}\right\|_{2}}(u, f)
$$

In particular the STFT is an isometry $\left\|\mathscr{V}_{g} f\right\|_{L^{2}\left(\mathbb{R}^{2 d}\right)}=\|f\|_{L^{2}\left(\mathbb{R}^{d}\right)}$. Furthermore, if $\left(g_{1}, g_{2}\right) \neq 0$, the inversion formula, or Moyal identity

$$
u=C_{g_{2}} \frac{\left\|g_{1}\right\|_{1}\left\|g_{2}\right\|_{2}}{\left(g_{2}, g_{1}\right)}\left\langle\mathscr{V}_{g_{1}} u, \mathcal{M}_{\xi} \mathcal{T}_{x} g_{2}(\cdot)\right\rangle
$$

holds, which may also be written for $g_{1}=g_{2}=g$ as $\left(\mathscr{V}_{g}^{*}\right) \mathscr{V}_{g} u=u$ with the $L^{2}$ adjoint $\mathscr{V}_{g}^{*}$ of $\mathscr{V}_{g}$, which may be written as

$$
F \mapsto C_{g_{2}}\left\langle F, \mathcal{M}_{\xi} \mathcal{T}_{x} g_{2}(\cdot)\right\rangle,
$$


where the pairing is carried out with respect to the variables $(x, \xi) \in \mathbb{R}^{2 d}$.

The STFT admits the following transformation behaviour under time-frequency shifts and dilations:

$$
\begin{aligned}
\mathscr{V}_{g}\left(\mathcal{M}_{\xi_{0}} \mathcal{T}_{x_{0}} u\right)(x, \xi) & =e^{-i x_{0} \xi \mathscr{V}_{g}(u)\left(x-x_{0}, \xi-\xi_{0}\right)} \\
\mathscr{V}_{g}\left(\mathcal{D}_{\lambda} u\right)(x, \xi) & =\mathscr{V}_{\mathcal{D}_{\lambda-1} g}(u)\left(\lambda x, \lambda^{-1} \xi\right)
\end{aligned}
$$

The following estimate serves to estimate changes to another non-zero window $h \in \mathscr{S}\left(\mathbb{R}^{d}\right):$

$$
\left|\mathscr{V}_{g} u\right| \lesssim\|h\|_{2}^{-1}\left(\left|\mathscr{V}_{h} g\right| *\left|\mathscr{V}_{h} u\right|\right)
$$

The following proposition, which can be found in [68, Prop. 11.2.4] and [69], underlines how Schwartz functions may be constructed from a superposition of time-frequency shifts with a rapidly decaying amplitude, that is in terms of the adjoint $\mathscr{V}_{g}^{*}$. We have already seen a special case of this in the inversion formula.

Proposition A.10. Let $g \in \mathscr{S}\left(\mathbb{R}^{d}\right)$ non-zero. Assume $F \in L_{\text {loc }}^{\infty}\left(\mathbb{R}^{2 d}\right)$ satisfies the rapid decay condition

$$
|F(x, \xi)| \lesssim\langle(x, \xi)\rangle^{-N} \quad \forall N \in \mathbb{N}_{0} .
$$

Then $f$ defined via

$$
y \mapsto f(y):=C_{g}\left\langle F,\left(\mathcal{M}_{\xi} \mathcal{T}_{x} g\right)(y)\right\rangle,
$$

where the scalar product is carried out with respect to the variables $(x, \xi)$, is an element of $\mathscr{S}\left(\mathbb{R}^{d}\right)$.

Conversely, consider [68, Cor. 11.2.6], if we have for some $N \in \mathbb{N}_{0}$ that $F \in$ $L_{\text {loc }}^{\infty}\left(\mathbb{R}^{2 d}\right)$ satisfies the (polynomial) bound $|F(x, \xi)| \lesssim\langle(x, \xi)\rangle^{N}$ for some $N \in \mathbb{N}_{0}$, then we can set

$$
(u, f)=\left(F, \mathscr{V}_{g} f\right) .
$$

The latter expression is well-defined, since $F \in \mathscr{S}^{\prime}\left(\mathbb{R}^{2 d}\right)$ and $\mathscr{V}_{g} f \in \mathscr{S}\left(\mathbb{R}^{2 d}\right)$ by Lemma A.9 and can be shown to yield $u \in \mathscr{S}^{\prime}\left(\mathbb{R}^{d}\right)$. 


\section{A.3. Elements of pseudodifferential calculus.}

A.3.1. Pseudodifferential operators and parametrices. In this appendix, further facts on SG- and G-symbol classes as well as elements of the associated pseudodifferential calculi associated will be recalled. Since many authors have previously addressed this subject, we include it mainly to recall the results and to fix notation. The main reference for this section is [113, additional proofs and historical remarks can be found in e.g. [83, 94, 131, 135] and many others.

Since most concepts may be formulated in a similar fashion for both SG- as well as G-classes, we use the following abbreviation, to avoid repetitions.

Notation. Denote by SX a place-holder for either G and SG: all symbols SX in a statement my be replaced either by $\mathrm{G}$ or $\mathrm{SG}$ and the statement remains valid. The symbol $\mathrm{SX}^{m}$ specifies the order, i.e. $\mathrm{SX}^{m}$ can be replaced by either $\mathrm{G}^{m}$ or $\mathrm{SG}^{m_{e}, m_{\psi}}$. Furthermore, $\mathrm{SX}^{m-r}, r \in \mathbb{R}$, means either $\mathrm{G}^{m-r}$ or $\mathrm{SG}^{m_{e}-r, m_{\psi}-r}$. To shorten notation, we sometimes omit the base space from the notation of such a space when there can be no confusion, writing e.g. SX instead of $\operatorname{SX}\left(\mathbb{R}^{d} \times \mathbb{R}^{d}\right)$.

The first result allows for an approximation of symbols by such of lower order or test functions (cf. [113, Prop. 1.1.5]).

Lemma A.11. For any $\epsilon>0$ we have that $\mathscr{S}\left(\mathbb{R}^{d} \times \mathbb{R}^{d}\right)$ is dense in $\mathrm{SX}^{m}\left(\mathbb{R}^{d} \times \mathbb{R}^{d}\right)$ in the topology of $\mathrm{SX}^{m+\epsilon}\left(\mathbb{R}^{d} \times \mathbb{R}^{d}\right)$.

Consider first $a \in \mathscr{S}^{\prime}\left(\mathbb{R}^{d} \times \mathbb{R}^{d}\right)$. The associated t-quantization (cf. [135, Sect. 23.3]) to $a, t \in[0,1]$, is defined as the operator $\mathrm{Op}_{t}(a): \mathscr{S}\left(\mathbb{R}^{d}\right) \rightarrow \mathscr{S}^{\prime}\left(\mathbb{R}^{d}\right)$ given by

$$
\left\langle\mathrm{Op}_{t}(a) f, g\right\rangle=\left\langle\mathcal{F}_{\xi \rightarrow(x-y)}^{-1} a((1-t) x+t y, \xi), f \otimes g\right\rangle,
$$

which takes the following form for $a \in \mathscr{S}\left(\mathbb{R}^{d} \times \mathbb{R}^{d}\right)$

$$
\left\langle\mathrm{Op}_{t}(a) f, g\right\rangle=(2 \pi)^{-d} \int e^{i(x-y) \xi} a((1-t) x+t y, \xi) f(y) g(x) d x d y d \xi .
$$

The special cases $t=0, \frac{1}{2}$ and 1 are denoted by $\mathrm{Op}_{0}(a)=: a^{L}(x, D)=: a(x, D)$, $\mathrm{Op}_{1}(a)=: a^{R}(x, D)$ and $\mathrm{Op}_{\frac{1}{2}}(a)=: a^{W}(x, D)$ and are called, left-, right- and Weyl quantization, respectively. 
Remark A.12. For the Weyl quantization of distributions, this may be reformulated in terms of the Wigner distribution function and the pairing (see [61, Prop. 2.5])

$$
\left\langle a^{W}(x, D) f, g\right\rangle=\langle a, \overline{\mathscr{W}(g, \bar{f})}\rangle \quad \forall f, g \in \mathscr{S}\left(\mathbb{R}^{d}\right)
$$

where $\mathscr{W}(f, g) \in \mathscr{S}\left(\mathbb{R}^{2 d}\right)$ is given by

$$
\mathscr{W}(f, g)(x, \xi)=(2 \pi)^{-d} \int_{\mathbb{R}^{d}} f(x+y / 2) \overline{g(x-y / 2)} e^{-i x \xi} d y
$$

and maps $\mathscr{S}\left(\mathbb{R}^{d}\right) \times \mathscr{S}\left(\mathbb{R}^{d}\right) \rightarrow \mathscr{S}\left(\mathbb{R}^{2 d}\right)$.

If $a$ is a symbol in SX, the integral (A.12) is explicitly computable and one obtains the following (cf. [113, Prop. 1.2.7]):

Proposition A.13. Let $a \in \operatorname{SX}\left(\mathbb{R}^{d} \times \mathbb{R}^{d}\right)$. Then for each $t \in[0,1]$ the iterated integral

$$
\mathrm{Op}_{t}(a) f=(2 \pi)^{-d} \int e^{i(x-y) \xi} a((1-t) x+t y, \xi) f(y) d y d \xi
$$

defines a continuous operator $\mathscr{S}\left(\mathbb{R}^{d}\right) \rightarrow \mathscr{S}\left(\mathbb{R}^{d}\right)$, which is extendible by duality ${ }^{69}$ to an operator $\mathscr{S}^{\prime}\left(\mathbb{R}^{d}\right) \rightarrow \mathscr{S}^{\prime}\left(\mathbb{R}^{d}\right)$ for which we use the same notation.

From an operator defined in this way, we can re-obtain its full symbol or amplitude a from $\mathrm{Op}_{0}(a)$ by

$$
a(x, \xi)=e^{i x \xi} \mathrm{Op}_{0}(a) e^{-i x \xi} .
$$

Definition A.14. We write $\operatorname{LSX}^{m}\left(\mathbb{R}^{d}\right)$ for the class of all pseudo-differential operators $A: \mathscr{S}^{\prime}\left(\mathbb{R}^{d}\right) \rightarrow \mathscr{S}^{\prime}\left(\mathbb{R}^{d}\right)$ that can be written in the form $A=\mathrm{Op}_{t}(a)+R$ for some $a \in \mathrm{SX}^{m}\left(\mathbb{R}^{d} \times \mathbb{R}^{d}\right)$ and $R$ a residual element, i.e. a regularizing (or "Schwartzing") continuous operator $R: \mathscr{S}^{\prime}\left(\mathbb{R}^{d}\right) \rightarrow \mathscr{S}\left(\mathbb{R}^{d}\right)$.

Remark A.15. By the Schwartz kernel theorem, residual operators are precisely the ones with a kernel in $\mathscr{S}\left(\mathbb{R}^{d} \times \mathbb{R}^{d}\right)$. Since we have for both symbol classes $\mathscr{S}\left(\mathbb{R}^{d} \times \mathbb{R}^{d}\right)=\bigcap_{r \in \mathbb{R}} \mathrm{SX}^{0-r}\left(\mathbb{R}^{d} \times \mathbb{R}^{d}\right)$, the residual element may be dropped from the definition.

To list further properties of the calculus, we first have to introduce asymptotic expansions. In Section 1.2.4 we consider asymptotic expansions into homogeneous

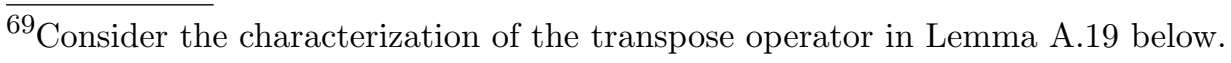


functions. The following definition is valid for general symbols, consider [48, 113, 131 .

Notation (Asymptotic expansions). Let $\left\{m_{j}\right\}_{j \in \mathbb{N}_{0}}$ be a decreasing sequence in $\mathbb{R}$ or $\mathbb{R}^{2}$ such that $\lim _{j \rightarrow \infty} m_{j}=-\infty$ or $(-\infty, \infty)$ respectively. ${ }^{70}$ Let $a \in \mathrm{SX}^{m_{0}}$, $a_{j} \in \mathrm{SX}^{m_{j}}$. Then $a$ is asymptotically equivalent to the asymptotic sum $\sum_{j} a_{j}$, i.e. $a \sim \sum_{j} a_{j}$, if $\forall M \in \mathbb{N}_{0}$ there exists $N \in \mathbb{N}_{0}$ such that $a-\sum_{j=0}^{N} a_{j} \in \mathrm{SX}^{m_{0}-M}$.

Lemma A.16 (Asymptotic completeness). Let $\left\{m_{j}\right\}_{j \in \mathbb{N}_{0}}$ be a decreasing sequence in $\mathbb{R}$ or $\mathbb{R}^{2}$ as above, $a_{j} \in \mathrm{SX}^{m_{j}}$. Then there exists $a \in \mathrm{SX}^{m_{0}}$, determined uniquely up to elements in $\mathscr{S}\left(\mathbb{R}^{2 d}\right)$, such that $a \sim \sum_{j} a_{j}$.

For a proof by approximation, using cut-offs, consider e.g. [36, Lem. 1.6.4], [113, Prop. 1.1.6], [131, Thm. 1.4.3], [135, Prop. 23.1].

Different quantizations may be related in terms of a certain Fourier multiplier or asymptotic expansions of the respective symbols (cf. [113, Thm. 1.2.4, Prop. 1.2.5 and 1.2.12]):

Proposition A.17 (Change of quantization). Using the Fourier multiplier

$$
e^{i\left(t_{1}-t_{2}\right) D_{x} D_{\xi}}: \mathrm{SX}^{m} \rightarrow \mathrm{SX}^{m}
$$

defined as a continuous map in terms of the oscillatory integral

$$
e^{i t D_{x} D_{\xi}} a(x, \xi)=(2 \pi)^{-2 d} \int e^{i(x y+\xi \eta)} e^{i t y \eta} \mathcal{F} a(y, \eta) d y d \eta
$$

we have

$$
\mathrm{Op}_{t_{1}}(a)=\mathrm{Op}_{t_{2}}\left(e^{i\left(t_{1}-t_{2}\right) D_{x} D_{\xi}} a\right)
$$

Furthermore, the following asymptotic expansion holds:

$$
e^{i t D_{x} D_{\xi}} a(x, \xi) \sim \sum_{\alpha} \frac{(i t)^{|\alpha|}}{\alpha !} D_{\xi}^{\alpha} D_{x}^{\alpha} a(x, \xi) .
$$

In particular, $e^{i t D_{x} D_{\xi}} a(x, \xi)$ is elliptic at $\left(x_{0}, \xi_{0}\right)$ if and only if $a(x, \xi)$ is elliptic at $\left(x_{0}, \xi_{0}\right)$.

Remark A.18. Using this result, we can re-obtain $a$ from $\mathrm{Op}_{t}(a)$ for any $t \in[0,1]$ using A.15 and Proposition A.17.

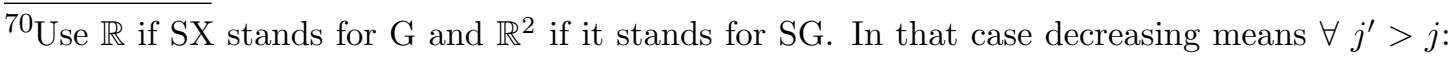
$\left(m_{e}, m_{\psi}\right)_{j^{\prime}}-\left(m_{e}, m_{\psi}\right)_{j} \in\left((-\infty, 0]^{2} \backslash(0,0)\right)$.
} 
Furthermore, this may be used to obtain information on adjoints and transposes, cf. e.g. [113, Prop. 1.2.12].

Lemma A.19 (Characterization of the transpose operator). Let $a \in \mathrm{SX}^{m}$. Then ${ }^{t} \mathrm{Op}_{t}(a)=\mathrm{Op}_{1-t}(a(\cdot,-\cdot))$. In particular, its symbol is

$$
b(x, \xi)=e^{i(1-2 t) D_{x} D_{\xi}} a(x,-\xi)
$$

and ${ }^{t} \mathrm{Op}_{t}(a)$ is SG-elliptic at $\left(x_{0},-\xi_{0}\right)$ if and only if $\mathrm{Op}_{t}(a)$ is SG-elliptic at $\left(x_{0}, \xi_{0}\right)$.

The symbol of the adjoint operator is given via

$$
b(x, \xi)=e^{i(1-2 t) D_{x} D_{\xi}} \bar{a}(x, \xi) .
$$

For either object, A.17 may be used to obtain an asymptotic expansion.

The same map may be used to describe what happens under composition of operators ([113, Thm. 1.2.16]):

Lemma A.20 (Composition of operators). Let $a_{1} \in \mathrm{SX}^{m_{1}}$ and $a_{2} \in \mathrm{SX}^{m_{2}}$. Then $\mathrm{Op}_{1}\left(a_{1}\right) \mathrm{Op}_{1}\left(a_{2}\right)=\mathrm{Op}_{1}(b)$ with $b \in \mathrm{SX}^{m_{1}+m_{2}}$ given by

$$
b(x, \xi)=\left.e^{i D_{y} D_{\eta}} a_{1}(x, \eta) a_{2}(y, \xi)\right|_{(x, \eta)=(y, \xi)} .
$$

In principle, using Proposition A.17, composition formulae for any t-quantization may be obtained. For the Weyl quantization it takes the simple form (see [113, Thm. 1.2.17] and [83, Formula 18.5.6]):

$$
\begin{aligned}
a_{1}^{W}(x, D) a_{2}^{W}(x, D) & =b^{W}(x, D), \\
b(x, \xi) & =\left.e^{\frac{i}{2} \omega\left(D_{x}, D_{y} ; D_{\eta}, D_{\xi}\right)} a_{1}(x, \eta) a_{2}(y, \xi)\right|_{(x, \eta)=(y, \xi)}, \\
b(x, \xi) & \sim \sum_{\alpha, \beta} \frac{(-1)^{|\beta|}}{2^{-|\alpha|-|\beta|} \alpha ! \beta !} \partial_{\xi}^{\alpha} D_{x}^{\beta} a_{1}(x, \xi) \partial_{\xi}^{\beta} D_{x}^{\alpha} a_{2}(x, \xi) .
\end{aligned}
$$

Having discussed compositions, it is possible to introduce a quasi-inverse or parametrix of a pseudodifferential operator, cf. [113, Thm. 1.3.6].

Theorem A.21 (Existence of a parametrix). Let $a \in \mathrm{SX}^{m}$ be elliptic, $t \in[0,1]$. Then there exists $b \in \mathrm{SX}^{-m}$ and regularizing operators $R_{1}, R_{2}$ such that

$$
\mathrm{Op}_{t}(a) \mathrm{Op}_{t}(b)=\mathrm{id}+R_{1} \quad \mathrm{Op}_{t}(b) \mathrm{Op}_{t}(a)=\mathrm{id}+R_{2}
$$


as operators on $\mathscr{S}\left(\mathbb{R}^{d}\right)$ as well as on $\mathscr{S}^{\prime}\left(\mathbb{R}^{d}\right)$. The operator $\mathrm{Op}_{t}(b)$ is called a parametrix for $\mathrm{Op}_{t}(a)$ and is unique modulo regularizing operators.

If a is classical, then $b$ may be chosen classical as well.

When dealing with localized phenomena in $T^{*} \mathbb{R}^{d}$, it is often necessary to consider a localized version of this theorem, consider [36, Thm. 2.3.3], [94, Thm. 1.1.15.].

Definition A.22. A symbol $a \in \mathrm{SX}^{m}$ is called SX-elliptic with respect to $\phi \in \mathrm{SX}^{0}$, if $a$ is elliptic on all of $\operatorname{Csp}(\phi) \cap W_{\mathrm{SX}}$.

Theorem A.23 (Existence of a local parametrix). Let $a \in \mathrm{SX}^{m}$ SX-elliptic with respect to $\phi \in \mathrm{SX}^{0}$. Then there exists $b_{j} \in \mathrm{SX}^{-m}, j \in\{1,2\}$ and regularizing operators $R_{j}: \mathscr{S}^{\prime}\left(\mathbb{R}^{d}\right) \rightarrow \mathscr{S}\left(\mathbb{R}^{d}\right)$ such that

$$
a(x, D) b_{1}(x, D)=\phi(x, D)+R_{1} \quad b_{2}(x, D) a(x, D)=\mathrm{Op}_{1}(\phi)+R_{2} .
$$

Additionally, there exists $b_{j} \in \mathrm{SX}^{-m}, j \in\{1,2\}$ and regularizing operators $R_{j}^{\prime}$ such that

$$
a^{W}(x, D) b_{1}^{W}(x, D)=\phi^{W}(x, D)+R_{1}^{\prime} \quad b_{2}^{W}(x, D) a^{W}(x, D)=\phi^{W}(x, D)+R_{2}^{\prime} .
$$

The next section is devoted to spaces that are specifically adapted for pseudodifferential analysis in terms of a given calculus, the so-called Sobolev spaces.

\section{A.3.2. Sobolev spaces and $L^{2}$-boundedness.}

Global Sobolev spaces intermediate spaces between $\mathscr{S}^{\prime}$ and $\mathscr{S}$ that provide a resolution of $\mathscr{S}^{\prime}$ in terms of continuous parameters, thus allowing us to study the mapping properties of $\Psi$ DOs in a more refined way. For the two calculi independently, they are discussed in detail in [131, Sect. 1.4.2] and [135, Sect. 25]. We introduce them on the basis of [113] ${ }^{71}$ cf. also [94, Sect. 2.6]. In order to do so, it is first important to study the action of $\Psi$ DOs on $L^{2}\left(\mathbb{R}^{d}\right)$ (see [113, Thm. 1.4.1]):

Theorem A.24 (Calderón-Vaillancourt). Let a an element of $\mathrm{SG}^{0,0}\left(\mathbb{R}^{d} \times \mathbb{R}^{d}\right)$ or $\mathrm{G}^{0}\left(\mathbb{R}^{d} \times \mathbb{R}^{d}\right)$. Then the corresponding quantization $a(x, D)$ is bounded on $L^{2}\left(\mathbb{R}^{d}\right)$ and we have the estimate

$$
\|a(x, D)\|_{\mathscr{B}\left(L^{2}\left(\mathbb{R}^{d}\right)\right)} \lesssim \rho_{N}^{0,0}(a),
$$

\footnotetext{
${ }^{71}$ We list the particularly relevant sections: for the G-case consider Chapter 1.5, Chapter 1.7.4, Definition 2.1.8 and the following discussion and for the SG-case Definition 3.1.4 and the following discussion.
} 
or correspondingly

$$
\|a(x, D)\|_{\mathscr{B}\left(L^{2}\left(\mathbb{R}^{d}\right)\right)} \lesssim \rho_{N}^{0}(a),
$$

where $N \in \mathbb{N}_{0}$ depends only on the dimension.

Definition A.25 (Sobolev spaces). Let $A \in \mathrm{SX}^{m}\left(\mathbb{R}^{d} \times \mathbb{R}^{d}\right)$ be SX-elliptic. Denote by $B$ a corresponding parametrix (see Theorem A.21) and by $R$ the residual element that satisfies $B A+R=\mathrm{id}$.

The Sobolev space $\mathrm{H}_{\mathrm{SX}}^{m}\left(\mathbb{R}^{d}\right)$ (i.e. $\mathrm{H}_{\mathrm{SG}}^{m_{e}, m_{\psi}}\left(\mathbb{R}^{d}\right)$ or $\mathrm{H}_{\mathrm{G}}^{m}\left(\mathbb{R}^{d}\right)$ ) of index $m$ is defined as the normed space

$$
\mathrm{H}_{\mathrm{SX}}^{m}\left(\mathbb{R}^{d}\right):=\left\{u \in \mathscr{S}^{\prime}\left(\mathbb{R}^{d}\right) \mid A u \in L^{2}\left(\mathbb{R}^{d}\right)\right\}
$$

with the norm

$$
\|u\|_{\mathrm{H}_{\mathrm{SX}}^{m}\left(\mathbb{R}^{d}\right)}=\|A u\|_{L^{2}\left(\mathbb{R}^{d}\right)}+\|R u\|_{L^{2}\left(\mathbb{R}^{d}\right)} .
$$

Example A.26. In the simplest case, one may use the left quantizations of $\langle x\rangle^{m_{x}}\langle\xi\rangle^{m_{\xi}}$ in the SG- and $\langle(x, \xi)\rangle^{m}$ in G-case to introduce these spaces. Both operators are exactly invertible, thus we can neglect the $R$-term in the norm.

We list some well-known properties of Sobolev spaces ${ }^{72}$

Proposition A.27 (Properties of Sobolev spaces).

- $\mathrm{H}_{\mathrm{SX}}^{m}\left(\mathbb{R}^{d}\right)$ does not depend on the choice of $(A, B, R)$, meaning that different choices yield the same space endowed with an equivalent norm.

- Let $A^{\prime} \in \operatorname{LSX}^{m^{\prime}}\left(\mathbb{R}^{d} \times \mathbb{R}^{d}\right)$. Then $\forall m \in \mathbb{R}$ or $\left(m_{e}, m_{\psi}\right) \in \mathbb{R}^{2}$ we have a continuous extension of $A^{\prime}$, denoted by the same letter, mapping $\mathrm{H}_{\mathrm{SX}}^{m}\left(\mathbb{R}^{d}\right)$ continuously into $\mathrm{H}_{\mathrm{SX}}^{m-m^{\prime}}\left(\mathbb{R}^{d}\right)$. If $A^{\prime}$ is elliptic, then it its extension constitutes a Fredholm operator.

- We have the continuous inclusions $\mathscr{S}\left(\mathbb{R}^{d}\right) \hookrightarrow \mathrm{H}_{\mathrm{SX}}^{m}\left(\mathbb{R}^{d}\right) \hookrightarrow \mathscr{S}^{\prime}\left(\mathbb{R}^{d}\right)$. In fact we have

$$
\bigcap_{m} \mathrm{H}_{\mathrm{SX}}^{m}\left(\mathbb{R}^{d}\right)=\mathscr{S}\left(\mathbb{R}^{d}\right) \quad \bigcup_{m} \mathrm{H}_{\mathrm{SX}}^{m}\left(\mathbb{R}^{d}\right)=\mathscr{S}^{\prime}\left(\mathbb{R}^{d}\right),
$$

where the index $m$ runs over all possible choices of $m \in \mathbb{R}$ or $\left(m_{e}, m_{\psi}\right) \in \mathbb{R}^{2}$ respectively.

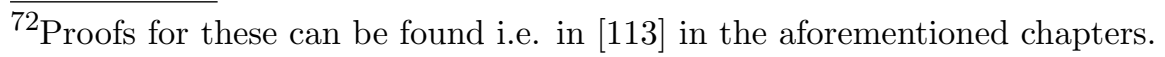


For the special case of the SG-calculus and $\left(m_{e}, m_{\psi}\right) \in \mathbb{N}_{0}^{2}$, we have the equivalent definition, with an equivalent norm

$$
\begin{aligned}
\mathrm{H}_{\mathrm{SG}}^{m_{e}, m_{\psi}}\left(\mathbb{R}^{d}\right) & =\left\{u \in \mathscr{S}^{\prime}\left(\mathbb{R}^{d}\right) \mid x^{\alpha} D^{\beta} u \in L^{2}\left(\mathbb{R}^{d}\right) \text { for }|\alpha| \leq m_{e},|\beta| \leq m_{\psi}\right\}, \\
\|u\|_{\mathrm{H}_{\mathrm{SG}}}^{*} m_{e}, m_{\psi} & =\sum_{\substack{|\alpha| \leq m_{e},|\beta| \leq m_{e}}}\left\|x^{\alpha} D^{\beta} u\right\|_{L^{2} .}
\end{aligned}
$$

Notice thus that $\mathrm{H}_{\mathrm{SG}}^{0, m_{\psi}}\left(\mathbb{R}^{d}\right)$ coincides with the classical Sobolev space, denoted here by $\mathrm{H}_{\mathrm{cl}}^{m_{\psi}}\left(\mathbb{R}^{d}\right)$ and thus we have $\mathrm{H}_{\mathrm{SG}}^{m_{e}, m_{\psi}}\left(\mathbb{R}^{d}\right)=\langle x\rangle^{m_{e}} \mathrm{H}_{\mathrm{cl}}^{m_{\psi}}\left(\mathbb{R}^{d}\right)$, i.e. the SG-sobolev space is a weighted version of the standard classical Sobolev space.

For the special case of the G-calculus, we have if $m \in \mathbb{N}_{0}$ the equivalent definition, with an equivalent norm

$$
\begin{aligned}
\mathrm{H}_{\mathrm{G}}^{m}\left(\mathbb{R}^{d}\right) & =\left\{u \in \mathscr{S}^{\prime}\left(\mathbb{R}^{d}\right) \mid x^{\alpha} D^{\beta} u \in L^{2}\left(\mathbb{R}^{d}\right) \text { for }|\alpha|+|\beta| \leq m\right\}, \\
\|u\|_{\mathrm{H}_{\mathrm{G}}^{m}}^{*} & =\sum_{|\alpha|+|\beta| \leq m}\left\|x^{\alpha} D^{\beta} u\right\|_{L^{2}} .
\end{aligned}
$$

We remark that both Sobolev spaces may be obtained as special cases of modulation spaces. We follow [68, Chap. 11] to introduce these and restrict our analysis to a subclass of these spaces useful for the analysis of tempered distributions.

Modulation spaces are designed specifically for applicability in time-frequency analysis and were invented by Feichtinger (consider [60] for a broad discussion about the rich history and applications of these spaces). Due to their wellunderstood behaviour with respect to the analytical tools of time-frequency analysis they have been used to great extent in microlocal analysis, consider e.g. [31, the series of articles [40, 41, 42, 43] as well as [68, Chap. 11] and the references therein.

Definition A.28 (Modulation spaces). Let $w(x, \xi)$ a non-negative weight function satisfying

- $w \in L_{\text {loc }}^{1}\left(\mathbb{R}^{2 d}\right)$,

- $\left|w\left(x_{1}+x_{2}, \xi_{1}+\xi_{2}\right)\right| \lesssim w\left(x_{1}, \xi_{1}\right)\left\langle\left(x_{2}, \xi_{2}\right)\right\rangle^{s}$ for some $s \geq 0$. 
The Modulation space $\mathscr{M}_{w}^{p, q}\left(\mathbb{R}^{d}\right)$ consists of all $u \in \mathscr{S}^{\prime}\left(\mathbb{R}^{d}\right)$ such that for some non-zero window $g \in \mathscr{S}\left(\mathbb{R}^{d}\right) \mathscr{V}_{g} u \in L_{m}^{p, q}\left(\mathbb{R}^{2 d}\right)$, i.e.

$$
\left\|\mathscr{V}_{g} u\right\|_{L_{m}^{p, q}\left(\mathbb{R}^{2 d}\right)}=\left(\int\left(\int w(x, \xi)^{p}\left|\mathscr{V}_{g} u(x, \xi)\right|^{p} d x\right)^{\frac{q}{p}} d \xi\right)^{\frac{1}{q}}<\infty .
$$

We obtain the previous Sobolev spaces by choosing the weights as follows (cf. [42],68, Prop. 11.3.1]):

- If $w=\langle(x, \xi)\rangle^{m}$, we obtain $\mathscr{M}_{w}^{2,2}\left(\mathbb{R}^{d}\right)=\mathrm{H}_{\mathrm{G}}^{m}\left(\mathbb{R}^{d}\right)$,

- If $w=\langle x\rangle^{m_{e}}\langle\xi\rangle^{m_{\xi}}$, we obtain $\mathscr{M}_{w}^{2,2}\left(\mathbb{R}^{d}\right)=\mathrm{H}_{\mathrm{SG}}^{m_{e}, m_{\psi}}\left(\mathbb{R}^{d}\right)$. 


\section{A.3.3. The action of pseudo-differential operators and the STFT.}

The symbol of a pseudo-differential operator may be regarded as a function of $T^{*} \mathbb{R}^{d}$. It is therefore natural to examine its relationship to the STFT - i.e. to understand how the action of a pseudo-differential operator on a distribution can be understood in the time-frequency plane.

For that, we first introduce a class of operators defined in terms of the STFT of a distribution. They are known under the name of Anti-Wick quantized operators, localization operators, Toeplitz operators and many others, depending on the context. For an introduction from the viewpoint of time-frequency analysis and additional resources on this rich topic we refer to [28] and [35] and the references therein.

Definition A.29 (Localization operator). Let $a \in \mathscr{S}^{\prime}\left(\mathbb{R}^{2 d}\right), g \in \mathscr{S}\left(\mathbb{R}^{d}\right) \backslash\{0\}$ a window function. Then the localization operator $A_{a}^{g}: \mathscr{S}\left(\mathbb{R}^{d}\right) \rightarrow \mathscr{S}^{\prime}\left(\mathbb{R}^{d}\right)$ is given by

$$
A_{a}^{g} u=\mathscr{V}_{g}^{*}\left(a \mathscr{V}_{g} u\right)
$$

or equivalently, in the weak formulation,

$$
\left\langle A_{a}^{g} u, \bar{v}\right\rangle=\left\langle a, \mathscr{V}_{g} u \overline{\mathscr{V}_{g} v}\right\rangle .
$$

The following proposition (see e.g. [113, Prop. 1.7.9], [135, Thm. 24.1]) allows us to rewrite a localization operator with standard window in terms of a Weylquantized pseudo-differential operator.

Proposition A.30 (Weyl-Wick connection). Let $a \in \mathscr{S}^{\prime}\left(\mathbb{R}^{2 d}\right)$. Then $A_{a}^{\psi_{0}}=b^{W}$, where $b \in \mathscr{S}^{\prime}\left(\mathbb{R}^{2 d}\right)$ is given by

$$
b(x, \xi)=\pi^{-d}\left(a * e^{-|\cdot|^{2}}\right)(x, \xi) .
$$

To now relate how pseudo-differential operators act on the STFT of a distribution, we wish to write for $a \in \mathrm{SX}$

$$
\mathscr{V}_{\psi_{0}} a(x, D) u=A \mathscr{V}_{\psi_{0}} u
$$

for some operator $A: \mathscr{S}^{\prime}\left(\mathbb{R}^{2 d}\right) \rightarrow \mathscr{S}^{\prime}\left(\mathbb{R}^{2 d}\right)$. From the Moyal identity (A.8) we can deduce $\mathscr{V}_{\psi_{0}} a(x, D) u=\mathscr{V}_{\psi_{0}} a(x, D) \mathscr{V}_{\psi_{0}}^{*} \mathscr{V}_{\psi_{0}} u$ and thus $A=\mathscr{V}_{\psi_{0}} a(x, D) \mathscr{V}_{\psi_{0}}^{*}$ Its Schwartz kernel $\mathcal{K}_{A}$ can be explicitly computed (consider [113, Eq. (1.7.27)]) 
to equal the oscillatory integral

$$
\mathcal{K}_{A}\left(y^{\prime}, \eta^{\prime} ; y, \eta\right)=(2 \pi)^{-2 d} \int e^{i x\left(\eta-\eta^{\prime}\right)+i \xi\left(y^{\prime}-y\right)+i x \xi} a\left(x+y^{\prime}, \xi+\eta\right) \psi_{0}(\xi) \psi_{0}(x) d x d \xi
$$

By the same reasoning as in [113], we can deduce for arbitrary $N \in \mathbb{N}_{0}$ the estimate

$$
\left|\mathcal{K}_{A}\left(y^{\prime}, \eta^{\prime} ; y, \eta\right)\right| \lesssim C_{a, N}\left\langle y-y^{\prime}\right\rangle^{-2 N}\left\langle\eta-\eta^{\prime}\right\rangle^{-2 N},
$$

where $C_{a, N}$ can be given by some semi-norm $\|a\|_{\mathrm{SX}^{m(N)}}$. We therefore have for any $N \in \mathbb{N}_{0}$

$$
\left|\mathscr{V}_{\psi_{0}} a(x, D) u\right| \lesssim C_{a, N}\left(\left(\left\langle x^{\prime}\right\rangle^{-2 N}\left\langle\xi^{\prime}\right\rangle^{-2 N}\right) *\left|\mathscr{V}_{\psi_{0}} u\right|\right)
$$

To sum up: we may estimate the STFT of a pseudo-differential operator acting on $u$ in terms of a convolution of $\mathscr{V}_{\psi_{0}} u$ with arbitrary high negative powers of the weights $\langle x\rangle\langle\xi\rangle$ and we may rewrite the action of a localization operator in terms of a pseudo-differential operator, which involve a convolution by a (rapidly decaying) Gaussian. It is therefore necessary be able to estimate such convolutions, cf. e.g. [121, Prop. 2.2].

Lemma A.31. Suppose $F$ is a measurable function that satisfies

$$
|F(x)| \lesssim\langle x\rangle^{M} \text { for some } M \in \mathbb{N}_{0},
$$

suppose $x_{0} \in \mathbb{S}^{d-1}$ and $x_{0} \in \Gamma$ for some open conic set $\Gamma \subset \mathbb{R}^{d} \backslash\{0\}$. Suppose further that $F$ is rapidly decaying on $\Gamma$, i.e.

$$
\forall N \in \mathbb{N}_{0}:|F|_{\Gamma}(x) \mid \lesssim\langle x\rangle^{-N}
$$

Let $f \in \mathscr{S}\left(\mathbb{R}^{d}\right)$. Then $f * F$ is a smooth and polynomially bounded function, which is rapidly decaying on all open subcones $\Gamma^{\prime} \subset \mathbb{R}^{d} \backslash\{0\}$ containing $x_{0}$ such that $\overline{\Gamma^{\prime}} \subset \Gamma$. 
Appendix B. List of RECURRING SYMBOLS AND ABBREVIATIONS

Notation: Roman letters

\begin{tabular}{|c|c|c|}
\hline Symbol & Description & Reference \\
\hline$a, b$ & Amplitude functions/Symbols & Sect. 1.2 \\
\hline$a^{\bullet}(x, D)$ & Various quantizations of $a$ (with $\bullet \in\{L, R, W\}$ ) & Sect. 1.2 \\
\hline$A_{a}^{g}$ & ocalization operator with symbol $a$ and window $g$ & Def. A.29 \\
\hline $\mathbb{B}^{d}$ & Unit ball, radial compactification of $\mathbb{R}^{d}$ & Sect. 1.1 .1 \\
\hline $\mathcal{C}_{\varphi}$ & Critical set of $\varphi$ w.r.t. the second variable & Def. 3.14 \\
\hline Css, Csp & one (singular) support of a tempered distribution & $(1.6)$ \\
\hline $\mathscr{C}^{\infty}(X)$ & Smooth functions on $X$, up to $\partial X$ if present & \\
\hline$\dot{\mathscr{C}}^{\infty}(X)$ & $\mathscr{C}^{\infty}$-functions vanishing of order $\infty$ on $\partial X$ & Sect. 1.1 .1 \\
\hline $\mathcal{D}_{\lambda}, \mathcal{D}_{A}$ & Dilation by $\lambda>0$ or $A \in \mathrm{GL}(d, \mathbb{R})$ & Def. A.5 \\
\hline $\mathcal{F}, \widehat{u}$ & Fourier transform $($ of $\mathrm{u})$ & Sect. A.1 \\
\hline $\mathscr{F}_{\lambda}, \mathscr{F}_{\lambda, \mu}$ & (Generalized) Fourier-Bros-Iagolnitzer transform & (A.6);(2.7) \\
\hline $\mathrm{G}$ & Shubin G-symbols & Def. 1.12 \\
\hline $\mathrm{GL}(d, \mathbb{R})$ & Group of invertible $\mathbb{R}$-valued $d \times d$-matrices & \\
\hline $\mathrm{H}_{\mathrm{SG}}, \mathrm{H}_{\mathrm{G}}$ & Adapted Sobolev spaces to the SG and G calculi & Sect. A.3.2 \\
\hline$I_{\varphi}(a)$ & Oscillatory integral with phase $\varphi$ and symbol $a$ & Thm. 3.9 \\
\hline $\mathcal{K}_{A}$ & Schwartz kernel of the operator $A$ & Thm. A.4 \\
\hline LG & The class of G- $\Psi$ DOs & Def. A.14 \\
\hline $\mathrm{LSG}_{(\mathrm{cl})}$ & The class of (classical) SG- $\Psi$ DOs & Def. A.14 \\
\hline $\mathbb{M}^{1, d}$ & Minkowski space & Sect. 3.5.2 \\
\hline $\mathcal{M}_{\xi_{0}}$ & Modulation by $\xi_{0} \in \mathbb{R}^{d}$ & Def. A.5 \\
\hline$m, m_{\bullet}$ & $\begin{array}{l}\text { Symbol orders, in Section } 3.5: \text { mass } \\
\end{array}$ & \\
\hline$n_{e}, n_{\psi}$ & Phase function order & \\
\hline $\mathrm{Op}_{t}(a)$ & $t$-quantization of the symbol $a$ & Sect. 1.2 \\
\hline $\operatorname{pr}_{\bullet}, \operatorname{pr}_{j}$ & Projection on $\bullet /$ the $j$ th set of variables & \\
\hline $\operatorname{sd}_{X}^{\perp}(u)$ & Transversal scaling degree of $u$ at $X$ & Def. 3.43 \\
\hline $\mathrm{S}_{(\mathrm{cl})}$ & (polyhomogeneous) Symbols in one variable & Sect. 1.2 .1 \\
\hline $\mathrm{SG}_{(\mathrm{cl})}$ & (polyhomogeneous) SG-symbols & Def. 1.12 \\
\hline $\mathscr{S}, \mathscr{S}^{\prime}$ & Schwartz functions, Tempered distributions & Def. A.1 \\
\hline $\mathscr{S}_{\theta}, \mathscr{S}_{\theta}^{\prime}$ & Gelfand-Shilov functions and ultradistributions & Def. 4.1 \\
\hline $\mathbb{S}^{d-1}$ & $d-1$-dimensional sphere & \\
\hline $\mathcal{T}_{x_{0}}$ & Translation by $x_{0} \in \mathbb{R}^{d}$ & Def. A.5 \\
\hline $\mathscr{V}_{g}$ & Short-time Fourier transform with window $g$ & Def. A.7 \\
\hline $\mathrm{W}_{\mathrm{G}}$ & G-wave front space & Sect. 1.1 .4 \\
\hline $\mathrm{W}_{\mathrm{SG}}$ & SG-wave front space & Sect. 1.1 .4 \\
\hline $\mathrm{WF}_{\mathrm{G}}$ & G-wave front set & Def. 2.55 \\
\hline $\mathrm{WF}_{\mathrm{SG}}$ & SG-wave front set & Def. 2.3 \\
\hline
\end{tabular}


Notation: Greek letters

\begin{tabular}{|c|c|c|}
\hline Symbol & Description & Reference \\
\hline$\alpha^{e}$ & 1-form associated to $\varrho^{\psi}$ & Def. 3.29 \\
\hline$\alpha^{\psi}$ & Canonical 1-form & \\
\hline$\delta_{x_{0}}$ & Dirac delta distribution at $x_{0}$ & \\
\hline$\Delta_{+}$ & Two-point function & Def. 3.65 \\
\hline$\Gamma, \Gamma(U)$ & Cones in $\mathbb{R}^{d}$, Conification of $U \subset \mathbb{S}^{d-1}$ & Const. 1.1 \\
\hline$\iota$ & Embedding $\mathbb{R}^{d} \rightarrow\left(\mathbb{B}^{d}\right)^{o}$ & Sec. $1 . \overline{1.1}$ \\
\hline$\Lambda, \Lambda_{\varphi}$ & Lagrangian submanifold (associated to $\varphi$ ) & Def. 3.17 \\
\hline$\rho_{N}^{\mathscr{S}}, \rho_{N, M}^{\mathscr{S}}$ & Semi-norms on the Schwartz functions & $(\mathrm{A} .2),(\mathrm{A} .3)$ \\
\hline$\rho_{N}^{m_{e}, m_{\psi}}$ & Semi-norm on $\mathrm{SG}^{m_{e}, m_{\psi}}$ & Sect. 1.2 .3 \\
\hline$\rho_{N}^{m}$ & Semi-norm on $\mathrm{G}^{m}\left(\right.$ and $\left.S^{m}\right)$ & Sect. $\overline{1.2 .1}$ \\
\hline$\varrho^{e}$ & Exit radial vector field & Def. 3.29 \\
\hline$\varrho^{\psi}$ & Radial vector field & $(3.31)$ \\
\hline$\sigma_{\bullet}^{\bullet}$ & Principal symbols associated to $\Psi \mathrm{DO}$ & Def. 1.17 \\
\hline$\phi^{x_{0}}, \phi_{R}^{x_{0}}$ & Cut-off (around a point $\left.x_{0}\right)$ & Const. 1.3 \\
\hline$\varphi$ & Phase function & Def 3.7 \\
\hline$\chi$ & Excision function & Sect. 1.1 .2 \\
\hline$\psi_{0}, \psi_{k}$ & Normalized and shifted Gaussians & Def. A.7: 2.14 \\
\hline$\omega$ & Canonical 2-form & Sect.A.1 \\
\hline$\omega_{m}$ & energy-momentum relation & Sect. 3.5.2 \\
\hline
\end{tabular}

\section{Notation: Mathematical symbols}

\begin{tabular}{|c|c|c|}
\hline Symbol & Description & Reference \\
\hline$\langle x\rangle$ & Japanese bracket, $x \mapsto \sqrt{1+|x|^{2}}$ & Sect. A.1 \\
\hline$f \lesssim g$ & $\exists C>0$ such that $\forall x \in X: f(x) \leq C g(x)$ & Sect. A.1 \\
\hline$f \asymp g$ & Asymptotical equivalence: $f \lesssim g, g \lesssim f$ & Sect. A.1 \\
\hline$\tilde{U}$ & Set in $\mathbb{B}^{d}$ associated to $U \subset \mathbb{R}^{d}$ & Const. 1.1 \\
\hline$x_{0} \infty$ & Point "at infinity" associated to $x_{0}$ & Const. $\overline{1.1}$ \\
\hline$x_{1} \sim x_{2}$ & $x_{1}=\lambda x_{2}$ for $\lambda>0$ & Const. $\overline{1.1}$ \\
\hline$a \sim \sum_{j=0}^{\infty} a_{j}$ & Asymptotic equivalence & Sect. $1 . \overline{2.4}$ \\
\hline
\end{tabular}




\section{Abbreviations}

\begin{tabular}{|c|c|}
\hline Abbreviation & Description \\
\hline App. & Appendix \\
\hline cf. & Confer; compare to \\
\hline Chap. & Chapter \\
\hline Def. & Definition \\
\hline e.g. & Exempli gratia, for example \\
\hline etc. & Et cetera; and so forth \\
\hline FBI & Fourier-Bros-Iagolnitzer \\
\hline Fig. & Figure \\
\hline i.e. & Id est; that is \\
\hline Lem. & Lemma \\
\hline Prop. & Proposition \\
\hline IDO & Pseudo-differential operator \\
\hline QFT & Quantum field theory \\
\hline Rem. & Remark \\
\hline Sect. & Section \\
\hline STFT & Short-time Fourier transform \\
\hline s.t. & Such that \\
\hline Thm. & Theorem \\
\hline w.l.o.g. & Without loss of generality \\
\hline w.r.t. & With respect to \\
\hline
\end{tabular}




\section{REFERENCES}

[1] G.D. Andrews, SG Fourier Integral Operators with Closure under Composition, Diss. Imperial College London, 2009.

[2] K. Asada, On the $L^{2}$-Boundedness of Fourier Integral Operators in $\mathbb{R}^{n}$, Proc. Japan Acad. 57 Ser. A (1981).

[3] K. Asada, D. Fujiwara, On some oscillatory integral transformations in $L^{2}\left(\mathbb{R}^{n}\right)$, Japan. J. Math. (N.S.) 4 (1978), 299-361.

[4] D. Bahns, The ultraviolet infrared mixing problem on the noncommutative Moyal space, preprint, arXiv:1012.3707 (2010).

[5] D. Bahns, Perturbative methods on the noncommutative Minkowski space, DESY-THESIS2004-004, 2003.

[6] D. Bahns and M. Wrochna, On-shell extension of distributions, Annales Henri Poincaré Online first (2013), DOI:10.1007/s00023-013-0288-y.

[7] U. Battisti, S. Coriasco and E. Schrohe, Fourier Integral Operators of Boutet de Monvel Type, preprint, arXiv:1407.2738 (2014).

[8] U. Battisti, S. Coriasco and E. Schrohe, On a Class of Fourier Integral Operators on Manifolds with Boundary, preprint, arXiv:1406.0636 (2014).

[9] R. Beals and C. Fefferman, Classes of Spatially Inhomogeneous Pseudodifferential Operators, Proc. Nat. Acad. Sci. USA 705 (1973), 1500-1501.

[10] G. Bengel, Wave Front Sets and Singular Supports of Convolutions, Publ. Res. Inst. Math. Sci. 1299 (1976), 1-4.

[11] N. N. Bogoliubov, D. V. Shirkov, Introduction to the theory of quantized fields, J. Wiley, New York, 1980.

[12] J.M. Bony, Equivalence des diverses notions de spectre singulier analytique, Séminaire Goulaouic-Schwartz, exp. n. 3 (1976-77), 1-12.

[13] J.M. Bony, Second Microlocalization and Propagation of Singularities for Semi-Linear Hyperbolic Equations, Hyperbolic Equations and Related Topics, (Katata/Kyoto, 1984), Academic Press, Boston, MA, 1986, 11-49.

[14] M. Borsero and R. Schulz, Microlocal properties of bisingular operators, J. Pseudo-Differ. Oper. Appl. 5 (2014), 43-67, DOI 10.1007/s11868-013-0083-z

[15] J. Bros and D. Iagolnitzer, Support essentiel et structure analytique des distributions, Séminaire Goulaouic-Meyer-Schwartz, exp. 18 (1975-76).

[16] R. Brunetti and K. Fredenhagen, Microlocal Analysis and Interacting Quantum Field Theories: Renormalization on Physical Backgrounds, Commun. Math. Phys. 2083 (2000), 623-661.

[17] P. Blanchard and R. Sénéor, Green's functions for theories with massless particles (in perturbation theory), Ann. Inst. Poincaré 232 (1975), 147-209.

[18] M. Cappiello, Fourier integral operators and Gelfand-Shilov spaces, Oper. Theory Adv. Appl. 160 (2005), 81-100.

[19] M. Cappiello, Fourier integral operators of infinite order and applications to SG-hyperbolic equations, Tsukuba J. Math. 282 (2004), 311-362.

[20] M. Cappiello, T. Gramchev and L. Rodino, Gelfand-Shilov spaces, pseudo-differential operators and localization operators, Oper. Theory Adv. Appl. 172 (2007), 297-312.

[21] M. Cappiello and L. Rodino, SG-pseudodifferential operators and Gelfand-Shilov spaces, Rocky Mountain J. Math. 364 (2006), 1117-1148.

[22] M. Cappiello and R. Schulz, Microlocal analysis of quasianalytic Gelfand-Shilov type ultradistributions, preprint, arXiv:1309.4236 (2013). 
[23] S.Y. Chung and D. Kim, A quasianalytic singular spectrum with respect to the DenjoyCarleman class, Nagoya Math. J. 148 (1997), 137-149.

[24] J. Chung, S. Y. Chung and D. Kim, Characterization of the Gelfand-Shilov spaces via Fourier transforms, Proc. Am. Math. Soc. 1247 (1996), 2101-2108.

[25] J.F. Colombeau, Multiplication of distributions, B. Am. Math. Soc. 232 (1990), 251-268.

[26] J.F. Colombeau, New generalized functions and multiplication of distributions, Elsevier, Amsterdam, 2000.

[27] J.F. Colombeau, Elementary introduction to new generalized functions, Elsevier, Amsterdam, 2011

[28] E. Cordero, K. Gröchenig and L. Rodino, Localization operators and time-frequency analysis, in Harmonic, Wavelet and p-adic Analysis, Chuong et al. (editors), World Scientific, Singapore 2007, 83-109.

[29] E. Cordero, S. Pilipović, L. Rodino and N. Teofanov, Quasianalytic Gelfand-Shilov spaces with application to localization operators. Rocky Mountain J. Math. 404 (2010), 11231147.

[30] E. Cordero and F. Nicola, On the Schrödinger equation with potential in modulation spaces, preprint, arXiv:1405.3121 (2014).

[31] E. Cordero, F. Nicola and L. Rodino, Time-frequency analysis of Fourier integral operators, preprint, arXiv:0710.3652 (2007).

[32] E. Cordero, F. Nicola, L. Rodino, Boundedness of Fourier integral Operators on $\mathcal{F} L^{p}$ spaces, Trans. Amer. Math. Soc., 361 (2009), 6049-6071.

[33] E. Cordero, F. Nicola, L. Rodino, On the global boundedness of Fourier integral operators, Ann. Global Anal. Geom., 384 (2010), 373-398.

[34] E. Cordero, F. Nicola and L. Rodino, Propagation of the Gabor Wave Front Set for Schrödinger Equations with non-smooth potentials, preprint, arXiv:1309.0965 (2013).

[35] E. Cordero and L. Rodino, Wick calculus: a time-frequency approach, Osaka J. Math. 42 1 (2005), 43-63.

[36] H.O. Cordes, The Technique of Pseudodifferential Operators, Cambridge Univ. Press, 1995.

[37] A. Córdoba and C. Fefferman, Wave packets and Fourier integral operators, Communications in Partial Differential Equations 311 (1978), 979-1005.

[38] S. Coriasco, Fourier integral operators in SG classes I, Rend. Sem. Mat. Univ. Pol. Torino 574 (1999), 249-302.

[39] S. Coriasco, Fourier integral operators in SG classes II: Application to SG hyperbolic Cauchy problems, Ann. Univ. Ferrara, Sez. VII, Sc. Mat. 44 (1998), 81-122.

[40] S. Coriasco, K. Johansson, J. Toft, Local wave front sets of Banach and Fréchet types, and pseudodifferential operators, Monatshefte Math. 16934 (2013), 285-316, DOI 10.1007/s00605-012-0392-y

[41] S. Coriasco, K. Johansson, J. Toft, Global wave-front sets of Banach, Fréchet and modulation space types, and pseudo-differential operators, J. Differ. Equ. 2548 (2013), 32283258, DOI 10.1016/j.jde.2013.01.014.

[42] S. Coriasco, K. Johansson, J. Toft, Global wave-front sets of intersection and union type, Fourier Analysis, Trends in Mathematics 2014, Springer International Publishing, 2014, 91-106.

[43] S. Coriasco, K. Johansson and J. Toft, Global Wave Front Set of Modulation Space types and Fourier Integral Operators, preprint, arXiv:1307.6249 (2013).

[44] S. Coriasco and L. Maniccia, Wave front set at infinity and hyperbolic linear operators with multiple characteristics, Ann. Global Anal. and Geom., 24 (2003), 375-400. 
[45] S. Coriasco and P. Panarese, S. Coriasco, P. Panarese. Fourier Integral Operators in SG Classes: Classical Operators. In Partial Differential Equations and Spectral Theory, M. Demuth and B.-W. Schulze Editors (BirkhŠuser), Proceedings PDE 2000 Conference in Clausthal (Germany, 24-28/07/2000), Operator Theory, Advances and Applications 126 (2001), 81-91.

[46] S. Coriasco and P. Panarese, Fourier Integral Operators Defined by Classical Symbols with Exit Behaviour, Math. Nachr., 2421 (2002), 61-78.

[47] S. Coriasco and M. Ruzhansky, Global L $L^{p}$ continuity of Fourier integral operators, Trans. Amer. Math. Soc. 366 (2014), 2575-2596.

[48] S. Coriasco and J. Toft, Asymptotic expansions for Hörmander symbol classes in the calculus of pseudo-differential operators, J. Pseudo.-Differ. Oper. Appl. 5 (2014), 27-41.

[49] S. Coriasco and R. Schulz, The global wave front set of tempered oscillatory integrals with inhomogeneous phase functions, J. Fourier Anal. Appl. 195 (2013), 1093-1121, DOI 10.1007/s00041-013-9283-4.

[50] S. Coriasco and R. Schulz, SG-Lagrangian submanifolds and their parametrization, preprint, arXiv:1406.1888 (2014).

[51] M. deGosson, Symplectic Methods in Harmonic Analysis and in Mathematical Physics, Birkhäuser, Basel, 2011.

[52] J.-M. Delort, FBI transformation: second microlocalization and semilinear caustics, Vol. 1522, Springer-Verlag, Berlin, 1992.

[53] S. Doplicher, K. Fredenhagen and J. E. Roberts, The Quantum structure of space-time at the Planck scale and quantum fields, Commun. Math. Phys. 1721 (1995).

[54] M. Dubois-Violette, A. Kriegl, Y. Maeda and P.W. Michor, Smooth $C^{*}$-algebras, Prog. Theor. Phys. Suppl. 144 (2001), 54-78.

[55] J. J. Duistermaat, Fourier integral operators, Springer, Berlin, 1996.

[56] J. J. Duistermaat and L. Hörmander, Fourier integral operators II, Acta mathematica 1281 (1972), 183-269.

[57] Y. V. Egorov and B.-W. Schulze, Pseudo-Differential Operators, Singularities, Applications, Birkhäuser, Basel, 1997.

[58] H. Epstein and V. Glaser, The role of locality in perturbation theory, Ann. Inst. Henri Poincaré-Section A, 193 (1973), 211-295.

[59] H. Epstein and V. Glaser, Adiabatic limit in perturbation theory, Renormalization theory, Springer Netherlands, 1976, 193-254.

[60] H.G. Feichtinger, Modulation Spaces: Looking Back and Ahead, Sampl. Theory Signal Image Process. 52 (2006).

[61] G. B. Folland, Harmonic Analysis in Phase Space, Princeton University Press, 1989.

[62] G. B. Folland, Real Analysis: modern techniques and their applications, Wiley, New York, 1999.

[63] I.M. Gelfand and G.E. Shilov, Generalized functions II, Academic Press, New York, 1968.

[64] V. Guillemin and S. Sternberg, Geometric asymptotics, Amer. Math. Soc. Survey 14 (1977).

[65] P. Gérard, Moyennisation et régularité deux-microlocale, Ann. scient. Ec. Norm. Sup. 4éme série, 23 (1990), 89-121.

[66] J. M. Gracia-Bondía and J. C. Várilly, Algebras of distributions suitable for phasespace quantum mechanics I, Math. Phys. 294 (1988), 869-879.

[67] A. Grigis and J. Sjöstrand, Microlocal analysis for differential operators, Cambridge University Press, 1994.

[68] K. Gröchenig, Foundations of Time-Frequency Analysis, Birkhäuser, Boston, 2001. 
[69] K. Gröchenig and G. Zimmermann, Hardy's Theorem and the Short-time Fourier transform of Schwartz functions, J. London Math. Soc., 631 (2001), 205-214.

[70] A. Hassel and A. Vasy, The spectral projections and the resolvent for scattering metrics, J. Anal. Math., 791 (1999), 241-298.

[71] A. Hassel and J. Wunsch, The semiclassical resolvent and the propagator for non-trapping scattering metrics, Adv. Math. 2172 (2008), 586-682.

[72] D. Hilbert, Mathematische Probleme, Göttinger Nachrichten, 1900, pp. 253-297.

[73] E. Hille, A Class of Reciprocal Functions, Ann. Math. 274 (1926), 427-464.

[74] S. Hollands and R. Wald, Existence of Local Covariant Time Ordered Products of Quantum Fields in Curved Spacetime, Comm. Math. Phys. 2312 (2002), 309-345.

[75] S. Hollands and R. Wald, Axiomatic Quantum Field Theory in Curved Spacetime, Comm. Math. Phys. 2931 (2010), 85-125.

[76] A. Holst, J. Toft and P. Wahlberg, Weyl product algebras and modulation spaces, J. Funct. Ana. 251 (2007), 463-491.

[77] L. Hörmander, Linear differential operators, Actes Congr. Int. Math. Nice 1970, 1, 121133.

[78] L. Hörmander, Uniqueness theorems and wave front sets for solutions of linear differential equations with analytic coefficients, Comm. Pure Appl. Math. 245 (1971), 671-704.

[79] L. Hörmander, Fourier Integral operators I, Acta Math. 1271 (1971), 79-183.

[80] L. Hörmander, The Weyl calculus of pseudodifferential operators, Comm. Pure Appl. Math., 32 (1979), 360-444.

[81] L. Hörmander, The Analysis of Linear Partial Differential Operators, Vol. I, Springer, 1990.

[82] L. Hörmander, Quadratic hyperbolic operators, Microlocal Analysis and Applications, LNM vol. 1495, L. Cattabriga, L. Rodino (Eds.), 1991, 118-160.

[83] L. Hörmander, The Analysis of Linear Partial Differential Operators, Vol. III, Springer, 1994.

[84] L. Hörmander, The Analysis of Linear Partial Differential Operators, Vol. IV, Springer, 1994.

[85] K. Ito, Propagation of singularities for Schrödinger equations on the Euclidean space with a scattering metric, Comm. Partial Differential Equations 3112 (2006), 1735-1777.

[86] K. Ito, On the propagation of the homogeneous wavefront set for Schrodinger equations and on the equivalence of the homogeneous and the qsc wavefront sets, Research Institute for Mathematical Sciences (RIMS) Kokyu, 2006, 182-198.

[87] A. Kaminski and S. Mincheva-Kaminska Compatibility Conditions and the Convolution of Functions and Generalized Functions, J. Funct. Space. Appl. (2013).

[88] A. Kaneko, On the global existence of real analytic solutions of linear partial differential equations on unbounded domain, J. Fac. Sci. Univ. Tokyo Sec. IA 32 (1985), 319-372.

[89] T. Kawai, On the theory of Fourier hyperfunctions and its applications to linear partial differential equations with constant coefficients, J. Fac. Sci. Univ. Tokyo Sec. IA 17 (1970), $467-517$.

[90] H. Komatsu, Ultradistributions I: Structure theorems and a characterization, J. Fac. Sci. Univ. Tokyo Sec. IA 20 (1973), 25-105.

[91] H. Komatsu, Ultradistributions II: The kernel theorem and ultradistributions with support in a submanifold, J. Fac. Sci. Univ. Tokyo Sec. IA 24 (1977), 607-628.

[92] H. Komatsu, Ultradistributions, III. Vector valued ultradistributions and the theory of kernels, J. Fac. Sci. Univ. Tokyo Sec. IA 29 (1982), 653-717.

[93] G. Lebeau, Equations des ondes semi-linéaires II. Contrôle des singularités et caustiques non-linéaires, Invent. Math. 952 (1989), 277-323. 
[94] N. Lerner, Metrics on the Phase Space and Non-Selfadjoint Pseudo-Differential Operators, Birkhäuser, Basel, 2010.

[95] N. Lerner, A tribute to Lars Hörmander, Eur. Math. Soc. Newsl. 88 (2013), 24-34.

[96] S. Łojasiewicz, Sur la valeur et la limite d'une distribution en un point, Studia Mathematica 161 (1957), 1-36.

[97] J. M. Maillard, On the twisted convolution product and the Weyl transformation of tempered distributions, J. Geom. Phys. 32 (1986), 231-261.

[98] J. Margalef-Roig and E. Outerelo Dominguez, Differential topology, Elsevier, 1992.

[99] A. Martinez, An Introduction to Semiclassical and Microlocal Analysis, Universitext, Springer, New York, 2002.

[100] A. Martinez, S. Nakamura and V. Sordoni, Analytic smoothing effect for the Schrödinger equation with long-range perturbation, Comm. Pure Appl. Math. 59 (2006), 1330-1351.

[101] A. Martinez, S. Nakamura and V. Sordoni, Analytic wave front set for solutions to Schrödinger equations, Adv. Math. 2224 (2009), 1277-1307.

[102] R. Melrose, Spectral and scattering theory for the Laplacian on asymptotically Euclidian spaces. In "Spectral and scattering theory", Sanda 1992. Lecture Notes in Pure and Appl. Math., 161, Dekker, New York, 85-130 (1994).

[103] R. Melrose, Geometric scattering theory, Stanford Lectures, Cambridge Univ. Press, Cambridge, 1995.

[104] R. Melrose, Fibrations, compactifications and algebras of pseudodifferential operators, Partial Differential Equations and Mathematical Physics, Birkhäuser Boston, 1996, 246-261.

[105] R. Melrose, Differential analysis on manifolds with corners, unfinished, available online at http://www-math.mit.edu/ rbm/book.html, last downloaded 08/08/2014.

[106] R. Melrose, Lecture Notes for Graduate Analysis, 2004, available online at http://wwwmath.mit.edu/ rbm/18.155-F04-notes/Lecture-notes.pdf, last downloaded 08/08/2014.

[107] R. Melrose and M. Zworski, Scattering metrics and geodesic flow at infinity, Inventiones Mathematicae 124 1-3 (1996), 389-436.

[108] Y. Meyer, Wavelets, Vibrations and Scalings, CRM monograph series, AMS, vol. 9 (1997).

[109] R. Mizuhara, Microlocal smoothing effect for the Schrödinger equation in a Gevrey class, J. Math. Pures Appl. 912 (2009), 115-136.

[110] S. Nagamachi and T. Nishimura, Edge of the wedge theorem for Fourier hyperfunctions, Funkcial. Ekvac. 36 (1993), 499-517.

[111] S. Nakamura, Propagation of the homogeneous wave front set for Schrödinger equations, Duke Math. J. 1262 (2005), 349-367.

[112] V. D. Nguyen, Renormalization of quantum field theory on curved space-times, a causal approach, Diss. Université Paris-Diderot-Paris VII, 2013.

[113] F. Nicola and L. Rodino, Global Pseudo-differential Calculus on Euclidean Spaces, Birkhäuser, Basel, 2010.

[114] C. Parenti, Operatori pseudo-differentiali in $\mathbb{R}^{n}$ e applicazioni, Annali Mat. Pura Appl., 93 (1972), 359-389.

[115] S. Pilipovic and J. Toft, On the quasianalytic wave-front set, preprint, arXiv:1210.7741v1 (2012).

[116] S. Pilipovic and J. Toft, Wave-front sets related to quasi-analytic Gevrey sequences, preprint, arXiv:1210.7741v2 (2014).

[117] M. Reed and B. Simon, Methods of modern mathematical physics I, Acad. Press, New York, 1972.

[118] M. Reed and B. Simon, Methods of modern mathematical physics II, Acad. Press, New York, 1975.

[119] L. Rodino, Polysingular integral operators, Ann. Mat. Pura Appl. 124 (1980), 59-106. 
[120] L. Rodino, Linear partial differential operators in Gevrey spaces, World Scientific, Singapore, 1993.

[121] L. Rodino and P. Wahlberg, The Gabor wave front set, Monatsh. Math. 173 (2014), 625-655, DOI 10.1007/s00605-013-0592-0.

[122] W. Rudin, Functional analysis, 2nd ed., International Series in Pure and Applied Mathematics, McGraw-Hill, New York, 1991.

[123] Michael Ruzhansky and Mitsuru Sugimoto, Global boundedness theorems for Fourier integral operators associated with canonical transformations, Comm. Partial Differential Equations 314 (2006), 547-569.

[124] M. Sato, Theory of Hyperfunctions, I, J. Fac. Sci. Univ. Tokyo Sect. IA 81 (1959), $139-193$.

[125] M. Sato, Theory of Hyperfunctions, II, J. Fac. Sci. Univ. Tokyo Sect. IA 82 (1960), $387-437$.

[126] M. Sato, T. Kawai and M. Kashiwara, Hyperfunctions and pseudodifferential equations, Lecture Notes in Math. 287 (1973), 265-529.

[127] G. Scharf, Finite quantum electrodynamics, Springer, Berlin, 1989.

[128] E. Schrohe, Spaces of weighted symbols and weighted Sobolev spaces on manifolds, Springer Berlin Heidelberg, 1987, 360-377.

[129] R. Schulz, Produkte temperierter Distributionen (Master's thesis), Universität Göttingen, 2011.

[130] R. Schulz and P. Wahlberg, The equality of the homogeneous and the Gabor wave front set, preprint, arXiv:1304.7608 (2013).

[131] B.-W. Schulze, Boundary value problems and singular pseudo-differential operators, J. Wiley, Chichester, 1998.

[132] L. Schwartz, Théorie des distributions I-II, Hermann, Paris, 1950-1951.

[133] L. Schwartz, Sur l'impossibilité de la multiplication des distributions, CR Acad. Sci., Paris, 239 (1954), 847-848.

[134] R. T. Seeley, Extension of $\mathscr{C}^{\infty}$ functions defined in a half space Proc. Amer. Math. Soc. $\mathbf{1 5}(1964), 625-626$.

[135] M. Shubin, Pseudodifferential operators and the spectral theory. Springer Series in Soviet Mathematics. Springer-Verlag, Berlin, 1987.

[136] J. Sjöstrand, Singularités analytiques microlocales. Astérisque 95(1982), 1-166.

[137] M.A. Soloviev, Star product algebras of test functions, Theor. Math. Phys. 153 (2007), 1351-1363

[138] M.A. Soloviev, Moyal multiplier algebras of the test function spaces of type S, J. Math. Phys. 526 (2011), 063502.

[139] M.A. Soloviev, Generalized Weyl correspondence and Moyal multiplier algebras, Theo. and Math. Phys. 1731 (2012), 1359-1376.

[140] O. Steinmann, Perturbation Expansions in Axiomatic Field Theory, Lecture Notes in Physics 11, Springer, Berlin, 1971.

[141] N. Teofanov, Ultradistributions and time-frequency analysis. Pseudo-differential operators and related topics, Oper. Theory Adv. Appl., 164, Birkhäuser, Basel, 2006, 173-192.

[142] J. Toft, The Bargmann transform on modulation and Gelfand-Shilov spaces, with applications to Toeplitz and pseudo-differential operators, J. Pseudo-Differ. Oper. Appl., 32 (2012), 145-227.

[143] J. Toft, The range of localization operators and lifting theorems for modulation and Bargmann-Fock spaces, Trans. Amer. Math. Soc. 3658 (2013), 4475-4496.

[144] J. Toft, Multiplication properties in pseudo-differential calculus with small regularity on the symbols, J. Pseudo-Differ. Oper. Appl. 1 (2010), 101-138. 
[145] F. Treves, Introduction to pseudodifferential and Fourier integral operators, Vol. 1-2. The University Series in Mathematics. Plenum Press, New York-London, 1980.

[146] H. Triebel, Theory of function spaces III, Birkhäuser, Basel, 2006.

[147] S. Wakabayashi, Classical Microlocal Analysis in the Space of Hyperfunctions, Springer, Berlin, 2000.

[148] A. Weinstein, The order and symbol of a distribution, Trans. Am. Math. Soc. 241 (1978), $1-54$.

[149] A. Weinstein, A symbol class for some Schrödinger equations on $\mathbb{R}^{n}$, Amer. J. Math. 107 (1985), 1-21.

[150] H. Whitney, Analytic extensions of differentiable functions defined in closed sets, Trans. Amer. Math. Soc. 36 (1934), 63-89.

[151] I. Witt, A Calculus for Classical Pseudo-Differential Operators with Non-Smooth Symbols, Math. Nachr. 194 (1998), 239-284.

[152] M. Wrochna, Singularities of two-point functions in Quantum Field Theory, Diss. Niedersächsische Staats-und Universitätsbibliothek Göttingen, 2013.

[153] J. Wunsch, Propagation of singularities and growth for Schrödinger operators, Duke Math. J. 98 (1999), 137-186.

[154] J. Wunsch, The trace of the generalized harmonic oscillator, Ann. Inst. Fourier 491 (1999), 351-373.

[155] J. Wunsch and M. Zworski, The FBI transform on compact $\mathscr{C}^{\infty}$-manifolds, Trans. Amer. Math. Soc. 3533 (2001), 1151-1167.

[156] J. Zahn, The wave front set of oscillatory integrals with inhomogeneous phase function, J. Pseudo-Differ. Oper. Appl. 2 (2011), 101-113.

[157] M. Zworski, Semiclassical Analysis, Graduate Studies in Mathematics 138, AMS, 2012. 
René Marcel Schulz

Mathematisches Institut

Georg-August Universität Göttingen

Bunsenstraße 3-5

Born 23 November 1987,

D-37073 Göttingen

rschulz@uni-math.gwdg. de

\section{Curriculum Vitae}

\section{Education}

$10 / 2011-09 / 2014$

Georg-August Universität Göttingen

$\begin{array}{ll}10 / 2011-09 / 2014 & \begin{array}{l}\text { PhD studies in mathematics } \\ \text { In the Research Training Group 1 } \\ \text { Supervised by Prof. Dr. Dorothea } \\ \text { Thesis title: "Microlocal Analysis }\end{array} \\ 04 / 2010-11 / 2011 & \begin{array}{l}\text { Master of Science in mathematics } \\ \text { Thesis title: "Produkte temperier }\end{array} \\ 12 / 2006-04 / 2010 & \text { Bachelor of Science in mathematic } \\ 10 / 2006-02 / 2010 & \text { Bachelor of Science in physics } \\ & \begin{array}{l}\text { Other } \\ \text { Università degli Studi di Torino }\end{array} \\ 09 / 2013 ; 04 / 2013 ; & \begin{array}{l}\text { Research visits } \\ 07 / 2012-10 / 2012\end{array} \\ 07 / 2009-12 / 2009 & \begin{array}{l}\text { University of Technology Sydney } \\ \text { Semester abroad }\end{array} \\ 02 / 2000-06 / 2006 & \begin{array}{l}\text { Otto-Hahn-Gymnasium Gifhorn } \\ \text { Abitur }\end{array}\end{array}$

\section{Working experience}

$05 / 2013$ - 10/2013;

$05 / 2011$ - 10/2011;

$04 / 2010-12 / 2010$

07/2006 - 08/2006

\section{Other}

\section{Academia}

Georg-August-Universität Göttingen

Graduate assistant

Physikalisch Technische Bundesanstalt, Braunschweig Internship 